\title{
Measurement of Electrical Current Density Distribution within the tissues of the head by Magnetic Resonance Imaging
}

\author{
Humberto Remigio Gamba

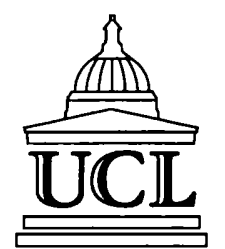 \\ Department of Medical Physics \& Bioengineering \\ University College London \\ Submitted for \\ The Degree of Doctor of Philosophy \\ University of London
}

May 1996 
ProQuest Number: 10045488

All rights reserved

INFORMATION TO ALL USERS

The quality of this reproduction is dependent upon the quality of the copy submitted.

In the unlikely event that the author did not send a complete manuscript and there are missing pages, these will be noted. Also, if material had to be removed, a note will indicate the deletion.

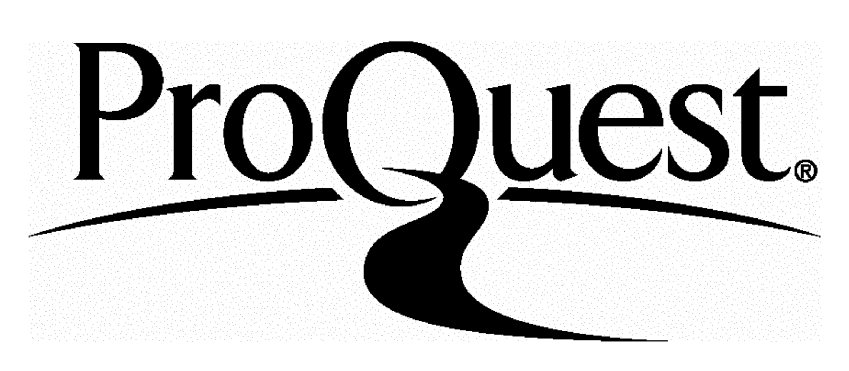

ProQuest 10045488

Published by ProQuest LLC(2016). Copyright of the Dissertation is held by the Author.

All rights reserved.

This work is protected against unauthorized copying under Title 17, United States Code. Microform Edition $\odot$ ProQuest LLC.

\author{
ProQuest LLC \\ 789 East Eisenhower Parkway \\ P.O. Box 1346 \\ Ann Arbor, MI 48106-1346
}




\section{Abstract}

In vivo and in vitro images of the distribution of electrical current inside the head of newborn piglets that arise from electrodes placed on the top of the head and in the root of the mouth are presented in this thesis. The images are obtained from the magnetic field induced by the electrical current propagating inside the head which is measured using nuclear magnetic resonance (NMR) phase images. From the phase images the electrical current images are then calculated using Ampere's law.

Following a review of the NMR principles, the technique of measuring electrical current by NMR is described in detail. The implementation of the technique was then validated with both numerical simulations and experiments with a cylindrical and a cubical phantom. The sources of noise in the NMR data are also reviewed and their effect in the calculated current images is analysed both theoretically and experimentally using the phantoms.

From the current images, preferential paths used by the electrical current in passing from the electrode under the neck to the electrode on the top of the head could be identified.

The total current per region in the head is determined by segmenting the NMR intensity image and integrating the current through each region. Remarkable differences in the current distribution between the in vivo and post mortem experiment were found. The piglet head inclination during the in vivo experiment was also found to affect the current orientation. It was thought that this effect might be related to a shift in blood volume or flow when the head is inclined, and some evidence for such a change in blood volume, measured using near infrared spectroscopy is presented. 


\section{ERRATA}

page 54 - The fifth line should read : " component (sine or cosine) "

page 70 - The first sentence of the first paragraph should start : The algorithm suggested by Redefield and Kunz (1975) ..."

page 105 - The second sentence of the fourth paragraph should read : "The cylinder bases are two $100 \times 100 \mathrm{~mm}$ squares..."

page 113 - Figures 4.10 (a) and (c) the right graybar scale should be : " $-4.5 \mu A+4.5 \mu A$ "

page 125 - The first sentence of second paragraph should read : " For the current image calculated from the field measured in the NMR experiment, the integrated value of the inner cylinder current is $9.7 \mathrm{~mA}$. "

page 156 - The fourth line from the top should be : " Gudbjartsson (1995) "

page 177 - The third sentence of the first paragraph should read : " The outer contour encompassed the whole head and was drawn using the current images in figures 7.16 to try and avoid the edge artifacts. "

page 187 - The first sentence of the last paragraph should start : " The spin-echo sequence used ..."

page 231 - $\quad$ reference [84] : " Webb, S. (1995), The Physics of Medical Imaging, Institute of Physics Publishing. "

page 226 - include reference : " Cole, K. S. (1968) Membranes, ions and impulses: a chapter of classical biophysics", Cambridge Press. " 


\section{Contents}

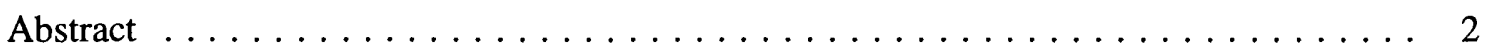

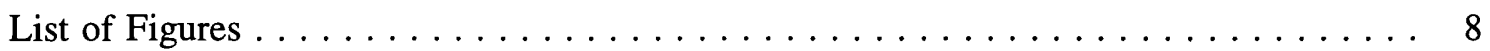

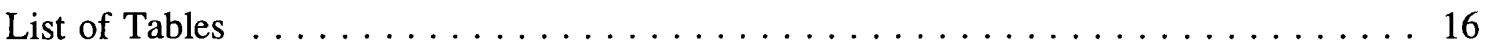

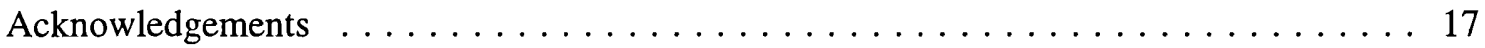

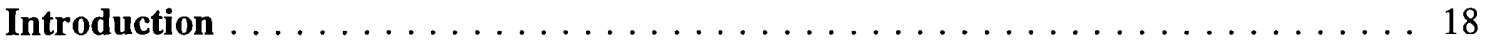

\section{Chapter 1}

1. Electrical Impedance Tomography $\ldots \ldots \ldots \ldots \ldots \ldots \ldots \ldots \ldots \ldots \ldots \ldots \ldots$

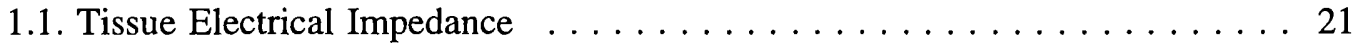

1.1.1. Electric circuit model of tissue $\ldots \ldots \ldots \ldots \ldots \ldots \ldots \ldots 21$

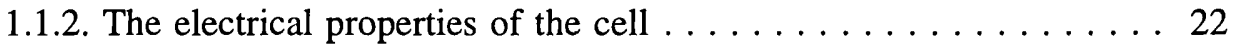

1.1.3. Measurement of tissue electrical impedance . . . . . . . . 23

- two-electrode technique ................ 23

-four-electrode technique ............... 23

- Current density measurement . . . . . . . . . . . . . 24

1.1.4. Values of conductivity of tissue at low frequencies $\ldots \ldots \ldots 26$

1.2. Electrical Impedance Tomography . . . . . . . . . . . . . . . . 28

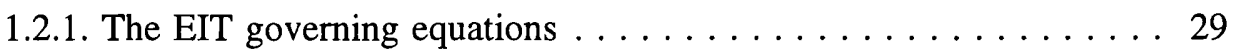

1.2.3. Applied Potential Tomography . . . . . . . . . . . . 31

1.2.4. The data acquisition system and image resolution $\ldots \ldots \ldots 32$

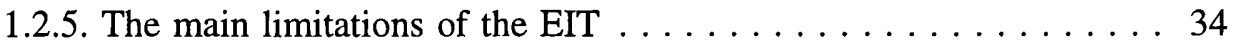

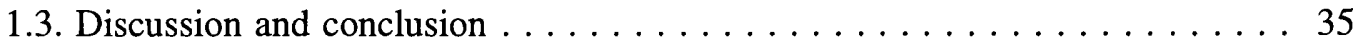

\section{Chapter 2}

2. Nuclear Magnetic Resonance . . . . . . . . . . . . . . . . . . . 37

2.1. Principles of Nuclear Magnetic Resonance . . . . . . . . . . . . . . 37

2.1.1. Angular Momentum and Magnetic Moment . . . . . . . . . 37

2.1.2. Nuclear precession in a static magnetic field . . . . . . . . 41

2.1.3. Radiofrequency absorption and Macroscopic Magnetisation . . . . . 42

2.1.4. The rotating frame concept $\ldots \ldots \ldots \ldots \ldots \ldots \ldots$ 
2.1.5. The RF Field $B_{1} \ldots \ldots \ldots \ldots \ldots \ldots \ldots$

2.1.6. The Relaxation Phenomena and Bloch's equations ........ . 49

2.1.7. The free induction decay and spin echo signals $\ldots \ldots \ldots \ldots 52$

2.2. Imaging principles $\ldots \ldots \ldots \ldots \ldots \ldots \ldots \ldots \ldots \ldots \ldots \ldots \ldots \ldots \ldots$

2.2.1. The slice selection $\ldots \ldots \ldots \ldots \ldots \ldots \ldots \ldots \ldots \ldots \ldots \ldots \ldots$

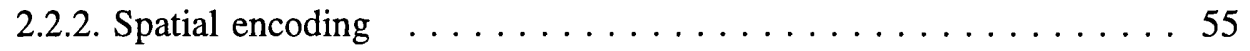

2.2.3. The spin-echo imaging sequence $\ldots \ldots \ldots \ldots \ldots \ldots \ldots$

2.3. NMR image reconstruction $\ldots \ldots \ldots \ldots \ldots \ldots \ldots \ldots \ldots \ldots \ldots \ldots$

2.3.1. NMR signal demodulation $\ldots \ldots \ldots \ldots \ldots \ldots \ldots \ldots \ldots 6$

2.3.2. Signal digitisation $\ldots \ldots \ldots \ldots \ldots \ldots \ldots \ldots \ldots \ldots \ldots \ldots \ldots$

2.3.3. The two-dimensional discrete Fourier transform . . . . . . . 63

2.3.3.1. The Redfield and Kunz treatment . . . . . . . . . 67

2.3.4. The theory of NMR phase-correction $\ldots \ldots \ldots \ldots \ldots$

2.3.5. The Fourier transform and the phase-correction algorithms . . . . 73

2.4. The Bruker spin-echo sequence $\ldots \ldots \ldots \ldots \ldots \ldots \ldots \ldots \ldots \ldots \ldots \ldots \ldots$

2.5. Conclusion . . . . . . . . . . . . . . . . . . . . . . . . . 77

\section{Chapter 3}

3. Imaging of Electrical Current Distribution $\ldots \ldots \ldots \ldots \ldots \ldots \ldots \ldots \ldots \ldots$

3.1. The NMR experiment with applied electrical current pulses $\ldots \ldots \ldots .81$

3.2. Determination of the phase shift caused by the current $\ldots \ldots \ldots \ldots 2$

3.3. Phase "unwrapping" . . . . . . . . . . . . . . . . . . . 86

3.3.1. Phase unwrapping: Basic principles $\ldots \ldots \ldots \ldots . \ldots 87$

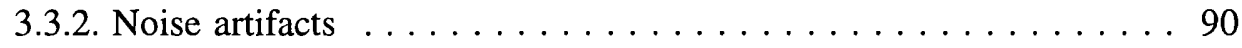

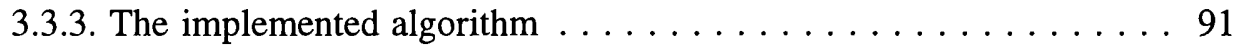

3.4. Electrical Current Image Distribution Calculation . . . . . . . . . . 95

3.5. Image registration $\ldots \ldots \ldots \ldots \ldots \ldots \ldots \ldots \ldots \ldots \ldots \ldots \ldots \ldots \ldots$

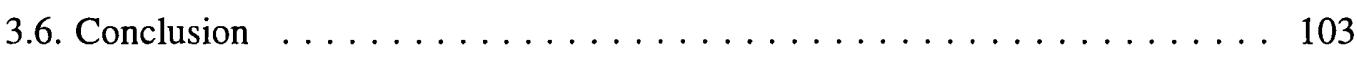

\section{Chapter 4}

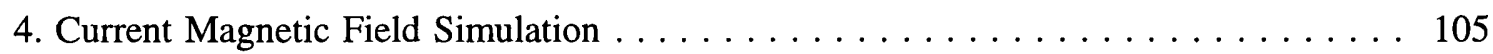

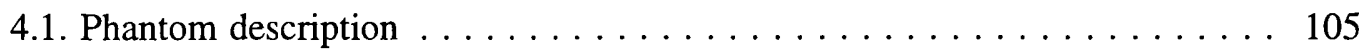

4.2. The magnetic field equations $\ldots \ldots \ldots \ldots \ldots \ldots \ldots \ldots \ldots \ldots \ldots \ldots$

4.3. Simulation and experiment results $\ldots \ldots \ldots \ldots \ldots \ldots \ldots \ldots$

4.4. Electrical current images . . . . . . . . . . . . . . . . . . 113

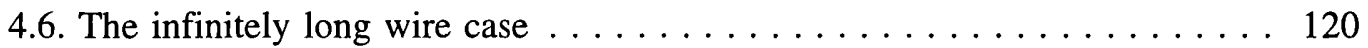




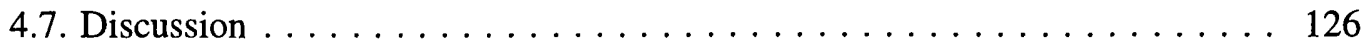

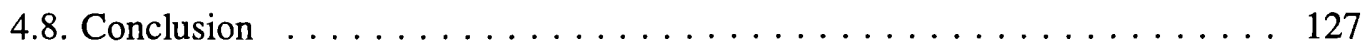

\section{Chapter 5}

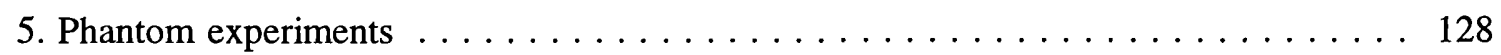

5.1. Cubic phantom experiment $\ldots \ldots \ldots \ldots \ldots \ldots \ldots \ldots \ldots \ldots \ldots \ldots \ldots$

5.1.1. Phantom description $\ldots \ldots \ldots \ldots \ldots \ldots \ldots \ldots \ldots \ldots \ldots$

5.1.2. NMR image parameters and electrical current . . . . . . . 130

5.1.3. Results . . . . . . . . . . . . . . . 130

5.1.3.1. Electrical current magnetic field $\ldots \ldots \ldots \ldots \ldots . \ldots 131$

5.1.3.2. Current images ................ 133

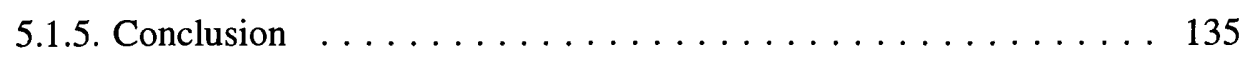

5.2. Experiment performed with a EIT tank phantom . . . . . . . 135

5.2.1. Phantom description $\ldots \ldots \ldots \ldots \ldots \ldots \ldots \ldots$

5.2.3. Results . . . . . . . . . . . . . . . . . . 137

5.2.3.1. Current density in the EIT tank with adjacent electrode drive . . . . . . . . . . . . . . . 137

5.2.3.2. Current density in the EIT tank with opposite electrode drive ..................... 138

5.2.4. Discussion and conclusion . . . . . . . . . . . . . 142

\section{Chapter 6}

6. Electrical current noise analyses $\ldots \ldots \ldots \ldots \ldots \ldots \ldots \ldots \ldots \ldots \ldots$

6.1. Noise sources and SNR in NMR . . . . . . . . . . 143

6.2. Error propagation in NMR phase images $\ldots \ldots \ldots \ldots \ldots \ldots \ldots$

6.2.1. Phase error propagation experiments . . . . . . . . . 149

6.3. Error propagation in the current image $\ldots \ldots \ldots \ldots \ldots \ldots \ldots$

6.3.1. Current image error propagation experiment $\ldots \ldots \ldots \ldots$

6.4. Mis-registration error propagation $\ldots \ldots \ldots \ldots \ldots \ldots \ldots \ldots \ldots$

6.5. The difference when SNR is lower than two . . . . . . . . 155

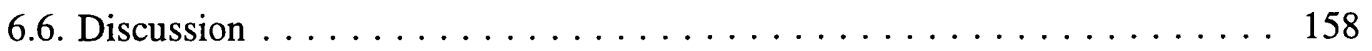

6.7. Conclusion ... . . . . . . . . . . . . . . . . . . 159

\section{Chapter 7}

7. In vitro experiments with new born piglet $\ldots \ldots \ldots \ldots \ldots \ldots \ldots \ldots \ldots$

7.1. In vitro experiments with isolated head $\ldots \ldots \ldots \ldots \ldots \ldots \ldots$ 
7.1.1. Methodology . . . . . . . . . . . . . . . . . . 160

7.1.1.1. The piglet's head holder and image markers . . . . 161

7.1.1.2. The electrodes and the electrical current pulses . . . 162

7.1.1.3. The NMR image parameters $\ldots \ldots \ldots \ldots \ldots \ldots$

7.1.2. Results for the first experiment $\ldots \ldots \ldots \ldots \ldots \ldots \ldots$

7.1.2.1. Electrical current magnetic field . . . . . . . . 164

7.1.2.2. Electrical current images ............. 165

7.1.2.3. Current per region in the head $\ldots \ldots \ldots \ldots \ldots .168$

7.1.3. Results of the second experiment . . . . . . . . . . . . 169

7.2. In vitro experiments with an intact piglet $\ldots \ldots \ldots \ldots \ldots \ldots \ldots \ldots$

7.2.1. Methodology . . . . . . . . . . . . . . . 172

7.2.1.1. The mechanism to rotate the frame . . . . . . 172

7.2.1.2. The electrodes and the electrical current pulses .... 173

7.2.1.3. The NMR image parameters . . . . . . . 173

7.2.1.4. The field projection algorithm to determine $B_{y} \ldots \ldots 174$

7.2.2. Results . . . . . . . . . . . . . . . . . . . . . . . . . . . . 174

7.2.2.1. Current magnetic field images $\ldots \ldots \ldots \ldots \ldots \ldots$

7.2.2.3. Current per region in the head . . . . . . . . . 177

7.3. Noise analyses in the in vitro current images . . . . . . . . . . . 177

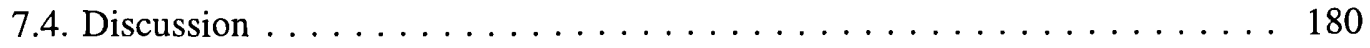

7.5. Conclusion $\ldots \ldots \ldots \ldots \ldots \ldots \ldots \ldots \ldots \ldots \ldots \ldots \ldots \ldots$

\section{Chapter 8}

8. In vivo experiment with a newborn piglet $\ldots \ldots \ldots \ldots \ldots \ldots \ldots \ldots \ldots$

8.1. Animal preparation . . . . . . . . . . . . . . . 184

8.2. The electrodes and the electrical current $\ldots \ldots \ldots \ldots \ldots \ldots \ldots$

8.4. The NMR image parameters $\ldots \ldots \ldots \ldots \ldots \ldots \ldots \ldots \ldots \ldots$

8.5. Results . . . . . . . . . . . . . . . . . . . 188

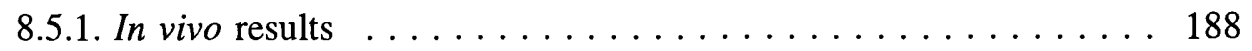

8.5.3. Electrical current magnetic field . . . . . . . . . . . . 190

8.5.4. Electrical current images . . . . . . . . . . . . . . 191

8.5.5. Electrical current per region in the head . . . . . . . 192

8.6. Results of the experiment performed after death $\ldots \ldots \ldots \ldots$

8.6.1. Electrical current magnetic field . . . . . . . . . . . . . . 194

8.6.2. Electrical current images . . . . . . . . . . . . . . 195

8.6.3. Electrical current per region in the head $\ldots \ldots \ldots \ldots \ldots$ 
8.7. Noise analyses $\ldots \ldots \ldots \ldots \ldots \ldots \ldots \ldots \ldots \ldots \ldots \ldots \ldots$

8.8. Discussion . . . . . . . . . . . . . . . . . . . . . 197

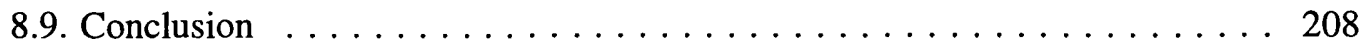

\section{Chapter 9}

9. Conclusions and future work . . . . . . . . . . . . . . . . . . 209

9.1. Results of the current measurements . . . . . . . . . . . . . . 209

9.2. The NMR technique implemented to measure electrical current . . . . . 211

9.3. Proposal for a new NMR technique to measure electrical current . . . . . 213

9.4. An additional study with the EIT tank . . . . . . . . . . . 214

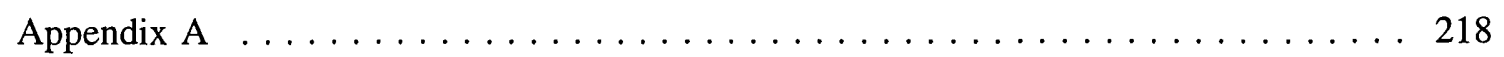

Appendix B . . . . . . . . . . . . . . . . . . . 219

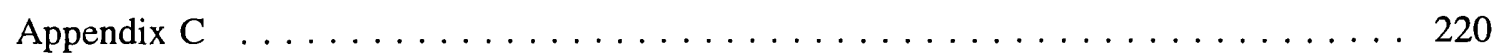

C. Programmable Current Source and NMR Trigger Control . . . . . . . . . . . 220

C.1. The Current Source Unit $\ldots \ldots \ldots \ldots \ldots \ldots \ldots \ldots \ldots \ldots \ldots \ldots$

C.2. Current Pulse and NMR Trigger Control. . . . . . . . . . . 222

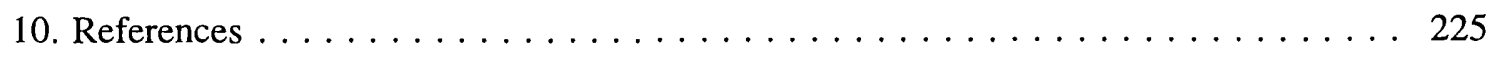




\section{List of Figures}

Figure 1.1 - Electrical model of the cell membrane (from Webster, 1990) . . . . . . . 21

Figure 1.2 - (a) series and (b) parallel representations of the tissue complex

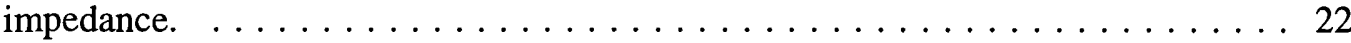

Figure 1.3 - Sketch showing errors which can arise due to electrode impedance $Z$ in a four-electrode system (from Barber \& Brown, 1984). . . . . . . . . . . . 25

Figure 1.4 - (a) Current spread in a conductor, and (b) Current spread with a guard electrode maintained at the same potential as the measuring electrode $M$ (from Geddes \& Baker 1989). . . . . . . . . . . . . . . . . . . . . . . . . 26

Figure 1.5- At high frequencies the current travels in the straight lines and at low frequencies the current travels longer paths through only the extracellular fluid (from Webster, 1990). . . . . . . . . . . . . . . . . . . . . . . . . . . 26

Figure 1.6 - An idealised plot of the frequency variation of the relative permittivity for a typical biological tissue (from Pethig, 1987) . . . . . . . . . . . . . . . 27

Figure 1.7 - The backprojection image reconstruction $\ldots \ldots \ldots \ldots \ldots \ldots \ldots$

Figure 1.8 - The isopotentials lines for different current patterns in a uniform

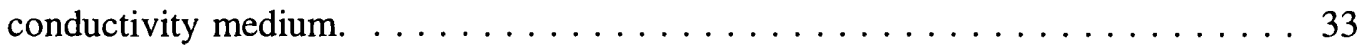

Figure 2.1- Classical model for the estimation of the angular momentum of a proton . . 38

Figure 2.2- The orbital angular momentum $\mathbf{L}$ and the orbital magnetic moment $\mu_{1}$ of an electron (from Eisberg, 1985). . . . . . . . . . . . . . . . . . . . . . . 39

Figure 2.3- Representing the angular momentum vector for the possible states $1=2$. In each state the vector is equally likely to be found anywhere on a cone

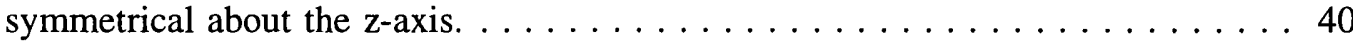

Figure 2.4 - Magnetic dipole representing the magnetic moment of the positive spinning charge in Figure 2.1. The magnetic field produces a torque about $o . \ldots \ldots \ldots 41$

Figure 2.5- A torque $\tau$ arises as the atom's magnetic dipole momentum $\mu_{1}$ interacts with the applied field $\mathbf{B}_{\mathbf{0}}$ (from Eisberg, 1985) . . . . . . . . . . . . . . . 42

Figure 2.6- The energy-level diagram for a spin $1 / 2$ system. Transitions between the two states can be induced by electromagnetic energy of frequency $\omega=(\gamma / 2 \pi) \mathrm{B}_{0} \ldots \ldots 43$

Figure 2.7 - The distribution of individual nuclear magnetic moments in the rotating frame for a hydrogen atom, spin $1 / 2 . \ldots \ldots \ldots \ldots \ldots \ldots \ldots \ldots$

Figure 2.8- (a) the magnetization $\mathbf{M}$ precessing around $\mathbf{B}_{\mathbf{0}}$ in the laboratory frame, (b) the magnetisation is static in the rotating frame since $\mathbf{B}_{\text {eff }}=0 \ldots \ldots \ldots 4$

Figure 2.9- (a) The rotating magnetic fields in the laboratory frame. (b) The rotation of $\mathbf{M}_{0}$ about $\mathbf{B}_{1}$ in the rotating frame. 
Figure 2.10 - The net magnetisation rotation. (a) a $+90^{\circ} \mathrm{B}_{1} \mathrm{RF}$ (b) $-90^{\circ} \mathrm{RF}$ pulse. . . . 48

Figure 2.11- Spin-lattice relaxation time $\mathrm{T}_{1}$ viewed from the rotating frame. After the $90^{\circ}$ pulse (a), the net magnetisation gradually returns to the original distribution

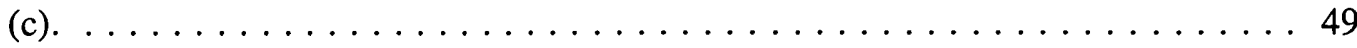

Figure 2.12- Spin-spin relaxation time $\mathrm{T}_{2}$ viewed from the rotating frame. $\ldots \ldots \ldots 50$

Figure $2.13-$ The $180^{\circ}$ refocusing pulse $\ldots \ldots \ldots \ldots \ldots \ldots \ldots \ldots \ldots \ldots \ldots \ldots \ldots \ldots \ldots$

Figure 2.14- Signal evolution $\mathbf{M}_{\mathrm{xy}} \mid$ in the spin echo-time sequence pulse. Upper: The $90^{\circ}$ and $180^{\circ} \mathrm{RF}$ pulses. Lower: The signal evolution. . . . . . . . . 53

Figure 2.15 - Illustration of the process to selected a tomographic plane. . . . . . . . 54

Figure 2.16-(a) Spin density of the slice selected in figure 2.15.(b) The resulting projection of solving the integral of equation (2.47) into the $y$-direction. . . . . 55

Figure 2.17- The phase encoding principle. (a) The frequency encoding; (b) The phase encoding followed by the frequency encoding (from Morris, 1986). . . . . . . 57

Figure 2.18 - The phase encoding modulation on the FID collection. . . . . . . 58

Figure $2.19-$ The NMR spin-echo pulses sequence $\ldots \ldots \ldots \ldots \ldots \ldots \ldots \ldots$

Figure 2.20 - Effects of the refocusing pulse in the net magnetisation. . . . . . . . 60

Figure 2.21- The quadrature detector to demodulate the NMR signal and the time sharing $\mathrm{ADC}$ converter system. $\ldots \ldots \ldots \ldots \ldots \ldots \ldots \ldots \ldots \ldots \ldots \ldots \ldots \ldots$

Figure 2.22 - (a) Real and (b) Imaginary time domain components. Both were simulated according to equation (2.65) to simulate the spectrum of $S_{n} \ldots \ldots 65$

Figure 2.23 - (a) Real and (b) Imaginary spectrum of the DFT of the time signal shown

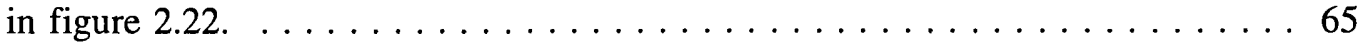

Figure 2.24 - Real (a) and Imaginary (b) time domain spin-echo signals of the phantom experiment. Echo time was $120 \mathrm{~ms}$, Repetition time $700 \mathrm{~ms}$ and slice thickness $5 \mathrm{~mm}$. (c) image profile of figure (b). . . . . . . . . . . . 66

Figure 2.25 - Images from the (a) Real and (b) Imaginary parts of the spectrum of the signal shown in figure $2.24 \ldots \ldots \ldots \ldots \ldots \ldots \ldots \ldots \ldots \ldots \ldots \ldots \ldots \ldots \ldots$

Figure 2.26 - (a) Phase and (b) Magnitude images of the signal shown in figure 2.24 . . 67

Figure 2.27- Real and imaginary spectrum, (a) and (b) respectively, of the DFT of the time domain signal shown in figure 2.22 after the Redfield and Kunz treatment $\ldots \ldots \ldots \ldots \ldots \ldots \ldots \ldots$. . . . . . . . . . . . . . . . . . 68

Figure 2.28 - Real (a) and Imaginary (b) images of the time domain signal shown in figure 2.24. The centre lines indicate the profile position shown in figure

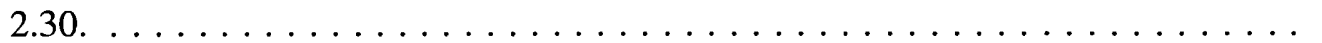

Figure 2.29 - Phase (a) and magnitude (b) images of the time signal shown in figure 
Figurer 2.30 - Horizontal centre profile of the real and imaginary images shown in figure $2.28 . \ldots \ldots \ldots \ldots \ldots \ldots \ldots \ldots \ldots \ldots \ldots \ldots \ldots \ldots \ldots \ldots \ldots \ldots \ldots \ldots \ldots \ldots$

Figure 2.31 - a) Real and b) Imaginary images after the phase correction. . . . . . . . 72

Figure 2.32 - a) Phase and b) magnitude images from the real and imaginary images shown in figure $2.31 . \ldots \ldots \ldots \ldots \ldots \ldots \ldots \ldots \ldots \ldots \ldots \ldots \ldots \ldots \ldots \ldots \ldots$

Figure 2.33- a) The 3 bytes word structure used by the NMR Bruker system. b) The 4 bytes word of the workstations structure for a positive number conversion and

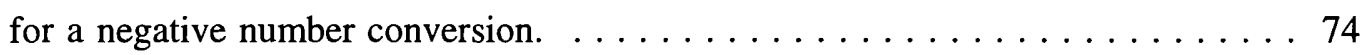

Figure 2.34- The Multi-slice Multi-echo pulse sequence used in the experiments. . . . 76

Figure 3.1 - The NMR spin-echo sequence and the electrical current pulses. . . . . . . 81

Figure 3.2-The net magnetisation in the rotating frame.(a)The phase shift produced by main field inhomogeneities;(b) and (c)The phase shifts produced by a PN and NP current pulse sequence, respectively. . . . . . . . . . . . 85

Figure 3.3 - Phase wrapped image of a transverse section of a cube (slice width

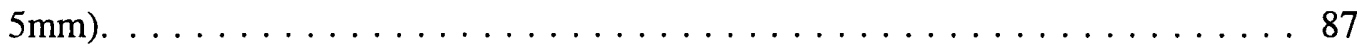

Figure 3.4 - Phase wrapped image profile along a horizontal centre line of figure 3.3.

Figure 3.5 - The difference angle between two complex vectors $\ldots \ldots \ldots \ldots$. . . . 89

Figure 3.6- Artifacts produced during the process of phase unwrapping. . . . . . . . . 90

Figure 3.7 - The region of $2 \times 2$ pixels and the closed path used to detect noise during

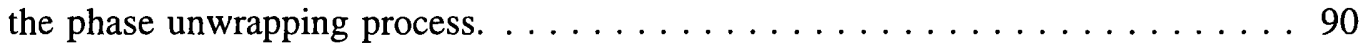

Figure 3.8 - Highlighted noise pixels detected by closed path difference checking. . . . . 91

Figure 3.9 - The flagged points after the phase image in figure 3.8 had been

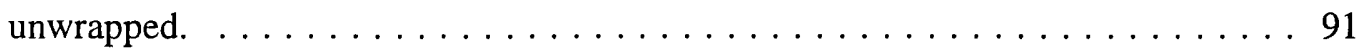

Figure 3.10 - Image magnitude histogram used to define the threshold value during the process of phase unwrapping. $\ldots \ldots \ldots \ldots \ldots \ldots \ldots \ldots \ldots \ldots \ldots \ldots \ldots \ldots \ldots$

Figure 3.11 - Illustration of each step during the phase unwrapping procedure. . . . . 9 95

Figure 3.12 - The closed path used to compute the $I_{y}$ component of current . . . . . . 97

Figure 3.13 - The closed path used to calculate the $I_{x}$ component of current. . . . . . 98

Figure 3.14 - The closed path used to compute the $I_{z}$ current component. . . . . . . . 99

Figure 3.15 - Area normalisation to make the templates to convolute with the NMR phase images. . . . . . . . . . . . . . . . . . . . . . . 100

Figure 3.16 - Image displayed during the image registration. (a)before the image registration (b) after the image registration. . . . . . . . . . . . . . . . . . . . . . 104

Figure 4.1 - Cylindrical phantom used to compare the NMR experimental results with

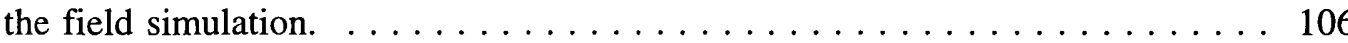


Figure 4.2- Axes reference used to determine the magnetic field arising from the electric current propagating in the inner cylinder. . . . . . . . . . . . . . . . . 107

Figure 4.3 - Top wire position used determine the magnetic field produced by the electric current. . . . . . . . . . . . . . . . . . . . . . . 108

Figure 4.4 - Return wire position to determine the magnetic field produced by the

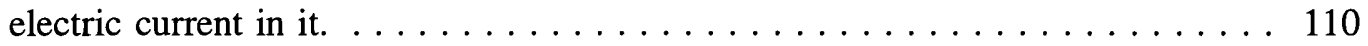

Figure 4.5 - NMR magnitude images to show the slice positions used to measure the current magnetic field. (a) $3 \mathrm{~mm}$ above (b) centre and (c) $3 \mathrm{~mm}$ down. . . . . 111

Figure 4.6 - Current magnetic field components from the simulation. (a) x-direction (b)

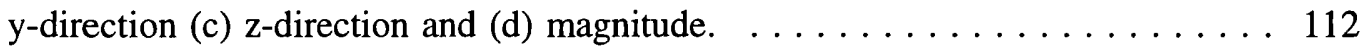

Figure 4.7- Current magnetic field components measured from the NMR experiment. (a) $\mathrm{x}$-direction (b) y-direction (c) z-direction and (d) magnitude. . . . . . . . . 112

Figure 4.8-One pixel wide profile of figures:(a)4.6a dotted line, 4.7a full line;(b) 4.6c

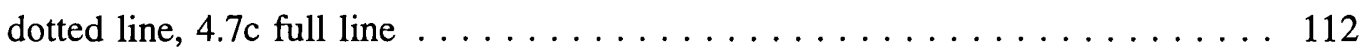

Figure 4.9 - Current image in the y-direction from the simulated magnetic field. . . . . 113

Figure 4.10- Current components from the NMR experiment. (a) $I_{x}$, (b) $I_{y}$ and (c) $I_{z} \ldots 113$

Figure 4.11 - Surface plot of figure $4.10(\mathrm{~b}) . \ldots \ldots \ldots \ldots \ldots$. . . . . . . . . . . . . . . . .

Figure 4.12 - Experimental and simulated horizontal centre profiles of the current images. . . . . . . . . . . . . . . . . . . . . . . . . . . . . . . . . . . . 114

Figure 4.13 - Artifacts in the cylinder current image showed in figure $4.9 \ldots \ldots \ldots 115$

Figure 4.14- Numerical simulated magnetic field components of the cylinder using

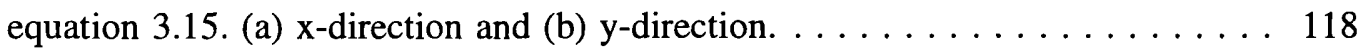

Figure 4.15- Electrical current image calculated from the simulated field component shown in figure 4.14 and the one pixel wide image profile. . . . . . . . . . 119

Figure 4.16- The difference between the simulated field shown in figure 4.6 and in figures 4.14. (a) the $\mathrm{x}$ component and (b) $\mathrm{z}$ component. . . . . . . . . . 119

Figure 4.17- Profiles of the numerical simulated field and that of the NMR experiment . . . . . . . . . . . . . . . . . . . . . . . . . . . 119

Figure 4.18- Simulated magnetic field (a) in the $x$-direction (c) in the $z$-direction. NMR

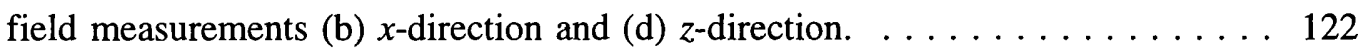

Figure 4.19- Image profiles of a one pixel slice of figure 4.18, (a) from images (a) and (b), (b) from images (c) and (d).

Figure 4.20- Surface plot of the current image computed from the magnetic field measured in the NMR experiment. This experiment was performed keeping the connecting wires parallel to the main field. . . . . . . . . . . . . 12

Figure 4.21- Surface plot of the current image computed only from the simulated 
magnetic field produced by the current in the inner cylinder.

Figure 4.22- Horizontal profiles of the current images from: the field measured in the NMR experiment (full line), simulated cylinder field (dashed line), and simulated cylinder plus wires (dot line) $\ldots \ldots \ldots \ldots \ldots \ldots \ldots$

Figure 4.23- Current images calculated from (a) cylinder plus wires field (b) just the cylinder field (c) from the field measured in the NMR experiment.

Figure 5.1 - Sketch of the cubic phantom constructed to produce a non-uniform current distribution and its axis reference.

Figure 5.2- (a) Slices of the cubic phantom to show the image orientations in relation to $\mathrm{B}_{0}$, and (b) a transverse slice to show the image positions in relation to the

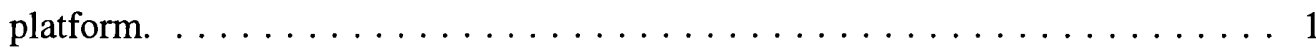

Figure 5.3 - Current magnetic field in the $x-, y$ - and $z$-direction at the three slice positions and a sketch of the platform inside of the frame with the object axis

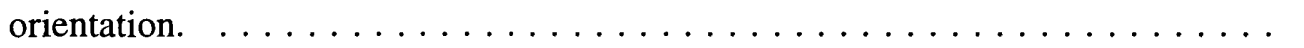

Figure 5.4 - Vector plot of the current induced magnetic field, and a schematic of the platform inside of the cube with the axes orientation. . . . . . . . . .

Figure 5.5 - Component of current in the x-direction. (a) top slice, (b) centre slice and (c) bottom slice.

Figure 5.6 - Current components in the y-direction (a), z-direction (b) and magnitude

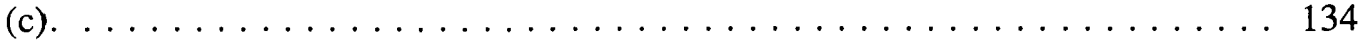

Figure 5.7 - Vector plot of the current in the tomographic plane, and a sketch of the platform to show the holes position and object axis orientation. . . . . . . . 134

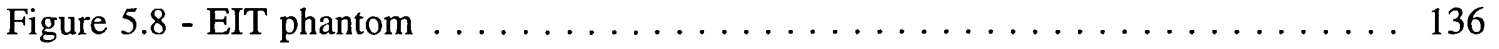

Figure 5.9 - Electrical current distribution inside the EIT tank using adjacent current

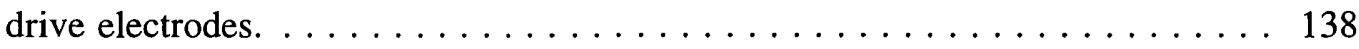

Figure 5.10 - (a) The NMR magnitude images and (b) the corresponding phase images

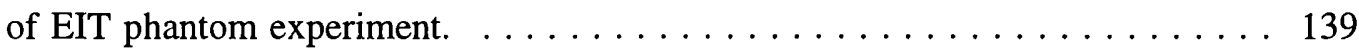

Figure 5.11 - Magnetic field in the EIT phantom experiment at the central slice position . . . . . . . . . . . . . . . . . . . . 140

Figure 5.12 - Current images in the EIT phantom experiment . . . . . . . . . . . 140

Figure 5.13 - Vertical profile, 10 pixels wide, of the Iz component shown in figure

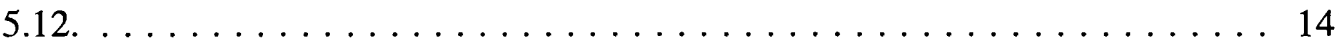

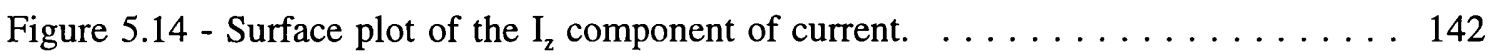

Fig. 6.1 - Effect of pixel size on SNR for ionizing radiation imaging and NMR imaging. For NMR images pixel averaging over a finer array recovers just half of that coarse array. 
Figure 6.2 - The noise background in the NMR phase image (a) makes the object image well defined in the FOV, (b) horizontal image profile. . . . . . . . . . . 146

Figure 6.3 - Phase standard deviation and SNR vs echo-time. (a) inner cylinder (b) out

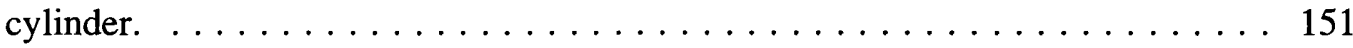

Figure 6.4 - Current standard deviation and $\langle\mathrm{M}>/ \sigma(\mathrm{M})$ vs echo-time. . . . . . . . . 154

Figure 6.5- The error caused by miss-registering the images during the image processing. (a) shift up and down, (b) shift left and right. . . . . . . . 155

Figure 6.6 - The Rician distribution for several SNR and the corresponding mean. . . 157

Figure 7.1- Frame to hold the piglet's head inside the magnet during the NMR

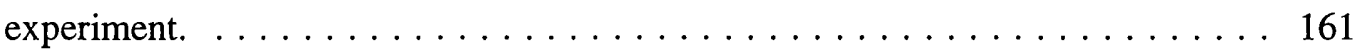

Figure 7.2 - No current NMR magnitude images of the top, centre and bottom slices for the three orientations $\mathrm{x}, \mathrm{y}$ and $\mathrm{z}$ of the head in relation to the main magnetic field. . . . . . . . . . . . . . . . . . . . . . . . . . . . . . . . . . . . . . . . 164

Figure 7.3- Images of the current magnetic field components $B_{x}, B_{y}, B_{z}$ and $B_{m a g}(a)$ for the positive current $(b)$ for the negative current. . . . . . . . . . . . 165

Figure 7.4- Current magnetic field vector plot using figure $7.3\left(B_{x}\right.$ and $\left.B_{z}\right)$. (a) positive current (b) negative current. . . . . . . . . . . . . . . . . . . . . 165

Figure $7.5-I_{x}$ current component, (a) for the positive current and (b) for the negative

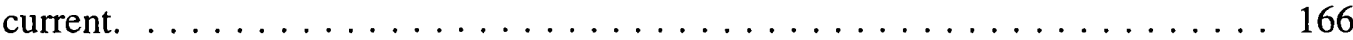

Figure 7.6- $I_{y}$ current component images in the top, centre and bottom slices. First row for the current in the positive y-direction and the other for the current in the

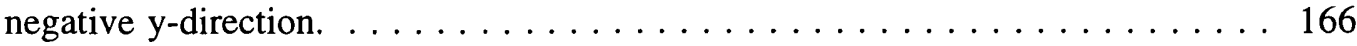

Figure $7.7-I_{z}$ current component images. (a)for the current in the positive y-direction

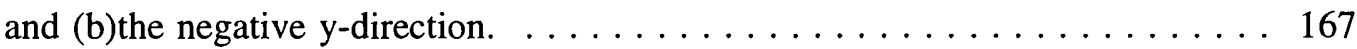

Figure 7.8- Current vector plot. (a) for the PN current and (b) for the NP current. . . . 167 Figure 7.9 Electrical current magnitude image. (a) for the PN current and (b) for the NP current. . . . . . . . . . . . . . . . . . . . . . . . . . . . . . 168

Figure 7.10- ROIs defined to determine the total $\mathrm{I}_{\mathrm{y}}$ current in the images shown in

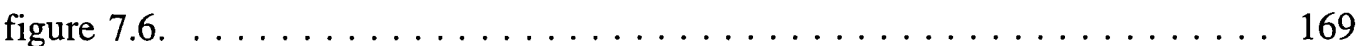

Figure 7.11- (a) and (c) NMR images used to adjust the slice position during the experiment. (b) and (d) current images for respectively the positive and negative current applied during experiment. . . . . . . . . . . . . . . . 171

Figure 7.12 - The mechanism to adjust the head position inside of the magnet . . . . 173

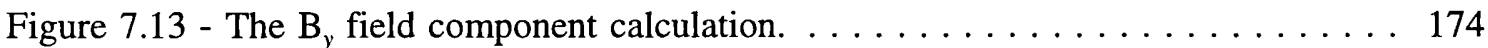

Figure 7.14 -NMR images to show the orientation of the piglet in relation to the NMR magnetic field $\mathbf{B}_{0}$. (a) before (b) after image registration. . . . . . . . . 175 
Figure 7.15- Current magnetic field images (a)positive current experiment (b)negative

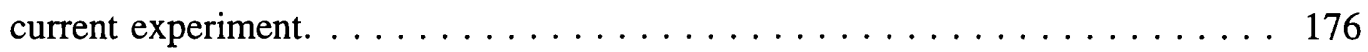

Figure 7.16- (a) and (d) NMR images, (b) and (e) positive current, (c) and (f) negative current, respectively top and centre slices. . . . . . . . . . . 177

Figure 8.1- Sagittal image of the piglet head to approximately show the positions of the

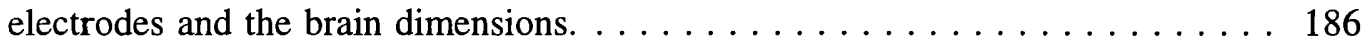

Figure 8.2 - Position of the copper conductors in the frame holding the piglet head (one of the front support bars is shown cut away for clarify) $\ldots \ldots \ldots \ldots \ldots 187$

Figure 8.3 - NMR magnitude images of the "in vivo" experiment. (a) before image

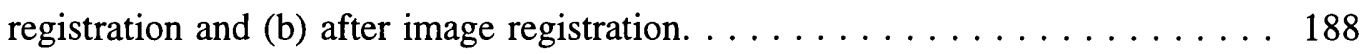

Figure 8.4- The NMR images acquired with the $x$-axis parallel to $\mathbf{B}_{0}$. (a)top (b)centre and (c)bottom slices. . . . . . . . . . . . . . . . . . . . . . . . . . . . 189

Figure 8.5 - NMR phase images acquired with the x-axis parallel to $\boldsymbol{B}_{\boldsymbol{0}}$ to show the effect of the electrical current. (a) wrapped phase and (b) unwrapped phase. . . 190

Figure 8.6 - Electrical current magnetic field at the position of the centre slice. . . . . 191

Figure 8.7 - $I_{x}$ current component in the top (a), centre (b) and bottom (c) slices. . . . 191

Figure 8.8 - (a) $\mathrm{I}_{\mathrm{y}}$, (b) $\mathrm{I}_{\mathrm{z}}$ and magnitude current components. . . . . . . . . 192

Figure 8.9 - Magnitude images of the experiment performed after death . . . . . . . . 194

Figure 8.10 - Phase wrapped and unwrapped images acquired with the $\mathrm{x}$-axis parallel

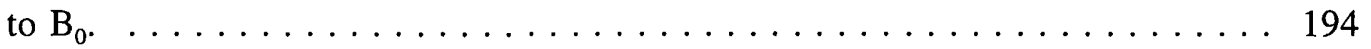

Figure 8.11 - Electrical current magnetic field at the position of the centre slice. . . . 195

Figure $8.12-I_{x}$ current component in the after death experiment . . . . . . . . . 195

Figure 8.13- Current images (a) $I_{y}$ and (b) $I_{z}$ components, and (c) the magnitude calculated using the $I_{x}$ component in the centre slice, figure 8.12 . . . . . . 196

Figure 8.14 - The total current distribution in the head before and after death . . . . . 199

Figure 8.15 - Diagram of the estimated resistivity of the brain and extracerebral layers. Values given are in ohm centimetres (from Holder, 1993b). . . . . . . . 202

Figure 8.16 - Current vector plot (a) for the "in vivo" experiment (b) for the "in vitro" experiment.

Figure 8.17 - Changes in deoxyhaemoglobin $(\mathrm{Hb})$, oxyhaemoglobin $\left(\mathrm{HbO}_{2}\right)$ and the total haemoglobin when the head is turned from the centre position to the left side. . 204

Figure 8.18 - Changes in deoxyhaemoglobin $(\mathrm{Hb})$, oxyhemoglobin $\left(\mathrm{HbO}_{2}\right)$ and the total changes in these compounds concentration when the head is tipped from the centre position to the right side. . . . . . . . . . . . . . . . . . . 205

Figure 8.19 - Median section of the head of a four week old pig; the nasal septum has been removed $($ Sack, 1982) . . . . . . . . . . . . . . . . . . . . . . . . 207 
Figure 8.20 - Top view of a piglet skull $\ldots \ldots \ldots \ldots \ldots \ldots \ldots \ldots$

Figure 9.1 - The new RF pulse sequence to measure low frequency AC current. . . . . 214

Figure 9.2 - NMR magnitude image of the EIT phantom with the plaster of Paris

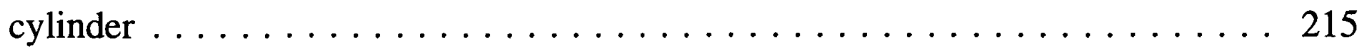

Figure 9.3 - Current components inside the plaster cylinder placed in the centre of the EIT phantom. . . . . . . . . . . . . . . . . 216

Figure 9.4 - Current profiles with and without the plaster of Paris annulus in the EIT

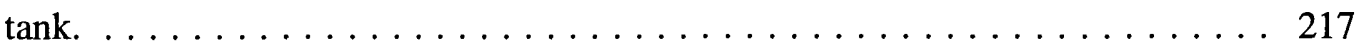

Figure C.1 - Block diagram of the Current Supply and the Current Pulse and Trigger Control . . . . . . . . . . . . . . . . . . . . . . . . . . . . . . . . 220

Figure C.2 - The controllable constant current supply. $R_{s}$ is used to sample the applied current. $P_{1}$ is used to control the current amplitude . . . . . . . . . . . . 221

Figure C.3 - Current direction control. Four optical SCRs are used to control the current direction.

Figure C.4 - Signals to control the current pulses and NMR trigger. (a) NMR trigger, (b) NMR $90^{\circ}, 180^{\circ}$ and readout gradient pulses, (c) (d) (e) and (f) signals to control the current supply. 


\section{List of Tables}

Table 1.1 - Tissue resistivity values (summary from Barber and Brown (1984)) . . . . 28

Table 4.1 - Noise analyses results to quantify the standard deviation in the current image. . . . . . . . . . . . . . . . . 116

Table 4.2 - Noise analyses results to quantify the standard deviation in the current image. . . . . . . . . . . . . . . . . . . . . . . . 126

Table 6.1 - Results for a region of 36 pixels within the inner cylinder. ${ }^{17} \ldots \ldots \ldots 151$

Table 6.2 - Results for a region of 36 pixels within the out cylinder ${ }^{17} \ldots \ldots \ldots \ldots 151$

Table 6.3 - Current noise predicted with equation (6.28) and measured. . . . . . . . 154

Table 7.1 - Surface integration results for the top slice. . . . . . . . . . . 170

Figure 7.2 - Surface integration results for the centre slice. . . . . . . . . . . . 170

Figure 7.3 - Surface integration results for the bottom slice. . . . . . . . . . . . 170

Table 7.4- Surface integral per region for the second experiment. The regions are

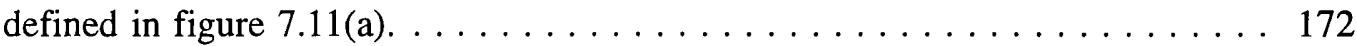

Table 7.5 - Surface integration results for the top slice. The ROIs are defined in figure

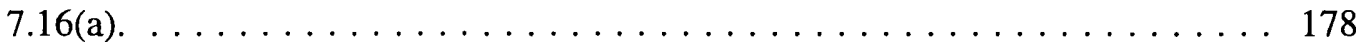

Table 7.6 - Surface integration results for the centre slice. The ROIs are defined in figure $7.16(\mathrm{~d}) . \ldots \ldots \ldots \ldots \ldots \ldots \ldots \ldots \ldots \ldots \ldots \ldots \ldots \ldots \ldots \ldots \ldots$

Table 7.7 - Results of the noise analysis in the in vitro piglet experiments. . . . . . 179

Table 8.1 - Results for the live animal experiment. . . . . . . . . . . . . . . 193

Table 8.2 - Results for the dead animal experiment. . . . . . . . . . . . . . . . 197

Table 8.3 - Results of the noise analysis. . . . . . . . . . . . . . . . . 198

Table 8.4 - Changes in chromophore concentrations in the brain when the head is turned

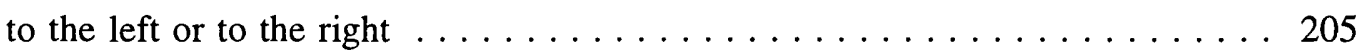




\section{Acknowledgements}

I sincerely thank my supervisor Prof. David Delpy for his constant support, guidance and friendship during the course of this work. His expertise in different fields contributed in many ways to the work presented here. I want also to express my gratitude to Dr. John Thornton for his many suggestions and constant help with the NMR system; Dr. Matthew Clemence for his enthusiastic comments about this thesis; Dr. Clare Elwell for the NIRS measurement and analysis; David Kirkby for suggestion about the design of the current supply; Denzil Booth and Jose Péz for their many lectures on how to use the machinery in the workshop and help with the assembly of the phantoms and Dr. John Wyatt for helping with the clinical aspects of the experiments. On the NMR side I thank Prof. Roger Ordidge for his lectures on advanced NMR topics and Dr. Ernest Cady, Gaby Pell, Marzena Arridge, Mohamed Elsayed, Patricia D'Souza, Richard Aldridge, Shonit Punwani, all of whom have helped me in the NMR aspects of this work. Other members of the department have provided stimulating suggestions including: Christine Sweeting, David Hall, Dave Plummer, Feng Gong, Jeremy Hebden, Joanne Mathias, Kaveh Nahid, Louise Lunt, Mark Cope, Martin Schweiger, Matthias Kohl, Michael Firbank, Mike, Conway, Paul Beard, Paul Ostro, Rebecca Simpson, Roger Springett, Russell Watson, Steven Matcher, Tim Mills. I must also include here Arnulfo Martinez-Davalos and Mercedes R. Villafuerte, both ex-PhD students here in the Medical Physics Department.

I also would like to thank Dr. David Holder's EIT group in the Physiology Department here at UCL for providing the gels, sponges and plaster of Paris materials used in some of the phantom experiments.

In Brasil, I must express my thanks to CEFET-PR (my employer) and to the CAPES Agency for sponsoring my studies. A special thank must go to Dr. Pedro M. Gewehr for his friendship and his faith in my academic skills.

Thanks must also go to my parents Stefano and Ide Gamba and to my uncles Abramo and Dorina Giacomette for all their encouragement in my academic life.

Finally, many, many thanks must go to Rosane who, with no hesitation, left everything to come to London and share with me the difficult and exciting task of completing a Ph.D. whilst living abroad. 


\section{Introduction}

This thesis presents for the first time, images of the electrical current distribution inside the head, resulting from a DC current applied through surface electrodes. The electrical current distributions were measured both in vitro and in vivo using a nuclear magnetic resonance (NMR) technique suggested by Joy et al. (1989). The motivation for these studies is related to an increasing interest in the application of electrical impedance tomography (EIT) to produce images of electrical impedance changes in brain tissue (Holder, 1992; Holder, 1993b; Boone et al., 1994, McArdle et al., 1993, Boone (1995)).

EIT is a relatively new imaging technique which may be applied as a diagnostic tool. The contrast information displayed by EIT is proportional to the electrical impedance of tissue, measured through a sequence of electrodes placed around the surface of the body. An electric current is applied between two electrodes and the potential difference among the other electrodes is measured. The major advantages of EIT over other imaging systems such as CT and NMR, are its time resolution, about $40 \mathrm{~ms}$ ( 25 frames may be collected per second) and the fact that it is potentially both portable and low cost. The main limitation is its spatial resolution of about $15 \%$ of the electrode array diameter (Holder, 1992; Holder and Brown, 1993).

A major problem with the EIT technique is the difficulty in determining the region inside the body which is probed by the electrical current. Indeed, the structures of the human body have a very complex structure and organization, all of which help to determine how the electrical current propagates. The variety of tissue types makes the medium completely non-uniform and anisotropic. Therefore, it is virtually impossible to predict exactly how the electrical current propagates inside the body.

Previous researchers have unsuccessfully tried to produce impedance images of the brain using the Sheffield EIT system developed by Barber and Brown in 1987. The reason for the lack of success is generally attributed to the relatively low conductivity of the skull which stops any current applied on the surface of the head from reaching the brain. It has also been shown that the EIT images of the head obtained with scalp electrodes distort the brain towards to the centre of the image domain making the results difficult to interpret (McArdle et al., 1993; Holder, 1992).

It has been suggested that the use of specific algorithms to reconstruct EIT 
images of the head, which correct for the effect of the current distribution in the skull, might be able to improve the EIT brain images (Holder, 1992). However this is still under investigation and it has yet to produce conclusive results (Bayford, in press).

Recently, a technique to measure electrical current distribution using NMR was suggested (Joy et al., 1989). This technique uses NMR data to compute images of the electrical current applied to the medium. Since NMR is non-invasive, this technique opens up new possibilities for studying the electrical current distributions inside the body.

Thesis aim: the aims of the work described in this thesis were threefold. Firstly, to implement the technique to measure electrical current distribution by NMR and to test it with simulations and experiments on phantoms. Also included in this first part was a study to determine how the NMR noise affects the final current images. Secondly, by performing in vitro experiments in the head of dead animals, to verify if this technique is actually able to measure the electrical current distribution in the heterogeneous tissues of the head, and to implement an experimental methodology that could be applied in an in vivo experiment. Third, to perform an in vivo experiment on an anaesthetised animal to measure the electrical current distribution in the head of a living subject. Hopefully with this technique implemented, and with the results obtained, new a priori information might be obtained which could be incorporated in the image reconstruction algorithms and hence may help to improve the EIT images.

\section{Thesis Organisation}

Chapter 1 contains a concise review of topics related tissue electrical impedance and EIT.

Chapter 2 presents a review of the major points of NMR theory that are relevant to the process of measuring electrical current. The theory of NMR image reconstruction is described and the algorithms implemented to reconstruct and phase correct the NMR images are then explained.

Chapter 3 concentrates on the process of current calculation from the NMR data. A brief review of the three proposed methods found in the literature for the measurement of electrical current by NMR is presented. The theory of the actual technique implemented to calculate the electrical current images from the NMR images is then developed. This chapter finishes with a detailed description of the algorithms 
implemented to register the NMR images, to unwrap the NMR phase images and to calculate the electrical current.

The theoretical simulations and experiments performed to validate the electrical current measurement technique are presented in chapter 4. The experimental methodology developed during these simulations and subsequently used in the in vitro and in vivo NMR experiments is also described in this chapter.

Chapter 5 contains the results of an experiment performed with a cubic phantom to measure a three-dimensional current distribution together with results from further experiments with an EIT tank which provided an estimation of the current sensitivity of the technique.

In Chapter 6, NMR noise theory is reviewed and a theoretical and experimental study to understand noise propagation from the NMR data to the current images is performed.

Chapter 7 presents results of three in vitro experiments performed on the heads of new born piglets to make a preliminary study of the electrical current distribution in the head. The aim of these experiments was to test the stereotactic frame used to hold the piglet head inside the NMR magnet and to establish a methodology to be used in the in vivo experiment.

Chapter 8 starts by describing the in vivo experiment, animal preparation and experiment methodology. The current images and the integrated current per region are then presented. A subsequent post mortem experiment, images and results are also shown.

Finally, Chapter 9 summarises the results of all the studies and outlines some proposals for future work. This chapter finishes with a suggestion for a new NMR pulse sequence that might be able to measure low frequency alternating current distribution. 


\section{Chapter 1}

\section{Electrical Impedance Tomography}

\section{Electrical Impedance Tomography}

The aim of this chapter is to give a brief review of topics related to electrical impedance tomography such as cell and tissue electrical models, methods of tissue impedance measurement, values of tissue impedance, and some aspects of techniques applied to impedance image reconstruction.

\subsection{Tissue Electrical Impedance}

\subsubsection{Electric circuit model of tissue}

Tissue consists of an aggregate of cells surrounded by the interstitial fluid. The cell can be idealized as an electrolyte (the cytoplasm) containing a variety of subcellular structures enveloped completely by a thin membrane. The cell membrane thickness is around $10 \mathrm{~nm}$ thick and is composed mostly of lipoprotein (fat plus protein) (Grollman, 1969). The simplest electrical model of the membrane is a capacitance $C_{m}$ in parallel with a resistance $R_{m}$. The cytoplasm may be modeled as a resistance $R_{i}$ and the extracelluar fluids by a resistance $R_{e}$. Figure 1.1(a) illustrates the electrical model of a single cell and figure 1.1(b) its simplified three element model (Webster, 1990).

The electrical impedance of tissue therefore is a complex function and may be represented by a complex series impedance (a resistance in series with a reactance, figure 1.2(a)) or by a complex parallel admittance (a conductance in parallel with a capacitance, figure $1.2(\mathrm{~b}))$.

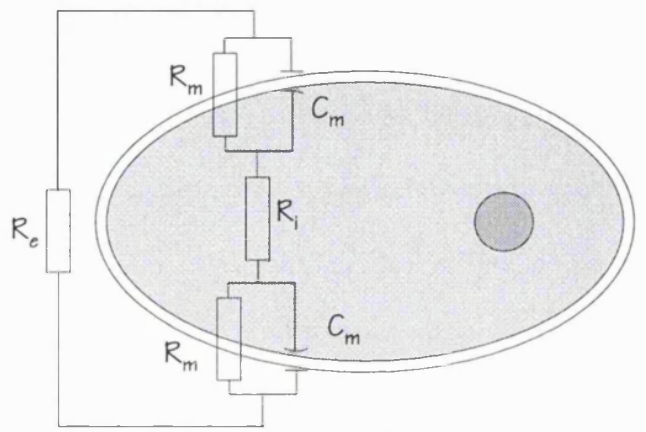

(a)

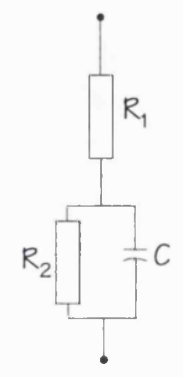

(b)

Figure 1.1 - Electrical model of the cell membrane (from Webster, 1990). 


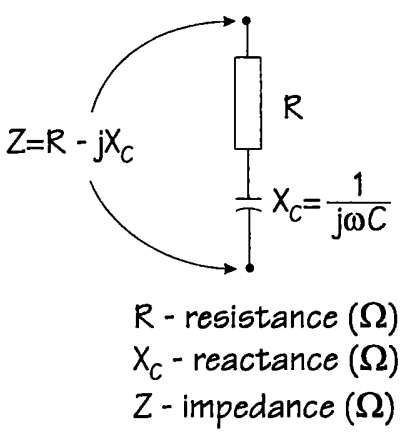

(a)

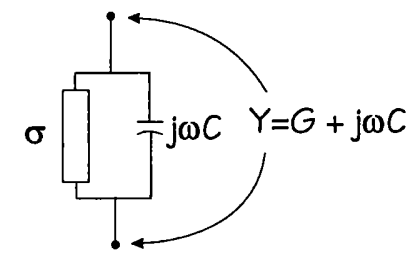

$\sigma-$ conductance $(\mho)$

$C$ - capacitance $(F)$

$Y$-admittance ( $(\mho)$

(b)

Figure 1.2 - (a) series and (b) parallel representations of the tissue complex impedance.

In general the electrical properties of tissue are described in terms of relative permittivity $\varepsilon^{\prime}$ (dimensionless) and conductivity $\sigma$ (mhos per meter $\mho / / \mathrm{m}$ ). The relative permittivity $\varepsilon^{\prime}$ (or dielectric constant) of a material may be interpreted as the ratio between the capacitance of a capacitor with the material as dielectric to the capacitance of the same capacitor but with vacuum as the dielectric ${ }^{1}$. The dielectric property is also referred to as the permittivity $\varepsilon$ and in this case it is defined equal to $\varepsilon=\varepsilon^{\prime} \varepsilon_{0}$ (Hayt, 1989), where $\varepsilon_{0}$ is the permittivity of free space equal to $\frac{1}{36 \pi} 10^{-9} \mathrm{~F} / \mathrm{m}$.

Equations (1.1), (1.2), (1.3) and (1.4) relate the conductivity $\sigma$ and the relative permittivity $\varepsilon^{\prime}$ with the complexes impedance and admittance shown in figure 1.2.

$$
\begin{gathered}
C=\frac{\varepsilon^{\prime} \varepsilon_{0} A}{d}(\mathrm{~F}) \\
G=\frac{\sigma A}{d}(\mathrm{O}) \\
Y=G+j \omega C=\frac{\left(\sigma+j \omega \varepsilon^{\prime} \varepsilon_{0}\right) A}{d} \\
Z=\frac{1}{Y}=\frac{d}{\left(\sigma+j \omega \varepsilon^{\prime} \varepsilon_{0}\right) A}
\end{gathered}
$$

where $A$ is the area of the specimen $\left(\mathrm{m}^{2}\right)$ and $d$ is the depth of the specimen $(\mathrm{m})$.

\subsubsection{The electrical properties of the cell}

Fricke in 1925 first reported the cell membrane capacity as $0.85 \mu \mathrm{F} / \mathrm{m}^{2}$. He made

\footnotetext{
${ }^{1}$ If $C_{1}=\left(\varepsilon^{\prime} \varepsilon_{0} A\right) / d$ is the capacitance of a capacitor with the material as a dielectric and $C_{2}=\left(\varepsilon_{0} A\right) / d$ is the capacitance of a similar capacitor but with vacuum as dielectric, the ratio between $C_{1}$ and $C_{2}$ is the relative permittivity $\varepsilon^{\prime}$.
} 
a wide variety of measurements on different living cells and established this value as a biological constant with extreme values of about 0.5 and $2 \mu \mathrm{F} / \mathrm{cm}^{2}$. The same constancy was not found for the membrane conductance which at rest is equal to $1000 \Omega \mathrm{cm}^{2}$, but depending on physiological changes may vary in a range of 10 to $100 \mathrm{k} \Omega \mathrm{cm}^{2}$ (Cole, 1968). Pethig (1987), assuming a membrane thickness of around $6 \mathrm{~nm}$ and relative permittivity $\varepsilon$ ' equal to 3.0 , estimated the membrane capacitance at $0.44 \mu \mathrm{F}$. Therefore the following typical values may be assigned to the cell membrane in figure 1.1(a), $C_{m}=$ from 0.5 to $1.3 \mu \mathrm{F} / \mathrm{cm}^{2}$ (typical value of $1 \mu \mathrm{F} / \mathrm{cm}^{2}$ ); $R_{m}=$ from $100 \Omega$ to $100 \mathrm{k} \Omega \mathrm{cm}^{2}$; $R_{i}=60 \Omega \mathrm{cm}$.

\subsubsection{Measurement of tissue electrical impedance}

Systems used to measure the electrical impedance of tissue are based on the use of two- or four-electrodes (Burger \& van Dongen, 1961).

- two-electrode technique: The two-electrode method is suitable only for AC measurements. The use of DC in this method is inaccurate because polarization effects at the electrodes produces a counter voltage which changes the measured voltage between the electrodes. The resistance between the electrodes is measured with a Wheatstone bridge circuit, and the conductivity of a cylindrical object can be calculated by (Lytle, 1979),

$$
\sigma=\frac{d}{R A}
$$

where $d$ is the distance between the electrodes (cm), $A$ is the cross-sectional area which is assumed to be constant over the whole length of the object $\left(\mathrm{cm}^{2}\right)$ and $R$ is the measured resistance $(\Omega)$.

- four-electrode technique: The four-electrode method of resistivity measurement can be used for AC as well as DC. It uses a linear array of four equally spaced electrodes, the outer two of which carry an applied current, while the inner two measure the resulting voltage. The four-electrode technique, by providing noncurrent-carrying voltage electrodes, avoids the problem of electrode polarization. The equation from which the unknown resistivity $\rho$ of the tissue is determined is (Rush et al, 1963),

$$
\rho=\frac{2 \pi a V}{I}
$$


where $a$ is the electrode spacing (cm), $I$ is the applied current (A), $V$ is the measured voltage $(\mathrm{V})$ and $\rho$ is the resistivity $(\Omega \mathrm{cm})$. Equation (1.6) applies when the electrodes are on the plane surface of a homogeneous, isotropic conductor of infinite extent.

For media which are anisotropic, with one direction $y$ - of low resistivity $\rho_{l}$ parallel to the bounding plane and with high resistivity $\rho_{h}$ in the $x$ - and $z$-directions, equation (1.6) becomes (Rush et al, 1963),

$$
V=\frac{\sqrt{\rho_{l} \rho_{h}}}{2 \pi a} \frac{1}{\sqrt{\cos ^{2} \theta+\left(\frac{\rho_{l}}{\rho_{h}}\right) \sin ^{2} \theta}}
$$

where $\theta$ is the angle the electrode alignment makes with $x$ - axis.

By aligning the electrodes alternately, for $\theta=0$, equation (1.7) becomes,

and for $\theta=\pi / 2$,

$$
\left.V\right|_{\theta-0}=\frac{\sqrt{\rho_{h} \rho_{l}} I}{2 \pi a}
$$

$$
\left.V\right|_{\theta-\frac{\pi}{2}}=\frac{\rho_{h} I}{2 \pi a}
$$

From equations (1.8) and (1.9) $\rho_{h}$ and $\rho_{l}$ can be found.

Concern has been expressed by many authors regarding the unknown magnitude effects of coupling fluid (typically saline) around the electrodes (Duck, 1990). Even with four electrode systems current may flow through stray capacitances and the electrode contact impedances can cause very large errors, figure 1.3, (Barber and Brown, 1984).

- Current density measurement: When the tissue conductivity properties of a sample are measured with electrodes that are small with respect to the sample size the current distribution is virtually unknown because of differing resistivity of the tissues and fluids and because of the particular arrangement between the current-injection electrodes. Moreover, there is no simple method for accurate measurement of current-density distribution in anisotropic tissue (Geddes and Baker, 1989).

If the conductivity $\sigma$ of a medium is known it is possible to estimate the 

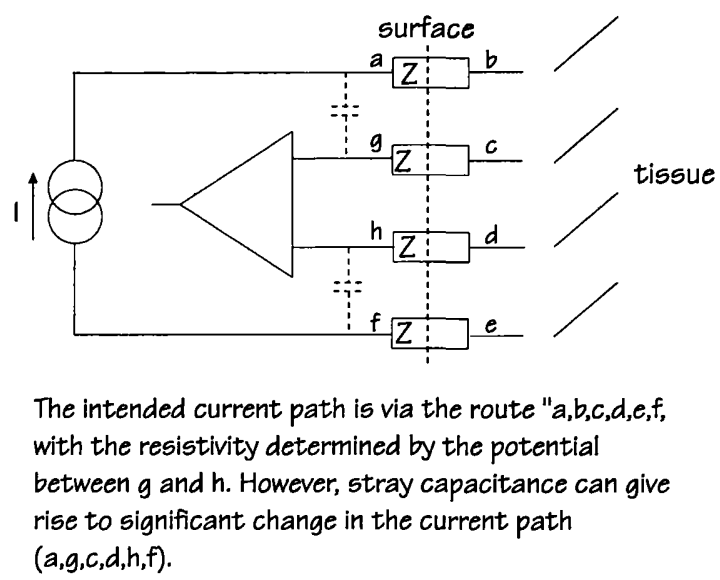

Figure 1.3 - Sketch showing errors which can arise due to electrode impedance $Z$ in a four-electrode system (from Barber \& Brown, 1984).

electrical current distribution $J$ by measuring the potential gradient $\nabla \Phi$ into the medium $^{2}$. The gradient direction may be determined by moving the potential electrodes until the maximum potential is recorded, or by fixing one electrode and measuring the potential difference in the $x$ - $y$ - and $z$-direction. The main problem is to measure the $\nabla \Phi$ without disturbing the medium. The insertion of any insulating or conducting material produces electric field modification. Also, the insertion of electrodes can displace some of the medium, separate structures, and in addition may produce cell damage which releases fluids of differing conductivities. These effects may be minimised by using very small electrodes, but this increases the electrode impedance significantly requiring a voltage amplifier with a very high input impedance to avoid drawing current from the medium. Some probes used to measure the three components of current density were described by Baker and Geddes (1970), all them require the insertion of electrodes into the tissue and measure the current density in a limited region of a biological specimen.

- Guard ring electrode system: For a uniform and isotropic medium the current spreads beyond the electrodes as in figure 1.4(a). To achieve a uniform current distribution under the electrode a concentric guard-ring electrode may be applied. Basically the potential of the guard-ring electrode (figure 1.4(b)) is maintained at the potential of the centre electrode used for the current measurement. The current flowing in the guardelectrode is not measured; it merely aids in providing a uniform current-density distribution around the main current path in the specimen. However, even the guard-

${ }^{2} J=\sigma E$ and $E=-\nabla \Phi$. Therefore $J=\sigma \cdot(-\nabla \Phi)$. 
electrode scheme would not work in a medium like in vivo tissue which is not uniform and is also anisotropic (Geddes and Baker, 1989; Webster, 1990).

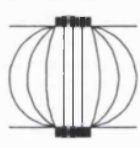

(a)

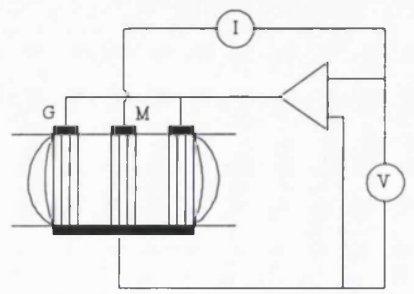

(b)

Figure 1.4 - (a) Current spread in a conductor, and (b) Current spread with a guard electrode maintained at the same potential as the measuring electrode M (from Geddes \& Baker 1989).

\subsubsection{Values of conductivity of tissue at low frequencies}

Figure 1.5 is an illustration of how the current propagation path changes as the frequency increases. At low frequencies, the cell membrane has a high resistance and, the current travels through the extracellular fluids around the cell. At high frequencies, the capacitance of the cellular membrane short circuits the resistance of the membrane and the current lines pass uniformly through the tissue structure.

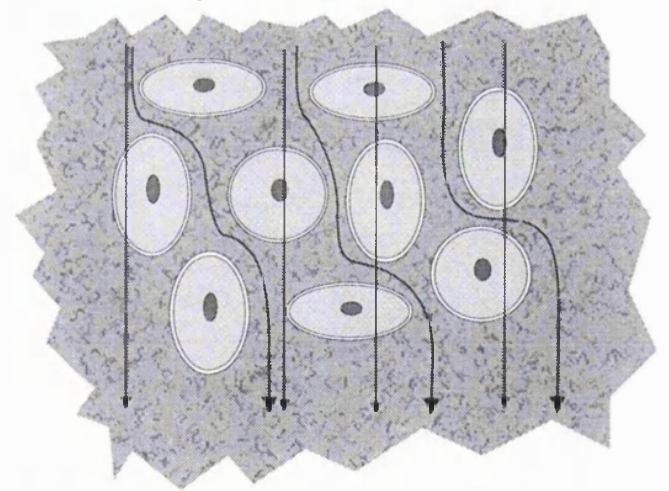

Figure 1.5- At high frequencies the current travels in the straight lines and at low frequencies the current travels longer paths through only the extracellular fluid (from Webster, 1990).

The relative permittivity $\varepsilon$ ' of biological tissue typically decreases with increasing frequency in three major steps, which are designated as the $\alpha, \beta$ and $\gamma$ dispersions, as shown in figure 1.6. The $\alpha$-dispersion is generally considered to be associated with interfacial polarizations at the membrane. The $\beta$-dispersion is associated with two effects namely capacitive shorting-out of the membrane and rotational relaxations of biomacromolecules. The $\gamma$-dispersion arises from the water relaxation in tissue (Pethig, 1987). 


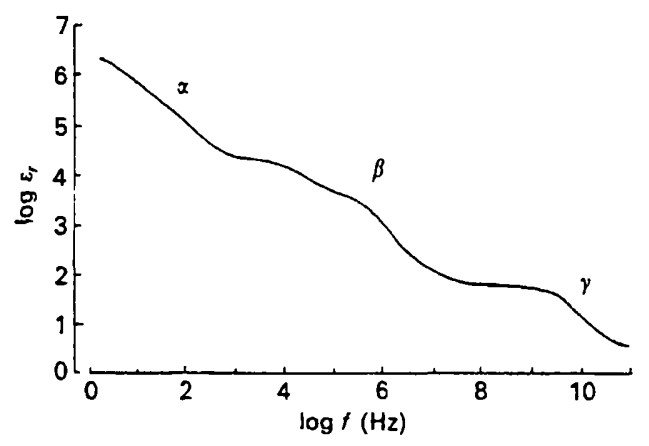

Figure 1.6 - An idealised plot of the frequency variation of the relative permittivity for a typical biological tissue (from Pethig, 1987).

Most of the data about the electric properties of tissue have been obtained by the two- or four-electrode methods applied on animals or excised organs. For in vivo measurements in general the data in the literature refer to body section such as arm, thorax etc. For example, Rush et al. (1963) have studied the resistivity of liver, heart, lung, fat, skeletal muscle and thorax in a set of animals together with some measurement in humans such as skeletal muscle, thorax and blood. They developed a set of probes to match the conditions for equations (1.6), (1.8) and (1.9). Burger and van Dongen (1961) made some measurements in the human arm with plate electrodes. They placed two current-electrodes on the wrists of the subject in order to make the distance between them so large that the conduction over the skin could be neglected and hence they considered the current density in the tissue would be uniform. The voltage electrodes were applied on the forearm at a known distance from one another. By assuming that the arm is a circular cylinder they used equation (1.5) to compute the arm resistivity. They repeated the measurements on nine different persons and an average of $240 \Omega \mathrm{cm}$ $\pm 5 \%$ was measured. Since different amounts of subcutaneous fat existed in the arms of the individuals, they concluded that fat had no influence on the measurement.

Many others workers have made similar measurements (eg. Plonsey and Barr (1982); Geddes and Baker (1967); Witsoe and Kinnen (1967); etc ...), however there are large discrepancies in the literature values of the tissue resistivity. Mainly these arise because of the great difficulties of making in vivo measurements and the problems of preserving tissue for in vitro measurements. A summary of the electrical resistivity values is given in table 1.1 (Barber and Brown, 1984).

Pethig (1987) reported the following results for the electrical properties of several 
tissues over a wide range frequencies: in general comparing the relative tissue permittivity values all tissues tend to follow the same dependence with frequency, with very large values for frequencies below $10 \mathrm{kHz}$. For frequencies between $100 \mathrm{kHz}$ and $100 \mathrm{MHz}$ there are very large changes in dielectric properties associated with the capacitive nature of the cell membranes. For frequencies above $100 \mathrm{MHz}$ the dielectric properties are mainly associated with tissue water content.

Table 1.1 - Tissue resistivity values (summary from Barber and Brown (1984))

\begin{tabular}{|c|c|}
\hline Tissue & Resistivity ( $\Omega \mathrm{m}$ ) \\
\hline Cerebrospinal fluid & 0.65 \\
\hline Plasma & 0.66 \\
\hline Blood & 1.5 \\
\hline Liver & $3.5-5.5$ \\
\hline Human arm & $\begin{array}{l}2.4 \text { (longitudinal) } \\
6.75 \text { (transverse) }\end{array}$ \\
\hline Skeletal muscle & $\begin{array}{c}20.5 \text { (transverse) } \\
1.38 \text { (longitudinal) }\end{array}$ \\
\hline Cardiac muscle & $\begin{array}{l}3.68 \text { (longitudinal) } \\
28.02 \text { (transverse) }\end{array}$ \\
\hline $\begin{array}{l}\text { Neural tissue } \\
\begin{array}{l}\text {-grey matter } \\
\text { - white matter }\end{array}\end{array}$ & $\begin{array}{c}5.8 \\
2.84 \\
6.82\end{array}$ \\
\hline Lung & 7.27(full inspiration)- 23.63 (full expiration) \\
\hline Fat & 27.2 \\
\hline Bone & 166 \\
\hline
\end{tabular}

\subsection{Electrical Impedance Tomography}

In Electrical Impedance Tomography (EIT) electrical current are inject into a subject and from the voltage measured on the surface, or boundary, a cross-sectional image of resistivity, or conductivity, distribution is reconstructed. The first published papers on EIT started in the seventies (Swanson, 1976; Henderson et al., 1976; Price, 1979, etc.) and in 1983, Barber and Brown used the terminology Applied Potential Tomography (APT) to describe their system which was able to produce image of the resistivity distribution of a human arm. Many works may be found about EIT and with the commercialization of the Sheffield instrument many clinical applications and physiological studies have been carried out (Holder and Brown, 1993). 


\subsubsection{The EIT governing equations}

Consider the situation where an alternating current is applied to a dielectric medium. The current is considered to be of low frequency (less than $10 \mathrm{MHz}$ ) so that the equations of static flow can be used to describe the electrical current. The relation between the electrical current density $\boldsymbol{J}\left(\mathrm{A} / \mathrm{m}^{2}\right)$ and the electrical field $\boldsymbol{E}(\mathrm{V} / \mathrm{m})$ is $\boldsymbol{J}=\sigma \boldsymbol{E}$, where $\sigma$ is the conductivity of the medium measured in mhos per meter $(\widetilde{J} / \mathrm{m})$. The continuity equation is $\nabla \cdot J=-\partial \rho_{\nu} / \partial t$, where $\rho_{\nu}$ is the charge density distribution $\left(\mathrm{C} \mathrm{m}^{-3}\right)$ and the divergence operator is equal to $\nabla \cdot=\frac{\partial}{\partial x}+\frac{\partial}{\partial y}+\frac{\partial}{\partial z}$. From these two equations and the relation between electrical field $\boldsymbol{E}$ and the scalar potential $\Phi$, $E=-\nabla \Phi$, the resistivity form of the Poisson equation is obtained,

$$
\nabla \cdot[\sigma(-\nabla \Phi)]=-\frac{\partial \rho_{v}}{\partial t}
$$

The electric flux density of a dielectric medium is given by $\boldsymbol{D}=\varepsilon_{0} \boldsymbol{E}+\boldsymbol{P}$ (Hayt, 1989), where $\varepsilon_{0}$ is the free space permittivity and $\boldsymbol{P}$ is the material polarization or dipole momentum per unit volume. The first term on the right is the contribution that would exist if the electric field were in free space, and the second is the effect of the polarization of the material. The polarization produced by the electrical field in the material depends upon the material properties, which may be divided into four classes: homogeneous if the electric properties do not depend upon position, isotropic if the response of the material is the same for all directions of the electrical field vector, linear if the ratio of the response of $\boldsymbol{P}$ to the field $\boldsymbol{E}$ is independent of the amplitude, and timeinvariant if the material properties do not change with time.

When considering body tissues obviously they are not homogeneous since this is the main reason for EIT imaging, and unfortunately they are not isotropic since the muscle cells have a lower resistivity along the fibres than across the fibres. Because low current are used in EIT tissue linearity can be assume (Barber and Brown, 1984). Thus, for an isotropic, linear material the polarization is proportional to the field intensity and is equal to $\boldsymbol{P}=\varepsilon_{0} \chi \boldsymbol{E}$, where $\chi$ is a dimensionless quantity called the electric susceptibility of the material. The flux density is equal to $D=\varepsilon E$, where $\varepsilon=\varepsilon_{r} \varepsilon_{0}$ and $\varepsilon_{r}$ is the relative permittivity, equal to $l+\chi$. If the medium is anisotropic, the dielectric 
properties cannot be described in terms of a single permittivity parameter. Each component of $\boldsymbol{D}$ may be a function of every component of $\boldsymbol{E}$ and $\boldsymbol{D}=\boldsymbol{\varepsilon} \boldsymbol{E}$ becomes a matrix (Hayt, 1989).

From the Maxwell equation $\nabla \cdot \boldsymbol{D}=\rho_{v}$, the field equation $\boldsymbol{E}=-\nabla \Phi$, and considering the medium as being isotropic and linear,

$$
\nabla \cdot[\varepsilon(-\nabla \Phi)]=\rho_{v}
$$

Expanding the divergence in equations (1.10) and (1.11),

$$
\begin{aligned}
& \sigma \nabla^{2} \Phi+\nabla \sigma \cdot \nabla \Phi=\frac{\partial \rho_{v}}{\partial t} \\
& \varepsilon \nabla^{2} \Phi+\nabla \varepsilon \cdot \nabla \Phi=-\rho_{v}
\end{aligned}
$$

Thus the problem in EIT is to solve (1.12) and (1.13) for $\sigma$ and $\varepsilon$ everywhere inside of the patient, subject to the boundary condition that $\Phi$ is known wherever an electrode touches the skin.

The EIT technique uses low frequencies, Brown and Seagar (1987) have used $51 \mathrm{kHz}$ and Kotre (1989) $20 \mathrm{kHz}$. In biological tissue impedance measurement at frequencies below than $100 \mathrm{kHz}$, the great majority of current travels in the extracellular fluid, which is a resistive medium (figure 1.5). The resistivity component of the complex impedance therefore dominates in impedance measurements (Holder and Brown, 1993). Hence the EIT problem only involves solving equation (1.12).

\subsubsection{Solutions for equation (1.12)}

Equation (1.12) defines the forward problem, which seeks to calculate the voltage distribution resulting from a given resistivity distribution. It is a complicated differential equation and it is impossible to solve it analytically. Therefore, numerical techniques such as the finite element method or finite difference method have to be used. Since EIT seeks to determine a resistivity distribution from a set of voltages measured in the boundary the inverse problem to solve equation (1.12) is more likely to be used. This is a nonlinear problem, as the voltage $\Phi$ is a function of the conductivity $\sigma$.

According to some mathematical studies, because the human tissue is not uniform and mainly because it is anisotropic, the inverse solution of equation (1.12) is not unique (Barber, 1993). This means that numerical techniques may not be adequate 
to reconstruct absolute impedance images from a set of voltages measured at the boundary.

An alternative method for the inverse problem is the backprojection algorithm proposed by Barber and Brown (1983). Because this is the technique applied to most of the EIT clinical data available and because the backprojection is the algorithm used in the commercially EIT system this approach is discussed in the next section.

\subsubsection{Applied Potential Tomography}

Applied potential tomography (APT) was developed as an alternative approach for the nonlinear iterative process of solving equation (1.12). In APT, to map the resistivity distribution a backprojection algorithm adapted from x-ray computed tomography image reconstruction is used. The technique applies 16 electrodes which are equally spaced around the region to be imaged and a alternating current of $51 \mathrm{kHz}$, typically $1 \mathrm{mApp}$ is driven through the body via a pair of adjacent electrodes. This pair of electrodes are called the current electrodes. The potential difference is then recorded between each of the remaining adjacent pairs of electrodes which are called the voltage electrodes. These voltages are then related to the distribution of current within the region near to the electrodes and hence to the distribution of resistance. This process is repeated for the 16 possible independent pair of current electrodes ${ }^{3}$.

The backprojection algorithm is based on the calculated equipotentials considering the medium to be uniform and isotropic. The calculation assumes that each pair of current electrode is equivalent to a dipole localised in the midpoint between the current electrodes. From this assumptions the equipotentials passing through each voltage electrodes are calculated. The voltage difference between each pair of adjacent electrodes is the reference voltage, $V_{\text {ref, }}$, which is indicated in figure 1.7(a) for the selected pair of current electrode.

Consider now that the resistivity at a certain region $R$ between these two equipotentials is altered, figure $1.7(\mathrm{~b})$. Also, consider that this change is small enough

\footnotetext{
3 For $N$ electrodes there are $N-I$ independent drive pairs since the $N$ th pair can be reconstructed from the sum of all the other pairs. The voltage differences between adjacent electrodes are measured. The voltage differences which include the current drive electrodes are discarded, because of the voltage drop across the contact resistance. Therefore $N-3$ voltage differences measurements can be obtained for $N$ electrodes. There is also reciprocity between pairs of current application and of voltage measurements which reduce the number of independent measurement to $N(N-3) / 2$ (Barber and Brown, 1983).
} 

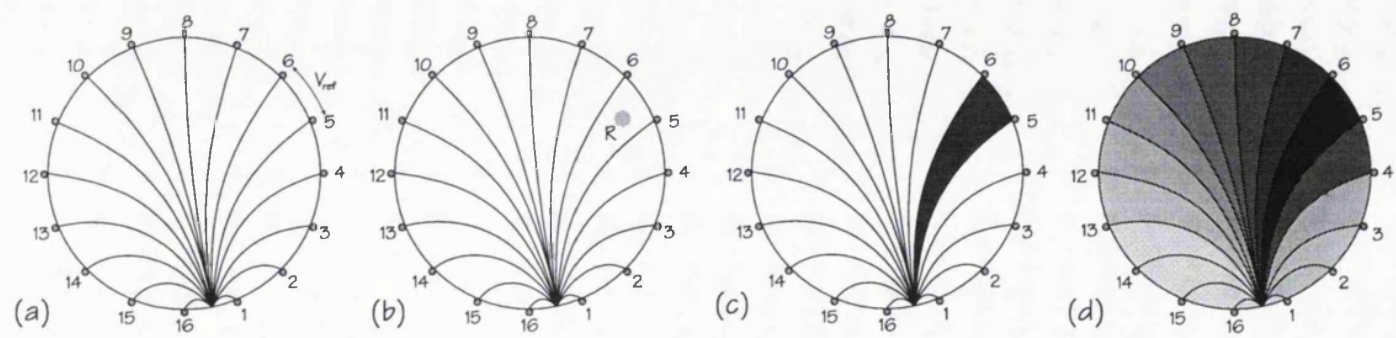

Figure 1.7 - The backprojection image reconstruction

to assume that the isopotentials are kept unperturbed. Now the voltage measured between points 5 and 6 is different from $V_{\text {ref }}$ and since the current source is constant the difference is proportional to the change in the conductivity. The normalised voltage difference is then projected to the entire region between the two isopotentials, figure 1.7(c). Even though this local change in the resistivity affects mainly the region between the equipotentials as shown in figure 1.7(c), all other voltages measured are affected and after a single backprojection the image will look like figure 1.7(d). Therefore for a single drive configuration the backprojection is unable to isolate individual points, but this process is repeated for all 16 current dipole and each backprojection is superimposed to produce the final image. The backprojection process as described above is inaccurate because if the resistivity change occurred near to the electrode the voltage change would be different than if it occurred in the centre. Therefore the backprojection algorithm applies for each pixel a weighting function which depends upon the position of the resistivity change (Barber and Seager, 1987).

In general APT images have a very low spatial resolution mainly because of the limited number of electrodes that can be applied around the region to be imaged and also it is dependent on the position at which the change in the resistivity occurs. Another aspect is the assumption that the current distribution lies within the 2-dimensional image plane, whilst clearly the current spreads out of the plane of interest, making the technique sensitive to off-plane changes in the conductivity. In general, accurate anatomical information can not be obtained from APT images. However much functional information may be obtained.

\subsubsection{The data acquisition system and image resolution}

Many possible electrode arrangements and combinations of applied voltages or 
currents are possible and similarly various measurement configurations can be used to detect the surface signal on the object periphery (neighbouring method, cross method, opposite method, multidifference method, adaptive method, linear array) (Webster, 1990, Barber, 1993). Since the only commercially available EIT equipment is the Sheffield Mark I (Holder and Brown, 1993), which uses the neighbouring method just this measurement technique is discussed here.

The neighbouring method uses an array of electrodes which are attached equally spaced on to the body surface. It is based on the four electrode principle in which the effect of the electrode impedance is greatly reduced by using two electrodes to drive the current inside the region to be imaged and another two to measure the resulting surface voltages.

It has been shown that a set of current distributions which seek to mimic parallel beam x-ray computed tomography, as illustrate in figure 1.8(a), are not sufficiently independent to permit a unique image reconstruction. A better solution for a twodimensional array of equally spaced electrodes is obtained by passing current in turn between all adjacent pairs of electrodes, such as in the figure 1.8(b) (Barber and Seagar, 1987). In this configuration the current flows throughout the circular cross section, with its intensity maximum at the periphery near the current-injecting electrodes and diminishing rapidly towards the opposite side.

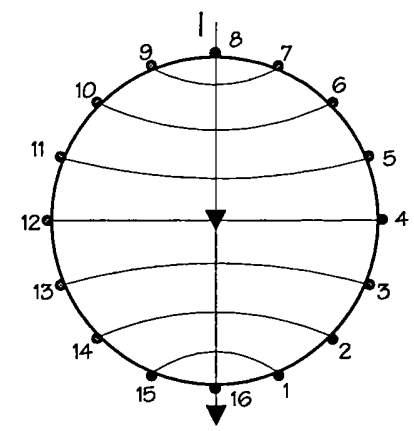

(a)

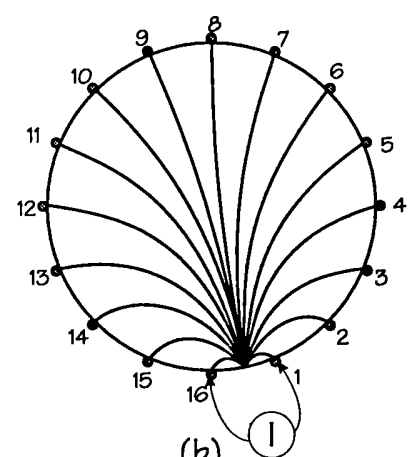

(b)

Figure 1.8 - The isopotentials lines for different current patterns in a uniform conductivity medium.

For $N$ electrodes there are $N-1$ independent drive pairs since the $N t h$ pair can be reconstructed from the sum of all the other pairs. The voltage differences between adjacent electrodes are measured. The voltage differences which include the current drive electrodes are discarded, because of the voltage drop across the contact resistance. Therefore $N-3$ voltage difference measurements can be obtained for $N$ electrodes. There 
is also reciprocity between pairs of current application and of voltage measurement which reduce the number of independent measurement to $N(N-3) / 2$ (Barber and Brown, 1983).

The Sheffield data collection system uses 16 electrodes equally spaced around the body as illustrate in figure 1.8(b). An electrical current of $5 \mathrm{~mA}$ at a frequency of $51 \mathrm{kHz}$ is applied at each pair of neighbouring electrodes and the voltage is measured from all other successive pairs of adjacent electrodes (Brown and Seagar, 1987). Thus a total of 104 independent measurements are obtained. Holder et al. (1993) reports that 104 independent measurements gives a maximum of 104 pixels in an image and a maximum spatial resolution of about $10 \%$ of the electrode array diameter near the edges which decreases towards the centre.

\subsubsection{The main limitations of the EIT}

The major drawback of the current EIT systems compared to other imaging techniques such as X-ray CT and NMR is its poor spatial resolution, which is limited to about $15 \%$ of the electrode array diameter. In saline tanks it has been shown that EIT systems are able to distinguish the image of two object when they were separated by about $20 \%$ of the electrode array diameter (Holder, 1992). It has also been shown that it is able to localize the centre of a single disturbance with an accuracy of $4 \%$ of the electrode array diameter (Holder, 1992).

To improve the image resolution the number of electrodes must be increased. For example, if the number of electrodes is doubled, the number of independent measurements is quadrupled and the spatial resolution should be improved by a factor of two. However, increasing the number of electrodes reduces the signal-to-noise ratio, and the resolution is also limited by three dimensional spread of current so that the improvement in resolution will actually be less than a factor of two (Brown in Holder, 1993). Theoretically, to improve the resolution from $10 \%$ to $1 \%$ of the electrode array diameter the number of electrodes must go from 16 to 128 (Barber and Brown, 1984).

The neighbouring electrode method of data collection has a very non uniform current distribution. Most of the current travels near the peripheral electrodes and hence good sensitivity is obtained just at the periphery (Webster, 1990). Thomas et al. (1993) demonstrated with a cylindrical tank that there is a compression of the EIT images toward the centre, i.e., in moving an object from the centre to the edge, the peak 
impedance increases, whilst the area under $50 \%$ of the peak reduces.

The electrodes are usually attached in a two-dimensional ring, and images are generated on the assumption that the object is two dimensional. But since the object is actually three-dimensional, the current will flow out of the plane in both directions, so that off-plane impedances changes will affect the image. Also, the process used to generate the simple images from the computed isopotentials in a uniform medium may produce some image distortions since in practice the tissue is not homogeneous.

\subsection{Discussion and conclusion}

The last section described the principles and some of the main limitations of the current EIT systems. Even so EIT has many advantages, for instance it is a relatively low cost technique and has a good time resolution (less than $50 \mathrm{~ms}$ per image) which means that it is able to follow physiological changes. Many applications of EIT are under investigation such as the study of the respiratory system and the gastrointestinal tract (Holder and Brown in Holder, 1993). One application of particular interest for this thesis is the possible use of EIT to produce images of the brain. It has been proposed that EIT might be able to provide information about epileptic foci before surgery procedure (Boone et al., 1994) and for early diagnosis of intraventricular haemorrhage in neonates (Murphy et al., 1987).

Previous research so far has however shown that images of changes in the brain impedance are very difficult to obtain with EIT system and scalp electrodes (Holder, 1993b). Although this evidence has largely been based on tests made with the Sheffield EIT system, nobody knows for sure the reason for the poor quality of the EIT images. Impedance models of the head suggested that the main reason is the low conductivity of the skull which stops the majority of the current from reaching the brain. However, due to the difficulties of measuring tissue impedance in vivo these models may not be accurate. So far, most of the data about tissue impedance is based on measurement on excised organs, where the lack of blood and difference in temperature may significantly alter the impedance from its true in vivo value.

Further more, it may also be the case that because of the different layers of tissues, the current may reach the brain region through different paths instead of going straight through the skull, for instance, it may preferentially enter the brain via the ear canals, the optic nerves and the spinal column. The current path will certainly depend 
on the configuration of the current drive electrodes, and in the case of adjacent electrode drive it may be very unlikely for such circuitous routes to be taken but it is not the case for diametrically opposed electrode drive.

Since the medium is three-dimensional, anisotropic and non-uniform, the assumption for the image reconstruction of a starting point of a two-dimensional uniform medium may also be a reason for the poor quality of the EIT images.

Although, it has been shown that no useful images of brain impedance changes may be made with the Sheffield EIT system, scalp electrode research is still being carried out to try to obtain images of absolute impedance (Holder, 1993a).

Therefore with the aim of answering some of these questions, and providing new information that might be useful for the EIT image reconstruction applied to the head, we undertook the task of studying how the electrical current applied onto the surface of the head is distributed within its internal tissues. To do this, a technique to measure electrical current distribution using magnetic resonance imaging (MRI) data was implemented. With this technique it is possible to quantify the current distribution in the head within the main groups of tissues (scalp, skull and brain), and also to determine the probable path taken by the current in going between the surface electrodes. We hope with these measurements to provide useful information that might help the process of EIT image reconstruction of the brain. 


\section{Chapter 2}

\section{Nuclear Magnetic Resonance - NMR}

\section{Nuclear Magnetic Resonance}

The aim of this chapter is to give a brief introduction to NMR theory concentrating on particular aspects relevant to the work to be described later. Also presented here are details of the algorithm implemented to reconstruct and phase correct the NMR images acquired in the experiments to measure the electrical current distribution. The chapter starts with the basic concepts of nuclear resonance, radiofrequency absorption and the rotating frame followed by the spin relaxations and the imaging spin-echo sequence. Finally, the theory of image reconstruction and phase correction is reviewed and the algorithm implement to reconstruct the NMR phase images is described.

\subsection{Principles of Nuclear Magnetic Resonance}

\subsubsection{Angular Momentum and Magnetic Moment}

The angular momentum of an atomic system is inseparable from the magnetic properties of atoms. Electrons and protons are electrically charged and from Maxwell's equation any rotating or circulating charges will give rise to magnetic fields.

Classically, suppose that a proton can be pictured as a rotating sphere of mass $m$ with radius $a$ and uniformly distributed charge $q$, (figure 2.1) (Morris, 1986). Each element of mass traces out a circular path and contributes to the spin angular momentum of the whole sphere. For each element the angular momentum relative to the origin of the coordinate system, is a vector quantity $\boldsymbol{L}$ defined by the equation,

$$
\boldsymbol{L}=\boldsymbol{r} \times \boldsymbol{p}
$$

where $\boldsymbol{r}$ is the position vector of the element of mass relative to the origin, and $\boldsymbol{p}$ is the linear momentum for the particle $(\boldsymbol{p}=m \boldsymbol{v})$. The element of angular momentum of the shaded ring in figure 2.1 is equal to,

$$
\delta L=2 \pi \rho_{m} r^{3} \sin ^{2} \theta \delta \theta \delta r v
$$

where $v$ is the tangential velocity of the ring equal to $(2 \pi r \sin \theta) / t_{r}$ where $t_{r}$ is the revolution period and $\rho_{m}$ is the mass density equal to $(3 m) /\left(4 \pi a^{3}\right)$. 


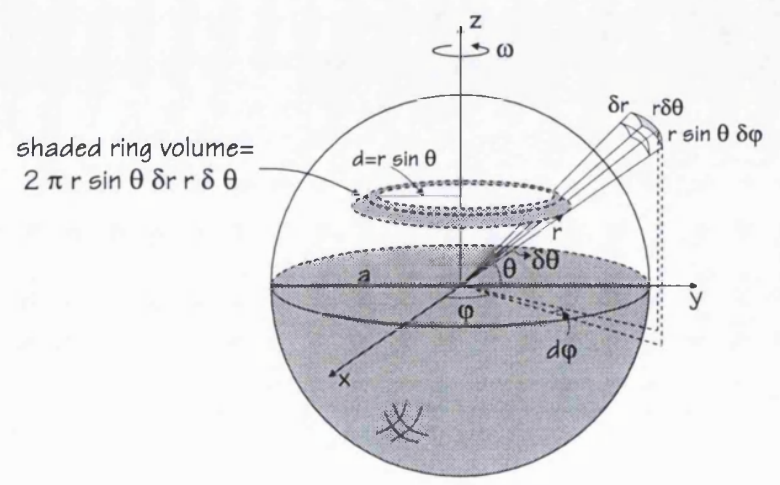

Figure 2.1-Classical model for the estimation of the angular momentum of a proton

Equation (2.3) gives the resulting angular momentum for the whole sphere,

$$
\boldsymbol{L}=\frac{4 \pi^{2} \rho_{m}}{t_{r}} \int_{0}^{2 \pi} \int_{0}^{2 a} r^{4} \sin ^{3} \theta \delta \theta \delta r
$$

Now, since the charge of each element passes a given point on the circle once in every $T=2 \pi r / v$, it is equivalent to a current $i$ given by,

$$
i=\frac{\rho_{e} d v}{t_{r}}=\frac{\rho_{e} d v v}{2 \pi r}
$$

where $\rho_{\varphi}=(3 q) /\left(4 \pi a^{3}\right)$ is the charge density. Such a current loop produces a magnetic field oriented perpendicular to its plane. In elementary electromagnetic theory, it is shown that such a current loop produces a magnetic field which is the same at large distances from the loop as that of a magnetic dipole located at the centre of the loop and oriented perpendicular to its plane. Figure 2.2 illustrates the magnetic dipole for the orbital electron case. For the current loop of area $A$ the magnitude of the orbital magnetic dipole moment $\mu_{l}$ of the equivalent dipole is equal to $\mu_{l}=i \mathrm{~A}$.

In figure 2.1 the ring area is equal to $\pi r^{2} \sin ^{2} \theta$, and the resulting magnetic moment for the whole sphere is equal to,

$$
\mu_{l}=\frac{2 \pi^{2} \rho_{e}}{t_{r}} \int_{0}^{2 \pi} \int_{0}^{2 a} r^{4} \sin ^{3} \theta \delta \theta \delta r
$$

From equation (2.3) and (2.5),

$$
\mu_{l}=\frac{q}{2 m_{p}} \boldsymbol{L}=\boldsymbol{\gamma} \boldsymbol{L}
$$

The constant of proportionality between $\boldsymbol{L}$ and $\mu$ is called the gyromagnetic ratio 


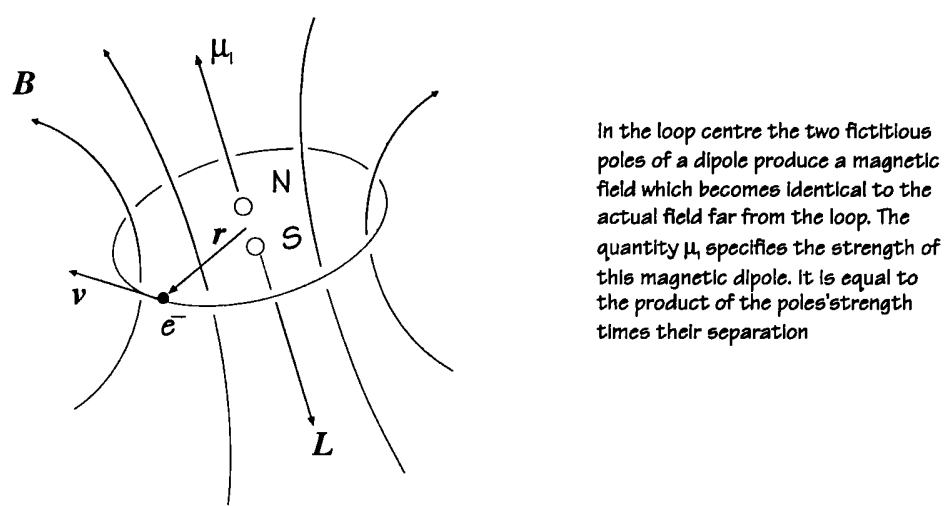

Figure 2.2- The orbital angular momentum $\boldsymbol{L}$ and the orbital magnetic moment $\mu_{l}$ of an electron (from Eisberg, 1985).

$\gamma$, which depends only on the charge to mass ratio of the particle.

To determine correctly the angular momentum of atomic system quantum theory is needed. However since this topic is not relevant to this work, only a brief description of the main equations that determine the NMR net magnetisation is presented here. The separation of the time-independent Schröedinger equation for the one-electron atom system gives rise to three ordinary differential equations. From the solutions of these three equations, three quantum numbers are produced. They are represented by $n, l$ and $m_{l}$ and they are defined as follow: because of its role in specifying the total energy, $n$ is called principal quantum number. Because the azimuthal, or orbital, angular momentum of the atom depends on $l$, it is called azimuthal quantum number, and since when the atom is in an external magnetic field there is a dependence of its energy on $m_{l}$, it is called magnetic quantum number (Eisberg, 1985). Gathered together the quantum numbers are expressed as,

$$
\begin{aligned}
& n=1,2,3, \ldots \\
& l=0,1,2,3, \ldots, n-1 \\
& m_{l}=-l,-l+1, \ldots, 0, \ldots,+l-1, l
\end{aligned}
$$

It can be shown that the quantum numbers $l$ and $m_{l}$ are related to the magnitude $\boldsymbol{L}$ and to its $z$-component, $L_{z}$, by the relation,

$$
\begin{aligned}
& \boldsymbol{L}=\sqrt{l(l+1)} \hbar \\
& L_{z}=m_{l} \hbar
\end{aligned}
$$

where $\hbar$ is the shorthand symbol for $h / 2 \pi$ and $h$ is Planck's constant ( $h=6.63 \times 10^{-34} \mathrm{Js}$ ).

From these equations the angular momentum vector, can assume a range of states 
with the common value $\boldsymbol{L}$. For each of these states the length of the angular momentum vector, in units of $\hbar$ is $L / \hbar=\sqrt{l(l+1)}$. In the same units the $z$-component can assume any integral value from $L_{t} / \hbar=-l$ to $L_{t} / \hbar=+l$ depending on the value of $m_{l}$. Figure 2.3 illustrates the five position states for the case of $l=2$.

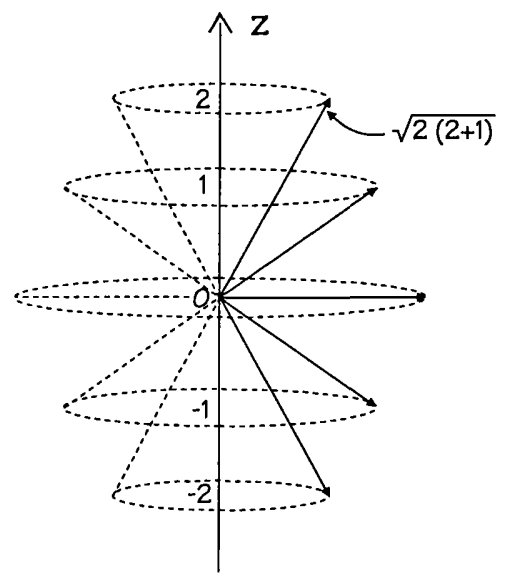

Figure 2.3-Representing the angular momentum vector for the possible states $l=2$. In each state the vector is equally likely to be found anywhere on a cone symmetrical about the z-axis.

In any of these states the angular momentum vector is equally likely to be found anywhere on a cone symmetrical about $z$-axis, and therefore has a definite $z$-component as well as a definite magnitude. The vector does not have a definite $x$ - and $y$-component, but the value of either of these quantities is as likely to be positive as it is to be negative. ${ }^{4}$

If the nuclear spin $l$ is equal to zero, the nucleus would have no angular momentum and hence no magnetic moment: it would therefore not be directly observable by NMR. Such is the case for the common isotopes of carbon ${ }_{6}^{12} \mathrm{C}$ and oxygen ${ }_{8}^{16} \mathrm{O}$. However, many other atoms of biological interest do possess non zero nuclear spin, including ${ }^{1} \mathrm{H}$ (of major interest due to its abundance in biological systems in the form of water), ${ }^{31} \mathrm{P},{ }^{19} \mathrm{~F},{ }^{13} \mathrm{C}$ all of which possess spin $1 / 2$ and ${ }^{23} \mathrm{Na}$ which possesses a spin of $3 / 2$.

However the magnitude $L$ and the $z$-component $L_{z}$ are quantized, no information about the azimuthal orientation $\varphi$ of $\boldsymbol{L}$ can be stated. The $\boldsymbol{L}$ and $L_{2}$ components define a cone shaped region called space quantization. This coincides with the Heisenberg uncertainty principle which states that it is not possible simultaneously determine the exact value of the momentum $p_{x}$ of a particle and also the exact value of its corresponding position $x$. Instead, our precision of measurement is inherently limited by the measurement process itself such that $\Delta p_{x} \Delta x \geq \hbar / 2$ 


\subsubsection{Nuclear precession in a static magnetic field}

Consider the situation where the charged spinning sphere is placed in an applied magnetic field $\boldsymbol{B}_{0}$. The equivalent magnetic dipole moment $\mu_{t}$ of the spinning sphere is represented in figure 2.4 by the two fictitious magnetic poles $\pm q_{m}$ separated by a distance $d,\left(\mu=q_{m} d\right)$. The applied magnetic field creates forces $( \pm \boldsymbol{F})$ acting on the north and south poles of the magnetic dipole equal to,

$$
\boldsymbol{F}=q_{m} \boldsymbol{B}_{0}
$$

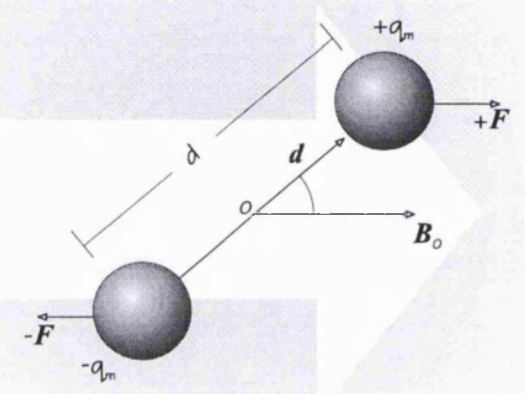

Figure 2.4-Magnetic dipole representing the magnetic moment of the positive spinning charge in Figure 2.I. The magnetic field produces a torque about o.

The forces acting in opposition directions on each magnetic pole produce a net torque $\tau$ about $o$, equal to,

$$
\tau=q \boldsymbol{B}_{0} \frac{d}{2} \sin \theta+q \boldsymbol{B}_{0} \frac{d}{2} \sin \theta=q d \boldsymbol{B}_{0} \sin \theta
$$

where $q d$ is the magnetic dipole moment, acting in the same direction as $\boldsymbol{d}$ (figure 2.4), therefore,

$$
\tau=\mu B_{0} \sin \theta=\mu \times B_{0}
$$

The torque created tends to align the dipole with the field and associated with this torque, there is a potential energy of orientation equal to $E=-\mu_{1} \cdot \boldsymbol{B}_{0}$ (Eisberg, 1985). If there is no way for a system, consisting of a magnetic dipole moment $\mu_{l}$ in a magnetic field $\boldsymbol{B}_{0}$, to dissipate energy, then the orientational potential energy $E$ of the system must remain constant. In these circumstances $\mu_{l}$ cannot align itself with $\boldsymbol{B}_{0}$. Instead $\mu_{l}$ will precess around $\boldsymbol{B}_{0}$ in such way that the magnitude of both vectors and the angle between them remains constant. The precessional motion is a consequence of the fact that according to equations (2.6) and (2.10) the torque acting on the dipole is always perpendicular to its angular momentum, 


$$
\tau=\mu \times B_{0}=\gamma L \times B_{0}
$$

As shown in figure 2.5 , this torque gives rise to a change $d \boldsymbol{L}$ in the angular momentum during a time $d t$. The change $d \boldsymbol{L}$ causes $\boldsymbol{L}$ to precess through an angle $\omega_{0} d t$, where $\omega_{0}$ is the precessional angular velocity. From the diagram,

$$
d \boldsymbol{L}=(\boldsymbol{L} \sin \theta) \omega_{0} d t \quad \therefore \boldsymbol{L} \omega_{0} \sin \theta=\frac{d \boldsymbol{L}}{d t}
$$

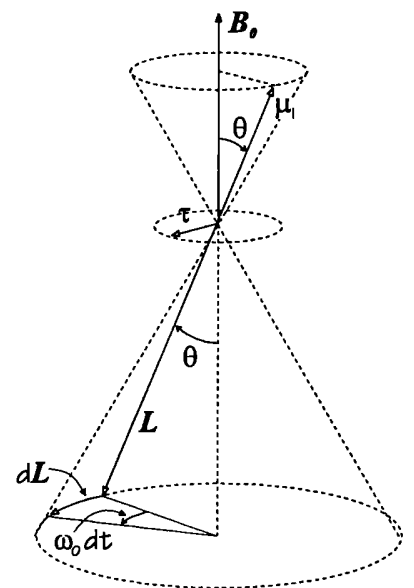

Figure 2.5- $A$ torque $\tau$ arises as the atom's magnetic dipole momentum $\mu_{l}$ interacts with the applied field $\boldsymbol{B}_{0 \text {. }}$ (from Eisberg, 1985)

and according to Newton's Law $(d \boldsymbol{L} / d t=\tau)$ and with equation (2.11) the angular frequency of precession is equal to,

$$
\omega_{0}=-\gamma B
$$

Equation (2.13) is known as the Larmor equation, and $\omega_{0}$ as the Larmor frequency. This is the fundamental equation of NMR and states that the nuclear magnetisation precession frequency is linearly proportional to the magnetic flux density $\boldsymbol{B}_{0}$.

The constant of proportionality is the gyromagnetic constant. For a magnetic field of 2.4 tesla, the hydrogen atom, (which has a gyromagnetic constant is equal to $\left.2.676 \times 10^{8} \mathrm{~s}^{-1} \mathrm{~T}^{-1}\right)$ will precess at a frequency of $100.00 \mathrm{MHz}$, for ${ }^{13} \mathrm{C}\left(\gamma=6.725 \times 10^{7}\right.$ $\left.\mathrm{s}^{-1} \mathrm{~T}^{-1}\right)$ the frequency is $15.35 \mathrm{MHz}$, and for ${ }^{23} \mathrm{Na}\left(\gamma=7.075 \times 10^{7} \mathrm{~s}^{-1} \mathrm{~T}^{-1}\right), 24.78 \mathrm{MHz}$ (Beal et al., 1984).

\subsubsection{Radiofrequency absorption and Macroscopic Magnetisation}

When the nucleus is placed into an external magnetic field $\boldsymbol{B}_{0}$ it will have the usual potential energy of orientation equal to $E=-\mu \cdot \boldsymbol{B}_{0}$ (Eisberg, 1895). Using 
equations (2.6) and (2.7), the potential energy of orientation is equal to,

$$
E=-\gamma \hbar m_{l} B_{0}
$$

and it can be seen that $m_{l}$ can have $(2 l+1)$ possible orientations relative to the externally applied field which corresponds to the same number of discrete energy levels for the nucleus.

For a nucleus with $l=1 / 2$, such as ${ }^{1} \mathrm{H}$, the angular momentum component in the $z$-direction can assume two states, where $m_{l}$ can be $-1 / 2$ and $+1 / 2$, figure 2.6 .

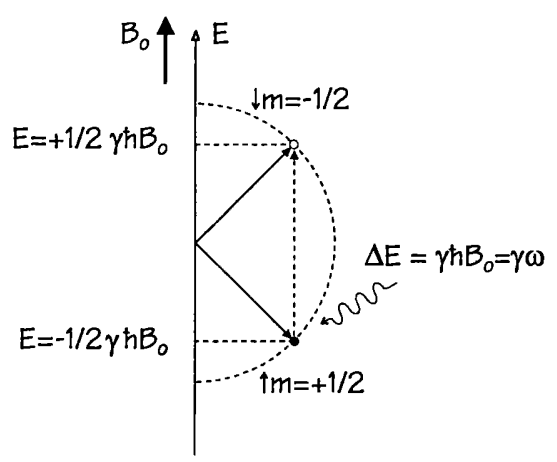

Figure 2.6- The energy-level diagram for a spin $1 / 2$ system. Transitions between the two states can be induced by electromagnetic energy of frequency $\omega=(\gamma / 2 \pi) B_{0}$.

From equation (2.14) the associated potential energy of orientation for each state in figure 2.6 is equal to,

$$
\begin{array}{ll}
E_{-\frac{1}{2}}=+\frac{\gamma \hbar B_{0}}{2} & m_{I}=-\frac{1}{2} \\
E_{+\frac{1}{2}}=-\frac{\gamma \hbar B_{0}}{2} & m_{I}=+\frac{1}{2}
\end{array}
$$

The lower energy state corresponds to the condition when the $z$-component of $\boldsymbol{L}$ is parallel to $\boldsymbol{B}_{0}$, while the higher energy state correspond to that in which $L_{z}$ is antiparallel to $\boldsymbol{B}_{\boldsymbol{0}}$. In the presence of an electromagnetic field oscillating at the correct frequency, stimulated absorption is possible, whereby a nuclear moment in the lower energy state is "flipped" into the higher state or the reverse process of stimulated emission may occur, whereby an excited nuclear moment returns to the lower energy state. In either case a quantum of energy equal to the difference in energy between the two states,

$$
\Delta E=\hbar \gamma B_{0}=\hbar \omega_{0}
$$

is absorbed or emitted respectively. $\omega_{0}$ is the angular frequency of the applied 
irradiation, which is equal to the Larmor frequency.

The next step is to determine the net spin magnetisation in the presence of an external magnetic field. In thermal equilibrium the average number of $+1 / 2$ spins $(n \uparrow)$ is given by the Boltzmann distribution, evaluated at the state energy $E \uparrow$,

$$
n \uparrow=n(E \uparrow)=A e^{-\left(\frac{E \uparrow}{k T}\right)}
$$

and similarly for $-1 / 2$ spins $(n \downarrow)$. Thus the population ratio has the value,

$$
\begin{aligned}
\frac{n \uparrow}{n \downarrow} & =e^{\frac{(-E \uparrow+E \downarrow)}{K T}} \quad \text { where } E \downarrow-E \uparrow=\Delta E=\gamma \hbar B_{0} \\
& =e^{-\left(\frac{\gamma \hbar B_{0}}{K T}\right)}
\end{aligned}
$$

where $k$ is Boltzman's constant $\left(k=1.38 \times 10^{-23} \mathrm{~J} / \mathrm{K}\right)$ and $T$ is the absolute temperature. The difference $n \uparrow-n \downarrow$ may be written as,

$$
n \uparrow-n \downarrow-n \frac{n \uparrow-n \downarrow}{n}=n \frac{n \uparrow-n \downarrow}{n \uparrow+n \downarrow}=n \frac{\frac{n \uparrow-n \downarrow}{n \uparrow}}{\frac{n \uparrow-n \downarrow}{n \uparrow}}=n \frac{1-\frac{n \downarrow}{n \uparrow}}{1-\frac{n \downarrow}{n \uparrow}}
$$

where $n=n \uparrow+n \downarrow$ is the total number of spins. Applying equation (2.18) and using the tanh function ${ }^{5}$,

$$
n \uparrow-n \downarrow=n \frac{1-e^{-2\left(\frac{\gamma \hbar B_{0}}{2 K T}\right)}}{1+e^{-2\left(\frac{\gamma \hbar B_{0}}{2 K T}\right)}}=n \tanh \left(\frac{\gamma \hbar B_{0}}{2 K T}\right)
$$

and since $\gamma \hbar B_{0} \ll K T$, the fraction excess of protons $(n \uparrow-n \downarrow) / n$ in the low state is equal to,

$$
\frac{n \uparrow-n \downarrow}{n} \approx \frac{\gamma \hbar B_{o}}{2 K T}
$$

From this it can be seen that at body temperature $(310 \mathrm{~K})$, in a magnetic field of $1 \mathrm{~T}$ (10,000 Gauss) the fraction of excess of protons in the low-energy state is only

5

$$
\tanh x=\frac{e^{x}-e^{-x}}{e^{x}+e^{-x}}=\frac{1-e^{-2 x}}{1+e^{-2 x}}
$$


$3.295 \times 10^{-6}$. This demonstrates why the NMR technique is inherently insensitive.

The slight excess of nuclear magnetic moments aligned along the direction of $\boldsymbol{B}_{\boldsymbol{o}}$ suggest that taking the vector sum of all such moment present in a particular sample will produce a net magnetization $\boldsymbol{M}_{\boldsymbol{o}}$. From equations (2.6) and (2.7), $\boldsymbol{M}_{\boldsymbol{o}}$ may approximate to (Morris, 1986),

$$
M_{o} \approx(n \uparrow-n \downarrow) \mu \approx(n \uparrow-n \downarrow) \gamma m_{l} \hbar
$$

substituting equation (2.21) into (2.22),

$$
\boldsymbol{M}_{\boldsymbol{o}} \approx \frac{\gamma^{2} \hbar m_{l} n}{2 k T} \boldsymbol{B}_{\boldsymbol{o}}
$$

for the case of the hydrogen nucleus, $m_{l}=1 / 2$ and the net magnetisation is equal to

$$
M_{o} \approx \frac{\gamma^{2} \hbar n}{4 k T} B_{o}
$$

and the magnetic susceptibility $\chi_{\mathrm{m}}$ is,

$$
\chi_{m}=\frac{M_{o}}{B_{o}} \approx \frac{\gamma^{2} \hbar n}{4 k T}
$$

Equation (2.24) implies that an improvement in sensitivity may be obtained by either decreasing the temperature, obviously difficult to achieve in biological specimens, or increasing $\boldsymbol{B}_{0}$.

\subsubsection{The rotating frame concept}

The nuclear spins precess about the magnetic field $\boldsymbol{B}_{\boldsymbol{0}}$, with a frequency equal to the Larmor frequency. In order to visualise the behaviour of the precessing magnetisation, it is helpful to introduce a coordinate system $\left(x^{\prime}, y^{\prime}, z^{\prime}\right)$ rotating at the same frequency $\omega_{0}$ as the Larmor frequency. The $z^{\prime}$-axis is usually defined coincident with the $z$-axis of the fixed laboratory frame $(x, y, z)$.

In the rotating frame the individual nuclear magnetic moments appear as stationary vectors, figure $2.7(\mathrm{a})$, and the net magnetisation $\boldsymbol{M}_{0}$, given by equation (2.24) is in the same direction as $z^{\prime}$, and rotates about the laboratory frame axis $z$.

To analyse the effects of a rotating frame, consider the magnetisation $\boldsymbol{M}$ given by,

$$
M=M_{x}^{\prime} \hat{\boldsymbol{a}}_{x}+M_{y}^{\prime} \hat{\boldsymbol{a}}_{y}+M_{z}^{\prime} \hat{\boldsymbol{a}}_{z}
$$

where $\hat{a}_{x}, \hat{a}_{y}$ and $\hat{a}_{z}$ are unit vectors along the $x^{\prime}, y^{\prime}$ and $z^{\prime}$ axes of the rotating frame, 


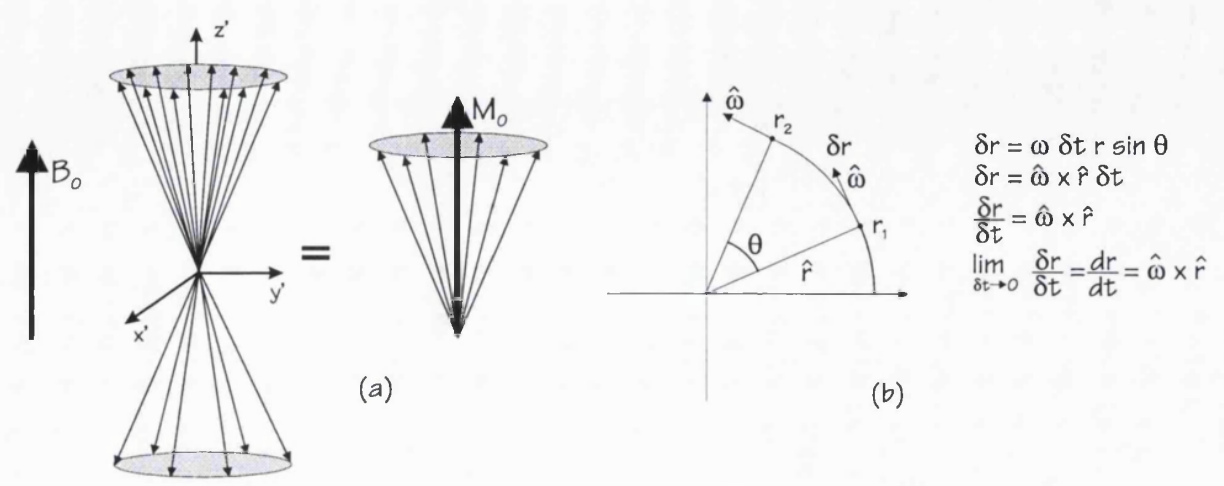

Figure 2.7 - The distribution of individual nuclear magnetic moments in the rotating frame for a hydrogen atom, spin $1 / 2$.

and $M_{x}^{\prime}, M_{y}^{\prime}$ and $M_{z}^{\prime}$ are the corresponding components of magnetisation along these directions. Then, for a rotating frame with an angular velocity $\omega$, (figure $2.7(\mathrm{~b})$ ),

$$
\frac{\partial \hat{a}_{x}}{\partial t}=\omega \times \hat{a}_{x} \quad \frac{\partial \hat{a}_{y}}{\partial t}=\omega \times \hat{a}_{y} \quad \frac{\partial \hat{a}_{z}}{\partial t}=\omega \times \hat{\boldsymbol{a}}_{z}
$$

and the differential of $\boldsymbol{M}$ with respect to the time will be

$$
\begin{aligned}
& \frac{d \boldsymbol{M}}{d t}=\left(\hat{\boldsymbol{a}}_{x} \frac{\partial M_{x}^{\prime}}{\partial t}+\hat{\boldsymbol{a}}_{y} \frac{\partial M_{y}^{\prime}}{\partial t}+\hat{\boldsymbol{a}}_{z} \frac{\partial M_{z}^{\prime}}{\partial t}\right)+\left(M_{x}^{\prime} \frac{\partial \hat{\boldsymbol{a}}_{x}}{\partial t}+M_{y}^{\prime} \frac{\partial \hat{\boldsymbol{a}}_{y}}{\partial t}+M_{z}^{\prime} \frac{\partial \hat{\boldsymbol{a}}_{z}}{\partial t}\right) \\
& \frac{d \boldsymbol{M}}{d t}=\left(\frac{\partial \boldsymbol{M}}{\partial t}\right)_{\text {totat }}+(\omega \times \boldsymbol{M})
\end{aligned}
$$

Now, from equation (2.11), $\tau=\gamma \boldsymbol{L} \times \boldsymbol{B}$, and from Newton's Law, $d \boldsymbol{L} / d t=\tau$, hence $d \boldsymbol{L} / d t=\gamma \boldsymbol{L} \times \boldsymbol{B}_{0}$ and changing $\boldsymbol{L}$ for $\boldsymbol{M}$,

$$
\frac{d \boldsymbol{M}}{d t}=\gamma \boldsymbol{M} \times \boldsymbol{B}
$$

combining equation (2.28) into (2.29),

$$
\left(\frac{\partial \boldsymbol{M}}{\partial t}\right)_{\text {rotat }}+(\omega \times \boldsymbol{M})=\gamma(\boldsymbol{M} \times \boldsymbol{B})
$$

thus the motion of the magnetic moment in the rotating frame is given by,

$$
\begin{aligned}
& \left(\frac{\partial \boldsymbol{M}}{\partial t}\right)_{\text {rotat }}=\gamma\left(\boldsymbol{M} \times \boldsymbol{B}_{0}\right)-\omega \times \boldsymbol{M}=\boldsymbol{M} \times\left(\gamma \boldsymbol{B}_{0}+\omega\right) \\
& \left(\frac{\delta \boldsymbol{M}}{\delta t}\right)_{\text {rotut }}=\gamma \boldsymbol{M} \times\left(\boldsymbol{B}_{0}+\frac{\omega}{\gamma}\right)
\end{aligned}
$$


Hence in the rotating frame, $\boldsymbol{M}$ follows the same equation of motion as in the laboratory frame but with an effective field $\boldsymbol{B}_{\text {eff }}$ equal to

$$
\boldsymbol{B}_{\text {eff }}=\boldsymbol{B}_{0}+\frac{\omega}{\gamma}
$$

or one could say that the magnetisation $\boldsymbol{M}$, precess about $\boldsymbol{B}_{\text {eff }}$ when viewed in the rotating frame. By choosing a rotating frame with $\omega=-\gamma \boldsymbol{B}$, the effective field $\boldsymbol{B}_{\text {eff }}$ vanishes. In this frame $\partial M / \partial t=0$ and the magnetic moment is a fixed vector, figure 2.8 .

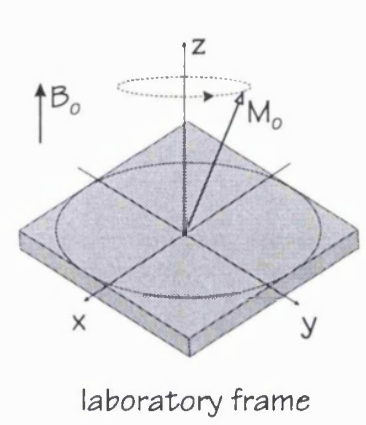

(a)

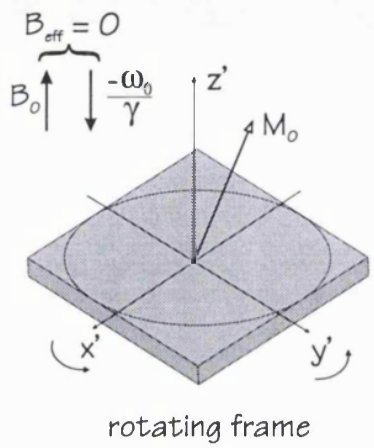

(b)

Figure 2.8-(a) the magnetization $\boldsymbol{M}$ precessing around $\boldsymbol{B}_{\|}$in the laboratory frame, (b) the magnetisation is static in the rotating frame since $\boldsymbol{B}_{\text {eff }}=0$.

\subsubsection{The RF Field $B_{1}$}

Consider that a circularly polarized RF field of frequency $\omega$ (in the laboratory frame) and with magnetic flux density $\boldsymbol{B}_{l}$, is applied to the spin magnetisation system in a plane perpendicular to $\boldsymbol{B}_{0}{ }^{6}$. The effective flux density in the rotating frame will be,

${ }^{6}$ For technical reasons in most NMR equipment, a plane-polarized RF field is applied. A plane-polarised field with flux density $2 \boldsymbol{B}_{\boldsymbol{l}} \cos (\omega t)$ can be considered as the superposition of two circularly polarised fields with flux density $\boldsymbol{B}_{\boldsymbol{l}}$ rotating in opposite direction, figure $\mathrm{A}$.

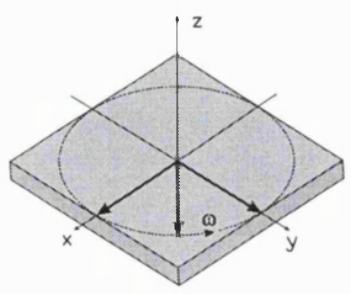

$B 1\left(\cos \omega_{0} t, \sin \omega_{0} t, 0\right)$

(a)

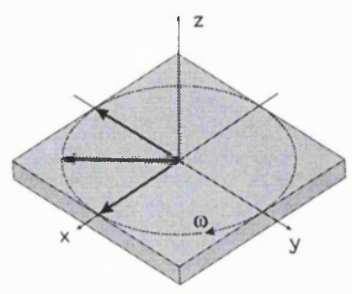

$B 1\left(\cos \omega_{0} t, \sin -\omega_{0} t, O\right)$

(b)

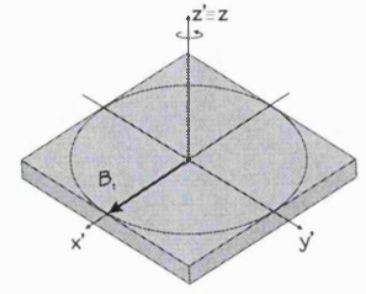

$B 1\left(\cos \omega_{0} t, 0,0\right)$

(c)

Figure A-The two counter-rotating components $(a)$ and (b), and the resultant linearly-polarized field. The field rotating in the same sense as the precession will interact with $\boldsymbol{M}_{\boldsymbol{j}}$, whilst the counter-rotating field will have negligible effect. The linear or plane-polarised field is, however, less efficient, as power deposited is twice that required (Webb, 1995). 


$$
\boldsymbol{B}_{\text {eff }}=\left(\boldsymbol{B}_{0}+\frac{\omega}{\gamma}\right) \hat{a}_{z}+B_{1} \hat{a}_{x}
$$

if $\omega=\omega_{0}, \boldsymbol{B}_{\text {eff }}=\boldsymbol{B}_{I}$ and $\boldsymbol{B}_{I}$ appears stationary in the rotating frame, being the only component of $\boldsymbol{B}_{\text {eff }}$. Thus it follows from section 2.1.2, that the net magnetization $\boldsymbol{M}_{0}$ will precess about the $\boldsymbol{B}_{I}$ field with an angular frequency equal to $\boldsymbol{\gamma} \boldsymbol{B}_{I}$, figure 2.9.

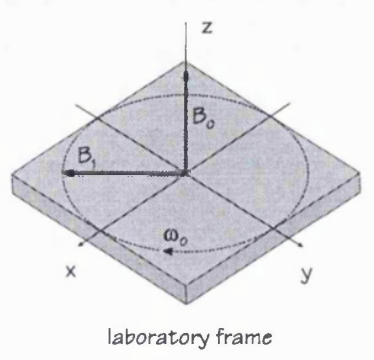

(a)

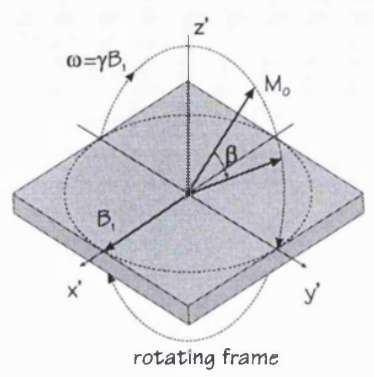

(b)

Figure 2.9- (a) The rotating magnetic fields in the laboratory frame. (b) The rotation of $\boldsymbol{M}_{0}$ about $\boldsymbol{B}_{I}$ in the rotating frame.

Since $\boldsymbol{B}_{\boldsymbol{I}}$ is applied for a short period of time it rotates $\boldsymbol{M}_{0}$ (given by equation (2.24)) by an angle $\beta$ equal to,

$$
\beta=\gamma\left|B_{1}\right| t_{p}
$$

where $t_{p}$ is the time for which $\boldsymbol{B}_{\boldsymbol{l}}$ is applied, figure 2.9(b).

Since NMR resonance frequencies are typically in the megahertz region and $t_{p}$ is typically $1 \mu \mathrm{s}-100 \mu \mathrm{s}$, the $\boldsymbol{B}_{1}$ field is often referred to as the RF pulse.

Therefore, prior to the RF pulse, the spins rotate about $\boldsymbol{B}_{0}$ which is aligned along the $z$-axis. At this point, there is no net-magnetisation in any direction within the $x y$ plane. The RF pulse then tips the net magnetisation away from the $z$-axis, towards the $x y$-plane of the rotating frame.

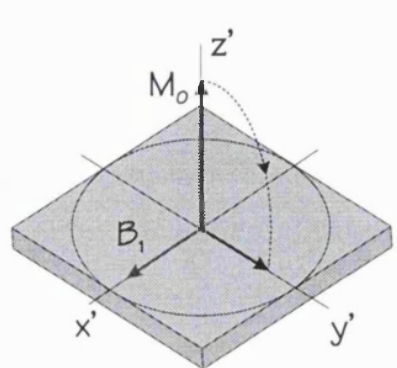

(a)
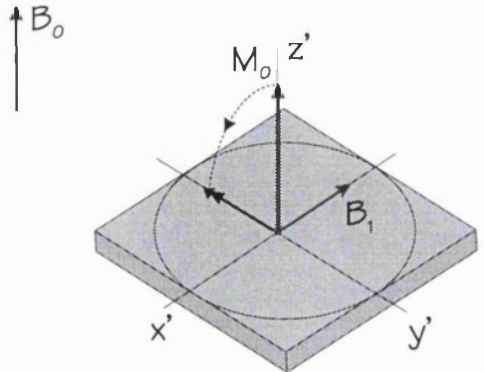

(b)

Figure 2.10- The net magnetisation rotation. (a) a $+90^{\circ} B, R F(b)-90^{\circ}$ RF pulse.

If in equation (2.34) $\beta=90^{\circ}$ and $\boldsymbol{B}_{\boldsymbol{l}}$ is applied along $+x$-axis, the net 
magnetisation rotates towards $+y$ and one talks about a $+90^{\circ}$ pulse, (figure 2.10(a)). If $B_{1}$ is applied in the $-x$ direction the net magnetisation rotates toward $-y$ and one talks about a $-90^{\circ}$ pulse, figure $2.10(\mathrm{~b})$.

\subsubsection{The Relaxation Phenomena and Bloch's equations}

Spin-lattice relaxation $\left(T_{1}\right)$

Spin-lattice relaxation also called longitudinal relaxation time is the way by which the spin population after being tipped by a $90^{\circ}$ pulse gradually returns to its equilibrium Boltzmann distribution along the $+z$ direction. This process is illustrated in figure 2.11 .

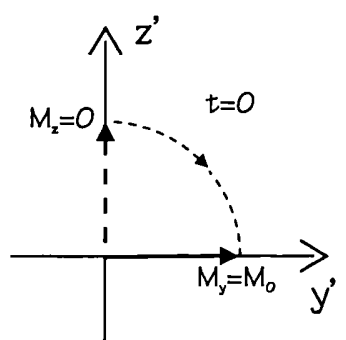

(a)

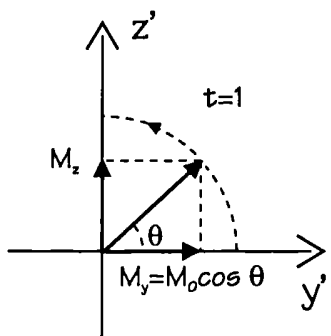

(b)

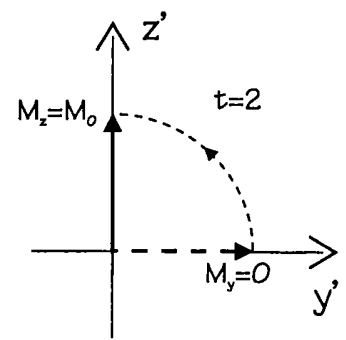

(c)

Figure 2.11-Spin-lattice relaxation time $T_{1}$ viewed from the rotating frame. After the $90^{\circ}$ pulse (a), the net magnetisation gradually returns to the original distribution $(c)$.

Following the application of $\boldsymbol{B}_{\boldsymbol{1}}$ for a time $t_{p}$, the RF energy absorption tip the spins into the $x y$-plane. Once the $\boldsymbol{B}_{1}$ field is removed, the energy exchange of the spins with their surrounding (the "lattice" of which the nuclear spin are part) make them return to the direction of $\boldsymbol{B}_{0}$. The trend of the magnetisation towards its equilibrium $M_{z}=M_{0}=\chi_{0} B_{0}$ can be described by the equation,

$$
\frac{d M_{z}}{d t}=-\frac{\left(M_{z}-M_{0}\right)}{T_{1}}
$$

and the magnetisation recovery towards the equilibrium value $\boldsymbol{M}_{\boldsymbol{0}}$ is exponential.

Generally in an NMR experiment a sequence of RF pulses are applied to the sample in a interval called the repetition time $T R$. If $T R$ is equal to, or greater than $5 T_{1}$, the magnetisation in the $x^{\prime} y^{\prime}$-plane is equal to $\boldsymbol{M}_{0}$, since enough time for a full relaxation has elapsed. However, if $T R$ is comparable to $T_{1}$, the system will not have enough time to recover along $z^{\prime}$ - before the net magnetisation is exposed to a new $90^{\circ}$ pulse. This 
next $90^{\circ}$ rotation is then added to the remaining angle between $\boldsymbol{M}_{\boldsymbol{0}}$ and $\boldsymbol{B}_{\boldsymbol{0}}$ bringing the net magnetisation out of the $x^{\prime} y^{\prime}$-plane, and the observable net magnetisation, (the observable magnetisation is just the component of $\boldsymbol{M}_{0}$ parallel to the $x^{\prime} y^{\prime}$-plane), will be smaller than $\boldsymbol{M}_{\boldsymbol{0}}$. In the simplest case, the return to equilibrium is a monoexponetial function,

$$
M_{z}=M_{z}(0)\left(1-e^{-\frac{T R}{T_{1}}}\right)
$$

Therefore, by varying $T R$ it is possible to reduce the signal emerging from specific parts of a sample where $T_{1}$ is different. Also, different samples respond very differently to a train of equidistant $90^{\circ}$ pulses. This is the basis for $T R$-influenced contrast behaviour in MR imaging.

For liquid systems such as water, typical $T_{1}$ relaxation times lie in the range 0.1 $10 \mathrm{~s}$. In solids, where the motion is considerably less, $T_{l}$, relaxation times of minutes or hours are not uncommon.

\section{Spin-spin relaxation time $\left(T_{2}\right)$}

After the spin system is tipped into the in the $x^{\prime} y^{\prime}$ - plane the microscopic components of the macromagnetisation tend to become disordered, losing their initial "phase coherence" and hence, destroying the net magnetisation along the $x^{\prime} y^{\prime}$-plane. As illustrated in figure 2.12 just after the $90^{\circ} \mathrm{RF}$ pulse the net magnetisation lies in the $y^{\prime}$ direction and starts to dephase until all spins are completely spread out into $x y$-plane and the net magnetisation is reduced to zero. This process is characterised by $T_{2}$, the spinspin or transverse relaxation and it is much faster than the relaxation time $T_{1}$.

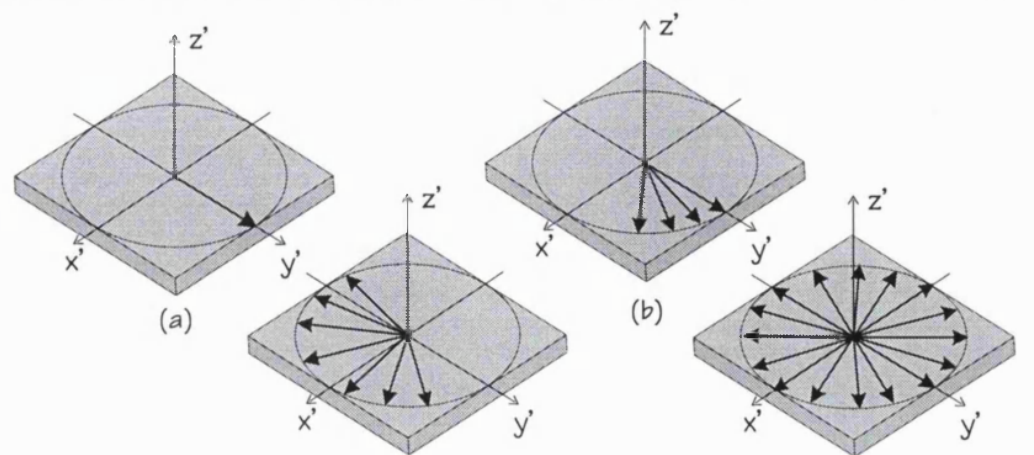

(c)

(d)

Figure 2.12-Spin-spin relaxation time $T_{2}$ viewed from the rotating frame.

Two processes are involved on this phenomena: natural and local field inhomogeneities. In the natural dephasing process, one of the principal contributions is 
the dipole-dipole interaction (Morris, 1986). Each nuclear spin produces its own magnetic field. The interaction among these individual magnetic fields produces a locally slightly different Larmor frequency, dephasing the components of the net magnetisation in the $x^{\prime} y^{\prime}$-plane. The transverse magnetisation decays at a rate represented by the equation

$$
\frac{d M_{x}^{\prime}}{d t}=-\frac{M_{x}^{\prime}}{T_{2}} \quad \frac{d M_{y}^{\prime}}{d t}=-\frac{M_{y}^{\prime}}{T_{2}}
$$

$T_{2}$ is dependent on a number of parameters such as, observation frequency, temperature, mobility of the observed spin (microviscosity) and presence of large molecules, paramagnetic ions and molecules, or other outside interference. In mobile fluids $T_{2}$ is nearly equal to $T_{1}$, whereas in solids or high-viscosity systems, static field components induced by neighbouring nuclei are operative and $T_{2}$ becomes significantly shorter than $T_{l}$. To a large extent, this is the cause of the low or absent signal from solid structures such as bone or tendons in medical magnetic resonance imaging (Rinck, 1993).

Two phenomena contribute to the local field imperfections experienced by the nuclei: static and oscillating fields locally induced by neighbouring magnetic moments (from other nuclei or unpaired electrons), and the imperfections in the externally applied uniform magnetic field. Both phenomena give rise to local variations in the Larmor frequency. Therefore, a group of spins initially in phase will therefore gradually accumulate a phase error depending on the local field each spin experiences. This leads to a decay of the observed signal which is faster than $T_{2}$. The observable decay is denoted by $T_{2}^{*}$ and is given by,

$$
\frac{1}{T_{2}^{*}}=\frac{1}{T_{2}}+\frac{\gamma \Delta B_{0}}{2}
$$

where $\Delta \mathrm{B}_{0}$ is the absolute value of the field inhomogeneity experienced by the spins.

Equations (2.35) and (2.37) are the phenomenological Bloch equations proposed by Felix Bloch in 1946 for the description of magnetic properties of ensembles of nuclei in an external magnetic field in the absence of any RF driving field. Combining equations (2.29), (2.35) and (2.37) a general equation describing the evolution of the 
motion for $\boldsymbol{M}_{\boldsymbol{0}}$ in the rotating frame may be written (Abragam, 1961),

$$
\frac{d \boldsymbol{M}_{0}}{d t}=\gamma \boldsymbol{M}_{0} \times \boldsymbol{B}-\frac{\left(M_{x} \hat{a}_{x}+M_{y} \hat{a}_{y}\right)}{T_{2}}-\frac{\left(M_{z}-M_{0}\right)}{T_{1}} \hat{a}_{z}
$$

\subsubsection{The free induction decay and spin echo signals}

In a typical NMR experiment, to apply the RF pulse and to detect the signal induced by the net rotating magnetisation, a single coil (inductor) is used orientated at right angles to the main magnetic field. The NMR sample is placed at the centre of the coil making it part of the tuned circuit resonant at the Larmor frequency $\omega_{0}$.

After the application of the $90^{\circ} \mathrm{RF}$ pulse, the net magnetisation is tipped into the plane of the coil, figure 2.12(a). The individual spins start to lose phase coherence in the $x^{\prime} y^{\prime}$-plane figure 2.12(b), i.e. they separate from each other and fan out. Hence the net magnetisation and the NMR signal $S(t)$ induced in the coil reduces until eventually no magnetisation exists. In the simplest case the signal detected may be decomposed into two components, one sinusoidal and related to the constant angular velocity about the $z$-axis, and another exponential, related to the spin-spin relaxation $T_{2}$,

$$
S(t)=k M_{0} \sin \left(\omega_{0}+\varphi\right) e^{\left(-\frac{t}{T_{2}}\right)}
$$

where $\mathrm{k}$ and $\varphi$ are constants dependent upon the nature of the receivers coil, etc ... This signal is referred to as the free induction decay or FID.

As explained before, two process are involved with the dephasing: one due to the spin-spin relaxation $T_{2}$, which is irreversible, since it is a quantum incoherent process and, another due to field imperfections $T_{2}{ }^{*}$ which is a reversible process. To recover the signal, after a time delay $\tau$ figure $2.13(\mathrm{c})$, the system is exposed to a $180^{\circ}$ pulse, and a refocusing process is initialised. The $180^{\circ}$ pulse changes the phase of each spin by $180^{\circ}$; that is, it reverses its phase into the $-y^{\prime}$-axis. They will continue to rotate in the same direction, but now the spins precessing with a frequency slightly in excess of $\omega_{0}$ lag those precessing at the Larmor frequency, and those precessing with a lower frequency lead those precessing at the Larmor frequency. After a time $\tau$ all the spins are in phase, and a large signal is induced figure 2.13(d), following which the spins start to dephase and reduce the signal again, figure 2.13(e). 


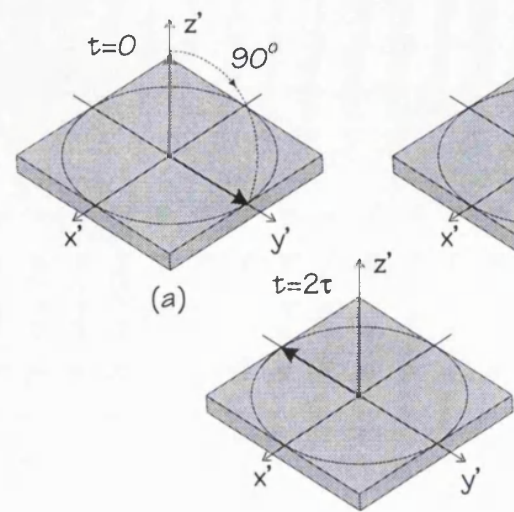

(d)

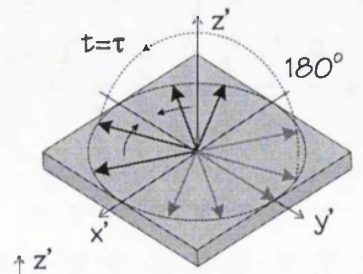

(c) (b)

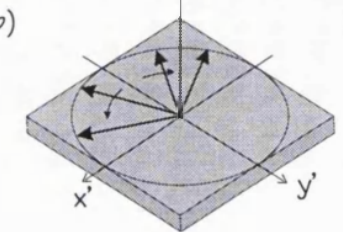

(e)

Figure 2.13- The $180^{\circ}$ refocusing pulse

This $90^{\circ}-180^{\circ} \mathrm{RF}$ pulse sequence is called a spin-echo $(S E)$, and as a result, after a time equal to $2 \tau$ an echo signal is induced in the coil. The $2 \tau$ interval is called the echo time (TE). By repeating the $180^{\circ}$ pulse again, further echoes of decreasing amplitude are created. This is described as multiple spin echo or multi-echo sequence. In figure 2.14 the evolution of the magnetisation in the $x^{\prime} y^{\prime}$-plane of the rotating frame is illustrated, with the $T_{2}$ and $T_{2}{ }^{*}$ relaxation times.
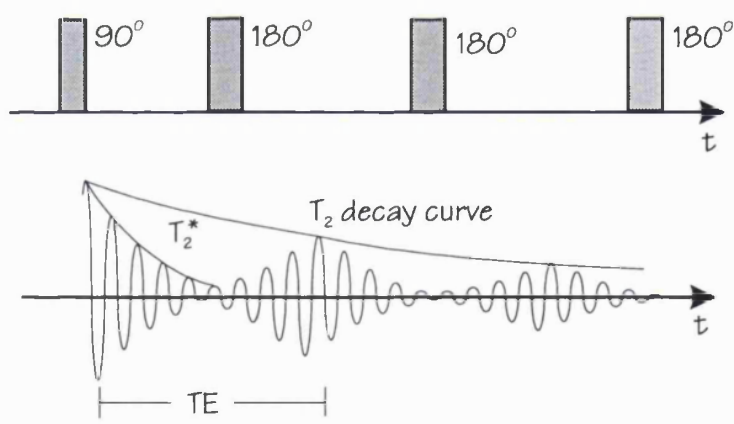

Figure 2.14- Signal evolution $M_{x y} /$ in the spin echo-time sequence pulse. Upper: The $90^{\circ}$ and $180^{\circ} R F$ pulses. Lower: The signal evolution.

\subsection{Imaging principles}

The formation of an image involves the following procedures:

(1) localisation and excitation of the spins of interest;

(2) the spatial encoding of their signal; and

(3) the signal detection and reconstruction.

\subsubsection{The slice selection}

In NMR experiments a sample is placed in a magnetic field $\boldsymbol{B}_{0}$ which is shimmed 
to make it as uniform as possible. Since the angular frequency of precession is proportional to the main magnetic field $\boldsymbol{B}_{0}$, equation (2.13), all spins in the sample are subject to the same magnetic field therefore will absorb at the same frequency. This set of spins is referred to as a spin isochromat, as they give rise to an unique frequency component (sine/cosine).

Consider now the presence of a gradient magnetic field $G_{z}$ parallel to the main field $\boldsymbol{B}_{0}$, as illustrated in figure 2.15 . Whereby each region of an object experiences a total magnetic field given by,

$$
\boldsymbol{B}_{\text {total }}=\left(\boldsymbol{B}_{0}+z G_{z}\right)
$$

and thus precesses with a particular angular frequency equal to,

$$
\omega(z)=\gamma\left(\boldsymbol{B}_{0}+z G_{z}\right)
$$

Applying a $90^{\circ} \mathrm{RF}$ pulse, with a bandwidth equal to $\Delta \omega$, only those spins with an angular frequency of precession that match $\Delta \omega$ are tipped into the plane coil and contribute to the NMR spin-echo signal.

(a)

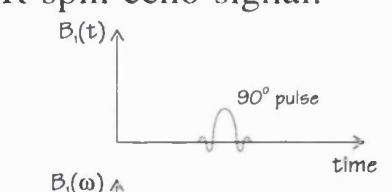

(b)

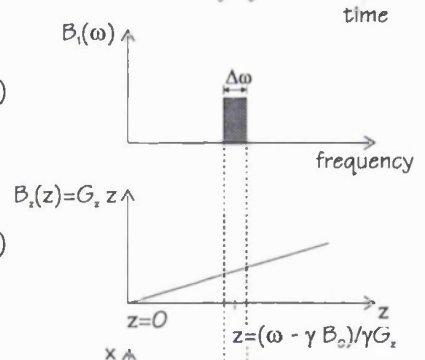

(d)

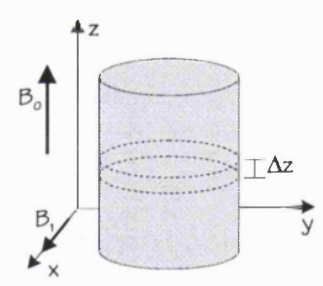

(e)

Figure 2.15- Illustration of the process to selected a tomographic plane.

In the presence of a linear gradient field $G_{z}$, figure 2.15(c), the spins which are excited by the $90^{\circ} \mathrm{RF}$ pulse, figure $2.15(\mathrm{~b})$, lie in a plane of thickness $\Delta z$, figure $2.15(\mathrm{e})$, given by,

$$
\Delta \omega=\gamma \Delta B=\gamma G_{z} \Delta z \quad \therefore \quad \Delta z=\frac{\Delta \omega}{\gamma G_{z}}
$$

normal to the gradient direction, figure 2.15(d), and centred at, 


$$
z=\frac{\omega-\gamma B_{0}}{\gamma G_{z}}
$$

The tomographic plane orientation may be adjusted by modifying the orientation of the magnetic field gradient before the $90^{\circ} \mathrm{RF}$ pulse, and the slice thickness by changing the gradient field or RF pulse bandwidth.

This is the first step in the process of NMR imaging, whereby only those spins in a specific plane through the object are excited and contribute to the NMR signal induced into the coil.

\subsubsection{Spatial encoding}

Once the spins of a particular region or slice have been excited they are rotating into the image coil plane and the NMR signal can be detected. But before it is detected, it is necessary to spatially encode the signal to obtain information about the spin positions in the plane. The spatial encoding process is divided into two methods: frequency encoding and phase encoding.

\section{- The frequency encoding principle}

As described above, the association of a gradient magnetic field $G_{z}$ with the $90^{\circ}$ $\mathrm{RF}$ pulse makes it possible to select just the spins of a tomographic plane (parallel to the $x y$-plane) through the object being imaged.

Consider $\rho(x, y)$ the two-dimensional spin density of the cylinder slice selected in figure 2.15, divided into a matrix of $n \times n$ image elements or pixels as shown in figure 2.16 .

(a)

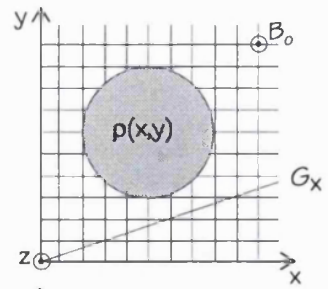

(b)

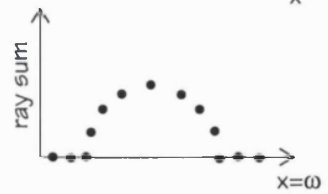

Figure 2.16-(a) Spin density of the slice selected in figure 2.15.(b) The resulting projection of solving the integral of equation (2.47) into the y-direction.

If a gradient field is placed in the $x$-direction, all the spins lying on a particular line perpendicular to the $G_{x}$ gradient direction will experience a total magnetic field 
equal to $\boldsymbol{B}_{\boldsymbol{x}}=\boldsymbol{B}_{0}+x G_{x}$, and will precess with an angular frequency of,

$$
\omega(x)=-\gamma\left(\boldsymbol{B}_{0}+x G_{x}\right)
$$

The NMR signal is then demodulated, to remove the main frequency $\omega_{0}\left(=\gamma \boldsymbol{B}_{0}\right)$, leaving the angular frequency offset $\Delta \omega(x)=\gamma x G_{x}$. From equation (2.59), using the Euler identity, not considering the phase $\varphi$ and replacing $\boldsymbol{M}_{\boldsymbol{0}}$ by $\rho(x, y)$, the NMR signal of a single spin isochromat is therefore equal to,

$$
d S(t)=k \rho(x, y) e^{i\left(\gamma \times G_{x} t\right)} d x d y
$$

and the total signal from the whole slice is given by,

$$
S(t)=k \int_{x} \int_{y} \rho(x, y) e^{i\left(\gamma x G_{x} t\right)} d x d y
$$

In equation (2.47), the integral in the $y$-direction is the single frequency "ray sum", which is shown in figure 2.16(b). The resulting function of the $y$-integration is the NMR spectrum obtained by Fourier transforming the spin-echo signal recorded in the presence of gradient $G_{x}$. The $G_{x}$ gradient is also called read out gradient.

\section{The phase encoding principle}

The phase encoding principle consists of applying a further gradient field in the $y$-direction before the NMR signal is acquired in the presence of the reading gradient. Hence, additional information about the second dimension $y$ is incorporated in equation (2.47).

In figure $2.17(\mathrm{a})$, following the $90^{\circ} \mathrm{RF}$ pulse the $G_{x}$ gradient is applied and the FID signal acquired. Ignoring the relaxation time and after demodulation, the signal detected is that given by equation (2.47). In figure 2.17(b) is illustrated the so called zeugmatography method. Here, following the $90^{\circ} \mathrm{RF}$ pulse a gradient is applied in the $y$-direction and following that the $G_{x}$ gradient is applied for the signal acquisition. The effect of $G_{y}$ is to alter the frequency of precession of the spins and therefore change the phase of the FID signal. The equivalent equation (2.47) for the zeugmatography method can be obtained by adding the $G_{y}$ gradient to it,

$$
S\left(t_{x}, t_{y}\right)=\int_{x} \int_{y} \rho(x, y) e^{i \gamma\left[x G_{x} t_{x}+y G_{y} t_{y}\right]} d x d y
$$




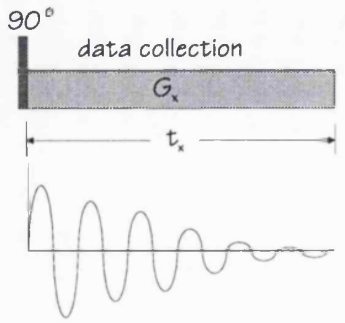

(a)

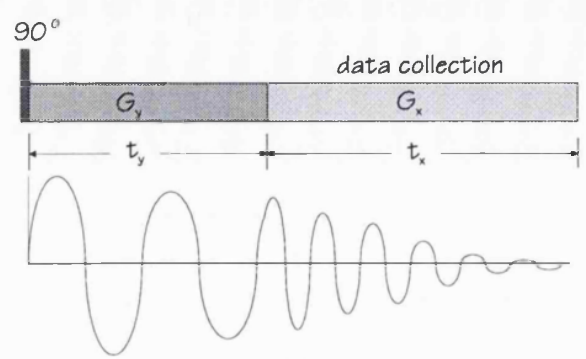

(b)

Figure 2.17- The phase encoding principle. (a) The frequency encoding; (b) The phase encoding followed by the frequency encoding (from Morris, 1986).

The two-dimensional Fourier transform $\mathrm{F}\left(k_{x}, k_{y}\right)$ of the spin density $\rho(x, y)$, where $k_{x}$ and $k_{y}$ are the wavenumbers in the $x$ - and $y$-direction respectively, is defined as,

$$
F\left(k_{x}, k_{y}\right)=\int_{-\infty}^{\infty} \int_{-\infty}^{\infty} \rho(x, y) e^{-i\left(x k_{x}+y k_{y}\right)} d x d y
$$

Comparing equations (2.48) and (2.49) it is possible to see that $S\left(t_{x}, t_{y}\right)=F\left(k_{x}, k_{y}\right)$, which implies (Ljunggren, 1983),

$$
\begin{aligned}
& k_{y}=\gamma G_{y} t_{y}=\omega_{y} t_{y} \\
& k_{x}=\gamma G_{x} t_{x}=\omega_{x} t_{x}
\end{aligned}
$$

$K_{x}$ and $K_{y}$ defines the so called $k$-space which allows one to visualise the scan trajectory during the NMR data acquisition.

Thus by acquiring a data set with different $t_{v}$ an $n \times n$ matrix of $S\left(t_{x}, t_{y}\right)$ is obtained,

$$
\left[\begin{array}{cccc}
S\left(\tau, t_{0}\right) & S\left(2 \tau, t_{0}\right) & \ldots & S\left(n \tau, t_{0}\right) \\
S\left(\tau, t_{1}\right) & S\left(2 \tau, t_{1}\right) & \ldots & S\left(n \tau, t_{1}\right) \\
\cdot & \cdot & \ldots & \cdot \\
\cdot & \cdot & \ldots & \cdot \\
\cdot & \cdot & \ldots & \cdot \\
S\left(\tau, t_{n}\right) & S\left(2 \tau, t_{n}\right) & \ldots & S\left(n \tau, t_{n}\right)
\end{array}\right]
$$

In the matrix each line corresponds to a complete FID which is sampled in a regular interval time $\tau$. The number of samples determines the number of pixels and the resolution in the $x$-direction and the number of FID's collected determines the numbers of pixels and the resolution in $y$-direction. For each FID signal acquired, a different $t_{v}$ 
gradient time is used, hence each FID acquired represents a different image projection since the FID phase is different.

After all the data has been acquired the image reconstruction process can start. First the Fourier transform is applied in the $x$-direction (matrix line), then it is applied in the $y$-direction (matrix column). The Fourier component in the $y$-direction is better understood if a single point $\left(x_{0}, y_{0}\right)$ is considered. Again, ignoring the relaxation phenomena, the FID signal induced in the coil by the set of spins at this point would be a unique harmonic (sine or cosine). In the presence of $G_{y}$, the frequency of the FID is equal to,

$$
\omega\left(x_{0}, y_{0}\right)=\gamma y_{0} G_{y}
$$

and in presence of the following $G_{x}$ gradient, during the FID acquisition, it is equal to,

$$
\omega\left(x_{0}, y_{0}\right)=\gamma x_{0} G_{x}
$$

Since each matrix line is acquired with different $t_{y}$, a proportional phase shift is introduced in each FID signal acquired. The effect of this phase shift increment, is an amplitude modulation of the Fourier transform components, in this case (sine/cosine) equivalent to an impulse, or $\delta$-function, in the frequency domain (Brigham, 1974). Figure 2.18 illustrates the phase shift and the $\delta$-function modulation.

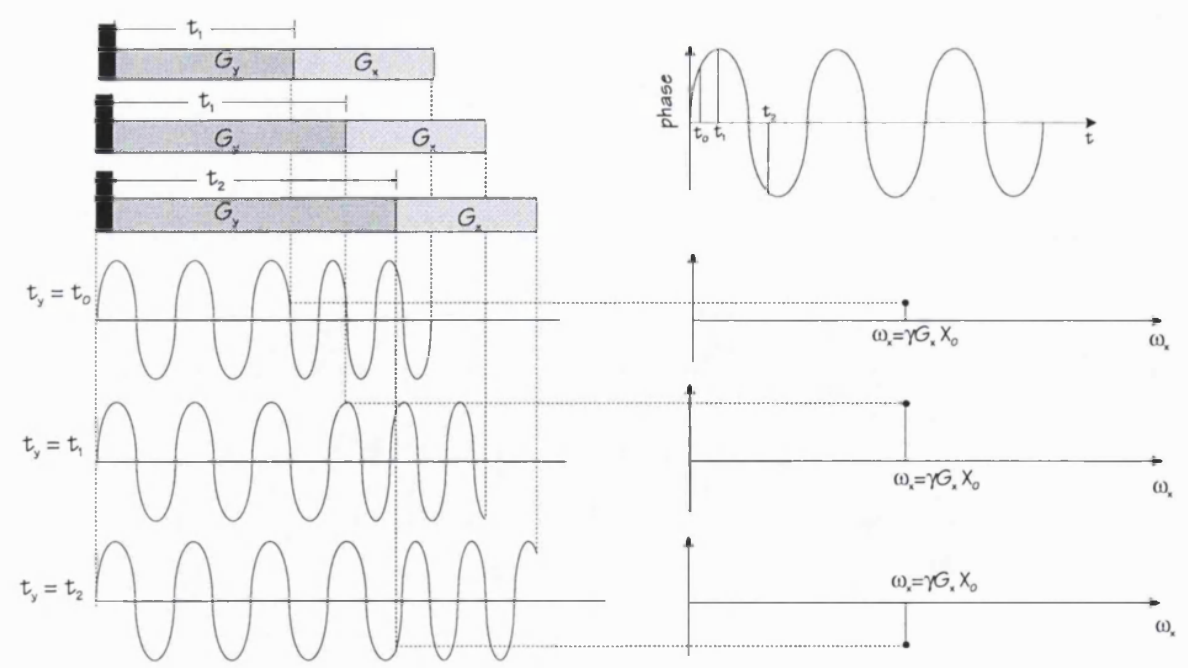

Figure 2.18 - The phase encoding modulation on the FID collection.

If the amplitude of each $\delta$-function is plotted in the time domain the result will be a sine (or cosine) function with a frequency equal to that of equation (2.51). Thus, when performing the Fourier transform in this matrix column all pixels in the $y$-direction 
are set to zero apart from that at $\left(x_{0}, y_{0}\right)$.

Therefore, the two-dimensional Fourier transformed matrix consist of a single point at frequency co-ordinates corresponding to $\left(\mathrm{x}_{0}, \mathrm{y}_{0}\right)$ with a magnitude proportional to the spin density $\rho\left(x_{0}, y_{0}\right)$ at that point (Morris, 1986).

\subsubsection{The spin-echo imaging sequence}

The process of phase encoding in the zeugmatographic Fourier technique has the disadvantage that the interval between successive FID's is increased. This leads to a degradation of the FID signal caused by the relaxation process $\mathrm{T}_{2}{ }^{*}$. Besides, with the implementation of gradients we can locate the nuclei in our sample but when the gradient is switched on the gradient significantly reduces the magnitude of the magnetic resonance signal from the sample.

A sequence that overcomes this disadvantage is the spin-echo imaging experiment illustrated in figure 2.19. Here instead of acquire the FID signals after the $90^{\circ} \mathrm{RF}$ pulse and phase encoding, the spin-echo sequence (item 2.1.7) is used and the actual signal acquired is the echo signal produced after the refocusing $180^{\circ}$ pulse. In this experiment only $T_{2}$ decay reduces the intensity of the echo. Also in this sequence it is the echo signal which is phase encoded rather than the FID signal since the phase encoding gradient is applied after the $180^{\circ}$ pulse. It is very similar to the original "spinwarp" technique developed by Edelstein et al. (1980).

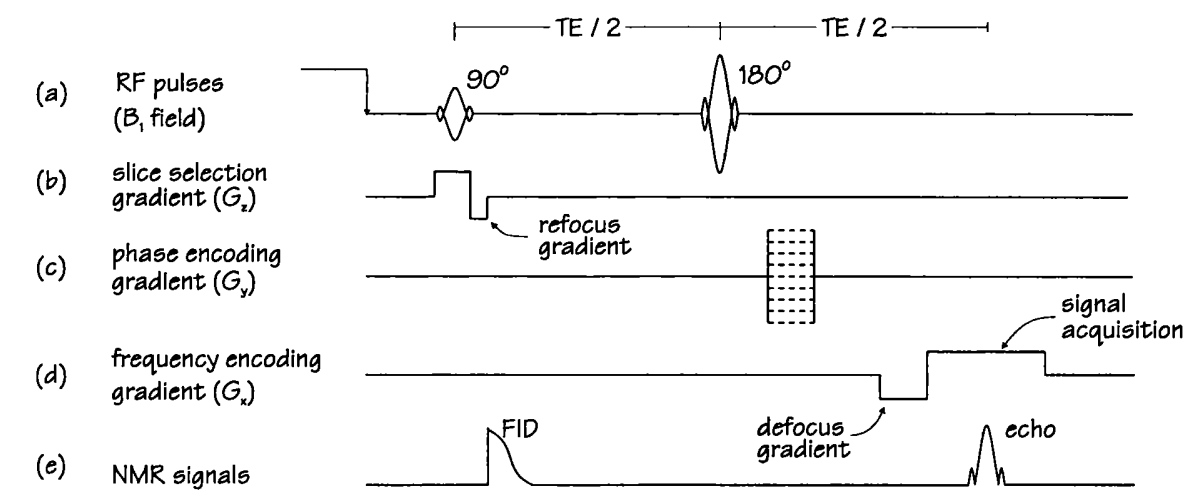

Figure 2.19- The NMR spin-echo pulses sequence

The main advantage of the sequence illustrated in figure 2.19 is that instead of encoding information in the $y$-direction by increasing the time $t_{y}$ the amplitude of $G_{y}$ is increased. The resulting effect is to introduce a phase shift into the echo signal which 
should satisfy the integral condition (Edelstein et al.,1980),

$$
\gamma L_{y} \int_{0}^{\tau} d t G_{y}=2 \pi n
$$

where $L_{y}$ is the total length of the field of view (FOV) in the $y$-direction $\int_{0}^{\tau} \mathrm{dt}$ is the fixed period of time in which $G_{y}$ is applied and $n$ is an integer number. In order to distinguish $N$ regions in the vertical direction, it is necessary to take $N$ signals with $n$ $=-1 / 2 N,-1 / 2 N+1, \ldots,-1,0,1, \ldots, 1 / 2 N-1$.

Thus if for each line of the matrix a different $G_{y}$ is used, the recorded echo signal will have a proportional phase shift. As before, this leads to a modulation of the Fourier components in the frequency domain.

Figure 2.19 (a) shows the RF $90^{\circ}$ and $180^{\circ}$ pulses. The $90^{\circ}$ RF pulse is applied during the slice selection gradient $G_{z}$. Just after the $90^{\circ} \mathrm{RF}$ pulse, a reverse gradient is used to refocus the spins. It compensates for the field gradient $G_{z}$ through the selected slice. Figure 2.20 illustrates the process of phase refocusing. In figure 2.20 because of the gradient field, there are different fields at the slice borders (points 1 and 2 in figure $2.20(a))$ producing a slightly different frequency of precession, figure $2.20(\mathrm{c})$, which reduces the signal induced in the coil. The effect of the reverse gradient is to accelerate the spins at point 1 and decelerate the spins at point 2 bringing them together, or refocusing them.

(a)

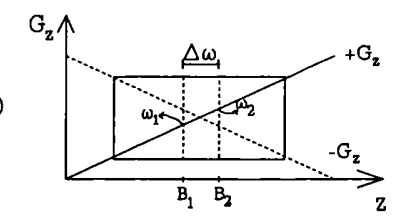

(b)
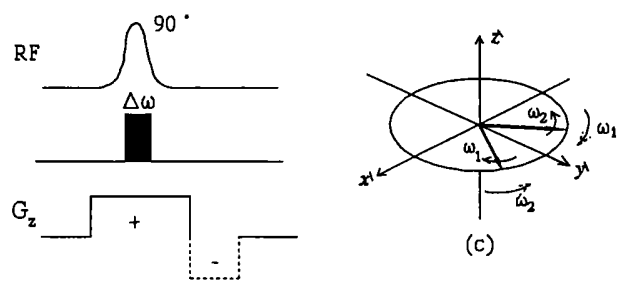

(c)

Figure 2.20-Effects of the refocusing pulse in the net magnetisation.

The $G_{y}$ gradient in figure 2.19(c) is applied during the interval between the RF pulses, each time with a different strength. This encodes the phase in the $y$ direction. After the $180^{\circ} \mathrm{RF}$ pulse, the echo signal is detected in presence of the $G_{x}$ gradient, 
which produces the frequency encoding, in the $x$-direction, figure 2.19(d). Note the presence of a defocus gradient before the signal acquisition $G_{x}$ gradient is applied. This defocus gradient compensates for the spin dephasing caused by the gradient presence.

\subsection{NMR image reconstruction}

This section presents the algorithm used to reconstruct the NMR images. First the principles of NMR signal demodulation and digitisation are briefly described to introduce the actual signal used to reconstruct the NMR images. The treatment required of the time domain samples and the two dimension Fourier transform to reconstruct the images are then reviewed. Since in this work it is the NMR phase images which are used to measure the magnetic field of the electrical current that is propagating within the object, the theory of phase correction is also reviewed. Finally, the algorithms implemented to perform the image reconstruction and phase correction are described. Both signal simulation and an NMR experiment were performed to check the algorithm implementation.

\subsubsection{NMR signal demodulation}

NMR signal demodulation consist of converting the RF spin-echo signal (hundreds of megahertz) into a low frequency (tens of kilohertz) to enable the digitisation process. Figure 2.21, illustrates the quadrature phase detector used to demodulate the NMR signal. Once the weak RF signal, $S(t)$ induced in the coil by the precessing magnetisation is amplified, the next step is to split it into two channels and compare each channel with a reference frequency through a phase sensitivity detector (psd). The demodulation process could be performed by a single psd, but considering the main advantages of the quadrature detection (ability to discriminate between positive and negative frequency and net gain in the signal to noise ratio of a factor of $\sqrt{2}$ (Marshal et al., 1990; Morris, 1986)) two channels are used.

Consider for analysis purposes the signal of a single spin isochromat,

$$
S(t)=k M_{0} \sin \left[\left(\omega_{0}+\delta \omega\right) t+\varphi\right]
$$

where $\omega_{0}$ is the Larmor frequency, $\delta \omega$ is the offset frequency caused by the readout gradient, $k$ is a constant which depends on the sensitivity of the receiver coil and signal amplification, $\varphi$ represents a constant phase shift caused by all the electronics plus the 


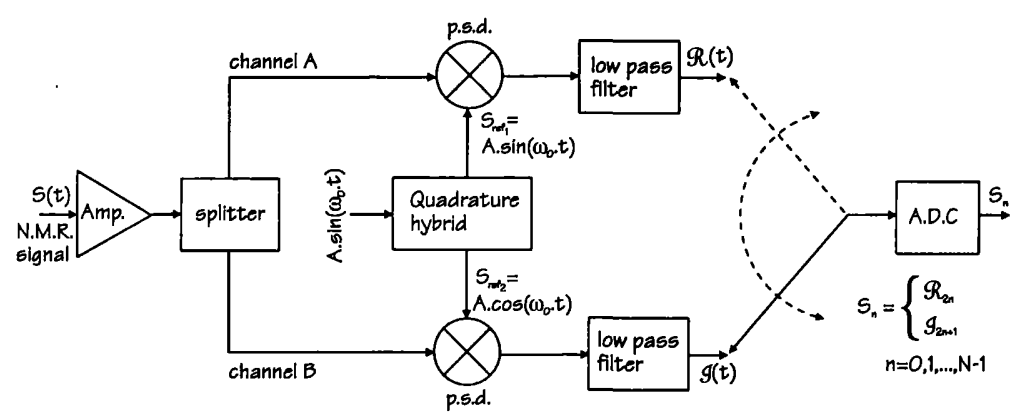

Figure 2.21- The quadrature detector to demodulate the NMR signal and the time sharing ADC converter system.

phase encode gradient steps, and $M_{0}$ is the net magnetisation amplitude, given by equation (2.24).

In figure 2.21 the reference frequency $S_{\text {refl }}$, equal to the Larmor frequency, is given by,

$$
S_{r e f_{1}}=A \sin \left(\omega_{0} t\right)
$$

and $S_{\text {ref2 }}$, obtained through a phase-shift of $90^{\circ}$ on the Larmor frequency signal, is given by,

$$
S_{r e f_{2}}=A \cos \left(\omega_{0} t\right)
$$

Essentially, each psd in figure 2.21 demodulates the NMR signal by multiplying it by its respective reference frequency. For the sine reference channel, $\mathfrak{R}(t)$ in figure 2.21 is given $b^{7}$,

$$
\begin{aligned}
\mathfrak{R}(t) & =\left\{A \sin \left(\omega_{0} t\right)\right\}\left\{k M_{0} \sin \left[\left(\omega_{0}+\delta \omega\right) t+\varphi\right]\right\} \\
& =\frac{A k M_{0}}{2}\left\{\cos (\delta \omega t+\varphi)-\cos \left(2 \omega_{0} t+\delta \omega t+\varphi\right)\right\}
\end{aligned}
$$

and for the cosine reference channel, $\mathfrak{I}(t)$ in figure 2.21 is given $b^{8}$,

$$
\begin{aligned}
\mathfrak{I}(t) & =\left\{A \cos \left(\omega_{0} t\right)\right\}\left\{k M_{0} \sin \left[\left(\omega_{0}+\delta \omega\right) t+\varphi\right]\right\}= \\
& =\frac{A k M_{0}}{2}\left\{\sin (\delta \omega t+\varphi)+\sin \left(2 \omega_{0} t+\delta \omega t+\varphi\right)\right\}
\end{aligned}
$$

\footnotetext{
${ }^{7}$ Since $\quad \sin A \sin B-\frac{1}{2}\{\cos (A-B)-\cos (A-B)\}$

${ }^{8}$ Since $\quad \sin A \cos B=\frac{1}{2}(\sin (A-B)-\sin (A+B)\}$
} 
The high frequencies components in equations (2.57) and (2.58) are readily removed by a low pass filter and the results are the real, $\mathfrak{R}(t)$, and imaginary, $\mathfrak{I}(t)$, time domain components,

$$
S(t)=k M_{0}\{\cos (\delta \omega t+\varphi)+i \sin (\delta \omega t+\varphi)\}
$$

If the two channels do not differ by exactly $90^{\circ}$ in phase, then the real and imaginary data sets will no longer be perfectly independent and a complex Fourier transform of those data sets will produce a spectrum with image peaks (see item 2.3.3).

\subsubsection{Signal digitisation}

As described by equation (2.59) quadrature detection denotes the simultaneous detection of two signals differing in phase by $90^{\circ}$. For practical limitations (two quadrature channels identical with respect to dc offset, gain, phase and time delay between the raw signal and analog-to-digital (ADC) output (Marshal et al., 1990)) a quadrature detection scheme is often accomplished by time-sharing a single ADC, as illustrate in figure 2.21 .

This means that there is a delay time between the real and imaginary samples. Generally the imaginary component is sampled in the middle of the two real ones. The real and imaginary components after conversion are stored in one array where the even samples are the real components $(\mathfrak{R})$ and the odd samples are the imaginary components (I). For $N$ real samples and $N$ imaginary samples, the $2 N$ array is $\mathfrak{R}_{0} \mathfrak{I}_{1} \mathfrak{R}_{2} \mathfrak{I}_{3} \mathfrak{R}_{4} \mathfrak{I}_{5} \mathfrak{R}_{6}$ $\mathfrak{I}_{7} \ldots \mathfrak{R}_{2 N-2} \mathfrak{I}_{2 N-1}$, or splitting the even and odd samples the spin-echo $S_{n}$ may be expressed as:

$$
S_{n}=\left\{\begin{array}{l}
\mathfrak{R}_{2 n} \\
\mathfrak{I}_{2 n+1}
\end{array} \quad \text { for } n=0,1,2, \ldots, N-1\right.
$$

The next section describes the signal treatment required before the actual FT of the signal $S_{n}$.

\subsubsection{The two-dimensional discrete Fourier transform}

The Discrete Fourier Transform (DFT) of $N$ sample points of a time domain function $S_{n}$, sampled at an interval $T$, produces $N$ discrete components of frequency $F_{k}$ given by,

$$
F_{k}=\frac{1}{N} \sum_{n=0}^{N-1} S_{n} e^{-i \frac{2 \pi n k}{N}} \text { for } \quad k=-\frac{N}{2}, \ldots, \frac{N}{2}
$$


where $S_{n}=S(n T)$ and the actual frequency value is equal to $f=k /(N T)$.

The result of a DFT is a complex quantity equal to,

$$
F_{k}=R_{k}+i I_{k}=\left|F_{k}\right| e^{i \theta_{k}}
$$

where, $R_{k}$ is the real part and $I_{k}$ the imaginary part of the Fourier transform, $F_{k}$ is the amplitude or Fourier spectrum of $S_{n}$, given by,

$$
\left|F_{k}\right|=\sqrt{R_{k}^{2}+I_{k}^{2}}
$$

and $\theta_{k}$ is the phase angle of the Fourier transform, given by,

$$
\theta_{k}=\tan ^{-1}\left[\frac{I_{k}}{R_{k}}\right]
$$

Consider, again for the purpose of analysis, that the $2 N$ samples in equation (2.60) as cosine and sine respectively, are given by,

$$
S_{n}=\left\{\begin{array}{l}
\Re_{2 n}=A \cos \left[\frac{2 \pi f n}{N}\right] \\
\mathfrak{I}_{2 n+1}=A \sin \left[\frac{2 \pi f(n+1 / 2)}{N}\right]
\end{array} \text { for } n=0,1,2, \ldots, N-1\right.
$$

For a normal complex Fourier transform applied to this signal the resulting spectrum, formally developed in Appendix A, is given by equation (2.66).

$$
F_{k}=\frac{1}{2}\left\{\left[1+\cos \frac{\pi f}{N}\right] \delta_{(k-f)}+\left[1-\cos \frac{\pi f}{N}\right] \delta_{(k+f)}+i\left(\sin \frac{\pi f}{N}\right)\left[\delta_{(k-f)}+\delta_{(k+f)}\right]\right\}
$$

To show the spectrum of $S_{n}$, the real and imaginary images were simulated according to equation (2.65) with $f=20 \mathrm{~Hz}, N=256$ and $A=5$. Also an offset of 1 was added to the cosine signal to produce a zero frequency component in the spectrum. Figures 2.22(a) and (b) show the simulated real and imaginary images. A program using the Fourier transform routines from Numerical Recipes (Press et al., 1992) was implemented to compute the spectrum (section 2.3.5).

Since there is no change among the lines in figure 2.22 , the Fourier transform produces just a vertical line in the pixel position that corresponds to the simulated frequency. Therefore, in figure 2.23 just a profile of the real and imaginary images are 


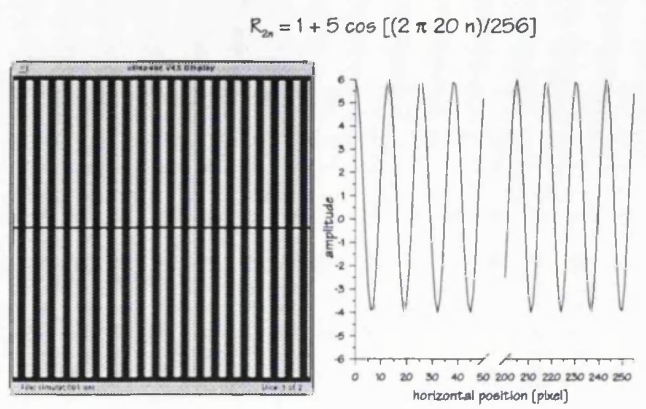

(a)

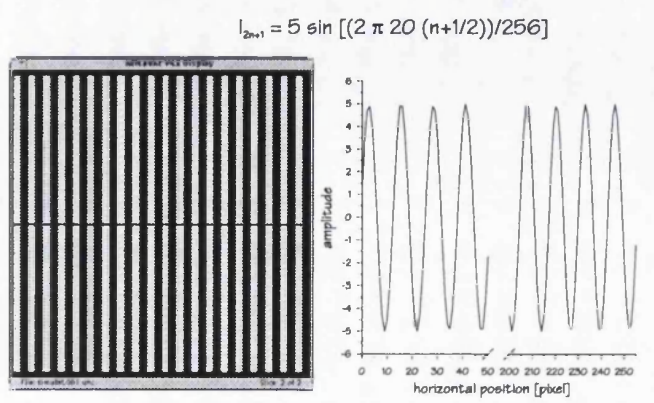

(b)

Figure 2.22 - (a) Real and (b) Imaginary time domain components. Both were simulated according to equation (2.65) to simulate the spectrum of $S_{n}$.

shown. It matches the theoretical spectrum from equation (2.66) with four $\delta$-functions, two in the real side with different amplitudes and two in the in the imaginary side with the same amplitude.

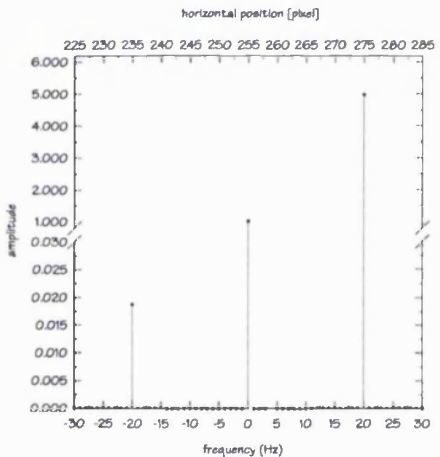

(a)

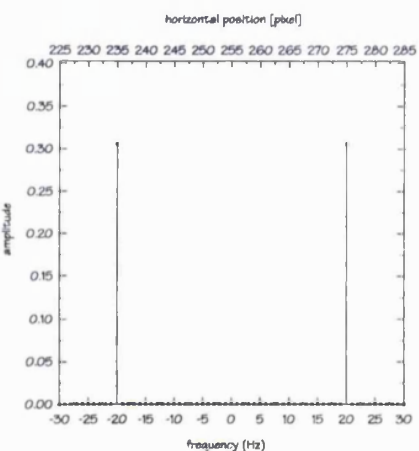

(b)

Figure 2.23-(a) Real and (b) Imaginary spectrum of the DFT of the time signal shown in figure 2.22.

The correct spectrum expected for this case where the real component is a cosine and the imaginary a sine, both sampled simultaneously be derived as in equation (2.67),

$$
\begin{aligned}
& F_{k}=\frac{1}{N} \sum_{n=0}^{N-1} S(t) e^{-i \frac{2 \pi k n}{N}} \\
& F_{k}=\frac{1}{N} \sum_{n=0}^{N-1}\left[\cos \left(\frac{2 \pi f n}{N}\right)+i \sin \left(\frac{2 \pi f n}{N}\right)\right] e^{-i \frac{2 \pi k n}{N}} \\
& F_{k}=\frac{1}{N} \sum_{n=0}^{N-1}\left[\frac{e^{i \frac{2 \pi f n}{N}}+e^{-i \frac{2 \pi f n}{N}}}{2}+i\left(\frac{e^{i \frac{2 \pi f n}{N}}-e^{-i \frac{2 \pi f n}{N}}}{2 i}\right)\right] e^{-i \frac{2 \pi k n}{N}} \\
& F_{k}=\frac{1}{N} \sum_{n=0}^{N-1} \frac{1}{2}\left[2 e^{i \frac{2 \pi f n}{N}}\right] e^{-i \frac{2 \pi k n}{N}}=\frac{1}{N} \sum_{n=0}^{N-1} e^{-i \frac{2 \pi n(k-f)}{N}}=\delta_{k-f}
\end{aligned}
$$


which is just one peak in the real part of the Fourier spectrum at the frequency $f$.

An NMR experiment was performed with one of the spectrometer calibration phantoms to understand the image artifacts produced by a sequential data acquisition. The experiment collected 256 spin-echo signals each one with a different phase encode gradient. Each spin-echo signal was sampled 256 times, with a sample frequency equal to $25 \mathrm{kHz}$. Producing an image matrix size of 256 by 256 pixels. First a normal complex DFT was applied to all rows, followed by the columns. Figures 2.24(a) and (b) are the real and imaginary parts of the time domain data (signals $\Re_{2 n}$ and $\mathfrak{I}_{2 n+1}$ in figure 2.21). Figures 2.25(a) and (b) show images of the real and imaginary parts of the spectrum and figure 2.26(a) and (b) are the corresponding phase and magnitude images. These images show an artifact that looks like a shadow and will be referred to as a ghost in the phantom image.

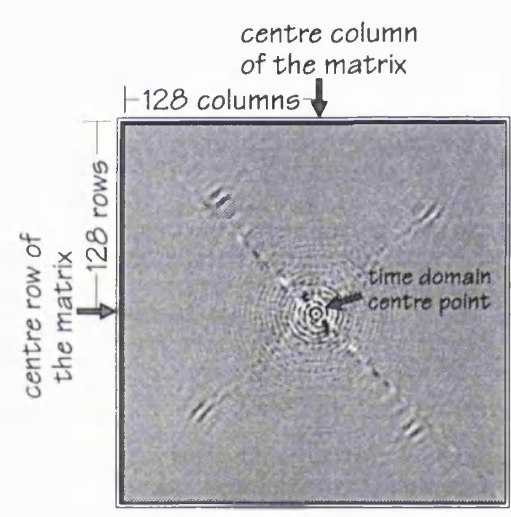

(a)

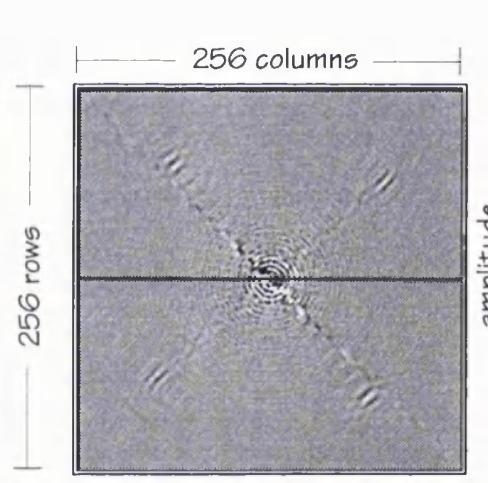

(b)

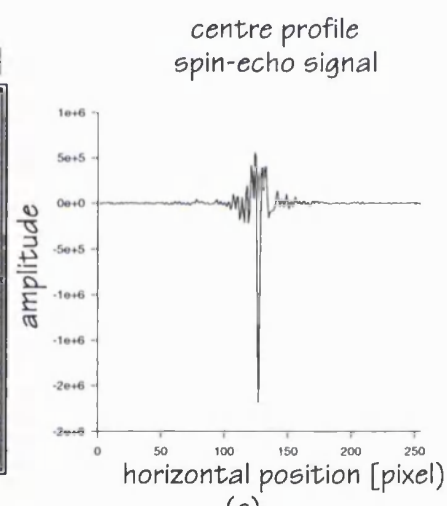

(c)

Figure 2.24 - Real (a) and Imaginary (b) time domain spin-echo signals of the phantom experiment. Echo time was $120 \mathrm{~ms}$, Repetition time $700 \mathrm{~ms}$ and slice thickness $5 \mathrm{~mm}$. (c) image profile of figure (b).
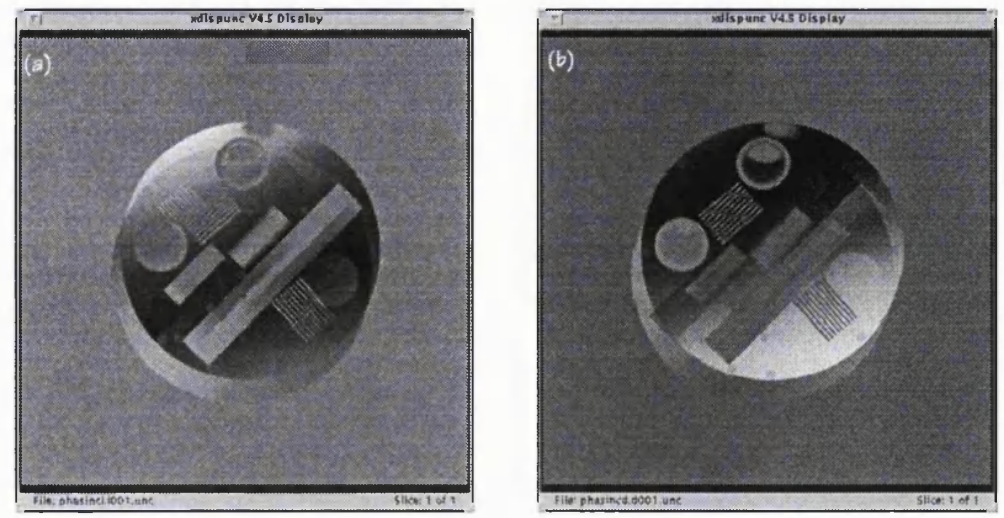

Figure 2.25 - Images from the (a) Real and (b) Imaginary parts of the spectrum of the signal shown in figure 2.24 . 

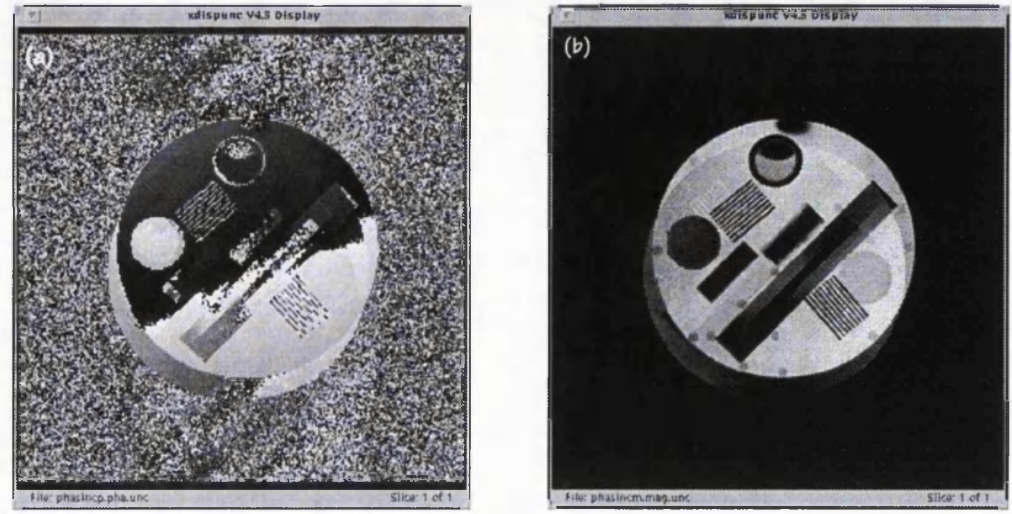

Figure 2.26 - (a) Phase and (b) Magnitude images of the signal shown in figure 2.24.

\subsubsection{The Redfield and Kunz treatment}

To eliminate these ghosts artifacts shown in figure 2.26 and correct the spectrum, Redfield and Kunz (1975) suggested a signal treatment before the actual Fourier transform. The treatment consists of multiplying the $2 N$ time domain samples by $\{+1-$ $1-1+1+1-1-1+1+1 \ldots\}$, which can be written as in equation (2.68),

$$
S_{n}{ }^{\prime}=\left\{\begin{array}{l}
\mathfrak{R}_{2 n}{ }^{\prime}=+(-1)^{n} \mathfrak{R}_{2 n} \\
\mathfrak{I}_{2 n+1},=-(-1)^{n} \mathfrak{I}_{2 n+1}
\end{array} \quad \text { for } n=0,1, \ldots, n-1\right.
$$

and applying a real Fourier transform ${ }^{9}$ to the $2 N$ samples $S_{n}{ }_{n}$. Therefore,

$$
F_{k}=\frac{1}{N} \sum_{n=0}^{2 N-1} S_{n}, e^{-i \frac{2 \pi k n}{2 N}} \quad \text { for } \quad k=-\frac{N}{2}, \ldots,+\frac{N}{2}
$$

which may be split into even and odd samples,

and decomposed into,

$$
F_{k}=\frac{1}{N}\left\{\sum_{n=0}^{N-1} \Re_{2 n}, e^{-i \frac{2 \pi k 2 n}{2 N}}+\sum_{n=0}^{N-1} \mathfrak{I}_{2 n+1}, e^{-i \frac{2 \pi k(2 n+1)}{2 N}}\right\}
$$

$$
F_{k}=\frac{1}{N}\left\{\sum_{n=0}^{N-1} \Re_{2 n}, e^{-i \frac{2 \pi n k}{N}}+\sum_{n=0}^{N-1} \mathfrak{I}_{2 n+1},\left\{e^{-i \frac{2 \pi k 2 n}{2 N}} e^{\left.-i \frac{-i 2 \pi k}{2 N}\right\}}\right\}\right.
$$
thus,

In a Real Fourier transform the imaginary part of the time domain signal is set equal to zero. 


$$
F_{k}=\frac{1}{N}\left\{\sum_{n=0}^{N-1}\left[\mathfrak{R}_{2 n}^{\prime}+\mathfrak{I}_{2 n+1}, e^{-i \frac{\pi k}{N}}\right] e^{-i \frac{2 \pi n k}{N}}\right\}
$$

substituting the real and imaginary parts by the cosine/sine signals respectively and applying the Redfield and Kunz treatment,

$$
F_{k}=\frac{1}{N}\left\{\sum_{n=0}^{N-1}(-1)^{n}\left[\cos \left(\frac{2 \pi f n}{N}\right)-\sin \left(\frac{2 \pi f(n+1 / 2)}{N}\right) e^{-i \frac{\pi k}{N}}\right] e^{-i \frac{2 \pi n k}{N}}\right\}
$$

which can be developed as in Appendix B, resulting,

$$
F_{k}=\frac{1}{N} \sum_{n=0}^{N-1} \frac{1}{2}\left\{\left(1+e^{-i \frac{\pi(k-f-N / 2)}{N}}\right) e^{-i \frac{2 \pi n(k-f-N / 2)}{N}}+\left(1-e^{-i \frac{\pi(k+f-N / 2)}{N}}\right) e^{-i \frac{2 \pi n(k+f-N / 2)}{N}}\right\}
$$

In equation (2.74) for $k=(N / 2-f)$ the second round bracket is equal to zero, exactly where the dirac $\delta$ functions exists, and for $k=(N / 2+f)$ the first round bracket is equal to two, resulting in:

$$
F_{k}=\frac{1}{2 N} 2 N \delta_{(k, N / 2+f)}=\delta_{(k, N / 2+f)}
$$

Figure 2.27 is the resulting spectrum for the same input cosine/sine signal shown in figure 2.22, but after the Redfield and Kunz treatment. This shows the same amplitudes for the simulated sine/cosine signals and for offset level as applied in equation (2.65).
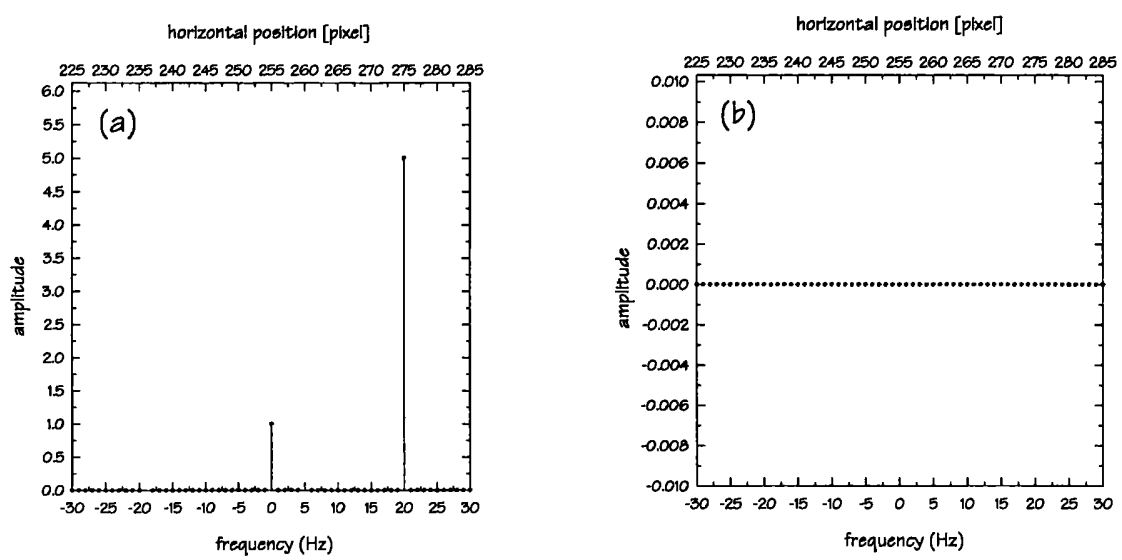

Figure 2.27- Real and imaginary spectrum, (a) and (b) respectively, of the DFT of the time domain signal shown in figure 2.22 after the Redfield and Kunz treatment

Using the same time domain data of the phantom shown in figures 2.24 , the 
Redfield and Kunz treatment was applied for each spin-echo signal. After each spin echo was Fourier transformed, a normal complex FT was applied to each column. Figures 2.28(a) and (b) illustrate the real and imaginary images and figures 2.29(a) and b are the phase and magnitude images. To show the spectrum of frequencies present in the NMR images a horizontal centre profile of figure 2.28 is plotted in figure 2.30 .
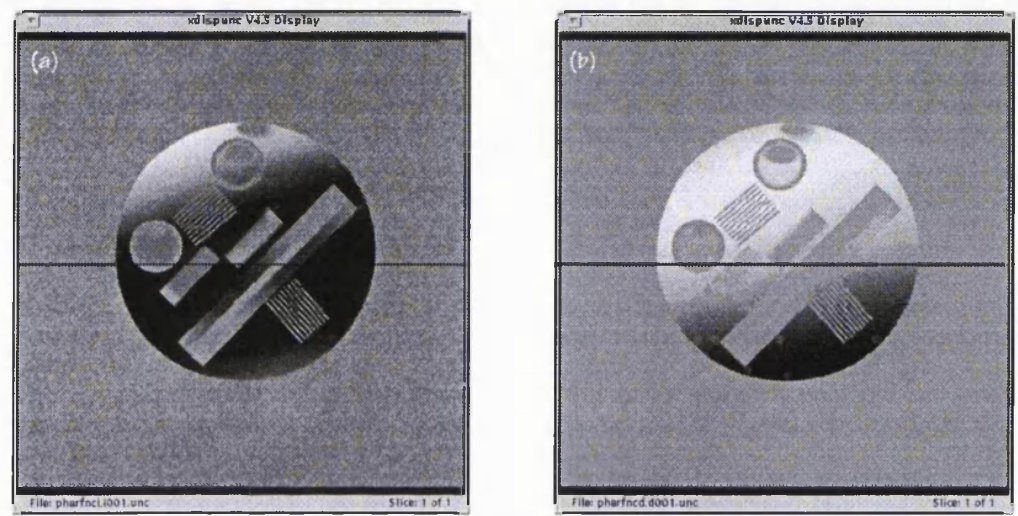

Figure 2.28 - Real (a) and Imaginary (b) images of the time domain signal shown in figure 2.24. The centre lines indicate the profile position shown in figure 2.30 .
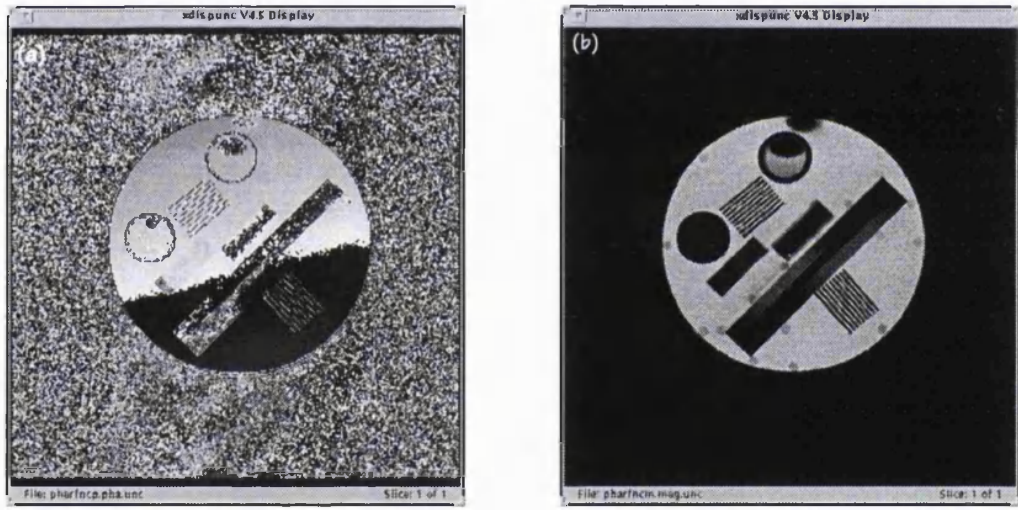

Figure 2.29 - Phase (a) and magnitude (b) images of the time signal shown in figure 2.24

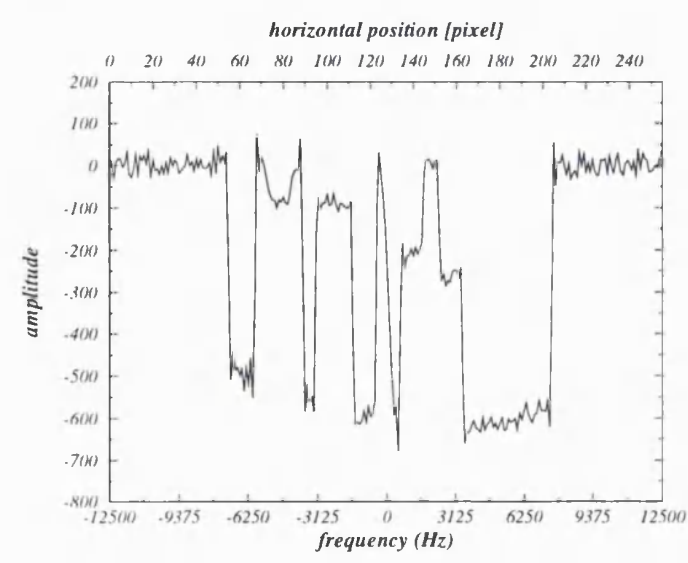

(a)

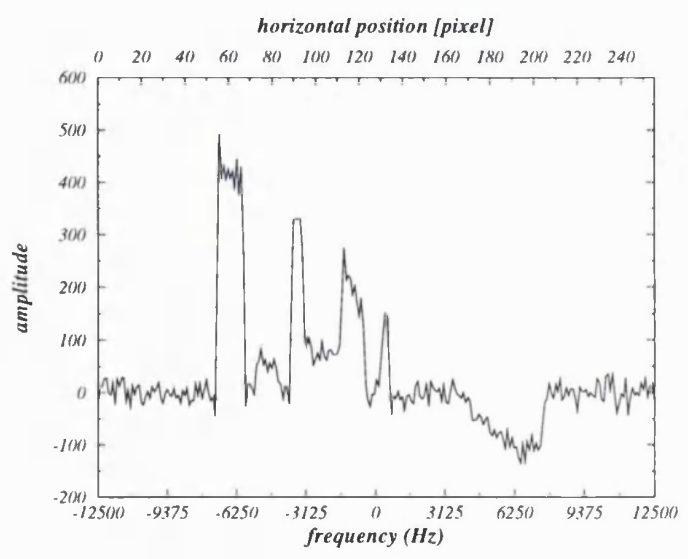

(b)

Figurer 2.30 - Horizontal centre profile of the real and imaginary images shown in figure 2.28. 
The algorithm suggest by Redfiled and Kunz (1975), is strictly only applicable for the case where the imaginary time domain part is sampled right in the middle of two real components. It is worth mentioning here that Keller and Haeberlen (1988) suggested an algorithm where the time delay for the imaginary sample can be anywhere within the dwell time.

\subsubsection{The theory of NMR phase-correction}

The aim of phase correction in NMR phase images is to reduce the unnecessary $2 \pi$ phase jumps as illustrated in figure 2.29 (a) where the phase goes from $-\pi$ (black region in the image) to $+\pi$ (white region in the image). Besides, for a spin-echo sequence the absorption mode in figure 2.28 (a) should be theoretically maximized and the dispersion mode in figure 2.28 (b) zero. It must also be born in mind that phase correction does not produce any effect on the magnitude image (figure 2.29(b)), but it does help to produce phase data which is much easier to work with. Finally, since working with phase information requires a phase reference, it is crucial to repeat the same phase correction for all the phase images that will be compared with the starting phase reference. To understand the phase correction principle a mathematical approach is briefly introduced.

Equation (2.54) gives the NMR signal after demodulation. Adding to this equation an exponential term, with a constant time $\tau$, related to the relaxation caused by $T_{2}{ }^{*}$ and considering initially just the $j^{\text {th }}$ voxel, the NMR signal $S_{j}(t)$ is,

$$
S_{j}(t)=A_{j} e^{-i\left(\delta \omega t+\varphi_{j}\right)} e^{-\frac{t}{\tau}}
$$

where, $A_{j}=\left(A_{r} k M_{0}\right) / 2, A_{r}$ is the amplitude of the reference signals for the quadrature demodulation, $k$ is the amplifier constant, $\varphi_{j}$ is the phase shift produced by the receiver channels at $t=t_{0}$ and $M_{0}$ is the net magnetisation vector at $t=t_{0}$.

An additional phase shift is introduced in equation (2.76) by a time delay $t_{d}$ between the excitation (RF pulse) and the actual measurement (spin-echo signal acquisition) of the spins response,

$$
S_{j}\left(t+t_{d}\right)=A_{j} e^{-i\left[\delta \omega\left(t+t_{d}\right)+\varphi_{j}\right]} e^{-\frac{\left(t+t_{d}\right)}{\tau}}
$$

The $S(t)$ signal is Fourier-transformed to calculate the spectrum $F_{j}(\omega)$, 


$$
F_{j}(\omega)=\int_{-\infty}^{+\infty} S_{j}(t) e^{-i \omega t} d t=\int_{t_{d}}^{-\infty} S_{j}(t) e^{-i \omega t} d t=\int_{0}^{+\infty} S_{j}\left(t+t_{d}\right) e^{-i \omega\left(t+t_{d}\right)} d t
$$

substituting the NMR signal, equation (2.77),

$$
F_{j}(\omega)=\int_{0}^{+\infty} A_{j} e^{+i\left[\delta \omega_{j}(t+t d)+\varphi_{j}\right]} e^{-\frac{t+t_{d}}{\tau}} e^{-i \omega t} d t
$$

which may be developed to,

$$
F_{j}(\omega)=A_{j} e^{i \delta \omega_{j} t_{d}} e^{i \varphi_{j}} e^{-\frac{t_{d}}{\tau}} \int_{0}^{+\infty} e^{-\frac{\left[-i\left(\delta \omega_{j}-\omega\right) \tau+1\right] t}{\tau}} d t
$$

and solving the integral,

$$
F_{j}(\omega)=A_{j} e^{i \delta \omega_{j} t_{d}} e^{i \varphi_{j}} e^{-\frac{t_{d}}{\tau}} \frac{\tau}{1-i\left(\delta \omega_{j}-\omega\right) \tau}
$$

To isolate the real part from the imaginary part, we multiply the top and bottom by the conjugate of the denominator resulting in,

$$
\begin{aligned}
& F_{j}(\omega)=e^{i \varphi_{j}^{\prime}} A_{j}^{\prime}\left[\frac{\tau}{1+\tau^{2}\left(\delta \omega_{j}-\omega\right)^{2}}+i\left(\frac{\tau^{2}\left(\delta \omega_{j}-\omega\right)}{1+\tau^{2}\left(\delta \omega_{j}-\omega\right)^{2}}\right)\right] \\
& \text { where } \varphi_{j}^{\prime}=\varphi_{j}+\delta \omega_{j} t_{d} \text { and } A_{j}^{\prime}=A_{j} e^{-\frac{t_{d}}{\tau}}
\end{aligned}
$$

The bracketed expressions in equation (2.82) are the normalised Lorentzian dispersion and absorption $[D(\omega)$ and $A(\omega)$ in equation (2.83)] which occur universally in spectroscopy (Marshall, 1990).

$$
A(\omega)=\frac{\tau}{1+\left(\omega_{0}-\omega\right)^{2} \tau^{2}} \quad D(\omega)=\frac{\left(\omega_{0}-\omega\right) \tau^{2}}{1+\left(\omega_{0}-\omega\right)^{2} \tau^{2}}
$$

Expanding the exponential term outside the square brackets in equation (2.82),

$$
\begin{aligned}
& F_{j}(\omega)=A_{j}^{\prime}\left\{\left[A_{j}(\omega)+i D_{j}(\omega)\right] e^{i \varphi}\right\} \\
& F_{j}(\omega)=A_{j}^{\prime}\left\{A_{j}(\omega) \cos \varphi-D_{j}(\omega) \sin \varphi+i\left[A_{j}(\omega) \sin \varphi+D_{j}(\omega) \cos \varphi\right]\right\}
\end{aligned}
$$

where the real, $R_{j}$, and imaginary, $I_{j}$ components of the spectrum are,

$$
\begin{aligned}
R_{j} & =\left\{A_{j}(\omega) \cos \left(\varphi_{j}\right)-D_{j}(\omega) \sin \left(\varphi_{j}\right)\right\} A_{j}^{\prime} \\
I_{j} & =\left\{A_{j}(\omega) \sin \left(\varphi_{j}\right)-D_{j}(\omega) \cos \left(\varphi_{j}\right)\right\} A_{j}^{\prime}
\end{aligned}
$$

As can be seen in equation (2.85). The real and imaginary components of the 
spectrum after the Fourier transform are a mixture of both the absorption and the dispersion modes. To obtain the pure absorption and dispersion components, the measured spectrum needs to be phase-corrected by $\varphi_{j}$, according to equations (2.86).

$$
\begin{aligned}
& A_{j}(\omega)=R_{j}(\omega) \cos \left(\varphi_{j}\right)+I_{j} \sin \left(\varphi_{j}\right) \\
& D_{j}(\omega)=I_{j}(\omega) \cos \left(\varphi_{j}\right)-R_{j} \sin \left(\varphi_{j}\right)
\end{aligned}
$$

In the NMR experiment, following the excitation, all spin magnetisations are excited simultaneously, and thus the spin-echo signal induced in the image coil from the spins at each voxel begin with the same initial phase (say, zero, corresponding to a wave that starts as a cosine at $t=0$ ). If there is a time-delay, $t_{d}$, between excitation and detection, then there will be a corresponding phase shift $\omega t_{d}$ which is linearly proportional to each frequency component.

Therefore, the phase distortion should be eliminated by phase correcting the contribution of each individual line over the complete spectral bandwidth by its phase distortion $\varphi_{j}$ according to equation (2.86).

The effect of the delay time between the excitation and detection can only be observed in the phase images (figure 2.29(a)). By applying equation (2.86) it is possible to obtain a very flat phase image where most of the signal is concentrated in the absorption mode. The algorithm implemented to perform the phase correction (described in the next section) was applied to the phantom image showed in figure 2.24. Figures 2.31 (a) and (b) show the real and imaginary images after the phase correction and figure 2.32(a) and (b) shown the phase and magnitude spectrum images.
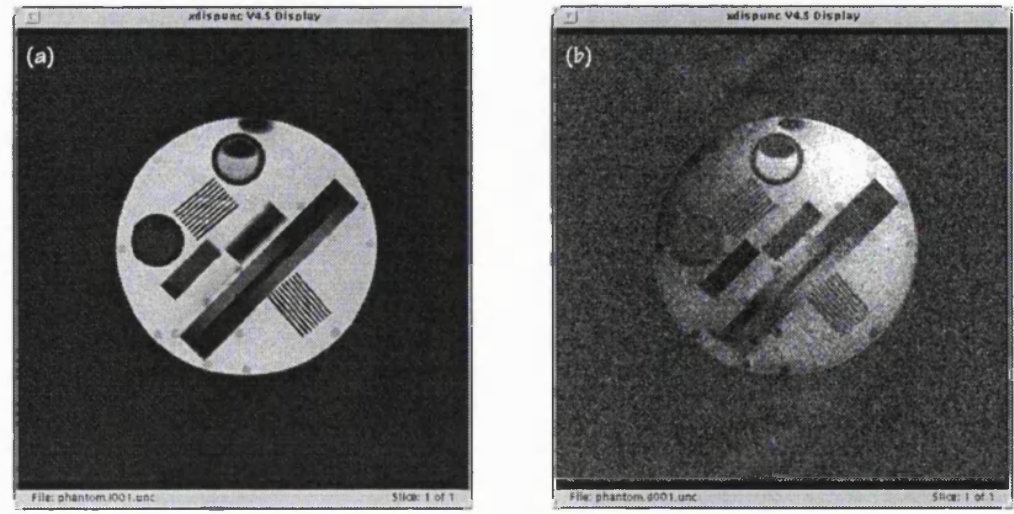

Figure 2.31 - a) Real and b) Imaginary images after the phase correction. 

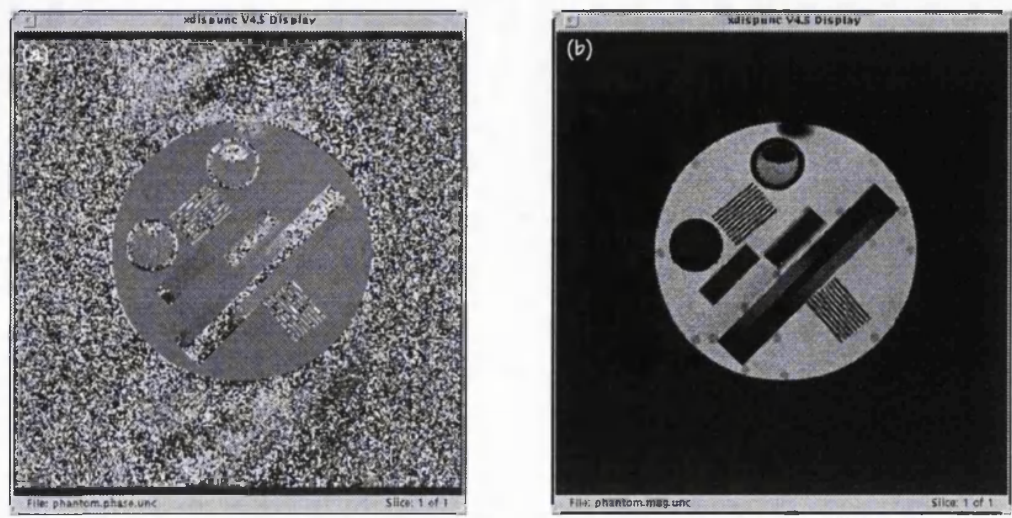

Figure 2.32 - a) Phase and b) magnitude images from the real and imaginary images shown in figure 2.31 .

\subsubsection{The Fourier transform and the phase-correction algorithms}

Although the Bruker software has of course routines to perform the image reconstruction and phase correction, we decided to write our own programs to execute these tasks on the workstations. The reasons for this were partly because of the poor explanation in the manuals about how the Bruker routines executed the phase correction, but also because of an apparent intermittent error in the phase correction. Sometimes in one experiment the machine would produce nice flat phase images and sometimes a completely undulating phase stripe. In addition, the Bruker System is very slow and the procedure used to estimate the phase parameters takes quite a long time.

The first critical step for image reconstruction when the phase information is required is to find exactly the time domain centre point, as indicated by the arrow in figure 2.24(a). This point corresponds to the time zero and is the first point to be passed to the DFT algorithm. If the centre point is too far away from the first point, the phase image will consist of black and white stripes and it will be very difficult to correct this in the frequency domain. The time domain centre point corresponds to the point where the signal is maximum (the isocentre of the phase encode and readout gradients). Once this is found the next step is to match it with the centre of the image matrix (for example for a 256 by 256 matrix the centre of the time data should be at the coordinates $128,128)$, and perform the DFT. A software routine was written to implement this procedure to simplify the FT algorithms.

To perform the DFT the Numerical Recipes (Press et al. 1992) routines "drealft.c" and "dfourl.c" were used. They perform a double precision Fast Fourier 
Transform (FFT). The algorithm for the image reconstruction is divided into 11 steps: 1- $\quad$ First, the time domain Bruker file is converted to the workstation format. Briefly, the Bruker hardware uses a processor word of 3 bytes ( 24 bits) where the first byte on the right is the most significant and the last on the left the least significant. These data are read byte by byte and conveniently converted to a word of 4 bytes in the workstations as illustrated in figure 2.33;

(a)

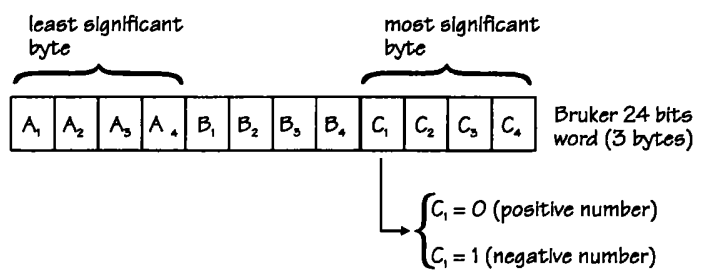

$$
\begin{array}{|l|l|l|l|l|l|l|l|l|l|l|l|l|l|l|l|}
\hline 0 & 0 & 0 & 0 & 0 & C_{2} & C_{3} & C_{4} & B_{1} & B_{2} & B_{3} & B_{4} & A_{1} & A_{2} & A_{3} & A_{4} \\
3 & 32 \text { bits positive } \\
\text { number (4 bytes) }
\end{array}
$$

(b)

\begin{tabular}{|l|l|l|l|l|l|l|l|l|l|l|l|l|l|l|l|}
\hline 1 & 1 & 1 & 1 & 1 & $C_{2}$ & $C_{3}$ & $C_{4}$ & $B_{1}$ & $B_{2}$ & $B_{3}$ & $B_{4}$ & $A_{1}$ & $A_{2}$ & $A_{3}$ & $A_{4}$ \\
$\begin{array}{l}32 \\
\text { number (4 bytes) }\end{array}$ \\
\hline
\end{tabular}

Figure 2.33- a) The 3 bytes word structure used by the NMR Bruker system. $b)$ The 4 bytes word of the workstations structure for a positive number conversion and for a negative number conversion.

2- After the conversion, two time domain matrixes are stored, (real and imaginary). The time domain centre point for the real image is found, and by moving the data set, matched with the matrix centre. Both, real and imaginary images must be moved equally;

3- Split each row of the real and imaginary matrix in the middle, and swap the right and left blocks (this procedure is required by the Numerical Recipes routines);

4- for each matrix row, combine the real and imaginary samples into one array producing a matrix as in equation (2.87);

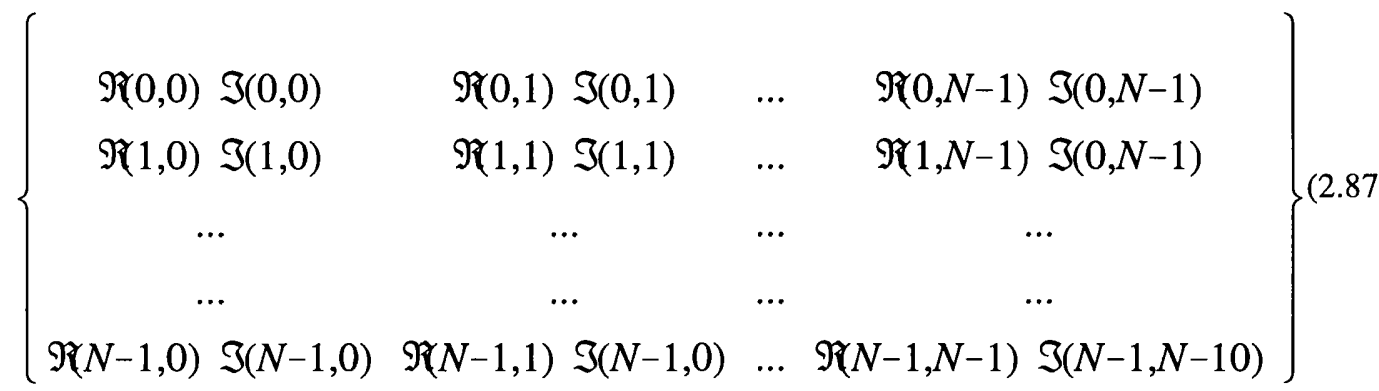

5- for each row in equation (2.87) apply the Redfield and Kunz treatment followed by the real DFT as described in section 2.3.3.1; 
6- repeat step 3;

7- the result of the real DFT is a complex number, apply the phase correction, equation (2.86), as described later in this section;

8- $\quad$ split each column in the middle and swap the top and bottom blocks;

9- apply a normal complex DFT to each column;

10- repeat step 8;

11- apply again the phase correction algorithm;

The phase correction is divided into a zero and a first order phase correction, and both are applied after the row DFT and after the column DFT. The zero and the first order phase correction values are determined for the centre row (step 7) or column (step 11) of the real and imaginary spectrum images, and then repeated to the rest of the image.

The zero order phase correction consist of finding the right angle to apply in equation (2.86) to all pixels along the image. The technique implemented is that suggested by Ernst (1969) and uses the integral intensity criterion originally applied to NMR spectroscopy. When correctly phased, the dispersion mode signal has a vanishingly small integral, whereas the absorption mode signal has a non-zero value. The ratio between these intensities integrals gives the zero order phase correction,

$$
\theta_{0}=\tan ^{-1}\left(\frac{\int I(\omega) d \omega}{\int R(\omega) d \omega}\right)
$$

The baseline offset can be a problem when computing the integral values. To avoid this, the average value over the first and last few points of the profile are subtracted before the integration. Also to differentiate the noise from the signal during integration a threshold value is set equal to three times the noise standard deviation of the same points averaged to the baseline correction.

Once the zero order phase correction has been applied to all pixels according to equation (2.86), the next step is to fit a linear equation to the phase centre row or column profile. This equation is used to determine the phase correction as a function of pixel position and is applied to all rows or columns along the image.

The zero and first order parameters for the phase correction after the row or 
column DFT are determined for an image acquired with no electrical current. These values are then stored in a file and used to correct the phase images acquired with the electric current on. These procedures produce phase images where the phase changes are those produced by the electric current magnetic field.

\subsection{The Bruker spin-echo sequence}

In the previous section the spin-echo pulse sequence was described. It is slightly different from the actual spin-echo sequence used in the electrical current measurement experiment, which is presented here. The Bruker spin-echo sequence is called multi-slice multi-echo or MSVE. It allows us to acquire a sequence of slices with different echos as its name implies, although for the current experiment just one slice is acquired at a time, because it is necessary to synchronise the electrical current pulses with the NMR pulse sequence (see section 3.1). The time diagram of the Bruker MSVE pulse sequence is given in figure 2.34 .

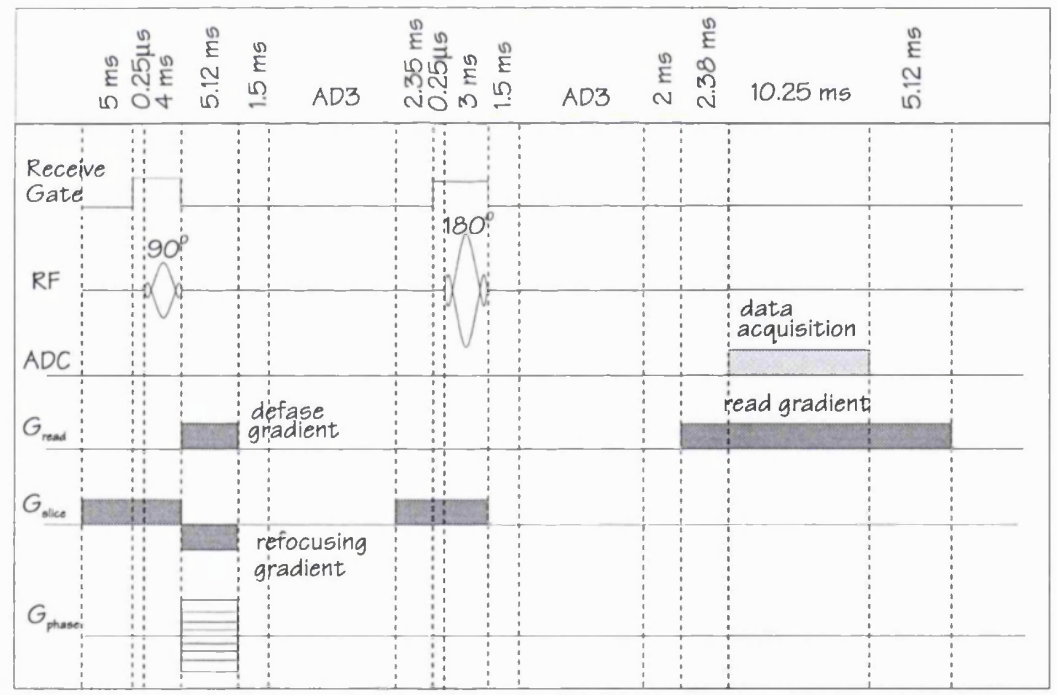

Figure 2.34- The Multi-slice Multi-echo pulse sequence used in the experiments.

The major difference between the MSVE and the spin-echo sequence shown in figure 2.19 is the position of the phase encoding gradient. The Bruker system applies the phase encode gradient just after the $90^{\circ} \mathrm{RF}$ pulse. Because of the phase encoding position, a straight line used to appear in the centre of the NMR image. At the beginning of this project this line was eliminated by averaging two images, but this procedure required a longer period of scanning.

The reason for this line can be understood by considering the RF pulse 
imperfections. This is not a perfectly pulse and therefore it will not rotate the selected spins by exactly $90^{\circ}$ as would be expected. Instead some spins are left behind and may have their rotation equalised to $90^{\circ}$ by the equally imperfect $180^{\circ}$ pulse. It may also happen that some spins may be rotated by exactly $90^{\circ}$ by the edges of the $180^{\circ} \mathrm{RF}$ pulse. As shown in figure 2.34, after of the $90^{\circ} \mathrm{RF}$ pulse all the spins within the selected slice, that will contribute to the NMR signal are spatially encoded by the phase encoding gradient. But after the refocusing RF pulse some spins which were not encoded because they were out of the tomographic plane are brought to the selected slice and start to contribute to the NMR signal. Since these spins are not phase encoded they will have a constant phase and their signal after the first FT will not be modulated. In other words their spatial position in the phase encoding direction have not been codified. Therefore after the second FT the signal corresponding to these spins appears as a line in the centre of the image where the frequency is equal to zero.

This problem was solved by modifying the spin-echo pulse sequence program. The sequence was modified to acquire every other spin-echo signal with a $+90^{\circ}$ pulse interspersed with a spin-echo signal acquired with a $-90^{\circ}$ pulse. To compensate from the signal inversion the receiver gain multiplied every echo from the $-90^{\circ}$ pulse by -1 and the echo from the $+90^{\circ}$ pulse by +1 . Therefore, at the end all spin-echo signals are the same as that of a normal spin-echo sequence. The pulse and gain changing affects only the signal from the $180^{\circ} \mathrm{RF}$ pulse. Since the $180^{\circ}$ pulse is always the same, the signal from the spins that were not encoded are also the same, but now they are modulated by the receiver gain. The modulation frequency produced by the receiver gain in the signal from the spins that are not phase encoded occurs at the Nyquist frequency for the second FT. The resulting effect of this modulation is to bring the artifact line from the centre of the image to the border of the field of view.

\subsection{Conclusion}

The relevant basic concepts of NMR necessary for the electrical current measurement have been reviewed, although this is not a complete review, since many aspects such as coil design, chemical shift etc. have not been covered.

The implemented Fourier transform algorithm worked satisfactorily and was used to analyse the experiments described in this thesis. The only problem found is that the actual image moves within the Field of View (FOV), depending on the slice position 
selected, but this is a particular problem of the NMR Bruker system. The reason for the movement is that the Bruker system uses the same RF pulse frequency to select the slice position, and as the reference frequency $\left(S_{\text {ref }}\right.$ and $S_{\text {ref2 }}$ in figure 2.21) to demodulate the NMR signal, thus producing an offset in the Fourier domain.

The phase correction algorithm also worked as expected producing reasonably flat phase images. This correction could be improved by the use of a higher order equation, instead of a simple linear one to correct the phase according to the pixel position.

With respect to the line artifact due to the Bruker MSVE sequence, the solution implemented was probably the simplest one since it did not require major modifications in the routine already implemented. Most of the images presented in this thesis, do not show the artifact line at the edge of the FOV because they may have been zoomed in, or the line may have been removed to avoid problems with the image contrast. 


\section{Chapter 3}

\section{Imaging of Electrical Current Distribution}

Imaging of electrical current distribution by NMR is a technique that makes it possible to indirectly analyse the electrical current distribution in any non uniform and conductive medium that can be imaged by NMR. This technique was first demonstrated as being able to measure the two-dimension current distribution in a cylindrical phantom by Joy et al. (1989). Scott et al. (1991) proved that it was also possible to measure a three-dimensional non uniform current distribution using a cubic phantom containing a ping-pong ball inside of it.

The basic principle is that NMR, being sensitive to any magnetic field, is able to measure the induced magnetic field produced by an electrical current applied through the object during the scan. Therefore, by measuring the three orthogonal components of the induced magnetic field it is possible to calculate the electrical current distribution in the NMR tomographic plane using for example the curl operator.

Although this technique is the only existing method of measuring electrical current inside of an object without interfering with the current distribution or causing damage to the medium being probed, it has some drawbacks which are inherent to the technique itself. The major disadvantage is that it uses pulses of DC current, and due to the intrinsic NMR noise a reasonably large current must be applied to the object. Pairs of symmetrical current pulses are usually applied, avoiding any problems of electrolysis that may occur but the current can produce physiological stimulation and is a limitation for in vivo studies. Another disadvantage, is that in order to measure the three orthogonal components of the current induced magnetic field, the object needs to be rotated inside the NMR magnet. The need to rotate and the diameter of the magnet bore limit the size of the objects that can be studied.

In a literature review only three abstracts about the application of this technique to measure electrical current in tissues were found. The first abstract showed images of the electrical current distribution in the chest of a sacrificed rabbit. The aim of this was to prove that the technique was able to measure electrical current in tissues (Thompson et al., 1991). The second abstract showed images of electrical current in a finger and the 
aim was to remove the error arising from the muscle motion caused by the current stimulation (Yip, et al. 1992). The authors claimed that their technique removed the motion artifact caused by the current stimulation, but the scan time was four times longer. The third abstract showed images of the current distribution in a mouse tumour, with the aim of understanding the electrical current distribution used in therapy (Zhao, et al., 1993). In the first two papers the electrical current applied was $41 \mathrm{~mA}$ and $6.25 \mathrm{~mA}$ respectively and in the tumour experiment $29.9 \mathrm{~mA}$.

To overcome the disadvantage of having to rotate the object, an alternating current (AC) technique was demonstrated by Scott et al. in 1992. The AC technique used RF currents at the Larmor precession frequency (around $100 \mathrm{MHz}$ for the $2.4 \mathrm{~T}$ NMR system). In a very simple approach, instead of rotating the object the nuclear-spins are rotated by the electrical current applied during the experiment. Tissue images using the AC technique at $100 \mathrm{MHz}$ were recently published (Scott et al., 1995). Unfortunately at $100 \mathrm{MHz}$ frequency the electrical current will propagate straight through the cell membranes, so the resulting current distribution will not be the same as in EIT which uses an applied current at a frequency of $50 \mathrm{kHz}$.

In a very recent paper (Ider et al., 1995), a theoretical study and simulation of a technique which may be able to measure electrical current at low frequency was reported. The authors claimed that this technique should be able to measure AC electrical current in the $\mathrm{KHz}$ range, though this remains to be proven experimentally.

In conclusion therefore, considering the aim of this thesis, which was to study the electrical current propagation in the head at low frequency, the suitable technique is still thought to be the DC current technique.

This chapter describes the version of the DC technique developed at UCL, and which is based on the technique first published by Joy et al.(1989) and improved by Scott et al. (1991). The major difference with the UCL implementation is that we compute the absolute current per pixel instead of current density per pixel.

\section{Imaging of Electrical Current Distribution}

The process of computing an image of current distribution for each orthogonal component $I_{x}, I_{y}$ and $I_{z}$ in a specific tomographic plane involves a sequence of five steps: 1- The NMR experiment with applied electrical current pulses;

2- Determination of the phase shifts produced by the current; 
3- Phase unwrapping;

4- Electrical current image calculation;

5- Image registration.

\subsection{The NMR experiment with applied electrical current pulses}

To perform the experiment to measure the electrical current distribution, a sequence of current pulses, synchronised with the NMR pulses must be applied to the object being imaged. Section 2.2.3 describes the NMR spin-echo sequence used in the experiments and figure 3.1 illustrates such a sequence of current pulses synchronised with the NMR pulse sequence.

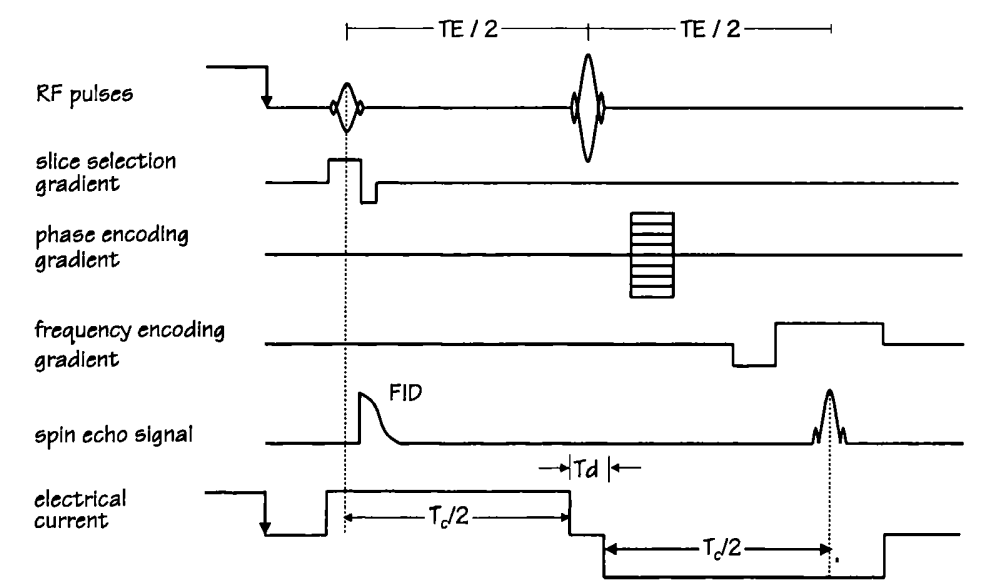

Figure 3.1 - The NMR spin-echo sequence and the electrical current pulses.

The electrical current applied to the object during the process of NMR data acquisition is divided into a positive current period and a negative current period. The positive current is switched on just before the $90^{\circ} \mathrm{RF}$ pulse and lasts until just before the $180^{\circ} \mathrm{RF}$ pulse. After the refocusing pulse a negative pulse of current is applied until the NMR echo signal is acquired in the presence of the frequency encoding gradient.

The total period that the current is applied is equal to $T_{c}$ and the period during which the current is turned off for the spin inversion is equal to $T_{d}$. If the $90^{\circ} \mathrm{RF}$ pulse and $T_{d}$ interval have the same duration the total current time will be equal to the echo time $T E$.

A detailed explanation of the current source, the current pulse and the NMR trigger control is given in section Appendix C. 


\subsection{Determination of the phase shift caused by the current}

\section{The current magnetic field and phase shift of the nuclear spins}

The NMR signal $S\left(t_{x}, t_{y}\right)$ for a slice parallel to the $x y$-plane in the time domain is given by,

$$
S\left(t_{x}, t_{y}\right)=\int_{x} \int_{y} \rho(x, y) e^{i \gamma\left(x G_{x}+y G_{y}\right)} d x d y
$$

where $\rho(x, y)$ is the nuclear spin density, $G_{x}$ is the gradient field in the $x$-direction, $G_{y}$ is the gradient field in the $y$-direction.

When an electric current is applied to an object with electrodes that are small with respect to the object size, the current will spread through the object and will produce a current magnetic field $\boldsymbol{B}_{\boldsymbol{c}}(\boldsymbol{x}, \boldsymbol{y}, \boldsymbol{z})$. Consider that the electric current is applied in the $y$-direction and the main field $\boldsymbol{B}_{0}$ is in the $z$-direction. The new current magnetic field $\boldsymbol{B}_{\boldsymbol{c}}(\boldsymbol{x}, \boldsymbol{y}, \boldsymbol{z})$ may be incorporated into equation (3.1) as follows,

$$
S\left(t_{x}, t_{y}\right)=\int_{x} \int_{y} \rho(x, y) e^{i \gamma\left(x G_{x} t_{x}+y G_{y} t_{y}\right)} e^{i \gamma B_{c}(x, y, z) T_{c}} d x d y
$$

The two-dimensional spin density $\rho(x, y)$ may be represented by its Fourier transform $F\left(k_{x}, k_{y}\right)$ where $k_{x}$ and $k_{y}$ are the wavenumbers in the $x$ - and $y$-direction respectively,

$$
F\left(k_{x}, k_{y}\right)=\int_{-\infty}^{+\infty} \int_{-\infty}^{+\infty} \rho(x, y) e^{-i\left(x k_{x}+y k_{y}\right)} d x d y
$$

Comparing equations (3.2) and (3.3) as before (section 2.2.2) we see that

$$
\begin{array}{ll}
x k_{x}=\gamma x G_{x} t_{x}+\gamma B_{c}(x, y, z) T_{c} & t_{y}<t<t_{x} \\
y k_{y}=\gamma y G_{y} t_{y}+\gamma B_{c}(x, y, z) T_{c} & 0<t<t_{y}
\end{array}
$$

where the components $\gamma x G_{x}$ and $\gamma y G_{y}$ are the frequency components produced by the phase and frequency encode magnetic gradients respectively. Together they define a point in the $x y$-plane for the image reconstruction procedure. The other component $\gamma B_{c}(x, y, z)$ is the frequency determined by the current induced magnetic field.

In equation (3.4) the factor $\gamma B_{c}(x, y, z)$ must be smaller than $\gamma x G_{x}$ and $\gamma y G_{y}$ otherwise it may interfere with the phase and frequency encode processes which would result in pixel movement and produce images artifacts. 
To estimate the size of the frequency shift due to the readout gradient and that caused by the current magnetic field, consider two adjacent pixels. We will use the same parameters as for the phantom image shown in the last chapter, i.e., FOV equal to 10 $\mathrm{cm}$ and sample rate $25 \mathrm{KHz}$. For a quadrature detector, the complex FT gives a spectrum bandwidth $^{10}$ which is equal to the sample rate, i.e. $25 \mathrm{kHz}$. Thus the read gradient is equal to $2500 \mathrm{~Hz} \mathrm{~cm}^{-1}(25000 \mathrm{~Hz} / 10 \mathrm{~cm})$, or $0.936 \mathrm{mTm}^{-1}\left(2500 \mathrm{~Hz} \mathrm{~cm}^{-1}\right.$ divided by $\gamma=2.67$ $\left.\mathrm{x} 10^{8} \mathrm{~T}^{-1} \mathrm{~s}^{-1}\right)$. The image matrix is equal to 256 by 256 pixels and the pixel size is 0.39 $\mathrm{mm}(100 \mathrm{~mm} / 256)$. Thus considering the figure of $2500 \mathrm{~Hz} \mathrm{~cm}^{-1}$, the difference in frequency between two adjacent pixels is equal to $97.65 \mathrm{~Hz}$.

The magnetic field of the electrical current has to produce a frequency shift smaller than $97.47 \mathrm{~Hz}$ (or $365 \mathrm{nT}$ ), otherwise the image would be destroyed. If we consider that the current propagates in the centre of one pixel, from Biot-Savart Law $B=\left(I \mu_{0}\right) /(2 \pi \rho)$, ( $\rho$ is the distance from the centre of one pixel and the centre of its next neighbour), it would require a current of:

$$
I=\frac{2 \pi \rho B_{C}}{\mu_{0}}=\frac{2 \pi 36510^{-9}\left[\frac{\mathrm{Wb}}{\mathrm{m}^{2}}\right] 0.3910^{-3}[\mathrm{~m}]}{4 \pi 10^{-7}\left[\frac{\mathrm{Wb}}{\mathrm{m} \mathrm{A}}\right]}=0.71 \mathrm{~mA}
$$

to produce a frequency shift equal to the frequency encoding gradient in the neighbourhood of the current pixel. This situation is very unlikely to happen since, as we are going to see, the maximum current per pixel measured in the experiments was around $100 \mu \mathrm{A} /$ pixel. Added to this fact, we never have an isolated pixel conducting electrical current, but a region of the object through which the current spreads. This means that the field produced by each pixel is reduced by the field produced by its neighbouring pixel as happens in the centre of a conductor where the field intensity is zero.

A further point to be considered relates to the components of the current magnetic field. Since the electric current is applied in the $y$-direction, the current induced magnetic field is in a plane parallel to the $x z$-plane. From the Larmor equation the nuclear precession frequency is proportional to the magnetic field strength. Therefore resolved frequencies, and not the difference between the frequencies where the signal intensity decays $3 \mathrm{~dB}$. 
to produce effects on the spin precession it is necessary to change the main field $\boldsymbol{B}_{\boldsymbol{0}}$. Hence, only the current induced magnetic field components which are parallel to the main field will produce variations in the frequency of precession.

Since the change in the frequency of precession produced by the current magnetic field is very small, it will just appear as a phase shift in the precession frequency which is given by,

$$
\varphi_{c} \underset{B_{d} / z}{\mid}=\left.\int_{0}^{T_{c}} \gamma B_{c}(x, y, z)\right|_{z} d t=\left.\gamma B_{c}(x, y, z)\right|_{z} T_{c}
$$

\section{The phase shift produced by the main field inhomogeneity}

Inhomogeneities in the main field $\boldsymbol{B}_{0}$ will produce small phase shifts across the image $^{11}$. These small phase shifts will be mixed with those arising from the magnetic field induced by the current. Since for current image distribution calculation it is necessary to know exactly the phase produced just by the current, a method of compensation for the $\boldsymbol{B}_{\boldsymbol{0}}$ inhomogeneities must be used.

Basically there are two procedures to avoid the phase shifts caused by field imperfections: 1) acquire an image with the current on and another without the current and then subtract the two. In this way, the phase shifts caused by the main field imperfections will be cancelled; 2) acquire an image with two different current pulses sequences, first a phase image with positive-negative current pulse sequence, second a phase image with a negative-positive current pulse sequence and then subtract the images. The second procedure is the one suggested by Joy et al. (1989).

In figure 3.2(a), vector $\boldsymbol{M}$ represents the bulk magnetisation of a certain volume $v$ in the sample after the $90^{\circ} \mathrm{RF}$ pulse. Consider that no current is applied through the object and at this point $v$ there is a $\boldsymbol{B}_{0}$ inhomogeneity which produces a phase shift $\varphi_{1}$ on the net magnetisation. After the $180^{\circ}$ refocusing pulse the total phase shift will be:

$$
\varphi_{n}=\left(180-\varphi_{1}\right)
$$

Now consider the situation where a positive-negative current pulse sequence, as shown in figure 3.1 , is applied to the object. The orientation of the spins for this 


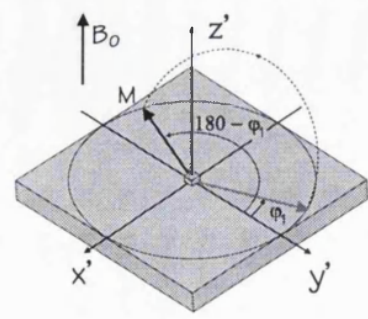

(a)

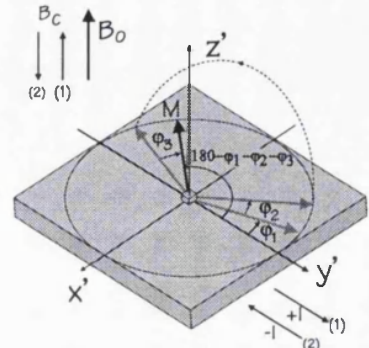

(b)

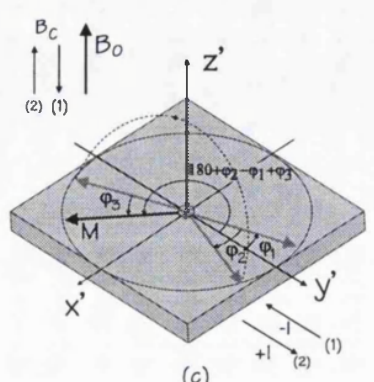

(c)

Figure 3.2-The net magnetisation in the rotating frame.(a)The phase shift produced by main field inhomogeneities;(b) and (c)The phase shifts produced by a PN and NP current pulse sequence, respectively.

situation are represented in figure 3.2(b). The same phase shift $\varphi_{I}$ caused by the main field imperfection is present. Consider also that the positive current pulse, applied in the $+y$-direction (number 1 in figure $3.2($ b)), produces a small increment in the main field $\boldsymbol{B}_{0}$. This $\boldsymbol{B}_{0}$ increment, by the Larmor equation, produces a slight increase in the frequency of precession which gives rise to a phase shift $\varphi_{2}$. Then the current is turned off for the spin inversion (figures 3.1) and a negative current pulse in the -y-direction is then applied (number 2 in figure 3.2(b)). This produces a decrease of the main field $\boldsymbol{B}_{0}$ at the same point $v$ which decreases the frequency of precession and produces a phase shift $\varphi_{3}$. The total phase shift $\varphi_{t \pm}$ produced by applying the positive-negative current pulse sequence is thus equal to:

$$
\varphi_{1 \pm}=\left(180-\left(\varphi_{1}+\varphi_{2}+\varphi_{3}\right)\right)
$$

Subtracting the image with the positive-negative current pulse from the image with no current applied, i.e., equations (3.8) and (3.7), the total phase shift $\varphi_{t \pm n}$ is,

$$
\varphi_{t \pm n}=180-\varphi_{1}-\varphi_{2}-\varphi_{3}-180+\varphi_{1}=-\left(\varphi_{2}+\varphi_{3}\right)
$$

which is just the phase shift produced by the current magnetic field.

For the second procedure, first an image with a positive-negative current sequence is acquired. The phase shift produced by this current pulse sequence, is the same as in figure 3.2(b) (equation (3.8)). After that a second image now with a negativepositive pulse sequence is acquired. This sequence is illustrated in figure 3.2(c).

In figure $3.2(\mathrm{c})$ the same phase shift $\varphi_{\text {I }}$ produced by the main field imperfection is still present. During the $90^{\circ} \mathrm{RF}$ pulse, a negative current pulse is applied to the object 
and this will produce a negative current magnetic field which will decrease the frequency of precession and produces a phase shift $\varphi_{2}$ (figure 3.2(c)). After that comes the $180^{\circ} \mathrm{RF}$ pulse during which the spins are rotated and for this period the current is turned off (figure 3.1). Following this, the current is reversed which increments the frequency of precession producing the phase shift $\varphi_{3}$.

The total phase shift $\varphi_{t \mp}$ produced by the negative-positive current sequence after the refocusing pulse, is

$$
\varphi_{t \mp}=180+\left(\varphi_{2}-\varphi_{1}\right)+\varphi_{3}
$$

Calculating the difference between the images with positive-negative current and negative-positive current, i.e. equation (3.8) and equation (3.10), the total phase shift $\varphi_{t \pm \neq}$ is,

$$
\begin{aligned}
& \varphi_{t \pm f}=180-\varphi_{1}-\varphi_{2}-\varphi_{3}-180-\varphi_{2}+\varphi_{1}-\varphi_{3} \\
& \varphi_{t \pm f}=-2\left(\varphi_{2}+\varphi_{3}\right)
\end{aligned}
$$

Both procedures eliminate the phase produced by main field inhomogeneity across the image, but in the second one the current induced phase shift is twice that produce by the first. From equation (3.6) the current magnetic field can be determined from the phase shift difference. For the first case (i.e., the difference between one image with current and another with no current) the current magnetic field is equal to,

$$
B_{C}=\frac{\varphi_{t \pm n}}{\gamma T_{C}}
$$

and for the second case (difference between one image with a positive-negative and another with a negative-positive sequence) the current magnetic field is equal to,

\subsection{Phase "unwrapping"}

$$
B_{c}=\frac{\varphi_{t+f}}{2 \gamma T_{C}}
$$

In Magnetic Resonance Imaging, the process of image reconstruction is through the Fourier Transform of the spin-echo signal. The Fourier Transform produces a complex function, and the argument of this produces what we call the NMR phase image.

The arguments of a phase image are computed by the trigonometric arctangent 
function. The arctangent is limited to the prime intervals $+\pi$ and $-\pi$, which are called the principal values, any argument outside this interval will be reflected back within these limits. This "wrapping" effect produces an image with a sequence of black and white gradations, instead of a unique smooth transition from black to white. Figure 3.3(a), illustrates what a phase wrapped image looks like and figure 3.3(b) is an intensity profile along a horizontal line one pixel wide across the centre of the image. Each discontinuity from white to black characterises a $2 \pi$ transition, as can be seen on the profile.

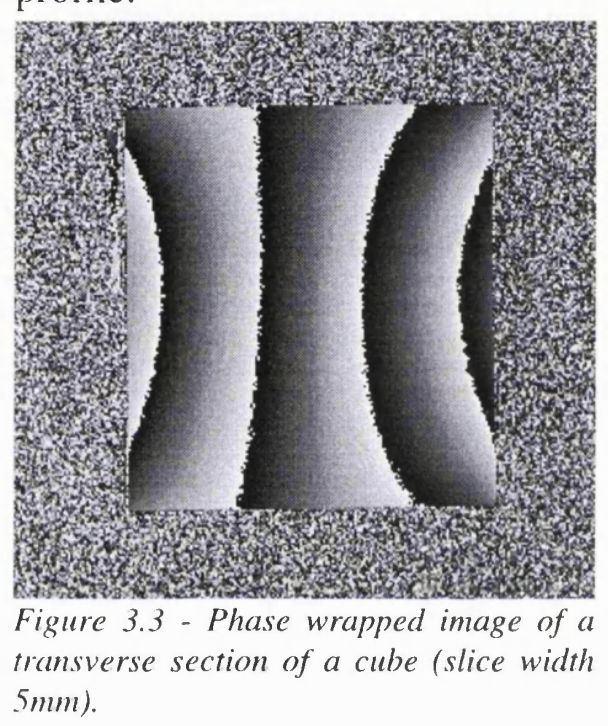

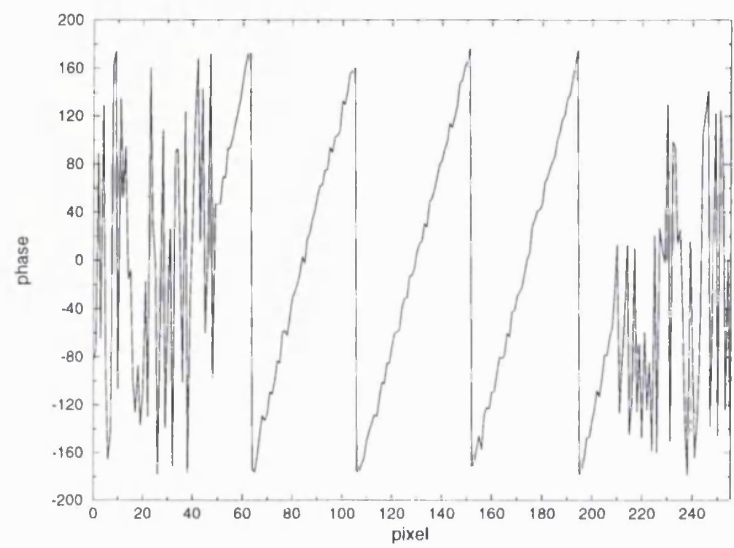

Figure 3.4 - Phase wrapped image profile along a horizontal centre line of figure 3.3 .

Phase unwrapping consists of computing the actual phase from the principal values of a sequence of arguments. Many techniques for this purpose have been published: differentiation, wrapping and integration the principal values into each path (Itoh, 1982; Hedley and Rosenfeld, 1992); cellular automata (Ghiglia et al., 1987; Spik, 1991) and least squares approximation (Lin and Vesecky, 1992).

\subsubsection{Phase unwrapping: Basic principles}

Consider a sequence of arguments, represented by $\varphi_{p v}(n)$, where the label $p v$ means principal value. These principal values are the NMR imaging data, as illustrated in figure 3.3 , and the limits for these values are the prime interval $+\pi-\pi$.

$$
-\pi \leq \varphi_{p v}(n) \leq+\pi
$$

Suppose that $\varphi_{t}(n)$, is the $n^{\text {th }}$ true phase value of this array, which is recovered from the principal values. A wrapping operator $\omega$ can be defined as, 


$$
\omega_{i}\left[\varphi_{t}(n)\right]=\varphi_{t}(n)+2 \pi k_{i}(n) \quad \therefore-\pi \leq \omega_{i}\left[\varphi_{t}(n)\right] \leq+\pi
$$

where $k \in \Re$ integers and $i$ is a label to specify the wrapping operator. All this operator does is to find out how many $2 \pi$ intervals it is necessary to add or subtract from the actual phase $\varphi_{t}$, to get to the principal values. The difference between two principal values using the wrapping operator $\omega_{i}$, is given by,

$$
\begin{aligned}
\omega_{1}\left[\varphi_{t}(n)\right]-\omega_{1}\left[\varphi_{t}(n-1)\right] & =\varphi_{t}(n)+2 \pi k_{1}(n)-\left[\varphi_{t}(n-1)+2 \pi k_{1}(n-1)\right] \\
& =\varphi_{t}(n)-\varphi_{t}(n-1)+2 \pi\left[k_{1}(n)-k_{1}(n-1)\right]
\end{aligned}
$$

Applying the wrapping operator to the difference,

$$
\begin{aligned}
\omega_{2}\left[\omega_{1}\left[\varphi_{t}(n)\right]-\omega_{1}\left[\varphi_{t}(n-1)\right]\right] & =\varphi_{t}(n)-\varphi_{t}(n-1)+ \\
& +2 \pi\left[k_{1}(n)-k_{1}(n-1)\right]+2 \pi k_{2}(n)
\end{aligned}
$$

considering the wrapping operator definition,

$$
-\pi \leq \omega_{2}\left[\omega_{1}\left[\varphi_{t}(n)\right]-\omega_{1}\left[\varphi_{t}(n-1)\right]\right] \leq+\pi
$$

and since the NMR data are within the range $+\pi$ and $-\pi$,

$$
-\pi \leq \varphi_{t}(n)-\varphi_{t}(n-1) \leq+\pi
$$

thus,

$$
2 \pi\left[k_{1}(n)-k_{1}(n-1)\right]+2 \pi k_{2}(n)=0
$$

and

$$
\begin{aligned}
& \varphi_{t}(n)-\varphi_{t}(n-1)=\omega_{2}\left[\omega_{1}\left[\varphi_{t}(n)\right]-\omega_{1}\left[\varphi_{t}(n-1)\right]\right] \\
& \varphi_{t}(n)=\varphi_{t}(n-1)+\omega_{2}\left[\omega_{1}\left[\varphi_{t}(n)\right]-\omega_{1}\left[\varphi_{t}(n-1)\right]\right]
\end{aligned}
$$

Starting at one corner of the image and assigning to the initial pixel a true phase, the path of a sequence of principal values can be unwrapped up to the $n^{\text {th }}$ pixel.

$$
\begin{aligned}
& n=1 \\
& \varphi_{t}(0)=\varphi_{p v}(0) \\
& \varphi_{t}(1)=\varphi_{t}(0)+\omega_{2}\left[\omega_{1}\left[\varphi_{t}(1)\right]-\omega_{1}\left[\varphi_{t}(0)\right]\right] \\
& \varphi_{t}(2)=\varphi_{t}(1)+\omega_{3}\left[\omega_{2}\left[\varphi_{t}(2)\right]-\omega_{2}\left[\varphi_{t}(1)\right]\right] \\
& \varphi_{t}(3)=\varphi_{t}(2)+\omega_{4}\left[\omega_{3}\left[\varphi_{t}(3)\right]-\omega_{2}\left[\varphi_{t}(2)\right]\right] \\
& \cdot \\
& \cdot \\
& \varphi_{t}(n)=\varphi_{p v}(0)+\sum_{n=1}^{m} \omega_{n}\left[\omega_{n-1}\left[\omega_{t}(n)\right]-\omega_{n-1}\left[\omega_{t}(n-1)\right]\right]
\end{aligned}
$$


Equation (3.22), originally developed by Itoh(1982), states that a sequence of three operations are necessary to unwrap an image: 1) determine the wrap difference between the starting point and the next phase point, 2) wrap the difference, so that it will be a prime interval and, finally, 3) add it to its starting point. By repeating these three steps along each path, the whole image will be unwrapped.

For NMR phase images all pixels are principal values since the matrix is computed from the arctangent function. This means that it is not necessary to wrap the pixel values before computing the difference between two neighbours. On the other hand, the difference is out of the principal value range when a transition of $2 \pi$ has occurred. Every time it occurs the phase difference is wrapped and added to the value of the last unwrapped pixel. To implement this procedure two thresholds are necessary, one of $\pi$, for positive difference angles, and another of $-\pi$, for negative difference angles. Once the difference is greater than $+\pi, 2 \pi$ is either subtracted from the difference, or $2 \pi$ is added to it.

Another possibility, avoiding the need for a threshold is to compute the phase difference by vectorial algebra.

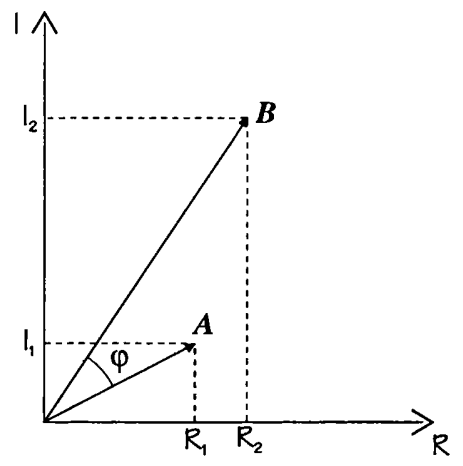

Figure 3.5 - The difference angle between two complex vectors

As shown in figure 3.5 , the phase difference $\varphi_{d}$ between two complex vectors can be computed by dividing the scalar and vectorial products, equation (3.23).

$$
\frac{\boldsymbol{A} \times \boldsymbol{B}}{\boldsymbol{A} \cdot \boldsymbol{B}}=\frac{|\boldsymbol{A}||\boldsymbol{B}| \sin \left(\varphi_{d}\right)}{|\boldsymbol{A}||\boldsymbol{B}| \cos \left(\varphi_{d}\right)}=\tan \left(\varphi_{d}\right)
$$

each product is calculated by the equations,

$$
\boldsymbol{A} \times \boldsymbol{B}=\left(R_{1} I_{2}-R_{2} I_{1}\right) \hat{\boldsymbol{a}}_{\boldsymbol{k}} \text { and } \boldsymbol{A} \cdot \boldsymbol{B}=R_{1} R_{2}+I_{1} I_{2}
$$

in this way, the phase difference is calculated directly from the real and imaginary 
components of the FT, equation (3.25)

$$
\tan \left(\varphi_{d}\right)=\frac{R_{1} I_{2}-I_{1} R_{2}}{R_{1} R_{2}+I_{1} I_{2}}
$$

This procedure avoids the need to define a threshold to wrap the difference before the integration (Patel and Hu, 1993).

\subsubsection{Noise artifacts}

Whatever the method used to compute the difference between phases before the integration process, if for any reason noise is present in the path, a $2 \pi$ interval can be added to the path producing artifacts on the final image. In figure 3.6 some artifacts produced by noise points after unwrapping are illustrate.

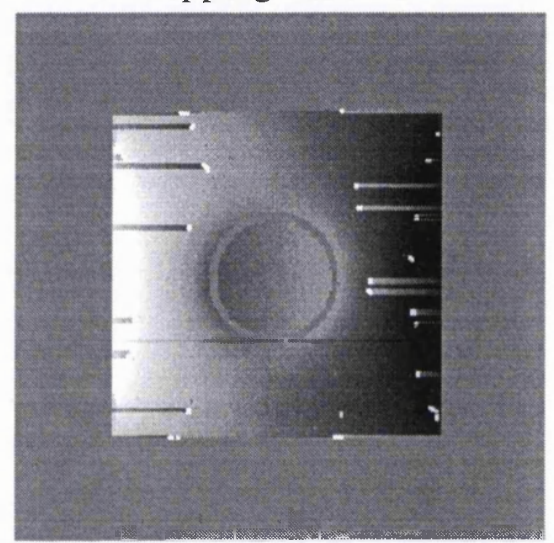

Figure 3.6- Artifacts produced during the process of phase unwrapping.

A routine to detect the presence of noise is based on checking all $2 \times 2$ sample regions in the two-dimensional principal-values array, figure 3.7. Basically, the sum of the wrapped difference along the closed path is determined. If the sum is different from zero, equation (3.19) is violated and all points are flagged as inconsistent and are not used during the unwrapping process, otherwise all points are said to be consistent (Hedley and Rosenfeld, 1992; Ghiglia et al., 1987).

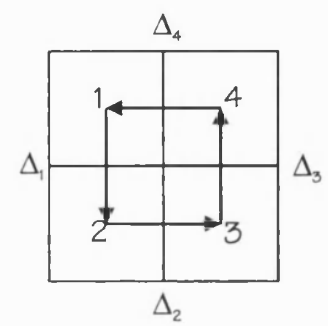

$$
\sum_{k=1}^{4} \omega[\Delta k] \begin{cases}=0, & \text { consistent path } \\ \neq 0, & \text { inconsistent path }\end{cases}
$$

Figure 3.7 . The region of $2 \times 2$ pixels and the closed path used to detect noise during the phase unwrapping process.

In figure 3.8, some pixels flagged as inconsistent are illustrated and highlighted 
in white. All these regions are jumped during the phase unwrapping process, avoiding the artifacts produced on figure 3.6. Figure 3.9 illustrates the unwrapped image with the flagged points.

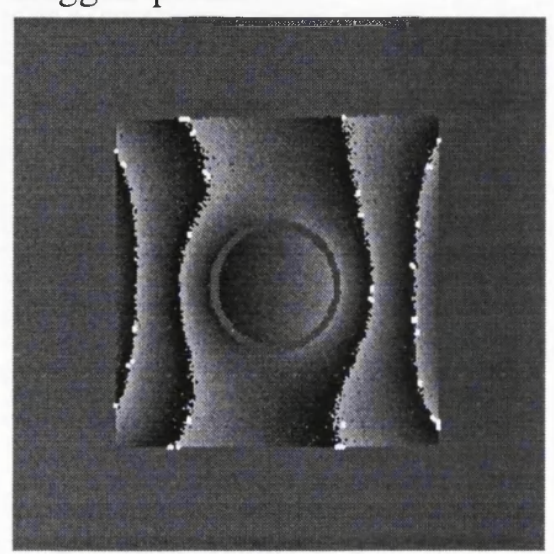

Figure 3.8 - Highlighted noise pixels detected by closed path difference checking.

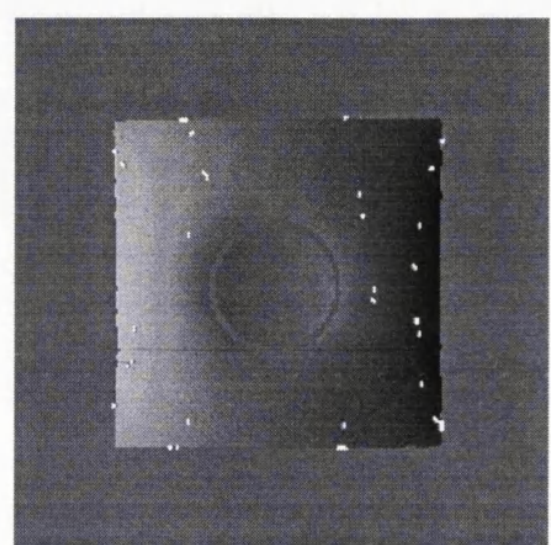

Figure 3.9 - The flagged points after the phase image in figure 3.8 had been unwrapped.

Another problem is related to the field of view (FOV). On NMR images, as illustrated in figure 3.3, the FOV is greater than the object that has been imaged. This introduces a dilemma about whether a pixel is inside or outside the object. One possible solution to this problem is to look at the magnitude image, if its value is lower than a threshold, the pixel is outside, otherwise it is inside (Hedley and Rosenfeld, 1992). This technique can also be used to avoid very low signal pixels which introduce artifacts in the unwrapped image.

\subsubsection{The implemented algorithm}

Phase unwrapping is a process that does not always work by itself the first time. It is very dependent on the SNR of the image, for instance with a high and uniform SNR the process of phase unwrapping is straight forward, otherwise many artifacts will appear in the unwrapped image. In this case the process needs to be repeated many times with a constant intervention to change noise pixel values that were unable to be detected.

An algorithm to unwrap the NMR phase images was written specifically for this project. It was implemented in the " $\mathrm{C}$ " programming language and is divided into four main routines: image thresholding, noise detection, phase unwrapping, and phase interpolation. 


\section{Image thresholding}

Image thresholding consists of defining a threshold value to distinguish the background pixels from the objects pixels in the FOV of the NMR magnitude image. The threshold algorithm is divided into three steps: image filtration, histogram calculation and threshold calculation.

- image filtration : In the applied current experiment, due to the current-spin time integration, a long echo-time is required. Therefore, mainly in the case of the piglet experiments where no control over the relaxation times exists, the NMR images have a poor SNR. During the implementation of this algorithm we found that if the NMR magnitude image was slightly smoothed, the threshold definition was more effective. Hence before the threshold calculation, the magnitude image is filtered with a Butterworth filter. The filtering routine used was that from the UNC image processing package called "butter". The parameters used were $n=8$ (filter order) and $c=0.3$ (which defines the filter bandwidth). The UNC butter routine Fourier transforms, filters and makes the inverse Fourier transform of the magnitude image;

- histogram calculation: Once the NMR image is filtered the next step is to calculate the image histogram. The routine implemented finds the minimum and the maximum values of the image. This range is then divided into a hundred parts and the frequency of pixel values for each part is counted. In figure 3.10 is shown the histogram of the image shown in figure 3.11(d);

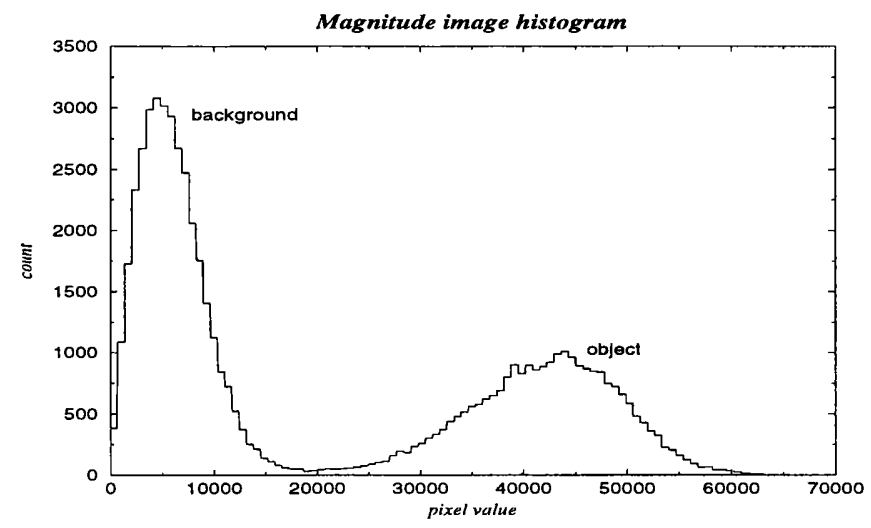

Figure 3.10 - Image magnitude histogram used to define the threshold value during the process of phase unwrapping.

- threshold calculation : The histogram in figure 3.10 has two well defined 
regions, one related to the background pixel noise values in the FOV and another related to the object pixels values in the FOV. The threshold routine looks for the minimum frequency between the two maximums. The pixel value corresponding to the minimum frequency is set as the threshold value between the two regions.

Finally, a mask image is generated with the corresponding pixels set to unity if the signal intensity is bigger than the threshold. This mask image defines the ROI that will be unwrapped by the recursive algorithm. The major problem in this step were found to be the regions of the skull in the piglet head images. These regions have a low signal and therefore tend in general to be set to zero, leaving gaps in the mask image. At present, the routine displays the mask image and these gaps are manually filled, otherwise they would not be considered by the unwrapping routine.

\section{Noise detection}

It is important to detect noise pixels before starting the process of phase unwrapping. If any pixels have a wrong phase value, artifacts will be produced in the final unwrapped image. Two techniques were implemented to exclude these pixels from the process of phase unwrapping: low signal intensity and inconsistent phase loops.

- Low signal intensity : This step uses the same threshold obtained from the image histogram to flag all the pixels within the ROI with a low signal level. These pixels are classified as potentially noise pixels and their corresponding phase are avoided during the process of phase unwrapping. In general the bone regions in the piglet images are detected but most of these regions are not noise at all. To reduce this problem the threshold is manually reduced up to the point where artifacts starts to appear in the unwrapped image;

- Inconsistent phase loops : In this step the actual phase value is checked in a loop of 2 by 2 pixels as previously described. If an inconsistent loop is found, the four pixels are flagged as phase inconsistent and their phase values are not used during the phase unwrapping. An interesting point is that most of the pixels that have a low signal intensity such as the regions of the bones, are not always detected as phase inconsistent and these pixels do not produce any artifact in the phase unwrapped image. On the other hand pixels with high signal intensity are sometimes classified as phase inconsistent and they certainly produce artifacts 
in the unwrapped image.

\section{Phase unwrapping}

To unwrap the phase image a recursive painting algorithm was implemented. Initially, we select a starting pixel for each isolated ROI inside the FOV and mark it as painted. The starting point must be selected in a region where the phase is noise free. Next, for each ROI the algorithm looks for the next neighbouring pixel that has not been flagged by the threshold or the loop technique. Once a neighbouring pixel is selected the program determines the difference between their phase values and wraps the difference, i.e., if the difference is bigger than $+\pi$ subtract $2 \pi$, if smaller than $-\pi$ add $2 \pi$. The unwrapped phase value of the neighbour is then made equal to the calculated wrapped phase difference plus the starting pixel phase value and it is set as painted. The algorithm then moves to this pixel and from it identifies its next neighbouring pixel that is neither flagged nor painted and repeats the above steps. The recursion takes place until all the pixels not flagged by the noise detection algorithm in the ROIs are painted.

\section{Phase interpolation}

After the unwrapping algorithm has been applied, the next step is to deal with all the noise flagged pixels in the unwrapped image. To treat these pixels a local linear interpolation is used. The interpolating subroutine looks for each pixel within the ROI that has been flagged, finds the next ten neighbouring pixels on either side and uses these pixel values to interpolate the centre flagged pixel.

In the case of the images acquired with no applied current, the interpolation process is very simple because the phase is linear. Actually in general the images with no current do not need to be unwrapped, but the algorithm was applied just to correct some noise pixels and remove the background noise in the FOV. This is not the case with the current images where the phase changes can have any shape and the process of interpolation is much more complicated. The local linear interpolation does not always work, and fitting polynomials is difficult because of the presence of noise. Therefore, after the local linear interpolation, the unwrapped phase is displayed and if the interpolated pixels do not show a good contrast with its neighbourhood pixels the phase value is manually corrected.

Figure 3.11 illustrates each step in the process of phase unwrapping. The image is a transverse section of a cube, described in section 5.1.1, in which a cylinder replaces 
the perspex platform.

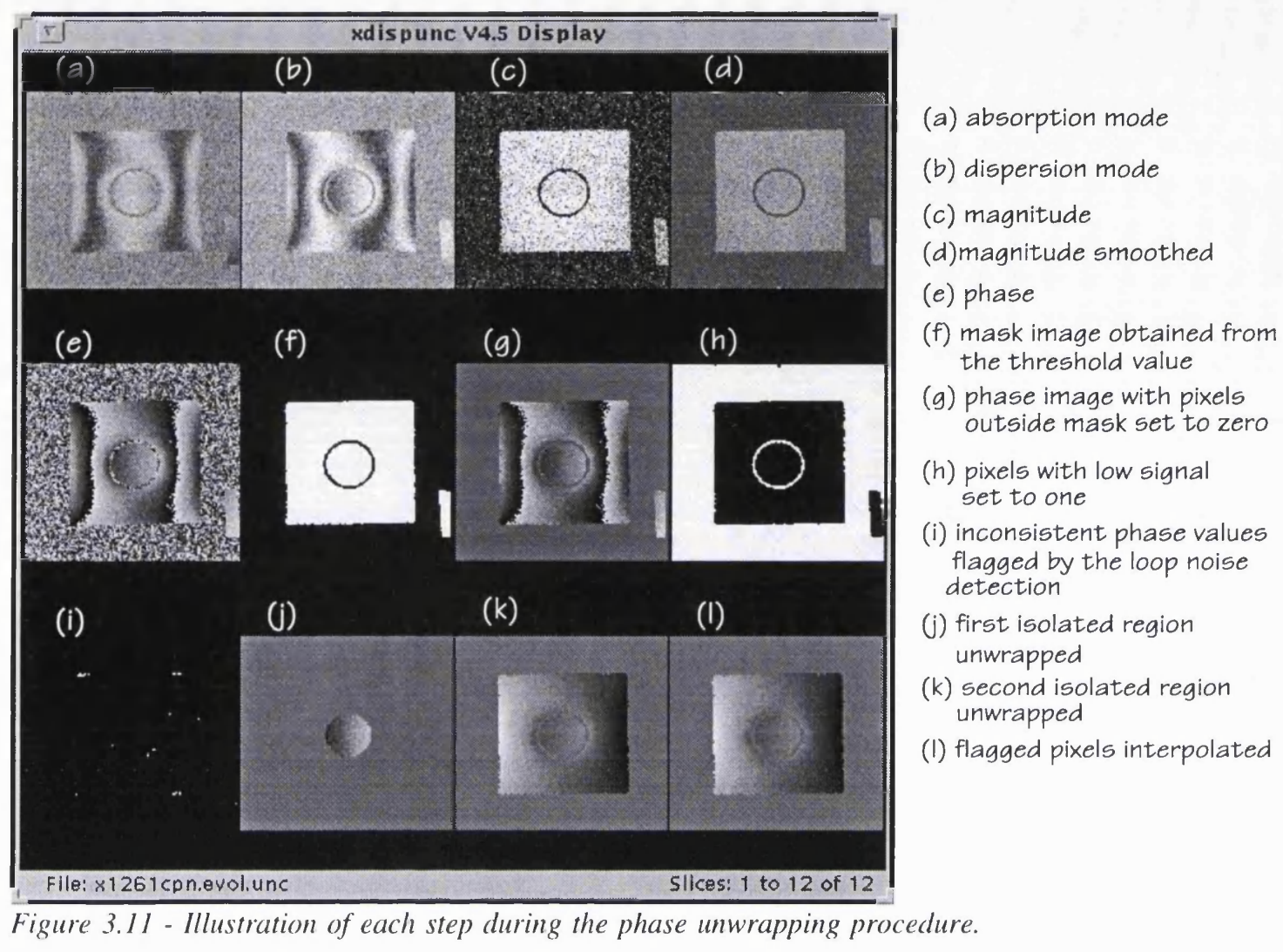

\subsection{Electrical Current Image Distribution Calculation}

\section{The Current Distribution and Ampere's Law}

According to the Biot-Savart Law, an electrical current produces a magnetic field in a plane orthogonal to the current direction and with an orientation given by the righthand rule. Hence a component of current in the $y$-direction produces a magnetic field parallel to the $x z$-plane. From Ampere's Law, the current through any path can be computed using the following equation,

$$
\oint \boldsymbol{H} \cdot d \boldsymbol{L}=\frac{1}{\mu_{0}} \oint \boldsymbol{B}_{C} \cdot d \boldsymbol{L}=\boldsymbol{I}
$$

where, $\boldsymbol{B}_{C}$ is the current induced magnetic flux density (Tesla or $\mathrm{Wb} / \mathrm{m}^{2}$ ), $\boldsymbol{H}_{C}$ is the current magnetic flux intensity $(\mathrm{A} / \mathrm{m}), \mu_{0}$ is the permeability of free space, equal to 4 $\pi 10^{-7} \mathrm{H} / \mathrm{m}$. Therefore, if it is possible to measure at any region the magnetic field produce by an electrical current, it is also possible to compute the current through this region.

By the NMR technique it is possible to compute an image where the pixel values 
are the magnetic field density produced by an electrical current passing through the object. Considering that $\boldsymbol{B}_{c}=\mu_{0} \boldsymbol{H}_{c}$ and equation (3.12) the current induced magnetic field intensity is equal to,

$$
H_{C}=\frac{\varphi_{C \pm / \mp}}{\gamma T_{C} \mu_{0}}
$$

The limitation is that $\varphi_{C}$ is the nuclear spin phase shift produced only by the component of current magnetic field parallel to the main field $\boldsymbol{B}_{\boldsymbol{0}}$, since just this component affects $\boldsymbol{B}_{0}$. Therefore the value of $\boldsymbol{H}_{C}$ calculated by equation (3.27) is just one component of the current induced magnetic field, which is in the direction of $\boldsymbol{B}_{0}$. To measure the other components the object needs to be rotated.

Also, equation (3.26) is an internal product, which means that the value of $\boldsymbol{H}_{\boldsymbol{C}}$ is the projection in the direction of $d \boldsymbol{L}$. But if a square path is chosen and the object is rotated by $90^{\circ}$ the direction of $d \boldsymbol{L}$ will match the direction of $\boldsymbol{B}_{\boldsymbol{0}}$. In this situation it is the projection of $\boldsymbol{H}_{C}$ along of the path that is measured directly by the NMR experiment and the cosine factor of the dot product can be dropped.

Since to create the closed paths requires rotation of the object and a different sequence of NMR image slices, the calculation of each component is analysed separately below. In the following analyses consider that the desired current tomographic plane is parallel to the $x z$-plane.

\section{Iy Current Calculation}

To compute the $I_{y}$ current component, two sets of images are required. One with the $x$-axis parallel to the main field and another with $z$-axis parallel to the main field. Each set is composed of two images, one obtained with a positive-negative current pulse sequence and another without current or using the difference current pulse sequences, described in section 3.2 .

The current distribution in the tomographic plane is computed pixel by pixel. This is done by selecting regions of 3 by 3 pixels, as illustrated in figure 3.12. The $\oint \boldsymbol{H} \cdot d \boldsymbol{L}$ is computed along the closed path 1-2-3-4-5-6-7-8-1, which is equal to,

$$
I_{y}=\oint \boldsymbol{H} \cdot d \boldsymbol{L}=\int_{1}^{3} H_{13} d L_{13}+\int_{3}^{5} H_{35} d L_{35}+\int_{5}^{7} H_{57} d L_{57}+\int_{7}^{1} H_{71} d L_{71}
$$



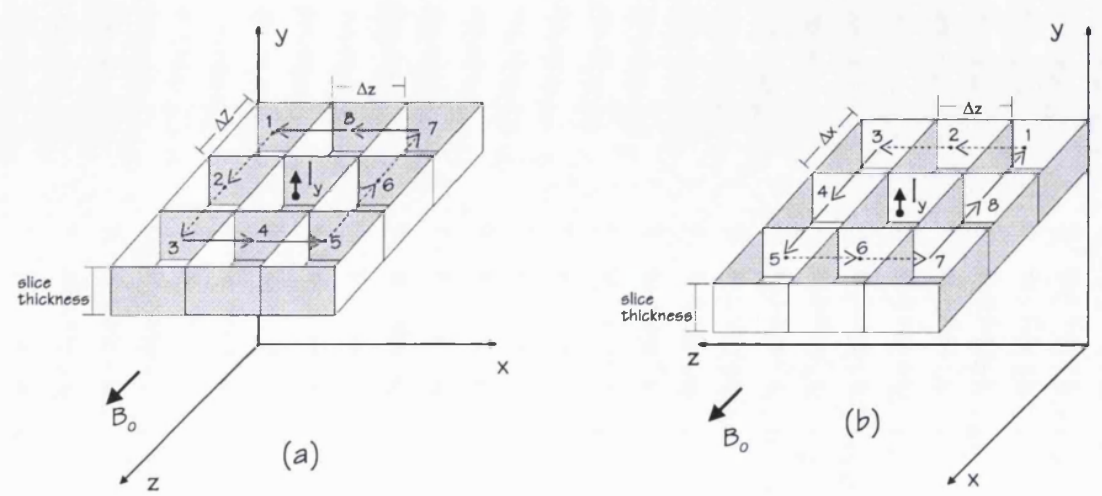

Figure 3.12 - The closed path used to compute the $I_{y}$ component of current.

Each simple integral is determined by the numerical trapezoidal rule for integration,

$$
\begin{aligned}
& \int_{a}^{b} f(x) d x=\frac{h}{2}\left[f\left(x_{1}\right)+2 f\left(x_{2}\right)+\ldots+2 f\left(x_{n-1}\right) f\left(x_{n}\right)\right] \\
& h=\frac{b-a}{n} \quad a=x_{1} \quad b=x_{n}
\end{aligned}
$$

where each path is divided into two parts, $n=2$. When the $z$-axis is parallel to $\boldsymbol{B}_{0}$, figure 3.12(a), the components $H_{35}$ and $H_{71}$ are equal to zero, and the integrals from 1 to 3 and 5 to 7 are equal to,

$$
\begin{aligned}
& \int_{1}^{3} H_{13} d L_{13}=\frac{2}{2} \frac{\Delta_{z}}{2}\left(H_{1}+2 H_{2}+H_{3}\right) \\
& \int_{5}^{7} H_{57} d L_{57}=-\frac{2}{2} \frac{\Delta_{Z}}{2}\left(H_{5}+2 H_{6}+H_{7}\right)
\end{aligned}
$$

In figure 3.12(b), $x$-axis parallel to $\boldsymbol{B}_{\theta}$, it is the current magnetic field in the $x$ direction that is measured. The integrals from 3 to 5 and 7 to 1 are then equal to,

$$
\begin{aligned}
& \int_{3}^{5} H_{35} d L_{35}=\frac{2}{2} \frac{\Delta_{x}}{2}\left(H_{3}+2 H_{4}+H_{5}\right) \\
& \int_{7}^{1} H_{71} d L_{71}=-\frac{2}{2} \frac{\Delta_{x}}{2}\left(H_{7}+2 H_{8}+H_{1}\right)
\end{aligned}
$$

The closed integral, equation (3.28), is then equal to, 


$$
\begin{aligned}
I_{y}=\oint \boldsymbol{H} \cdot d \boldsymbol{L} & =\frac{\Delta_{z}}{2}\left[\left(H_{1}+2 H_{2}+H_{3}\right)+\left(-H_{5}-2 H_{6}-H_{7}\right)\right] \\
& +\frac{\Delta_{x}}{2}\left[\left(H_{3}+2 H_{4}+H_{5}\right)+\left(-H_{7}-2 H_{8}-H_{1}\right)\right]
\end{aligned}
$$

\section{$I_{x}$ Current Calculation}

Consider now the component of current in the $x$-direction. This requires a set of three images to compose a closed path parallel to the $y z$-plane in order to apply Ampere's Law, as illustrated in figure 3.13.

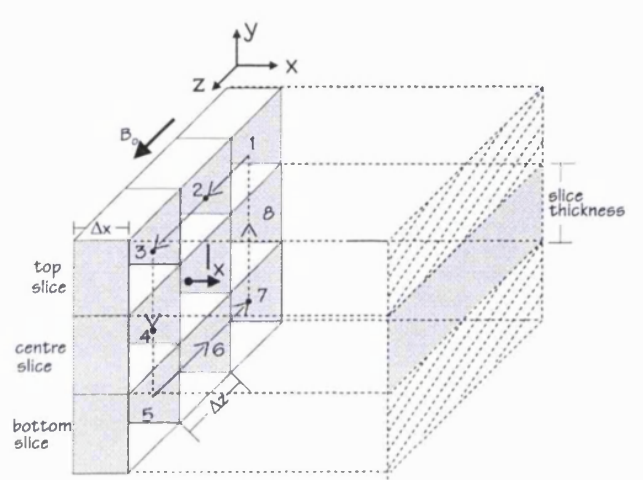

(a)

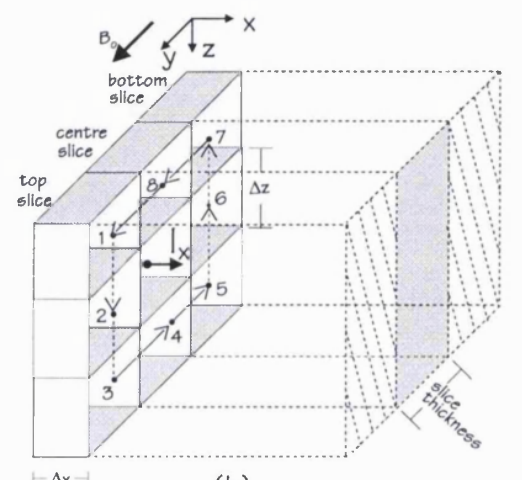

(b)

Figure 3.13 - The closed path used to calculate the $I_{x}$ component of current.

Similarly,

$$
I_{x}=\oint \boldsymbol{H} \cdot d \boldsymbol{L}=\int_{1}^{3} \boldsymbol{H}_{\mathbf{1 3}} d \boldsymbol{L}_{13}+\int_{3}^{5} \boldsymbol{H}_{35} d \boldsymbol{L}_{35}+\int_{5}^{7} \boldsymbol{H}_{57} d \boldsymbol{L}_{57}+\int_{7}^{1} \boldsymbol{H}_{71} d \boldsymbol{L}_{71}
$$

Now, with the $z$-axis parallel to $\boldsymbol{B}_{0}$, figure $3.13($ a), the integrals are equal to,

$$
\begin{aligned}
& \int_{1}^{3} \boldsymbol{H}_{13} d \boldsymbol{L}_{13}=\frac{2}{2} \frac{\Delta_{y}}{2}\left(H_{1}+2 H_{2}+H_{3}\right) \\
& \int_{5}^{7} \boldsymbol{H}_{57} d \boldsymbol{L}_{57}=-\frac{2}{2} \frac{\Delta_{y}}{2}\left(H_{5}+2 H_{6}+H_{7}\right)
\end{aligned}
$$

and with the $y$-axis parallel to $\boldsymbol{B}_{0}$, figure $3.13(\mathrm{~b})$, equal to,

$$
\begin{aligned}
& \int_{3}^{5} \boldsymbol{H}_{35} d \boldsymbol{L}_{35}=-\frac{2}{2} \frac{\Delta_{y}}{2}\left(H_{3}+2 H_{4}+H_{5}\right) \\
& \int_{7}^{1} \boldsymbol{H}_{71} d \boldsymbol{L}_{71}=\frac{2}{2} \frac{\Delta_{y}}{2}\left(H_{7}+2 H_{8}+H_{1}\right)
\end{aligned}
$$


The closed integral, equation (3.33), is then equal to,

$$
\begin{aligned}
I_{x}=\oint \boldsymbol{H} \cdot d \boldsymbol{L} & =\frac{\Delta_{z}}{2}\left[\left(H_{1}+2 H_{2}+H_{3}\right)+\left(-H_{5}-2 H_{6}-H_{7}\right)\right] \\
& +\frac{\Delta_{y}}{2}\left[\left(-H_{3}-2 H_{4}-H_{5}\right)+\left(H_{7}+2 H_{8}+H_{1}\right)\right]
\end{aligned}
$$

\section{Current Calculation}

Consider now the component of current in the $z$-direction. This also requires a set of images with three slices, as illustrated in figure 3.14.

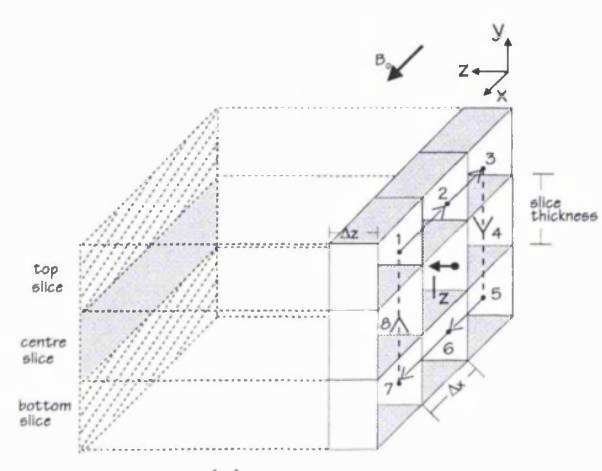

(a)

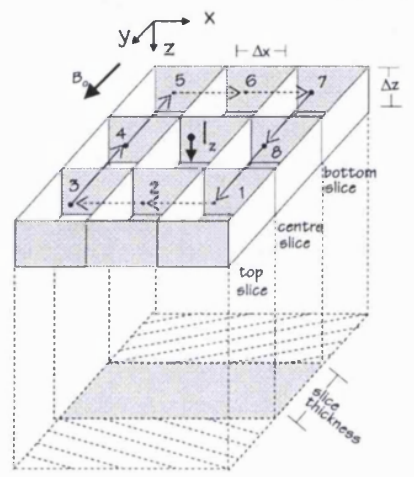

(b)

Figure 3.14 - The closed path used to compute the $I_{z}$ current component.

Similarly,

$$
I_{z}=\oint \boldsymbol{H} \cdot d \boldsymbol{L}=\int_{1}^{3} H_{13} d L_{13}+\int_{3}^{5} H_{35} d L_{35}+\int_{5}^{7} H_{57} d L_{57}+\int_{7}^{1} H_{71} d L_{71}
$$

In figure 3.14(a), $x$-axis parallel to $\boldsymbol{B}_{0}$, the integrals from 5 to 7 and from 1 to 3 are equal to,

$$
\begin{aligned}
& \int_{1}^{3} H_{13} d L_{13}=-\frac{2}{2} \frac{\Delta_{x}}{2}\left(H_{1}+2 H_{2}+H_{3}\right) \\
& \int_{5}^{7} H_{57} d L_{57}=\frac{2}{2} \frac{\Delta_{x}}{2}\left(H_{5}+2 H_{6}+H_{7}\right)
\end{aligned}
$$

and the integrals from 3 to 5 and from 7 to 1 are determined with the $y$-axis parallel to $\boldsymbol{B}_{\boldsymbol{0}}$, as shown in figure 3.14(b),

$$
\begin{aligned}
& \int_{3}^{5} H_{35} d L_{35}=-\frac{2}{2} \frac{\Delta_{y}}{2}\left(H_{3}+2 H_{4}+H_{5}\right) \\
& \int_{7}^{1} H_{71} d L_{71}=\frac{2}{2} \frac{\Delta_{y}}{2}\left(H_{7}+2 H_{8}+H_{1}\right)
\end{aligned}
$$


The integral in equation (3.37) is then equal to,

$$
\begin{aligned}
I_{z}=\oint \boldsymbol{H} \cdot d \boldsymbol{L} & =\frac{\Delta_{x}}{2}\left[\left(H_{5}+2 H_{6}+H_{7}\right)+\left(-H_{1}-2 H_{2}-H_{3}\right)\right] \\
& +\frac{\Delta_{y}}{2}\left[\left(-H_{3}-2 H_{4}-H_{5}\right)+\left(H_{7}+2 H_{8}+H_{1}\right)\right]
\end{aligned}
$$

\section{The template form of the current equations}

To apply equations (3.32), (3.36) and (3.40), they need to be written in a template form, composing a mask to be convolved $(\otimes)$ with the NMR phase images. As shown in figure 3.15 , the closed integral area is equal to $4 \Delta^{2}$, and the pixel area is equal to $\Delta^{2}$. Thus the area of integration needs to normalised, i.e., the closed integral result has to be divided by four.

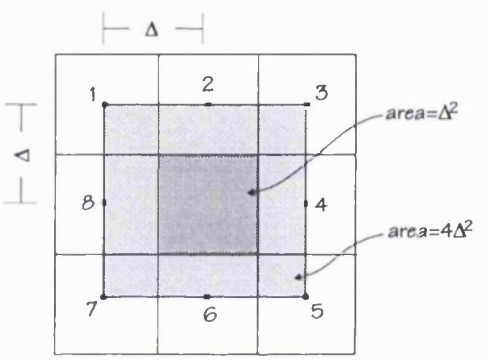

Figure 3.15 - Area normalisation to make the templates to convolute with the NMR phase images.

The template forms of equations (3.32), (3.36) and (3.40) are then equal to,

$$
\begin{gathered}
I_{y}=\frac{\Delta_{z}}{8}\left[\begin{array}{rrr}
1 & 0 & -1 \\
2 & 0 & -2 \\
1 & 0 & -1
\end{array}\right] \otimes H_{z}+\frac{\Delta_{x}}{8}\left[\begin{array}{rrr}
-1 & -2 & -1 \\
0 & 0 & 0 \\
1 & 2 & 1
\end{array}\right] \otimes H_{x} \\
I_{x}=\frac{\Delta_{z}}{8}\left[\begin{array}{rrr}
1 & 2 & 1 \\
0 & 0 & 0 \\
-1 & -2 & -1
\end{array}\right] \otimes\left[\begin{array}{c}
\text { top } \\
\text { centre } \\
\text { bottom }
\end{array}\right]_{B_{d} / / z}+\frac{\Delta_{y}}{8}\left[\begin{array}{lll}
-1 & 0 & 1 \\
-2 & 0 & 2 \\
-1 & 0 & 1
\end{array}\right] \otimes\left[\begin{array}{c}
\text { top } \\
\text { centre } \\
\text { bottom }
\end{array}\right]_{B_{d} / y} \\
I_{z}=\frac{\Delta_{x}}{8}\left[\begin{array}{rrr}
-1 & -2 & -1 \\
0 & 0 & 0 \\
1 & 2 & 1
\end{array}\right] \otimes\left[\begin{array}{c}
\text { top } \\
\text { centre } \\
\text { bottom }
\end{array}\right]_{B_{d} / / x}+\frac{\Delta_{y}}{8}\left[\begin{array}{ccc}
-1 & 0 & 1 \\
-2 & 0 & 2 \\
-1 & 0 & 1
\end{array}\right] \otimes\left[\begin{array}{c}
\text { top } \\
\text { centre } \\
\text { bottom }
\end{array}\right]_{B_{d} / / y}
\end{gathered}
$$

A program was implemented in " $\mathrm{C}$ " to apply the above templates and to compute 
the three orthogonal current components and the current magnitude. The routine needs to be fed with the current-on current-off difference images and also with the mask images created by the unwrapping program. The mask images are used to limit the process of current calculation to the object region in the FOV and to avoid the border of the images. If both sequences of current (positive-negative and negative-positive) are considered, a total of 27 images are read. The programme then applies equation (3.27) to determine the current magnetic field and the routines to convolve the templates with the field images. It also performs a surface integral to determine the total current in the image.

\section{Current Image Distribution and Maxwell's Equation}

Instead of computing the electrical current that passes through each pixel by applying Ampere's Law, it is also possible to compute the current density $(J)$ directly from Maxwell's equation.

$$
J=\nabla \times \boldsymbol{H}=\left(\frac{\partial H_{z}}{\partial y}-\frac{\partial H_{y}}{\partial z}\right) \hat{\boldsymbol{a}}_{x}+\left(\frac{\partial H_{x}}{\partial z}-\frac{\partial H_{z}}{\partial x}\right) \hat{\boldsymbol{a}}_{y}+\left(\frac{\partial H_{y}}{\partial x}-\frac{\partial H_{x}}{\partial y}\right) \hat{\boldsymbol{a}}_{z}
$$

Each partial derivative is a derivative of the NMR phase data, $\theta_{x}(\omega)$, which is calculated by the equation,

$$
\theta_{x}(\omega)=\arctan \left(\frac{I(\omega)}{R(\omega)}\right)
$$

The partial derivatives of $\theta_{x}(\omega)$ are calculated by,

$$
\begin{aligned}
& \frac{d}{d x}(\arctan u)=\frac{1}{1+u^{2}} \frac{d u}{d x} \\
& \frac{d}{d x}\left(\frac{u}{v}\right)=\frac{v\left(\frac{d u}{d x}\right)-u\left(\frac{d v}{d x}\right)}{v^{2}}
\end{aligned}
$$

Resulting in,

$$
\frac{d \theta(\omega)}{d x}=\frac{1}{\frac{R(\omega)^{2}+I(\omega)^{2}}{R(\omega)^{2}}} \frac{R(\omega)\left(\frac{d I(\omega)}{d x}\right)-I(\omega)\left(\frac{d R(\omega)}{d x}\right)}{R(\omega)^{2}}
$$




$$
\frac{d \theta(\omega)}{d x}=\frac{R(\omega)\left(\frac{d I(\omega)}{d x}\right)-I(\omega)\left(\frac{d R(\omega)}{d x}\right)}{R(\omega)^{2}+I(\omega)^{2}}
$$

The gradients for each part of equation (3.44) can be computed from the real and imaginary components and scaled to compose the current magnetic field. This procedure eliminates the need for the unwrapping procedure.

\subsection{Image registration}

In the last section we showed that to measure the three orthogonal field components, the object needs to be rotated inside of the NMR magnet. To perform this rotation the object is removed from the magnet centre, rotated and placed back inside. The magnetic field is again shimmed, and the pulse gain, resonance and slice position re-adjusted. This procedure moves the image in the FOV, hence before the current calculation it is necessary to register the images.

The process of image registration involves rotation and translation. First we choose one of the images as a reference and bring all other images to this position. To determine how much the images need to be rotated and moved inside of the FOV to reach the reference position, an algorithm was implemented in $\mathrm{C}$ language. The algorithm is divided into three sub-routines: 1) Image subtraction and display, 2) Image translation, and 3) Image rotation;

\section{Image subtraction}

First, using the region function of the UNC display program called "xdispunc", a mask of each image to be registered is produced. The mask has the pixels that match the object inside of the FOV set to unity and the others set to zero. The routine implemented to register the images reads the mask images, subtracts them and then displays the difference.

By an user interactive process, the routine now asks if one of the images, needs to be moved or rotated, executes the selected function and displays the difference. This process is repeated until most of the image difference is equal to zero.

\section{Image translation}

To move the image inside of the FOV, sub-routines which shift the image to the right, left, up or down can be selected. For each direction there is one function that is repeatedly called for the required number of times. Each time the function is called a 
pixel line or column is moved. The routine implemented does not move half pixels to avoid image interpolation.

\section{Image Rotation}

To rotate the image, each pixel position is considered as a vector and to rotate the image in relation to its centre, the vector origin is chosen at the centre of the FOV. The vector rotation is done using the following equations,

$$
\left(\begin{array}{l}
x_{\text {old }} \\
y_{\text {old }}
\end{array}\right)=\left(\begin{array}{cc}
\sin (\theta) & \cos (\theta) \\
\cos (\theta) & -\sin (\theta)
\end{array}\right)\left(\begin{array}{c}
x_{\text {new }} \\
y_{\text {new }}
\end{array}\right)
$$

where, $x_{\text {old }}$ and $y_{\text {old }}$ are the pixel position before the rotation, $x_{\text {new }}$ and $y_{\text {new }}$ are the vector position after the rotation, and $\theta$ is the required angle to rotate the image. First a new image matrix is generated, each vector in this new matrix is rotated back to original image using the above equations. If the rotated vector coordinates exceeds the original matrix size, the pixel value in the new matrix is set to zero. Also if the rotated vector coordinates are not integer values, they will not match exactly the coordinates in the original image, thus a two-dimensional interpolation function is called to determine the intermediate value in the original image. To perform the two-dimensional interpolation a Bicubic Spline function from Numerical Recipes in $\mathrm{C}$ is used. In each interpolation 5 pixels around the point to be interpolated are selected to fit the equation.

Figure 3.16 illustrates the images displayed by the program during the image registration process. These images are from the in vivo piglet experiment described in Chapter 8. Figure 3.16(a) shows the mask images and the differences before the image registration and figure 3.16(b) shows the mask images and the differences after being registered. Because during the NMR experiment the head is rotated in relation to the body it is not possible to register the neck region of the image.

Once the mask images are registered the number of pixels moved in each direction and the total angle rotated are printed. These values are then set in a Unix script file together with the command lines of the programs to move and rotate all the dispersion and absorption mode images acquired during the NMR experiment.

\subsection{Conclusion}

In this chapter the existing techniques to measure electrical current distribution 

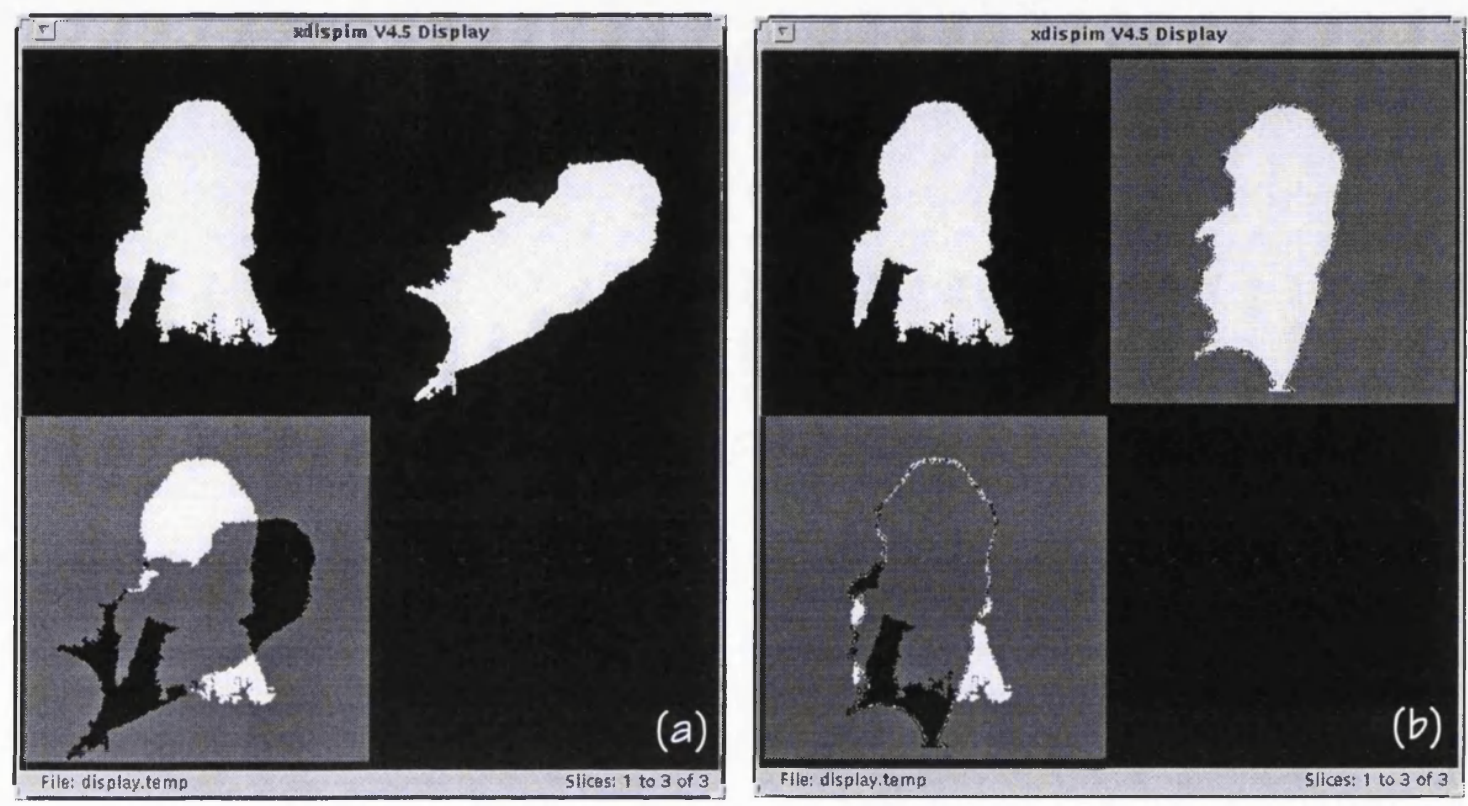

Figure 3.16 - Image displayed during the image registration. (a)before the image registration (b) after the image registration.

by NMR were reviewed and the technique chosen for the studies reported in this thesis described in detail.

The algorithm implemented to unwrap the phase images performed well. The major problem was concerning the interpolation routine, particularly when applied to unwrap the phase images acquired with an electrical current applied. These images do not have a linear phase change across the object, and the local linear interpolation algorithm implemented generally sets a low phase value to the pixels detected by the noise routines. Because of the relatively high noise in the data, polynomial interpolation also does not always give a good result. Therefore, most of these pixels were corrected manually and the values set were based on the values in the neighbourhood of the flagged pixels.

Considering the registration technique, we have also tried some automated techniques based on the image centre of mass and stochastic signal changes (Venot et al., 1984), but they did not work very well for the NMR images, even when applied to the phantom images which in general have a good SNR. Therefore we decided to implement the routine described above and make the registration manually. 


\section{Chapter 4}

\section{Simulation of the experimental phantom results}

\section{Current Magnetic Field Simulation}

This section describes a simulation performed to formally validate the experimental measurements of the electrical current distribution described in chapters 7 and 8 . The simulation was based on a cylindrical phantom constructed to perform the experiments. Many different aspects of the technique used to measure the electrical current using NMR were studied with this simulation.

The section starts by describing the phantom and the equations used to determine the current magnetic field. Following this, the field and current images are presented and a preliminary analysis of the first simulation results is done followed by a more accurate numerical simulation. During the simulation another aspect about the position of the wires that connect the current supply to the object been imaged was raised and it is analysed in the last section before the discussion and conclusion.

\subsection{Phantom description}

To perform the simulation and compare the theoretical results with those of the NMR experiment, a cylindrical shaped phantom was constructed. The advantage of choosing this shape is the simple analytical solution that it provides. The main disadvantages are that its current distribution has just one component along the cylinder axis, and also it has a uniform current density which gives a simple uniform current image. These problems are however less important than the advantage of being able to simulate the electric current induced magnetic field with the cylinder in a noise free manner.

The cylindrical phantom constructed is illustrated in figure 4.1. It consists of two concentric tubes made of perspex with inner diameters of $26.1 \mathrm{~mm}$ and $79 \mathrm{~mm}$, length $82 \mathrm{~mm}$ and wall thickness of $2 \mathrm{~mm}$ and $5 \mathrm{~mm}$ respectively. The cylinder bases are two $95 \times 95 \mathrm{~mm}$ squares of perspex $10 \mathrm{~mm}$ thick. In each base a circular groove was machined for each cylinder, with their centres matching the centre of the square bases. 


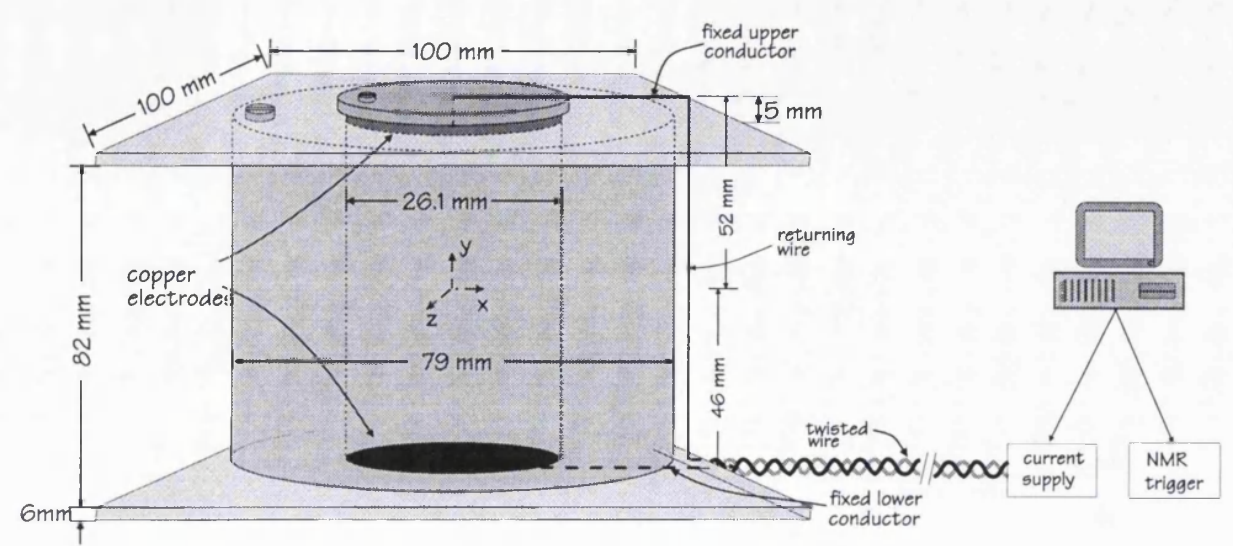

Figure 4.I - Cylindrical phantom used to compare the NMR experimental results with the field simulation.

Two $25 \mathrm{~mm}$ diameter electrodes made of copper were place in the top and bottom of the inner cylinder, and $1 \mathrm{~mm}$ holes in the top and bottom base centres enabled copper conductors to connect the electrodes to the current supply. In the bottom of the square base the copper conductor lies in a $2 \times 2 \mathrm{~mm}$ machined slot. Two nylon-screws holes in the upper square base make it possible to fill the cylinders.

The inner and outer cylinders were filled with a $5 \mathrm{mM}\left(\mathrm{T}_{1}\right.$ and $\left.\mathrm{T}_{2}=230 \mathrm{~ms}\right)$ and $10 \mathrm{mM}\left(\mathrm{T}_{1}\right.$ and $\left.\mathrm{T}_{2}=120 \mathrm{~ms}\right)$ copper sulphate $\left(\mathrm{CuSO}_{4}\right)$ solution respectively. The inner cylinder solution resistivity was measured using the symmetrical current pulses with a duty cycle of $20 \%$ for three different currents $(12.2 \mathrm{~mA}, 24.4 \mathrm{~mA}$ and $36.6 \mathrm{~mA})$. The resistivity average value was equal to $789 \Omega \mathrm{cm}$.

\subsection{The magnetic field equations}

The problem of determining the current magnetic field for the cylindrical phantom described above was divided into three parts: the magnetic field due to the current in the cylinder, that arising from the top and bottom wires and the magnetic field due to the returning wire on the side of the cylinder.

\section{- Cylinder magnetic field}

The magnetic field equations inside and outside of the inner cylinder are based on the diagram shown in figure 4.2, where $I$ is the electrical current applied through the electrodes in the $y$-direction, $l$ is the cylinder length and $r$ is the radius of the inner cylinder.

From the Biot-Savart law the magnetic field intensity at a point $P\left(x_{p}, y_{p}, z_{p}\right)$ produced by the current $I$ passing through the differential element $d \boldsymbol{L}$ is equal to (Hayt, 


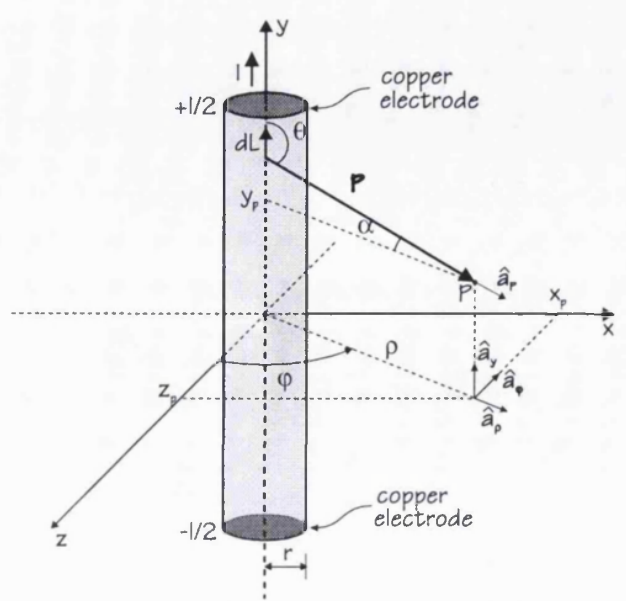

Figure 4.2- Axes reference used to determine the magnetic field arising from the electric current propagating in the inner cylinder.

1989),

$$
d \boldsymbol{H}_{P}=\frac{I}{4 \pi|\boldsymbol{P}|^{2}} d \boldsymbol{L} \times \hat{\boldsymbol{a}}_{P}
$$

where $d \boldsymbol{L}$ is the differential length in the current direction, equal to $d y \hat{\boldsymbol{a}}_{\boldsymbol{y}}$ and $\hat{\boldsymbol{a}}_{\boldsymbol{p}}$ is the vector unit in the $\boldsymbol{P}$ direction. The vector product is solved graphically using figure 4.2 ,

$$
d \boldsymbol{L} \times \hat{a}_{P}=d y \hat{a}_{y} \times \hat{a}_{P}=d y \sin \theta \hat{a}_{\varphi}
$$

where $\sin (\theta)=\sin (180-\theta)=\rho /|\boldsymbol{P}|$, and $|\boldsymbol{P}|=\sqrt{\rho^{2}+\left(y-y_{p}\right)^{2}}$. Applying equation (4.2) into (4.1) the total magnetic field $\boldsymbol{H}_{P_{o}}$ outside of the inner cylinder is given by the integral,

$$
\boldsymbol{H}_{P o}=\frac{I \rho}{4 \pi} \int_{-1 / 2}^{+l / 2} \frac{d y}{\left[\rho^{2}+\left(y-y_{p}\right)^{2}\right]^{3 / 2}} \hat{\boldsymbol{a}}_{\varphi}
$$

which is equal to ${ }^{12}$,

$$
\boldsymbol{H}_{P_{0}}=\frac{I}{4 \pi \rho}\left[\frac{\frac{l}{2}-y_{p}}{\sqrt{\left(\frac{l}{2}-y_{p}\right)^{2}+\rho^{2}}}-\frac{-\frac{l}{2}-y_{p}}{\sqrt{\left(-\frac{l}{2}-y_{p}\right)^{2}+\rho^{2}}}\right] \hat{\boldsymbol{a}}_{\varphi}
$$

12

$\int \frac{d u}{\left(u^{2}+a^{2}\right)^{3 / 2}}=\frac{u}{a^{2} \sqrt{u^{2}+a^{2}}}$ where $a^{2}=\rho^{2} \quad u=y-y_{p} \quad d u=d y$ 
If $P$ is inside of the inner cylinder and assuming a uniform current density, the induced magnetic field is determined by considering the amount of current enclosed in the area determined by the radius $\rho$ (Hayt, 1989),

$$
I_{e n c l}=I \frac{\rho^{2}}{r^{2}}
$$

applying (4.5) into (4.4) the magnetic field $\boldsymbol{H}_{P i}$ inside the inner cylinder is equal to,

$$
\boldsymbol{H}_{P i}=\frac{I \rho}{4 \pi r^{2}}\left[\frac{\frac{l}{2}-y_{p}}{\sqrt{\left(\frac{l}{2}-y_{p}\right)^{2}+\rho^{2}}}-\frac{-\frac{l}{2}-y_{p}}{\sqrt{\left(-\frac{l}{2}-y_{p}\right)^{2}+\rho^{2}}}\right] \hat{\boldsymbol{a}}_{\varphi}
$$

The rectangular components of equations (4.4) and (4.6) are equal to,

$$
\left(H_{P i}\right)_{r e t}=H_{P i} \frac{z}{\rho} \hat{a}_{x}-H_{P i} \frac{x}{\rho} \hat{a}_{z} \quad\left(H_{P o}\right)_{r e t}=H_{P o} \frac{z}{\rho} \hat{a}_{x}-H_{P o} \frac{x}{\rho} \hat{a}_{z}
$$

\section{- magnetic field from the top and bottom wires}

To determine the induced current magnetic field produced just by the top wire, consider the illustration in figure 4.3 where the wire goes from $x=0$ to $x=x_{w}$.

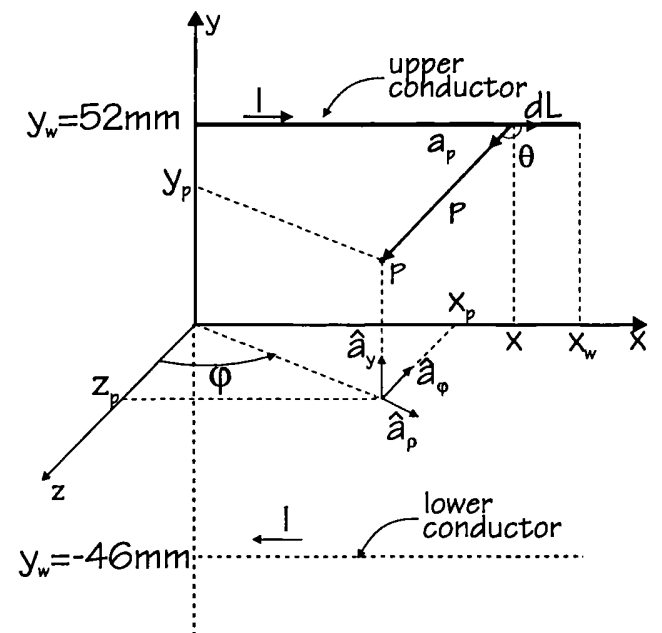

Figure 4.3 - Top wire position used determine the magnetic field produced by the electric current.

The magnetic field at a point $P\left(x_{p}, y_{p}, z_{p}\right)$ is given by equation (4.1) where $\boldsymbol{P}=\left(x_{p}-x\right) \hat{\boldsymbol{a}}_{\boldsymbol{x}}+\left(y_{p}-y_{w}\right) \hat{\boldsymbol{a}}_{\boldsymbol{y}}+z_{p} \hat{\boldsymbol{a}}_{z}$ and $d \boldsymbol{L}=d x \hat{\boldsymbol{a}}_{\boldsymbol{x}}$.

The vector product in equation (4.1) is equal to, 


$$
d \boldsymbol{L} \times \frac{\boldsymbol{P}}{|\boldsymbol{P}|}=\left|\begin{array}{ccc}
\hat{\boldsymbol{a}}_{\boldsymbol{x}} & \hat{\boldsymbol{a}}_{\boldsymbol{y}} & \hat{\boldsymbol{a}}_{z} \\
d x & 0 & 0 \\
\left(x_{p}-x\right) & \left(y_{p}-y_{w}\right) & z_{p}
\end{array}\right|=\frac{\left(y_{p}-y_{w}\right) d x \hat{\boldsymbol{a}}_{z}-d x z_{p} \hat{\boldsymbol{a}}_{\boldsymbol{y}}}{\sqrt{\left(x_{p}-x\right)^{2}+\left(y_{p}-y_{w}\right)^{2}+z_{p}^{2}}}
$$

Substituting equation (4.8) into (4.1), the total magnetic field at $P\left(x_{p}, y_{p}, z_{p}\right)$ due to the wire at the top of the cylinder $\left(\boldsymbol{H}_{P t}\right)$ is given by the integral,

$$
\boldsymbol{H}_{P t}=\frac{I}{4 \pi}\left[-z_{p} \hat{\boldsymbol{a}}_{y}+\left(y_{p}-y_{w}\right) \hat{\boldsymbol{a}}_{z}\right] \int_{x=0}^{x-x w} \frac{d x}{\left[\left(x_{p}-x\right)^{2}+\left(y_{p}-y_{w}\right)^{2}+z_{p}^{2}\right]}
$$

whose solution is equal to ${ }^{13}$,

$$
\boldsymbol{H}_{\boldsymbol{P t}}=\frac{I}{4 \pi}\left[-z_{p} \hat{\boldsymbol{a}}_{\boldsymbol{y}}+\left(y_{p}-y_{w}\right) \hat{\boldsymbol{a}}_{z}\right]\left|\frac{-\left(x_{p}-x\right)}{\left[\left(y_{p}-y_{w}\right)^{2}+z_{p}^{2}\right]\left[\left(x_{p}-x\right)^{2}+\left(y_{p}-y_{w}\right)^{2}+z_{p}^{2}\right]}\right|_{x=0}^{x-x_{w}}
$$

The magnetic field due to the wire in the bottom of the cylinder is given by the same equation (4.10) just making $I=-I$ and $y_{w}=-y_{w}$,

$$
\boldsymbol{H}_{P b}=\frac{-I}{4 \pi}\left[-z_{p} \hat{\boldsymbol{a}}_{y}+\left(y_{p}-y_{w}\right) \hat{\boldsymbol{a}}_{z}\right]\left|\frac{-\left(x_{p}-x\right)}{\left[\left(y_{p}-y_{w}\right)^{2}+z_{p}^{2}\right]\left[\left(x_{p}-x\right)^{2}+\left(y_{p}-y_{w}\right)^{2}+z_{p}^{2}\right]}\right|_{x=0}^{x-x_{w}}
$$

\section{- magnetic field due to the returning conductor}

Figure 4.4 shows the returning wire diagram used to determine the magnetic field generated by this part of the circuit.

Again, the magnetic field at $P\left(x_{p}, y_{p}, z_{p}\right)$ is given by equation (4.1) where $\boldsymbol{P}=\left(x_{P}-x_{w}\right) \hat{\boldsymbol{a}}_{\boldsymbol{x}}+\left(y_{P}-y\right) \hat{\boldsymbol{a}}_{\boldsymbol{y}}+\left(z_{P}-z\right) \hat{\boldsymbol{a}}_{z}, d \boldsymbol{L}=-d y \hat{\boldsymbol{a}}_{\boldsymbol{y}}$ and the cross product is equal to,

$$
d \boldsymbol{L} \times \frac{\boldsymbol{P}}{|\boldsymbol{P}|}=\left|\begin{array}{ccc}
\hat{a}_{x} & \hat{a}_{y} & \hat{a}_{z} \\
0 & -d y & 0 \\
\left(x_{p}-x_{w}\right) & \left(y_{p}-y\right) & z_{p}
\end{array}\right|=\frac{-z_{p} d y \hat{\boldsymbol{a}}_{x}+\left(x_{p}-x_{w}\right) d y \hat{\boldsymbol{a}}_{z}}{\sqrt{\left(x_{p}-x_{w}\right)^{2}+\left(y_{p}-y\right)^{2}+z_{p}^{2}}}
$$

substituting this value into equation (4.1) and solving the integral, the magnetic field at $P$ due to the returning wire is equal to,

13

$\int \frac{d u}{\left(u^{2}+a^{2}\right)^{\frac{3}{2}}}=\frac{u}{a^{2} \sqrt{u^{2}+a^{2}}}$ where $u=x_{p}-x \therefore d x=-d u$ and $a^{2}=\left(y_{p}-y_{w}\right)^{2}+z_{p}^{2}$ 


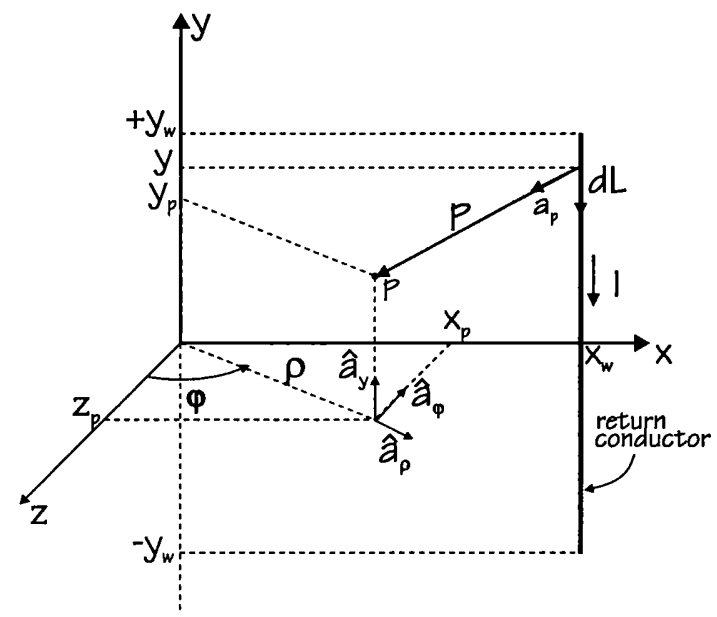

Figure 4.4 - Return wire position to determine the magnetic field produced by the electric current in it.

$$
\begin{aligned}
& \boldsymbol{H}_{P b}=\frac{I}{4 \pi}\left|\frac{-\left(y_{P}-y\right)}{a^{2}\left[\left(x_{p}-x_{w}\right)^{2}+\left(y_{p}-y\right)^{2}+z_{p}^{2}\right]^{1 / 2}}\right|_{y=-y_{w}}^{y-+y_{w}}\left[-z_{p} \hat{\boldsymbol{a}}_{x}+\left(x_{p}-x_{w}\right) \hat{\boldsymbol{a}}_{z}\right] \\
& a^{2}=\left[\left(x_{p}-x_{w}\right)^{2}+z_{p}^{2}\right]
\end{aligned}
$$

\subsection{Simulation and experiment results}

A C program was implemented to solve equations (4.4), (4.6), (4.10), (4.11) and (4.13) for three adjacent tomographic planes at $y_{p}=-3 \mathrm{~mm}, y_{p}=0$ and at $y_{p}=+3 \mathrm{~mm}$ along the $x y$-plane with the dimensions given in figure 4.1. The results were summed to determine the total magnetic field in each direction. Also included in the results were the magnetic fields due to the small sections of wires along the cylinder axis on the top and bottom of the cylinder that connect the upper and lower fixed wires to the electrodes. This field was calculate using equation (4.13) by making $x_{w}=0$.

An NMR experiment was also performed with the cylinder to measure the actual magnetic field produced by the electrical current and to compute the current images. To make sure that the NMR tomographic plane was in the cylinder centre, four capillaries were place into $2.1 \mathrm{~mm}$ wide by $1.5 \mathrm{~mm}$ deep adjacent slots on opposite sides of the cylinder wall. The reason for using several capillaries is because sometimes, depending on the shim, the abrupt magnetic susceptibility changes due to the cylinder solution, perspex and capillary glass mean that the capillary image may be shifted from the NMR tomographic plane. The diameter of the capillaries is $2 \mathrm{~mm}$ and each had a different length to help with the image registration. Three adjacent slices of $3 \mathrm{~mm}$ thickness were 
acquired for each orientation of the cylinder in relation to the main magnetic field. For each image 256 absorption and 256 dispersion mode spin-echos were acquired each one sampled 512 times. The echo-time was set to $60 \mathrm{~ms}$, the repetition time to $600 \mathrm{~ms}$, the field of view to $13 \mathrm{~cm}$, the current time to $60 \mathrm{~ms}$, and the current pulse amplitude to 12.2mA.

Figure 4.5 (a) shows the top, (b) the centre and (c) the bottom NMR images after they were reconstructed and registered. This set correspond to the images acquired with a positive-negative current sequence and with the $x$-axis (figure 4.1 ) parallel to $\boldsymbol{B}_{0}$. The capillary plane matches the NMR tomographic plane since their images do not appear in figures $4.5(\mathrm{a})$ and (c).
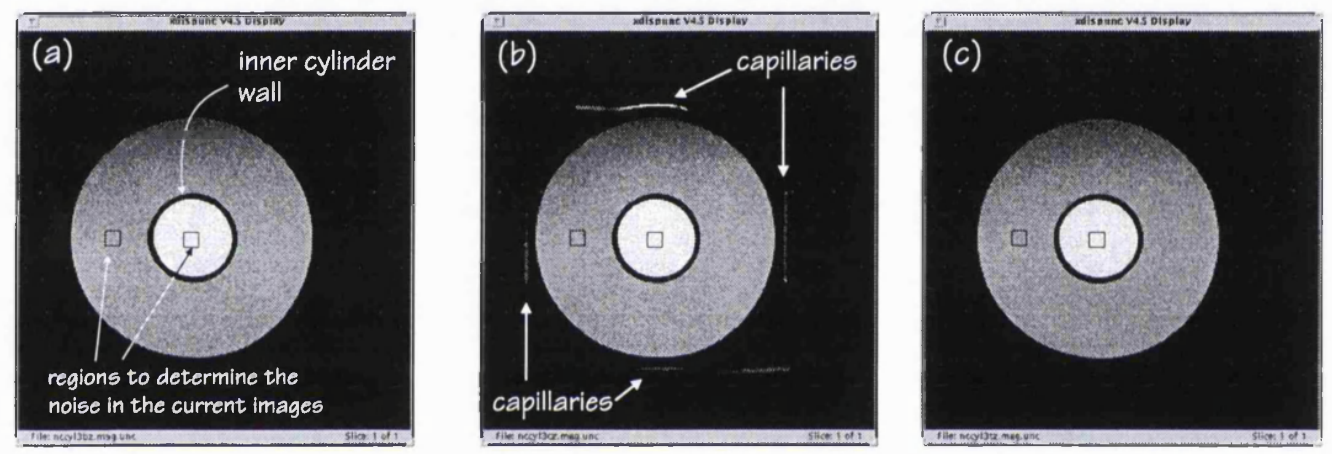

Figure 4.5 - NMR magnitude images to show the slice positions used to measure the current magnetic field. (a) $3 \mathrm{~mm}$ above (b) centre and (c) $3 \mathrm{~mm}$ down.

Figure 4.6 shows the current magnetic field components for the simulation and figure 4.7 the magnetic field measured from the NMR experiment after the phase images were reconstructed, registered, phase unwrapped and scaled by $1 /\left(\gamma T_{c}\right)$ to give the pixel values in Tesla. Only the field components at the centre slice position $\left(y_{p}=0\right)$ are shown. In figures 4.6 and 4.7 , (a), (b) and (c) are the electrical current magnetic field components in the $x, y$ and $z$ directions respectively, and (c) is the magnitude field component. 

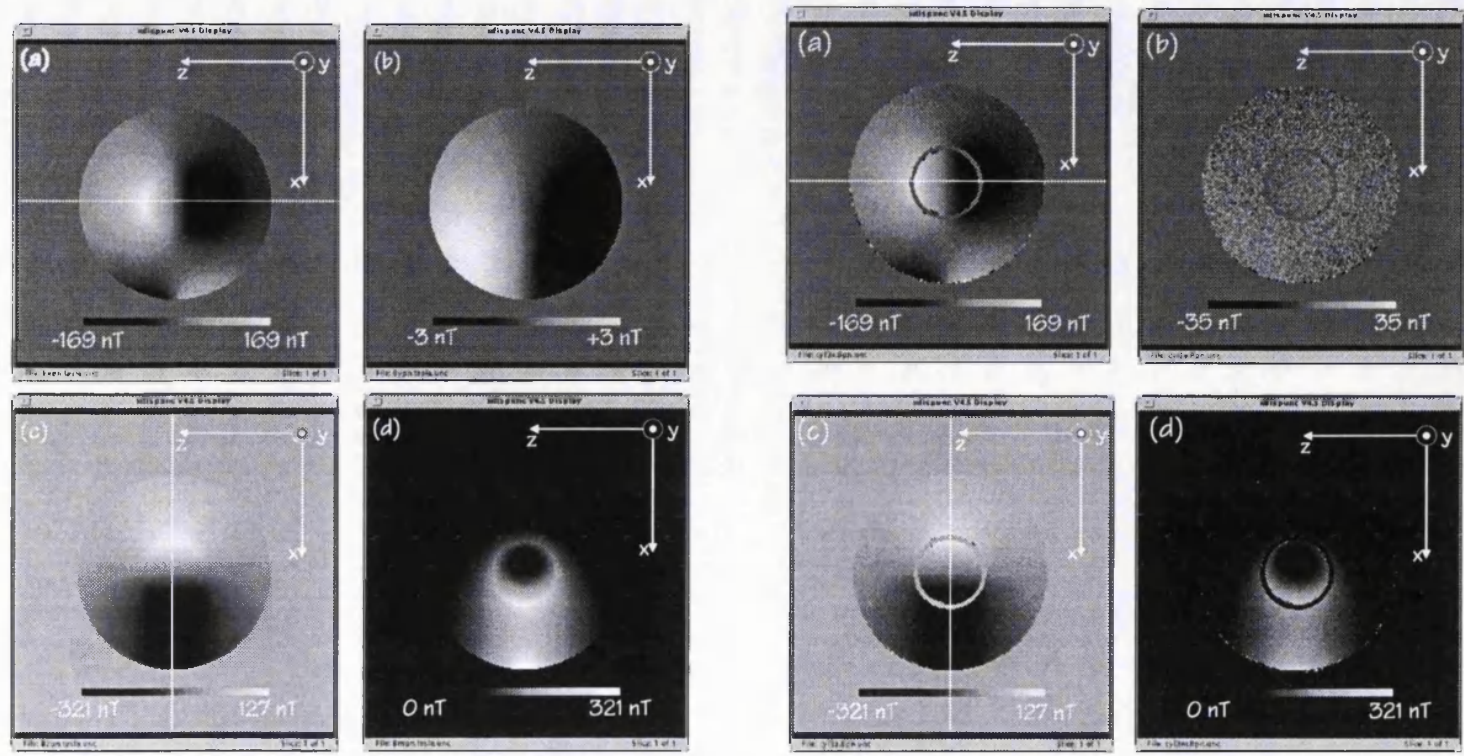

Figure 4.6 - Current magnetic field components from the simulation. (a) $x$-direction (b) $y$ direction (c) z-direction and (d) magnitude.

Figure 4.7- Current magnetic field components measured from the NMR experiment. (a) $x$ direction (b) y-direction (c) z-direction and $(d)$ magnitude.

Figure 4.8(a) shows a horizontal profile across the centre of figures 4.6(a) and 4.7 (a) and figure 4.8(b) a vertical profile in the centre of figures 4.6(c) and 4.7(d). The profiles show a very small difference between the inner field measured in the NMR experiment and that of the simulation. Also in figure $4.8(\mathrm{~b})$, the returning wire field produces a slightly bigger difference between the simulated and experimental field, probably due to the returning wire not being exactly at the centre of the side of the cylinder during the NMR experiment.

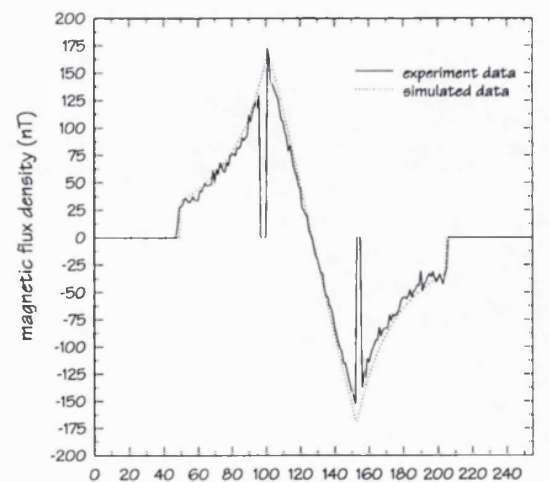

(a) horizontal position [pixel]

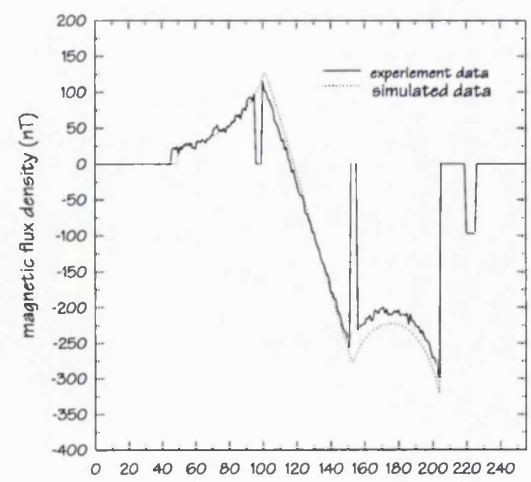

(b) vertical position [pixel]

Figure 4.8-One pixel wide profile of figures:(a)4.6a dotted line, 4.7 a full line; $(b) 4.6 c$ dotted line, $4.7 c$ full line 


\subsection{Electrical current images}

The electrical current components $I_{x}, I_{y}$ and $I_{z}$ were computed as described in section 3.4 for both the magnetic field measured by the NMR experiment and simulated with the $\mathrm{C}$ program. Figure 4.9 shows the $I_{y}$ component of the simulated field. Only the $I_{y}$ component is shown since the others are equal to zero. Figure 4.10 shows the $I_{x}, I_{y}$ and $I_{z}$ components of the NMR experiment. The components $I_{x}$ and $I_{z}$ are just the intrinsic NMR noise that propagates to the calculated current images.

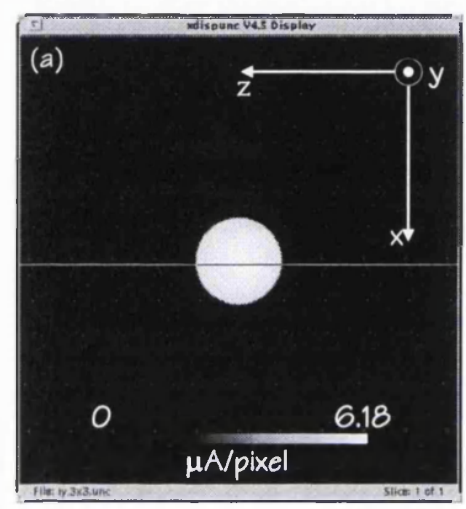

Figure 4.9 - Current image in the y-direction from the simulated magnetic field.
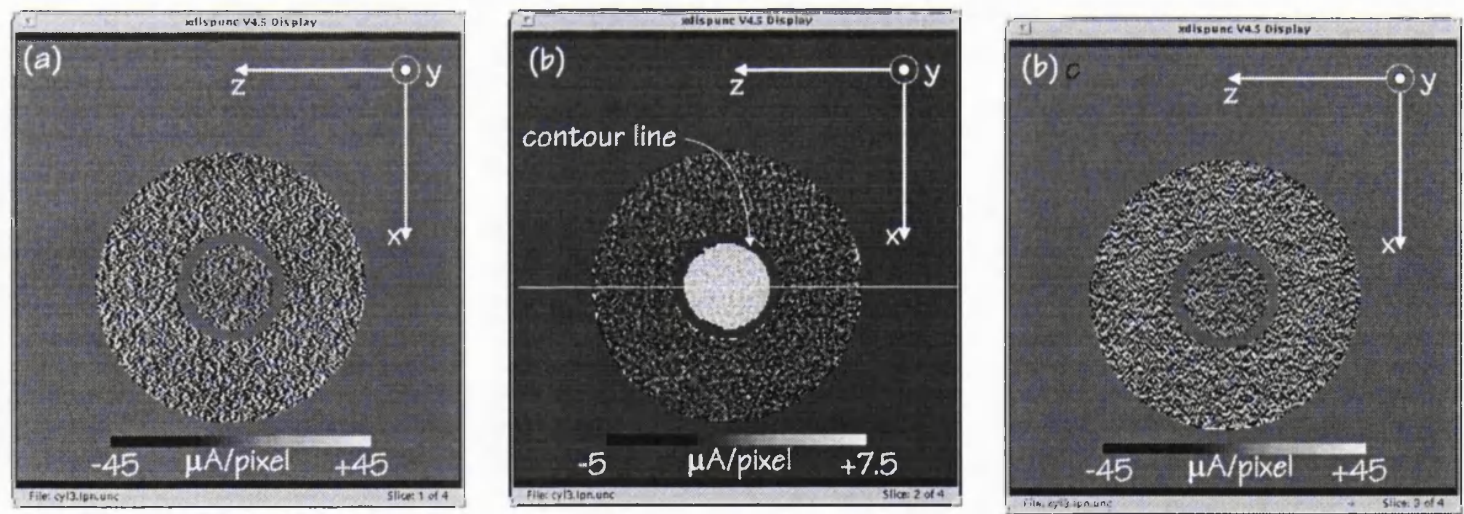

Figure 4.10-Current components from the NMR experiment. (a) $I_{x}$, (b) $I_{y}$ and (c) $I_{z^{*}}$

Figure 4.11 is a surface plot of the $I_{y}$ component to detail the image in figure 4.10(b). This surface plot was made by reducing the number of pixels from 256 by 256 to 36 by 36 using an averaging mask of 5 by 5 pixels and cutting off the image edges.

Figure 4.12 shows the horizontal image profile, one pixel wide, in the centre of the current images calculated from the simulated magnetic field (figure 4.9) and from the experimentally measured field (figure $4.10(\mathrm{~b})$ ). The profile from the simulated data 


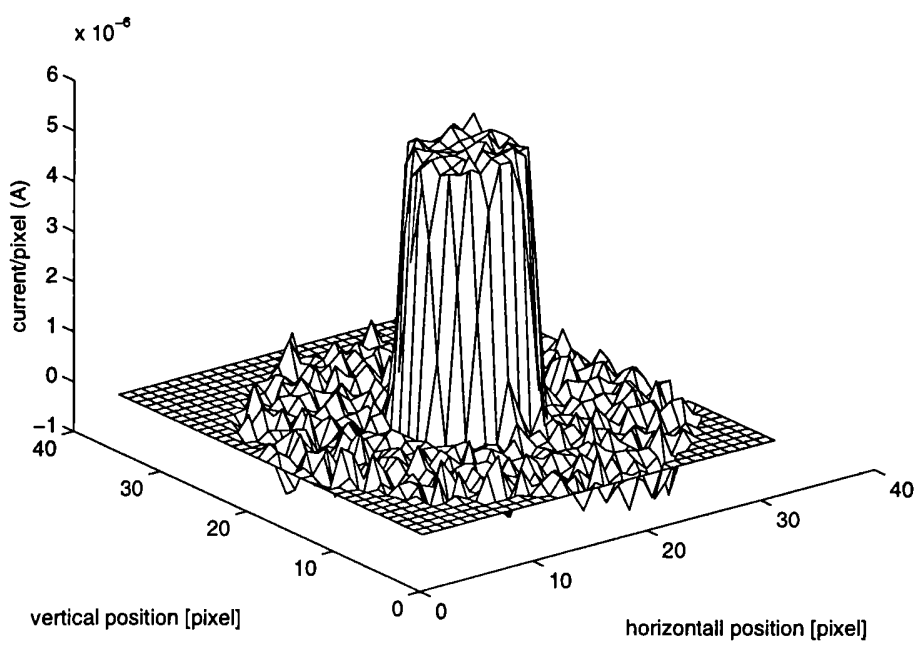

Figure 4.11 - Surface plot of figure 4.10(b).

shows a small non uniformity in the current distribution inside the inner cylinder. The reason for this were subsequently found to be due to the inappropriate use of equations applicable to wires of infinite length, since in this case, the cylinder length $(81 \mathrm{~mm})$ is comparable to its diameter $(26 \mathrm{~mm})$. A correct analysis is given in the next sub-section.

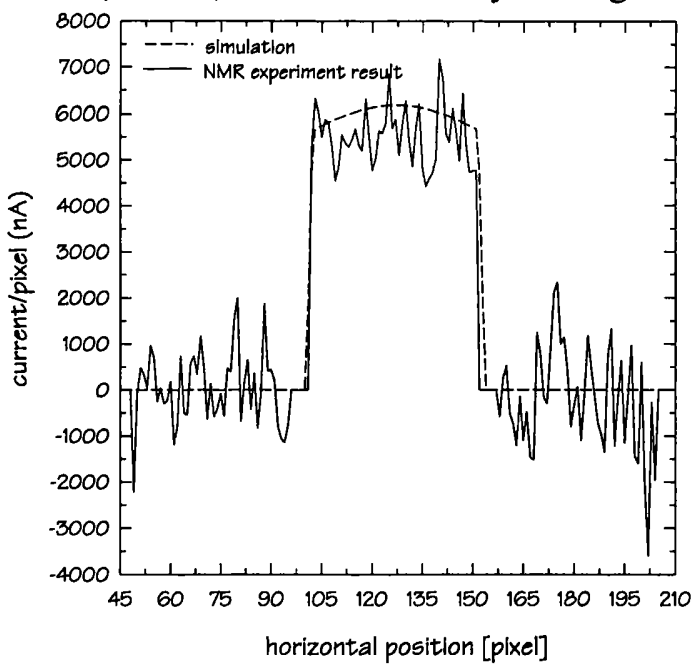

Figure 4.12 - Experimental and simulated horizontal centre profiles of the current images.

Calculations were performed to check if the total current applied was equal to the total current measured. For the simulated experiment the integrated current is equal to the total current applied $12.2 \mathrm{~mA}$ if the integrating region is equal to the outer cylinder diameter. If the integrating region is defined along the edge of the inner cylinder the total value is equal to $12.10 \mathrm{~mA}$. This difference is caused by the artifact produced by the closed integral mask (section 3.4) at the cylinders border between the regions with and without current. Because the integral mask and the cylinder field are symmetrical 
the artifact around the edge of the inner cylinder is cancelled when it is integrated. To view the artifacts the contrast in the current image shown in figure 4.9 was reduced to a range from $-3 \mathrm{nA}$ to $+3 \mathrm{nA}$, the stretched image is shown in figure 4.13.

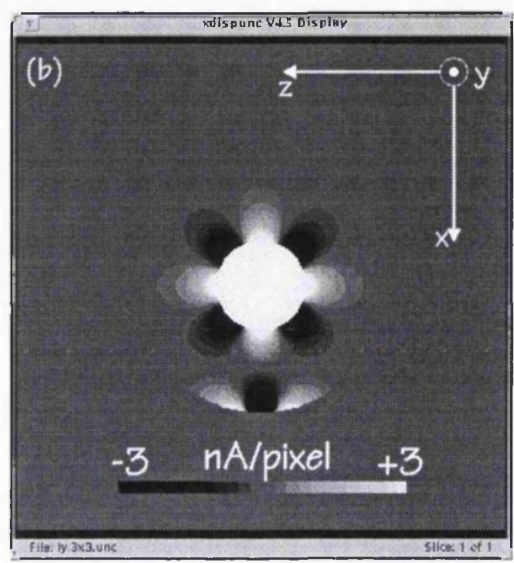

Figure 4.13 - Artifacts in the cylinder current image showed in figure 4.9.

For the NMR experiment, the current within the inner cylinder was integrated by defining the contour region shown in figure 4.10(b). This contour line was automatically defined by the display program xdispunc. The result is equal to $10.37 \mathrm{~mA}$. The difference is partially related to the area lost during the current calculation in order to avoid the border of the image. For the NMR experiment the inner cylinder wall was made of perspex which does not appear in the NMR images (figure 4.5) and the phase values are set to zero. During the current calculation whenever a pixel of the mask reaches the edge, the current is set to zero. That is the reason why the cylinder wall appears bigger in the current images (figure 4.10) compared to the NMR images (figure 4.5). The region area measured in the current image is equal to $502.08 \mathrm{~mm}^{2}$ (number of pixels in the region $=1947$, fov $=13 \mathrm{~cm}$ and image matrix 256 by 256). The cylinder area is equal to $535.02 \mathrm{~mm}^{2}$ (inner diameter $26.1 \mathrm{~mm}$ ). The area lost is equal to $32.94 \mathrm{~mm}^{2}$. If the current is considered to be uniform, the current density in the cylinder is equal to $22.80 \mu \mathrm{A} / \mathrm{mm}^{2}\left(12.2 \mathrm{~mA} / 535.02 \mathrm{~mm}^{2}\right)$ which means that the current missing is equal to $0.751 \mathrm{~mA}$. Adding this result to the integrated current result gives a value of $11.12 \mathrm{~mA}$. Therefore the integrating error to consider to be equal to $8.85 \%$.

Approximately the same error is found by comparing the current per pixel instead of integrated current. The mean current of a region of 5 by 5 pixels in the centre of figure 4.10 (b) is equal to $5.26 \mu \mathrm{A} /$ pixel or $20.42 \mu \mathrm{A} / \mathrm{mm}^{2}$. Comparing this value with the theoretical current density expected, $22.80 \mu \mathrm{A} / \mathrm{mm}^{2}$, the error is equal to $10.45 \%$. 
A noise analyses was also performed to quantify the standard deviation in the magnetic field image and in the current image (just for the $I_{y}$ component). The phase standard deviation, $\sigma(\varphi)$, in the NMR phase image, is determined on a pixel by pixel basis by the ratio $\sigma(|M|) /|M|$ (equation (6.21)), where $\sigma(|M|)$ is the signal standard deviation and $|M|$ is the signal intensity. This ratio was determined by placing two regions of $10 \times 10$ pixels one in the inner cylinder and another in the outer cylinder. Just one set of images (top, centre and bottom slices) with the cylinder $x$-axis parallel to $\boldsymbol{B}_{\boldsymbol{0}}$ was used. The regions are indicated in figure 4.5. For each region the standard deviation was calculated and the above ratio determined pixel by pixel. The average standard deviation of each region was then calculated. The expected current standard deviation that propagates from the noise in the NMR phase images to the current image is given by (section 6.3),

$$
\sigma\left(I_{y}\right)=\frac{\sqrt{2}}{4 \gamma T_{C} \mu_{0}} \frac{\sigma(|M|)}{|M|} \sqrt{3\left(\Delta x^{2}+\Delta z^{2}\right)}
$$

The results for the noise analysis are shown in table 4.1. The predicted average standard deviation in the current image for the inner cylinder region is equal to $0.70 \mu \mathrm{A}$ and in the outer cylinder region $1.08 \mu \mathrm{A}$ which are very near to the measured values using in the current images. For the field image the mean standard deviations are equal to $1.84 \mathrm{nT}$ and $2.88 \mathrm{nT}$, inside and outside the inner cylinder respectively. These explain why it is not possible to measure the magnetic field shown in figure $4.6(\mathrm{~b})$, where the range of image values is $\pm 3 \mathrm{nT}$.

Table 4.1 - Noise analyses results to quantify the standard deviation in the current image.

\begin{tabular}{|c|c|c|c|c|c|c|c|c|}
\hline \multirow{2}{*}{ region } & \multirow{2}{*}{$\begin{array}{l}\text { figure } \\
3.5\end{array}$} & \multicolumn{2}{|c|}{ magnitude } & \multicolumn{2}{|c|}{ phase (degree) } & \multirow{2}{*}{$\begin{array}{c}{ }^{* *} \sigma(B) \\
(n T)\end{array}$} & \multirow{2}{*}{$\begin{array}{c}{ }^{*} \sigma(I) \\
(\mu A)\end{array}$} & \multirow{2}{*}{$\begin{array}{r}{ }^{* *} \sigma(I) \\
(\mu A)\end{array}$} \\
\hline & & $\sigma(M)$ & $<M>$ & ${ }^{*}<\sigma(\varphi) / M>$ & $" \sigma(\varphi)$ & & & \\
\hline \multirow{3}{*}{ inner } & $a$ & 14.66 & 450.33 & 1.87 & 2.08 & 1.44 & \multirow{3}{*}{0.706} & 0.711 \\
\hline & $b$ & 14.40 & 451.85 & 1.83 & 2.04 & 2.27 & & 0.695 \\
\hline & $c$ & 14.39 & 450.16 & 1.83 & 2.21 & 2.40 & & 0.698 \\
\hline \multirow{3}{*}{ outer } & $a$ & 14.36 & 290.21 & 2.81 & 3.14 & 3.40 & \multirow{3}{*}{0.881} & 1.07 \\
\hline & $b$ & 14.73 & 290.76 & 2.91 & 3.36 & 3.67 & & 1.10 \\
\hline & $c$ & 14.37 & 294.02 & 2.83 & 3.32 & 3.62 & & 1.06 \\
\hline
\end{tabular}

* measured values ${ }^{* *}$ predicted values 


\subsection{Preliminary analyses of the results}

In figure 4.12 the dashed profile of the current calculated from the simulated field showed a slight non-uniformity across the inner cylinder. This non-uniformity should not exist since the electrodes completely fill the ends of the inner cylinder.

The magnetic field in the inner cylinder was determined using the same approach described in electromagnetism books for the field inside of conductors (Hayt, 1989; Ramo et al., 1993). The difference between the equations of the magnetic field inside and outside of the inner cylinder is that inside of the inner cylinder just the current enclosed in the area determined by the radius $\rho$ is included, as we did using equation (4.5). This procedure implies that to determine the magnetic field inside the inner cylinder, the total current applied was not considered to flow in one line as assumed when calculating the field outside of the inner cylinder, but rather a uniform current distribution across the cylinder was assumed. We also determined the magnetic field of a complete circuit, not just that of a finite element of current that started at $-l / 2$ and finished at $+l / 2$ (figure 4.2 ), which would be the case if just the cylinder field was used.

By looking at the field equations (4.4) and (4.6), if the cylinder length is made much bigger than its diameter the square brackets are approximately equal to two, and the resulting field is then given by the normal equation found in text books ( $I=H 2 \pi \rho)$. Therefore we concluded that it is not completely correct to apply Biot-Savart law in a simple way to our cylinder case because its dimensions, (length and diameter) are comparable.

The correct approach to determine the magnetic field produced by the inner cylinder would be by developing a general equation of the magnetic field at a point $P\left(x_{p}\right.$, $\left.y_{p}, z_{p}\right)$ produced by an infinitesimal line of current at any position $w\left(x_{w}, y_{w}, z_{w}\right)$. From this equation the applied current $I$ is replaced by the current density $J d x d z$, and the magnetic field at $P$, determined by the surface integral along $x$ - and $z$-. Basically this equation had already been developed for the magnetic field due to the returning wire, so we just have to replace in equation (4.13) $I$ by $-\delta$ and $z p$ by $(z p-z w)$,

$$
\begin{aligned}
& \left.\boldsymbol{H}_{\boldsymbol{P}}=\frac{J}{4 \pi} \int_{-x_{p}}^{+x_{p}} \int_{-z_{p}}^{+z_{p}}\left|\frac{-\left(y_{p}-y\right)}{a^{2}\left[\left(x_{p}-x_{w}\right)^{2}+\left(y_{p}-y\right)^{2}+\left(z_{p}-z_{w}\right)^{2}\right]^{1 / 2}}\right|_{y--y_{w}}^{y-+y_{w}}\left[\left(z_{p}-z_{w}\right) \hat{a}_{x}-\left(x_{p}-x_{w}\right) \hat{\boldsymbol{a}}_{z}\right] d x d z_{(4)}\right) \\
& a^{2}=\left[\left(x_{p}-x_{w}\right)^{2}+\left(z_{p}-z_{w}\right)^{2}\right]
\end{aligned}
$$


Because the surface integral in this equation does not have a simple analytical solution, a C program was implemented to numerically solve it. Basically the program divides the inner cylinder into $n(\mathrm{n}=2077)$ current pixels, each one representing $I / n$ of the total current applied. For each current pixel the magnetic field in the $x$ - and $z$ directions were determined for all remaining pixels within the outer cylinder which includes the inner cylinder pixels except for the selected current pixel where both components are set equal to zero. The sum of the field produced by each current pixel gives the total field at a position $P$.

The results of the numerical calculation were added to the magnetic field produced by the surrounding wires. The resulting magnetic field images in the $x$ - and $z$-directions are shown in figure 4.14(a) and (b) respectively. The $B_{y}$ component is the same as shown in figure $4.6(\mathrm{~b})$.
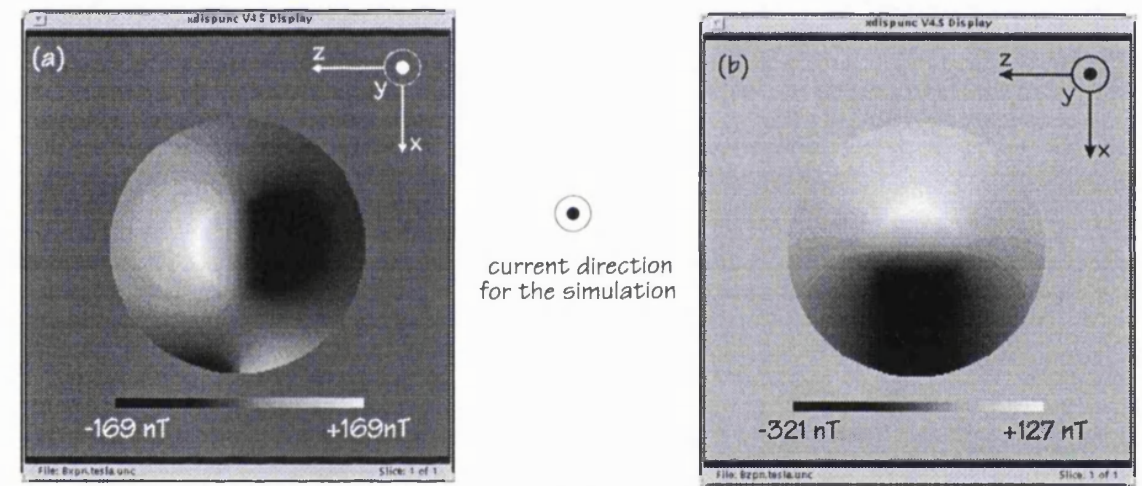

Figure 4.14- Numerical simulated magnetic field components of the cylinder using equation 3.15. (a) $x$ direction and (b) y-direction.

Using the same current program, the current image in the $y$-direction was computed. Figure 4.15(a) show the calculated image and figure 4.15(b) is the profile in the centre of it.

The simulated current image in figure 4.15 now has a flat current distribution which proves that no error exist in the process of calculating the current image from the magnetic field. The differences between the previous analytical solution and the now numerically calculated field components in the $x$ - and $z$-directions can be seen in figure $4.16(a)$ and (b).

The range of values in figure 4.16 shows that at most the difference between the analytical solution using equations (4.4) and (4.6) and that using the numerical solution of equation (4.15) is $\pm 3 \mathrm{nT}$, which is very small compared to the range of values of the 

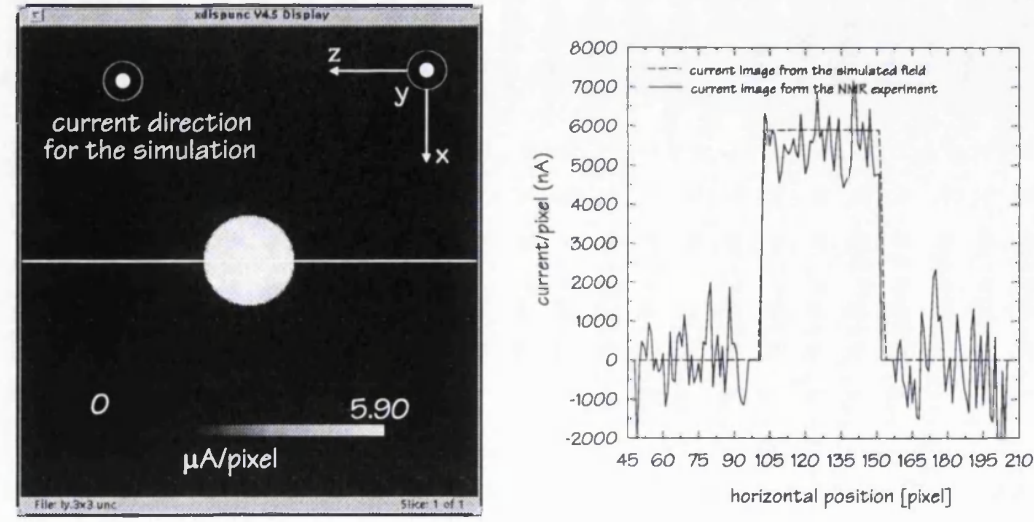

Figure 4.15-Electrical current image calculated from the simulated field component shown in figure 4.14 and the one pixel wide image profile.
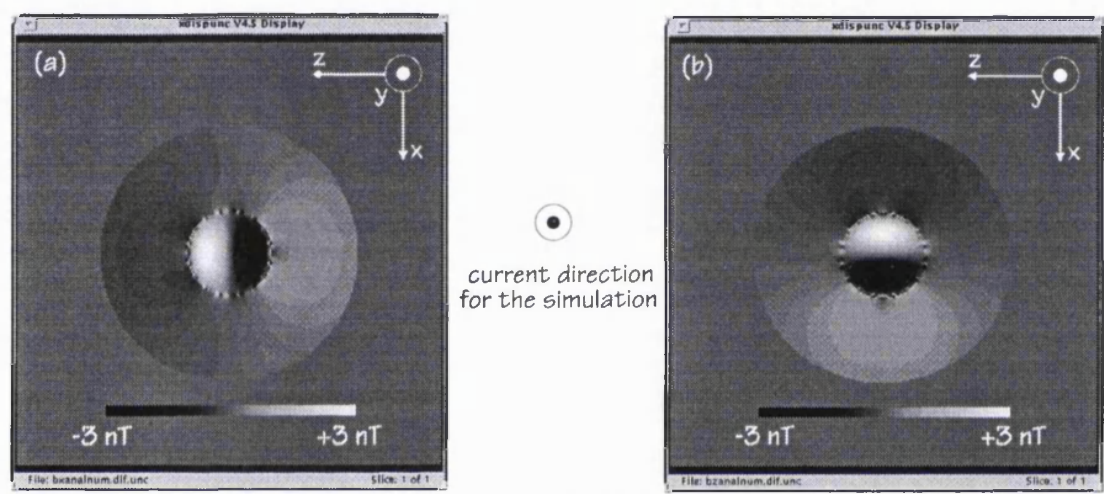

Figure 4.16- The difference between the simulated field shown in figure 4.6 and in figures 4.14. (a) the $x$ component and (b) z component.

field images in figures 4.6 and 4.14 .

To compare the numerically simulated field and the field measured in the NMR experiment, the image profiles are shown in figure 4.17(a) for the field component in the $z$-direction and 4.17(b) for the field component in the $x$-direction.

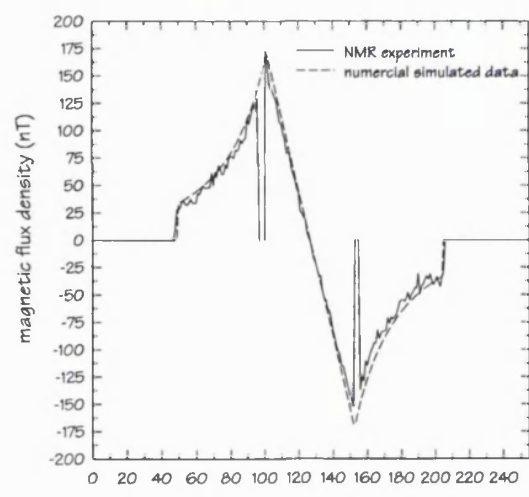

(a) horizontal position [pixel]

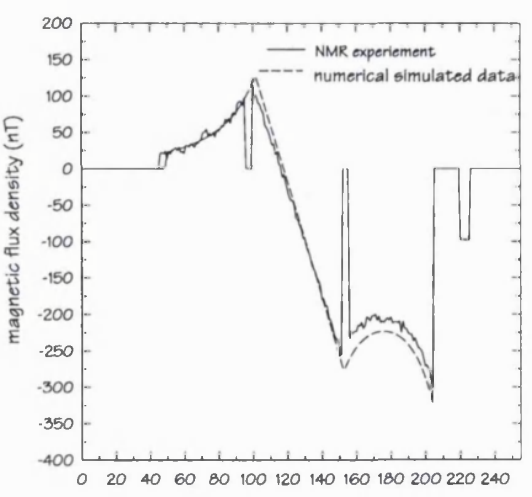

(b) vertical position [pixel]

Figure 4.17- Profiles of the numerical simulated field and that of the NMR experiment 


\subsection{The infinitely long wire case}

It is also necessary to consider the effects of the wire positions used for the in vitro experiments (Chapter 7) performed prior to the development of this simulation. In these experiments, the wires that connected to the power supply did not make a complete loop around the head as was done for the cylinder phantom simulation. During these experiments just the wire length going from the top of the head to the border of the frame (figure 7.1) was rotated with the head, the remaining wire being kept parallel to the main NMR field. Hence the returning wire field that would complete the magnetic circuit enabling us to apply Ampere's Law was not measured. This can be noticed in the magnetic field images of the in vitro piglet results (figures 7.3 and 7.15).

In theory it is not necessary to worry about the wire positions during the NMR experiment. Even though their magnetic field is measured, their current flows outside the imaged object and does not contribute to the $\oint \boldsymbol{H} \cdot d \boldsymbol{L}$ results. In practice however, keeping them as far away as possible may help to avoid unnecessary $2 \pi$ transitions in the NMR phase images. On the other hand, keeping the wires always parallel to the main magnetic field introduces another error in the current calculation. As explained here, this error for the cylindrical phantom is very small and may not affect at all the in vitro experiment results obtained with the piglet head where no symmetry exists and the current distribution is completely non uniform.

To look at this problem let us considered here that the wires used to connect the cylindrical phantom to the current source were always kept parallel to the main field and that just the field in the cylindrical phantom is measured in the NMR experiment. Since the wires are very long (because the current supply is placed far from the NMR magnet), they can be considered as having an infinite length. Therefore to determine their magnetic field contribution, the limit in equation (4.9) has to be change from $x=x_{w}$ to $x=\infty$,

$$
\boldsymbol{H}_{\boldsymbol{P t}}=\frac{I}{4 \pi}\left[-z_{p} \hat{\boldsymbol{a}}_{\boldsymbol{y}}+\left(y_{p}-y\right) \hat{\boldsymbol{a}}_{z}\right]\left|\frac{-\left(x_{p}-x\right)}{\left[\left(y_{p}-y\right)^{2}+z_{p}^{2}\right]\left[\left(x_{p}^{2}-x\right)^{2}+\left(y_{p}-y\right)^{2}+z_{p}^{2}\right]}\right|_{x=0}^{x=\infty}
$$

The infinite case is solved by applying L'Hospital's rule, 


$$
\begin{aligned}
& \lim _{x \rightarrow \infty} \frac{x-x_{p}}{k^{2}\left[\left(x_{p}-y\right)^{2}+k^{2}\right]^{1 / 2}} \quad \text { where } k^{2}=\left(y_{p}-y_{W}\right)^{2}+z_{p} \\
& \frac{\partial\left(x-x_{p}\right)}{\partial x}=1 \text { and } \frac{\partial}{\partial x}\left[\left(x_{p}-x\right)^{2}+k^{2}\right]^{1 / 2}=\frac{1-\frac{x_{p}}{x}}{\left[\left(\frac{x_{p}-1}{x}\right)^{2}+\frac{k^{2}}{x^{2}}\right]^{1 / 2}}
\end{aligned}
$$

$$
\lim _{x \rightarrow \infty} \frac{x-x_{p}}{k^{2}\left[\left(x_{p}-x\right)^{2}+k^{2}\right]^{1 / 2}}=\frac{1}{k^{2}}
$$

and the magnetic field at $P$ due to the infinitely long wires is equal to,

$$
\boldsymbol{H}_{p}=\frac{I}{4 \pi}\left[\frac{\left[x_{p}^{2}+\left(y_{p}-y_{w}\right)^{2}+z_{p}^{2}\right]^{1 / 2}+x_{p}}{\left[\left(y_{p}-y_{w}\right)^{2}+z_{p}^{2}\right]\left[x_{p}^{2}+\left(y_{p}-y_{w}\right)^{2}+z_{p}^{2}\right]^{1 / 2}}\right]\left[-z_{p} \hat{\boldsymbol{a}}_{y}+\left(y_{p}-y_{w}\right) \hat{\boldsymbol{a}}_{z}\right]
$$

Equation (4.18) shows that even for very long wires parallel to the main magnetic field aligned along the $x$-axis of the cylinder, the current magnetic field components are in the $y$ - and $z$-directions. Since the precession frequency of the nuclear spins is just affected by the magnetic field parallel to $\boldsymbol{B}_{\boldsymbol{0}}$, these components are not measured by the NMR experiment. The same will happen when the cylinder is rotated and its $z$-axis is parallel to $\boldsymbol{B}_{\boldsymbol{0}}$. Therefore we are left with just the field from a finite element conductor where the current starts at $y=-l / 2$ and finished at $y=+l / 2$ (figure 4.2) and the magnetic field is given by the numerical solution of equation (4.15).

To confirm this approach an NMR experiment was performed with the same cylindrical phantom shown in figure 4.1. The connecting wires were changed so they could always be kept parallel to the NMR field. For the experiment the field of view was set to $15 \mathrm{~cm}$, repetition time $1000 \mathrm{~ms}$, echo-time and current-time $60 \mathrm{~ms}$ and slice thickness $3 \mathrm{~mm}$. The slices were acquired in the cylinder centre with a current amplitude of $12.2 \mathrm{~mA}$. The same solution describe before was used to fill up the cylinders. Just two slices were acquired for this experiment, one with the $x$-axis parallel to $\boldsymbol{B}_{\boldsymbol{0}}$ and another with the $z$-axis. Therefore just the current magnetic field in the $y$-direction can be determined. Figures 4.18(a) and (c) shows the numerical solution of equation (4.15), no other field was added to this field, and figures 4.18(b) and (d) show the actual magnetic 
field components measured in the NMR experiment.
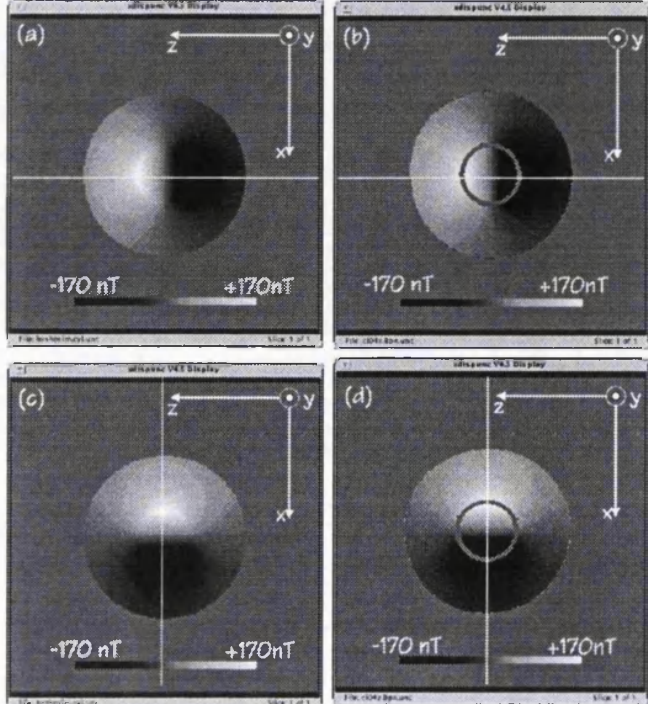

Figure 4.18-Simulated magnetic field $(a)$ in the $x$-direction $(c)$ in the $z$-direction. NMR field measurements (b) $x$-direction and (d) z-direction.

Figure 4.19(a) shows the horizontal profiles of figure 4.18(a) and (b) and figure 4.19(b) the vertical profiles of figure 4.18(c) and (d). The profiles show a reasonably good agreement between these field images.

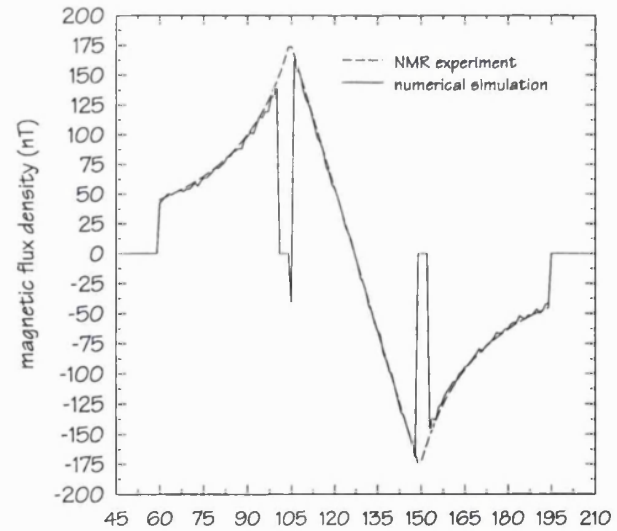

(a) horizontal position [pixel]

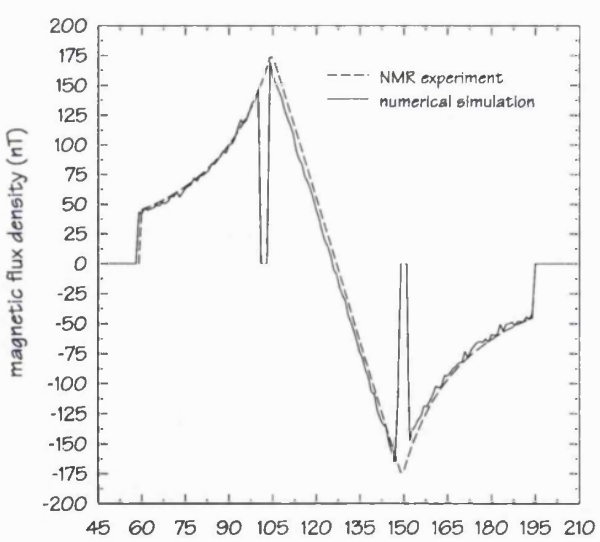

(b) vertical position [pixel]

Figure 4.19- Image profiles of a one pixel slice of figure 4.18, (a) from images (a) and (b), (b) from images $(c)$ and $(d)$.

In figure 4.20 is shown the current image surface plot obtained from the NMR experiment, and in figure 4.21 is shown the current image surface plot obtained from the numerical simulation. Just the induced magnetic field produced by the current 
propagating in the cylindrical phantom is considered in the current calculation and measured in the NMR experiment. These surface plots were made by reducing the number of pixels from $256 \times 256$ to $36 \times 36$ using an average mask of 5 by 5 pixels and cutting off the image edges.

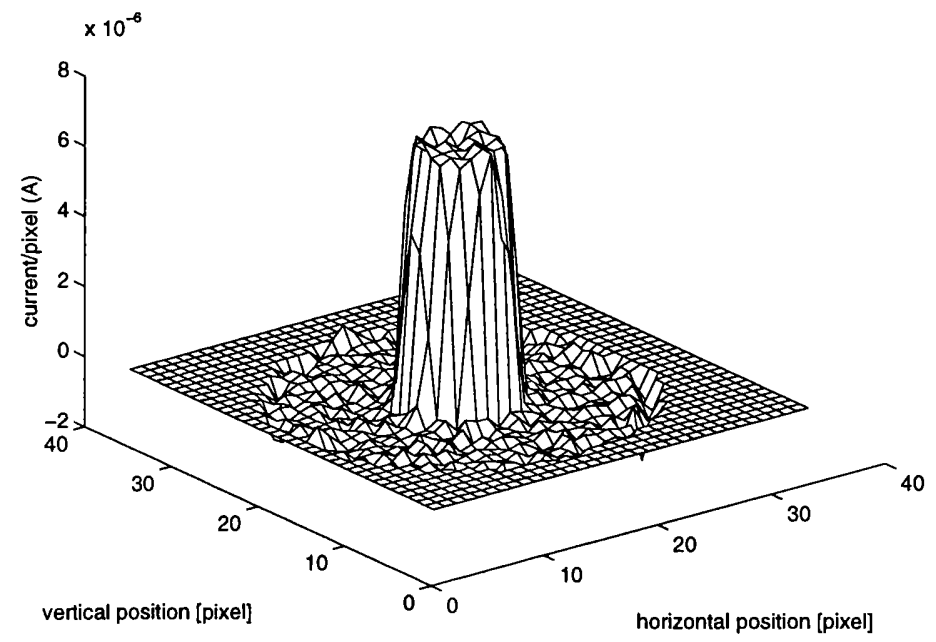

Figure 4.20-Surface plot of the current image computed from the magnetic field measured in the NMR experiment. This experiment was performed keeping the connecting wires parallel to the main field.

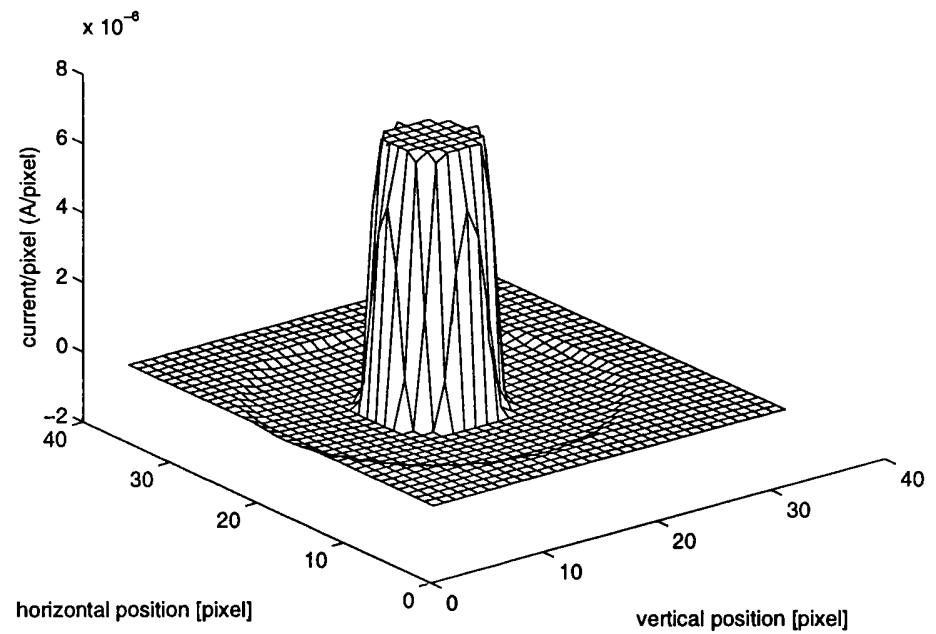

Figure 4.21-Surface plot of the current image computed only from the simulated magnetic field produced by the current in the inner cylinder.

The current image profiles are plotted in figure 4.22. The full line shows the current image profile obtained from the NMR experiment. The dashed line shows the current image profile calculated using the cylinder field only, and the dotted line is the profile of the current image calculated using the field of the cylinder plus the wires around it. The dot profile is the right one since the current image was computed from 
the total magnetic field, and not just the cylinder field.

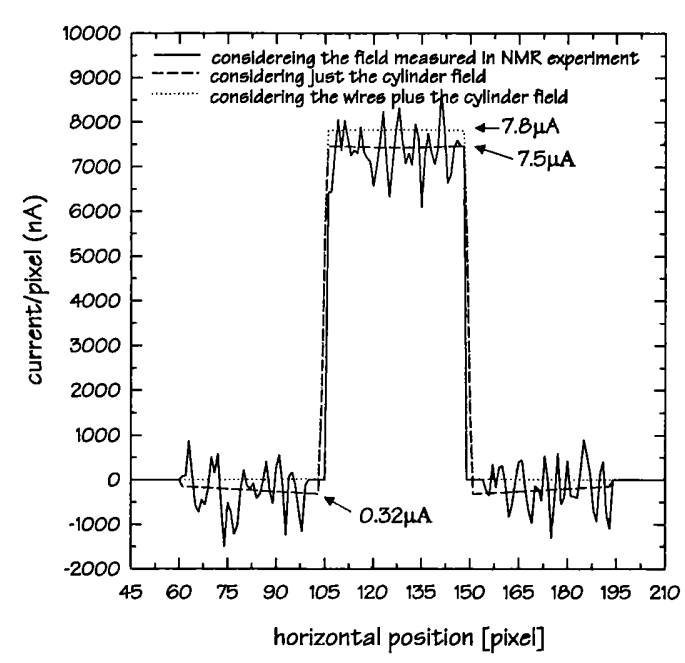

Figure 4.22- Horizontal profiles of the current images from: the field measured in the NMR experiment (full line), simulated cylinder field (dashed line), and simulated cylinder plus wires (dot line)

As seen in the surface plot (figure 4.21), in the outer cylinder region there is a very small negative current, which should not exist since all the current applied is confined to the inner cylinder region. Figure 4.22 detail shows the current images profiles. The dashed line is the profile of the current image calculated using just the numerically simulated cylinder field show this slightly lower current per pixel than the dot line. The full line profile in figure 4.22, obtained from the field measured in the NMR experiment show a better agreement with the dashed. This is because just the cylinder field was measured in the NMR experiment.

To quantify the error introduced in the current measurements, we performed a region surface integral to compare the total current applied with the current measured in the NMR experiment. Figure 4.23 shows the current images and the total current integrated per region. Figure 4.23(a) is the current image computed from the fields of the cylinder plus the wires around it, figure 4.23(b) is the current image computed from the cylinder field only and figure 4.23 (c) is the current image computed from the field measured in the NMR experiment.

For the complete magnetic circuit, figure 4.23(a), the total current integrated is 12.2mA, equal to the applied current, if the integrating region encloses both cylinders, and is equal to $12.0 \mathrm{~mA}$ if just the inner cylinder region is integrated. This difference is due to the same artifacts shown in figure 4.13. 

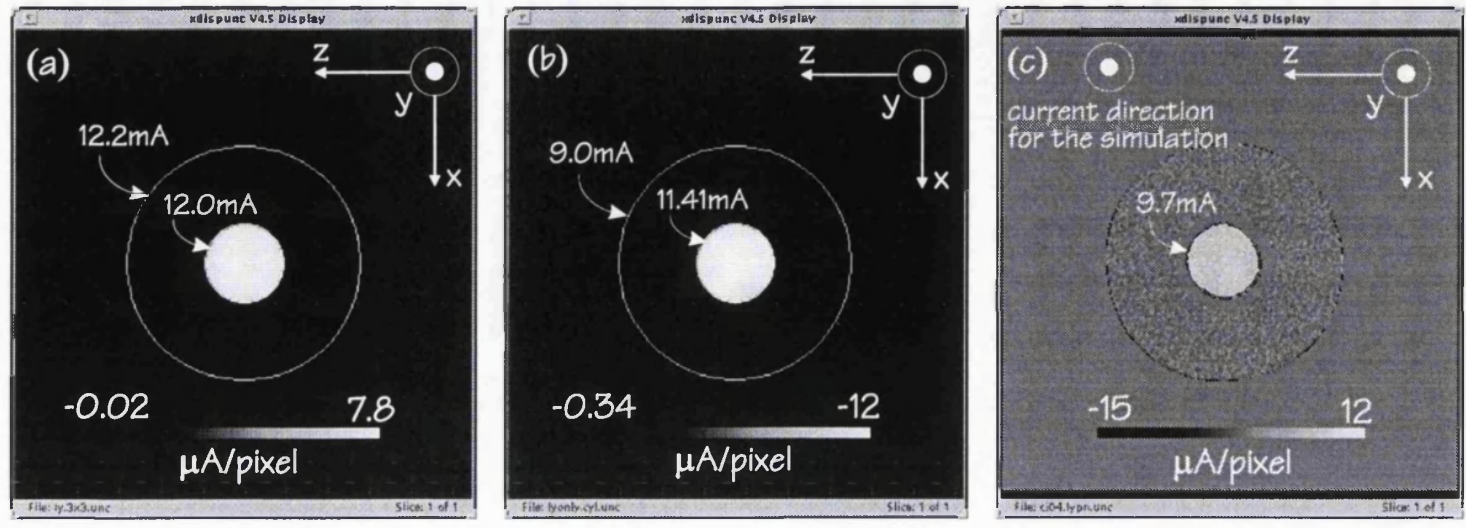

Figure 4.23-Current images calculated from (a) cylinder plus wires field $(b)$ just the cylinder field (c) from the field measured in the NMR experiment.

In the current image calculated only using the cylinder field the integrated values are equal to $9.0 \mathrm{~mA}$ and $11.41 \mathrm{~mA}$, respectively for the outer contour and inner contour. The $9.0 \mathrm{~mA}$ is due to the negative sum of the current in the outer cylinder which subtracts from the current integrated in the inner cylinder. The $11.41 \mathrm{~mA}$ value instead of the $12.2 \mathrm{~mA}$ which is the actual current applied, can be associated with the artifact shown in figure 4.13 plus the problem of applying Ampere's law to a short element of current. Therefore the difference between the current computed using the complete magnetic circuit and that just from the cylinder is equal to $0.59 \mathrm{~mA}$.

For the current image calculated from the field measured in the NMR experiment, just the integrated value of the inner cylinder is given since the rest of the image is just noise, the current integrated is equal to $9.7 \mathrm{~mA}$. This difference may be associated with two factors. Firstly with the area lost during the process of current calculation and secondly with fact that just the cylinder field was measured in this experiment. The area of the region used to integrate the current is equal to $466.92 \mathrm{~mm}^{2}$ and the actual inner cylinder area is equal to $535.02 \mathrm{~mm}^{2}$ (inner diameter $26.1 \mathrm{~mm}$ ), thus the area lost is equal to $68.1 \mathrm{~mm}^{2}$. Considering a uniform current density equal to $22.8 \mu \mathrm{A}$ $\left(12.2 \mathrm{~mA} / 535.02 \mathrm{~mm}^{2}\right)$, the current not integrated is equal to $1.55 \mathrm{~mA}$, which adds to the current integrated of $9.7 \mathrm{~mA}$ given a total of $11.25 \mathrm{~mA}$. Therefore the difference between the current applied and the current measured in the NMR experiment is equal to (12.2$11.25=$ ) $0.95 \mathrm{~mA}$. Part of this difference is due to the fact that the magnetic field of the wires was not measured. This introduces a difference of $0.59 \mathrm{~mA}$ as explained above, and the remaining difference between the current measured by the NMR experiment and that 
applied is equal to $(0.95-0.59=) 0.36 \mathrm{~mA}$, which can be associated with the intrinsic NMR experiment measurement error due to the noise.

To quantify the level of noise in the current image for this NMR experiment and compare this with the error introduced by using just the cylinder magnetic field, a noise analysis was performed as before. Two regions of 10x10 pixels, one in the inner cylinder and another in the outer cylinder were selected to measure the noise in the magnitude images. Both NMR magnitude images acquired before and after the object rotation were measured. Table 4.2 shows the noise analysis results which are in good agreement for the two orientations of the cylinder. The predicted mean standard deviation inside and outside the inner cylinder are $0.58 \mu \mathrm{A} /$ pixel and $0.63 \mu \mathrm{A} /$ pixel respectively.

Table 4.2 - Noise analyses results to quantify the standard deviation in the current image.

\begin{tabular}{|c|c|c|c|c|c|c|c|c|}
\hline \multirow{2}{*}{ region } & \multirow{2}{*}{ image } & \multicolumn{2}{|c|}{ magnitude } & \multicolumn{2}{|c|}{ phase (degree) } & \multirow{2}{*}{$\begin{array}{l}\sigma(B) \\
(n T)\end{array}$} & \multirow{2}{*}{$\begin{array}{l}{ }^{*} \sigma(I) \\
(\mu A)\end{array}$} & \multirow{2}{*}{$\begin{array}{l}{ }^{*} \sigma(I) \\
(\mu A)\end{array}$} \\
\hline & & $\sigma(M)$ & $<M>$ & ${ }^{*}<\sigma(\varphi) / M>$ & ${ }^{* *} \sigma(\varphi)$ & & & \\
\hline \multirow{2}{*}{ in } & $x / / B$ & 17.50 & 376.40 & 2.67 & 4.03 & 0.513 & \multirow{2}{*}{0.536} & 0.586 \\
\hline & $z / / B$ & 16.80 & 363.58 & 2.66 & 3.65 & 0.510 & & 0.583 \\
\hline \multirow{2}{*}{ out } & $x / / b$ & 14.71 & 360.00 & 2.35 & 3.30 & 0.451 & \multirow{2}{*}{0.462} & 0.515 \\
\hline & $z / / b$ & 17.65 & 307.87 & 3.30 & 3.45 & 0.634 & & 0.724 \\
\hline
\end{tabular}

" measured values "** predicted values

\subsection{Discussion}

In the first analytically solved simulation, because the calculated current image showed a non uniform distribution, we concluded that the simulated field was not completely correct. It would have been difficult to realise this problem by considering just the field images shown in figures 4.6 and 4.7 and also with the profiles in figure 4.8 , and for this reason we have decided to leave in the thesis this introductory part of the simulation, even though it is not completely right. The correct simulation is given in section 4.5 , where the magnetic field produced by the electrical current in the inner cylinder is numerically solved.

At the beginning of this $\mathrm{PhD}$ project, it was thought to be very important to keep the wires that connected the current source to the object being imaged always parallel to the main NMR field. This procedure would avoid any interference from the magnetic 
field due to the wires on the field measured inside the object. However, during the simulations described here we realised that this idea was not correct as subsequently proven in the second simulation. The NMR experiment performed by keeping the wires parallel to the NMR field produced results which agree with just the numerically simulated field produced by the current flowing in the cylinder, but which do not agree with the theoretical result expected when the full magnetic circuit is considered (cylinder plus wires). Although there are differences in the results they are very small, and can only be seen for the cylindrical phantom which has a uniform and predictable current distribution.

Comparing the profiles in figure 4.22 , the right current per pixel is $7.8 \mu \mathrm{A}$ (inner cylinder area $535.01 \mathrm{~mm}^{2}$ (diameter $26.1 \mathrm{~mm}$ ), pixel area $0.34 \mathrm{~mm}^{2}$ (fov $150 \mathrm{~mm}$, image matrix 256), current applied $12.2 \mathrm{~mA}$ ) while the dashed line in this figure shows $7.5 \mu \mathrm{A} /$ pixel. A difference of $0.3 \mu \mathrm{A} / \mathrm{pixel}$. The noise standard deviation in the current image is equal to $\pm 0.54 \mu \mathrm{A}$ (table 4.2). Therefore for the cylindrical phantom case, the error introduced by not measuring the wires field is within the predicted error bars.

Now, in the piglet head experiments performed prior to this simulation, the current standard deviation for the lowest noise experiment is $\pm 1.24 \mu \mathrm{A} / \mathrm{pixel}$ (table 7.7), which is at least twice the $\pm 0.54 \mu \mathrm{A} /$ pixel of the cylindrical experiment. Hence, the effect of not measuring the field due to the wires in the experiments may not be significant in the resulting current image distribution. However, it is better to measure their field and this was done for the live piglet experiment.

\subsection{Conclusion}

Two simulations and experiments were performed to validate the NMR technique to measure the electrical current. During the simulation development some aspects concerning the procedure used to perform the NMR experiments prior to this work were reviewed. The main point that arose is that to correctly measure the electrical current using the NMR technique, it is important to correctly place the wires that connect the object being imaged to the current source. They must be kept fixed to the object so that they can be rotated together and have their field measured. Even though the current in the wires does not contribute to the actual current being measured, their fields contribute to complement the magnetic circuit and allow Ampere's law to be applied correctly. Otherwise the current image distribution may contain small errors. 


\section{Chapter 5}

\section{Phantom experiment}

\section{Phantom experiments}

\subsection{Cubic phantom experiment}

In this chapter the results of an experiment performed to validate the algorithm used to calculate the $B_{y}$ field component, and to test the NMR image protocols used for the in vivo experiments is presented. As explained in section 3.4, to perform the current measurement experiment it is necessary to rotate the object by $90^{\circ}$. Since the NMR magnet has a small bore, the angle of rotation that can be achieved in the in vivo experiment is limited to a maximum of $45^{\circ}$ (because of the size of the piglet body). Hence, the actual component measured during the NMR experiment is a product of two components of current (section 7.2.1.4), and a post processing algorithm is required to determine the $B_{y}$ component.

The experiment was performed with a cubic phantom which was placed inside the magnet at the same positions planned for the in vivo experiment (chapter 8). For this the cube was placed inside the frame shown in figure 7.1, although the ceramic points were removed and the nylon-screws adjusted to press against the cube walls to hold it in position.

Since the results of a simulation with a cylindrical phantom have already been given in chapter 4 , the analyses in this experiment are limited to a comparison between the total current measure and the current applied during the experiment, and on verifying the current and magnetic field directions using the "right-hand" rule.

\subsubsection{Phantom description}

The initial experiments to measure the electrical current were performed with two different cubic phantoms. The first was a simple water tight cube with a filling hole in the top enabling it to be filled with the $\mathrm{CuSO}_{4}$ solution. The second cube was designed to be dismantlable to enable different objects to be placed inside it, thereby modifying the electrical current distribution. Although two cubes were constructed only results from experiments with the second cube are presented here.

Figure 5.1 illustrates the cubic phantom constructed to produce a non-uniform three-dimensional current distribution. It is constructed from perspex of $10 \mathrm{~mm}$ thickness 


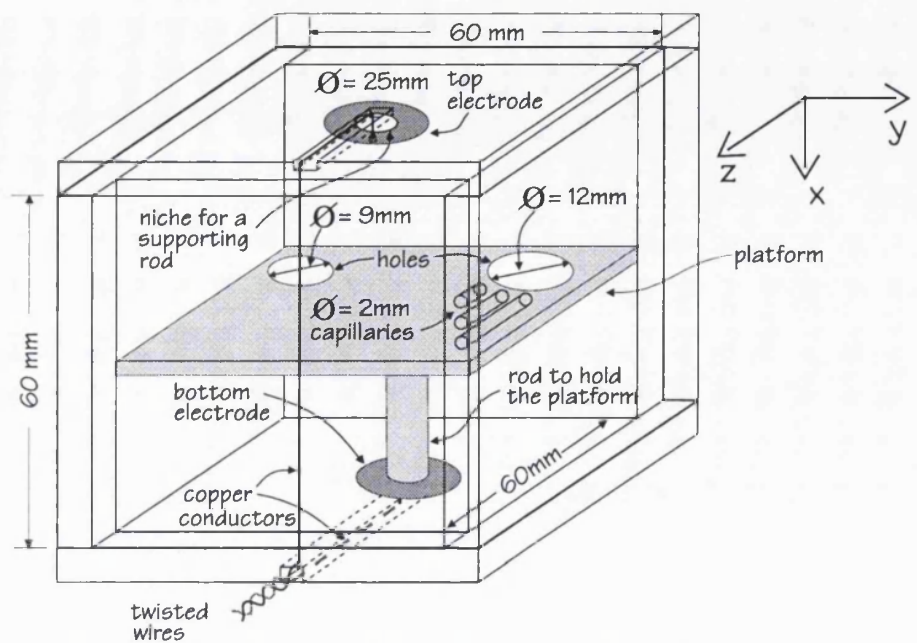

Figure 5.1 - Sketch of the cubic phantom constructed to produce a non-uniform current distribution and its axis reference.

and the internal size is $60 \times 60 \times 60 \mathrm{~mm}$. The six pieces $(2$ pieces of $80 \times 80 \mathrm{~mm} ; 2$ pieces of $80 \times 62 \mathrm{~mm}$ and 2 pieces of $60 \times 62 \mathrm{~mm}$ ) were cut and machined to the right size. On the top side a recess of $3 \mathrm{~mm}$ was machined to hold a rubber seal ( $1 \mathrm{~mm}$ thickness). The top side is fixed onto the cube with six nylon-screws. To provide support for whatever object is intend to be placed inside of the cube, a circular cavity of $4.0 \mathrm{~mm}$ was machined on the inside surface of the top and bottom sides. These holes can be used to locate and hold a supporting rod. Also on the top and bottom, a circular cavity $1.0 \mathrm{~mm}$ deep was machined to hold the silver electrodes in form of annuli with external and internal diameters of $25 \mathrm{~mm}$ and $10 \mathrm{~mm}$ respectively. The electrodes were electrically connected to the outside by a $1.0 \mathrm{~mm}$ silver wire.

The electrodes are made of $1 \mathrm{~mm}$ thick silver sheet coated with silver-chloride, with the silver-wires joined with silver-solder on the back. The silver-solder is required to avoid possible corrosive effects in the contact. Silicon-rubber was used to stick the electrodes in position. This avoids water leaking through wire hole that goes through the wall of the cube. To minimize any disturbance of the main magnetic field near to the cube, pure copper wires were used as the connecting wires coming from the current source.

The object placed inside the cube to make the current inside of it non-uniform is a platform with two holes of 9 and $12 \mathrm{~mm}$ diameter. The platform is made of $2 \mathrm{~mm}$ perspex and is held at the centre of the cube with adhesive tape and a supporting rod. Since the platform was a tight fit inside the cube, nothing special was done to prevent 
the electrical current going through the small gaps that may exist between the cube wall and the platform edges.

The copper conductors at the top and bottom sides of the cube lay in $2 \times 2 \mathrm{~mm}$ machined slots, and the returning wire from the top electrode ran along the cube wall. The conductors were held in position with cyanoacrylate adhesive. The power supply was then connected to these conductors through a pair of twisted wires, as in the cylinder phantom simulation experiment.

To distinguish the image slices three capillaries of $2 \mathrm{~mm}$ diameter but different lengths and separated from each other by $3 \mathrm{~mm}$ centre to centre, were drilled in the cube wall. The centre of the first capillary is $6 \mathrm{~mm}$ from the centre of the platform and is $32 \mathrm{~mm}$ long. The second and third are $24 \mathrm{~mm}$ and $17 \mathrm{~mm}$ long.

The salt solution used to fill the cube was $0.9 \mathrm{~g} / \mathrm{dl}$ of $\mathrm{NaCl}$ with $0.25 \mathrm{~g} / \mathrm{dl}$ of $\mathrm{CuSO}_{4}$, which gives a $\mathrm{T}_{1}$ and $\mathrm{T}_{2}$ approximately equal to $100 \mathrm{~ms}$.

\subsubsection{NMR image parameters and electrical current}

The spin-echo sequence used for this experiment was set to a repetition time of $600 \mathrm{~ms}$, echo-time of $60 \mathrm{~ms}$, slice thickness $3 \mathrm{~mm}$ and field of view of $16 \mathrm{~cm} .256$ by 256 image matrix pixels of 0.63 by $0.63 \mathrm{~mm}$ were used in both transverse and coronal slices. The slice positions are shown in figure 5.2(b), the bottom, centre and bottom slices are at $6 \mathrm{~mm}, 9 \mathrm{~mm}$ and $12 \mathrm{~mm}$ from the centre of the platform. The scan time for each image in these experiment was $3 \mathrm{~min}$ and $10 \mathrm{sec}$.

The amplitude of the current pulses was set to $60 \mathrm{~mA}$, divided into a positive and a negative pulse each of $30 \mathrm{~ms}$ duration. During the refocusing pulse the current was switched off for $4 \mathrm{~ms}$. The current was applied in the $x$-direction of the axis orientation as shown in figure 5.1, going from the top to the bottom electrode.

\subsubsection{Results}

In figure 5.2(a) are shown the NMR magnitude images before registration with no current and showing the axis orientation in relation to the main field $\boldsymbol{B}_{\boldsymbol{0}}$. This figure is divided into three sets (or columns in the figure), each set corresponds to one orientation of the cube in relation to the main field and each set is composed of three different slice positions called bottom, centre and top slices (rows in the figure). All tomographic planes are parallel to the $y z$-plane. In figure 5.2(b) the position of the three slices in relation to the platform is shown. In figure 5.2(a) the capillaries lengths defines 
the slice position. In the top slice the capillaries did not have enough contrast, probably because of poor filling with $\mathrm{CuSO}_{4}$ solution. The dots in these image are the capillaries in the columns of the frame built to hold the piglet during the experiments (see section 7.1.1.1 for details of the frame design). Just three of the four columns had their capillaries filled with $\mathrm{CuSO}_{4}$ solution. The position of the empty capillary is the origin of the object axes reference. Also note the difference in the $x$-axis orientation between that in figure 5.1 and that of the images in figure 5.2.

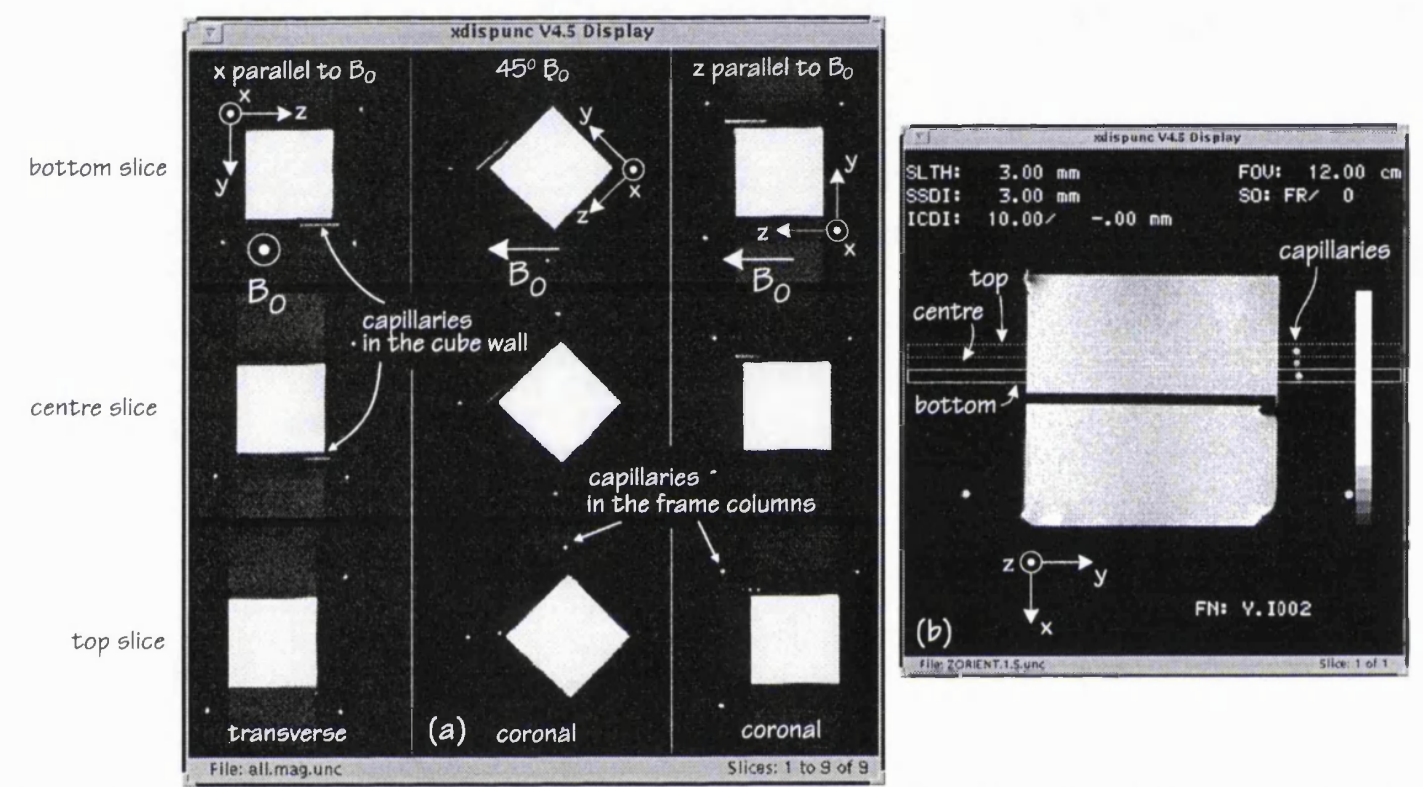

Figure 5.2-(a) Slices of the cubic phantom to show the image orientations in relation to $B_{0}$, and (b) a transverse slice to show the image positions in relation to the platform.

\subsubsection{Electrical current magnetic field}

In figure 5.3 the calculated magnetic field from the NMR phase unwrapped images at the centre slice position are shown together with the axis orientations and the applied current direction during the experiment. In this figure the top row are the field components in the $x$-direction, centre row in the $y$-direction and bottom row in the $z$ direction. The axis that was parallel to $\boldsymbol{B}_{0}$ during the image acquisition is also indicated on the left-hand side of this figure. The magnetic field in the y-direction is the result of the field calculation as described in section 7.2.1.4.

In figure 5.3 the black regions represent the negative field and white positive, and they are consistent with the predictions given by the "right-hand" rule. Consider, for instance, the $B_{z}$ component which is the bottom row in figure 5.3. This component 


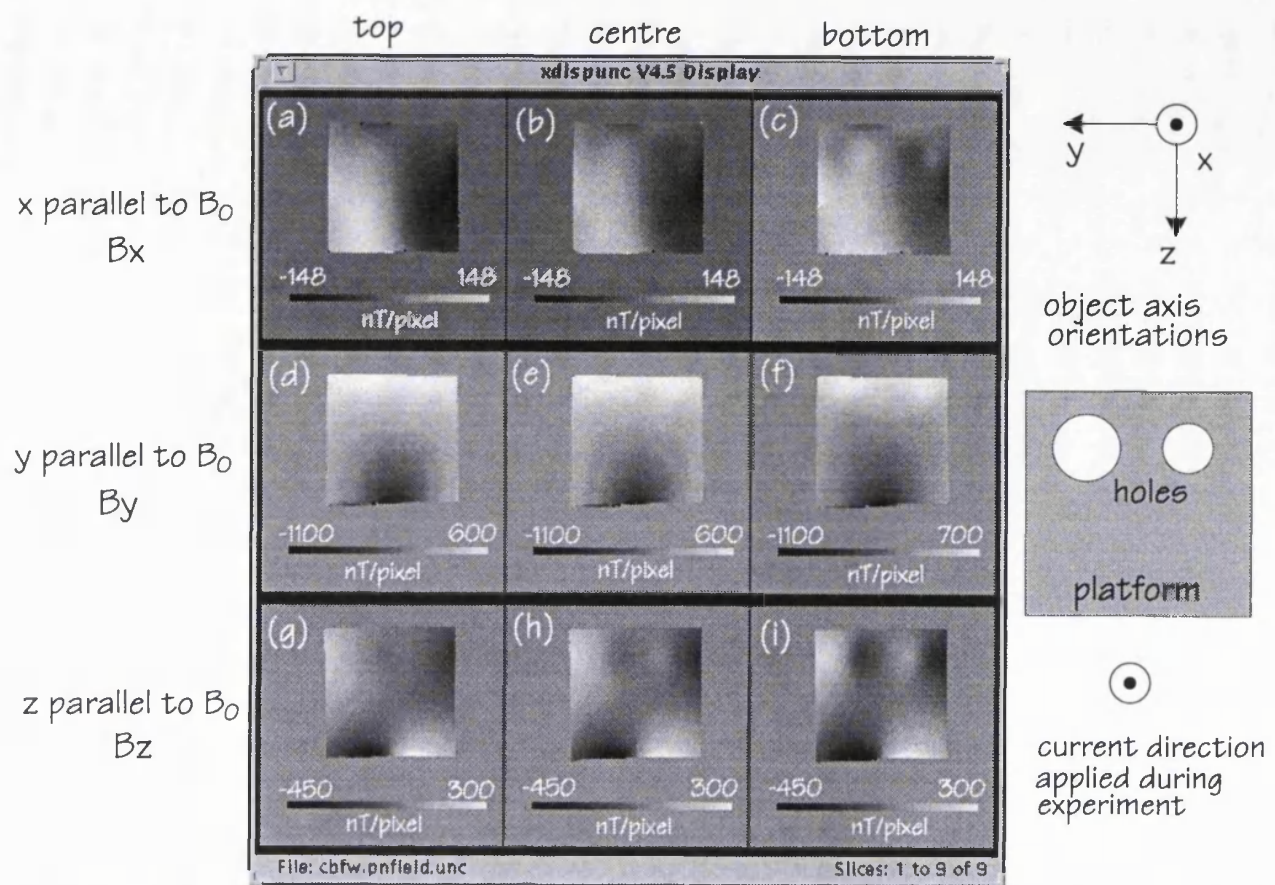

Figure 5.3 - Current magnetic field in the $x$-, $y$-and z-direction at the three slice positions and a sketch of the platform inside of the frame with the object axis orientation.

shows that the magnetic field due to the current inside the cube and that from the current in the wire running along the outside wall of the cube. The current through the holes is coming out of the paper plane producing the white and black regions on the right and left side of the platform, which can be verified by the "right-hand" rule. The same thing happens with the return current wire field, where now the current is going into the paper plane and therefore produces fields in the opposite direction. The magnetic field along the slice plane can be better visualised with the vector plot shown in figure 5.4. To plot the vectors the $B_{y}$ and $B_{z}$ components shown in figure 5.3, centre
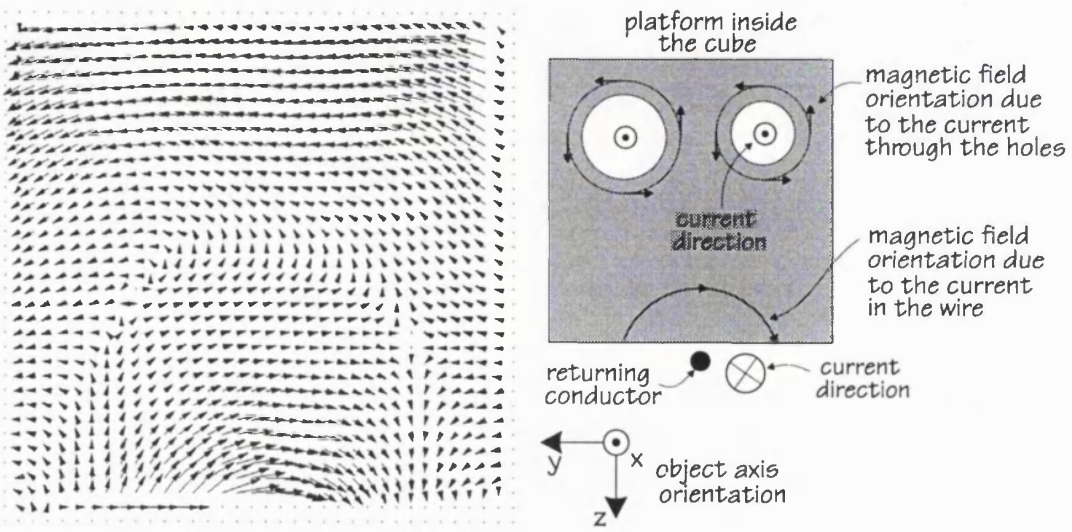

Figure 5.4 - Vector plot of the current induced magnetic field, and a schematic of the platform inside of the cube with the axes orientation. 
slices, were used and the number of vectors in the ROI was reduced with a 3 by 3 mask to average the signal intensity.

The vector plot shows that in the region between the holes there is no zcomponent of the magnetic field. This is because the holes split the current into two parts, mainly oriented in the $x$-direction, and the fields produced in the $z$-direction by each part of current nearly cancel each other.

Another aspect of the vector plot is the field direction in the central region of the cube. The vector plot shows that the field in this region is oriented in the $+y$-direction, against the direction given by the "right-hand" rule when just the direction of the current through the roles is considered (see draft in figure 5.4). The explanation of this relies on the -z-current component shown in figure 5.6(b) which has a reasonably high value and hence determines the field direction in this region.

\subsubsection{Current images}

Figure 5.5 shows the images of the electrical current component in the $x$ direction in the three adjacent tomographic planes (a) is in the top, (b) is in the centre and (c) is in the bottom slice. In this experiment just the positive current is shown, because only the NMR data with a positive-negative current sequence was acquired.
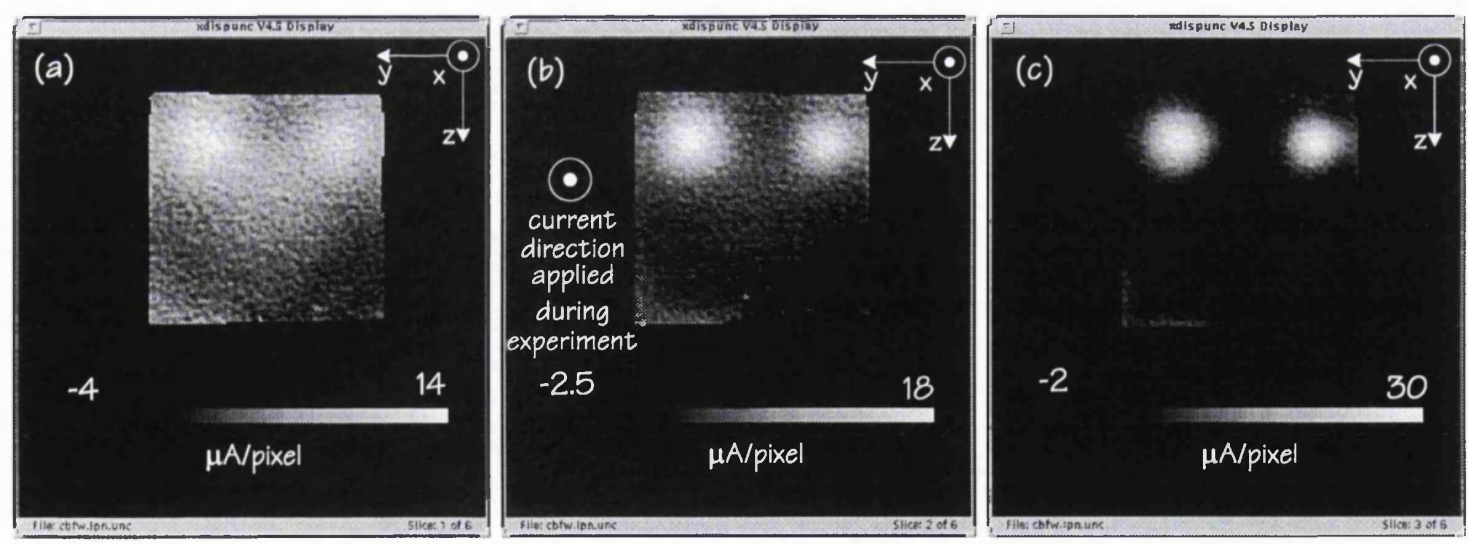

Figure 5.5 - Component of current in the x-direction. (a) top slice, (b) centre slice and (c) bottom slice.

In figure 5.5, even though the total amount of current passing through each slice is the same, the contrast in the three images has been set to different ranges. The reason for this is that the current per pixel is larger in the slices near to the holes in the platform.

The total current crossing each slice was integrated by defining a contour line 
around the $I_{x}$ images shown in figure 5.5. In the top slice the total current integrated was $53.41 \mathrm{~mA}$, in the centre slice $52.46 \mathrm{~mA}$ and in the bottom slice $52.44 \mathrm{~mA}$. Considering that a total of $60 \mathrm{~mA}$ was applied during the experiment the overall average of the integrated current gives an error of $12.6 \%$, which is within the expected error as explained in the conclusion of the noise analyses, section 6.6.

In figure 5.6 the electrical current components in the $y$-and $z$-direction are shown together with the current magnitude image at the central position of the three slices. The magnitude is the calculated vector sum of figures 5.5(b) and 5.6(a) and (b).
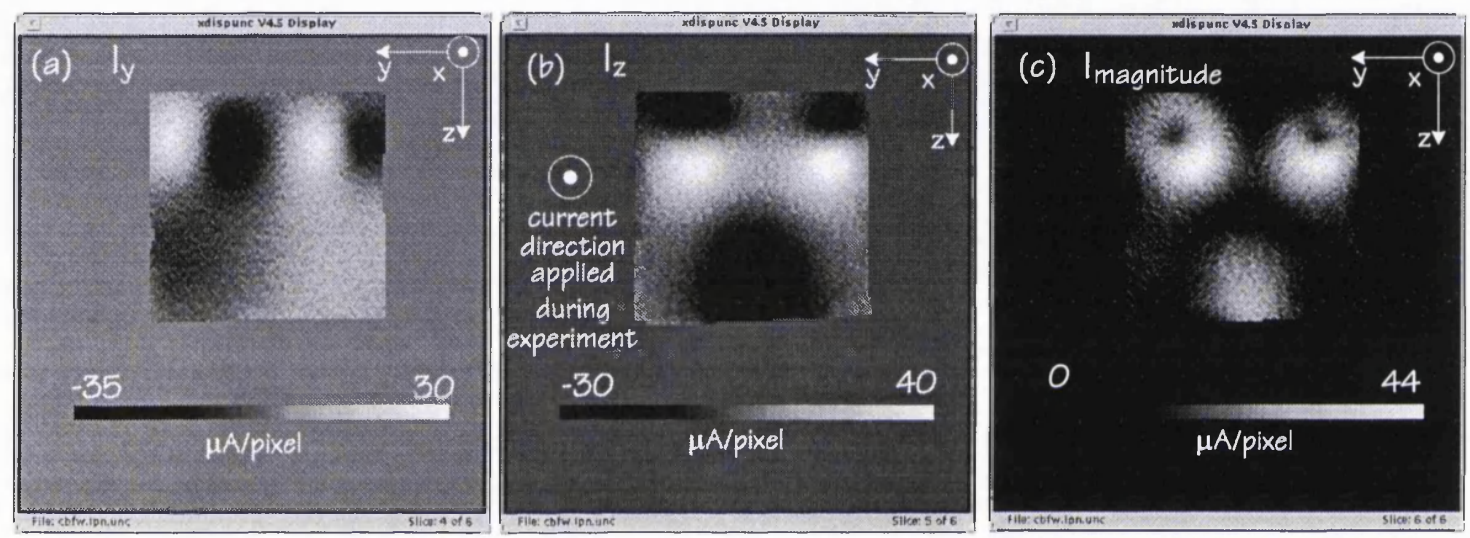

Figure 5.6 - Current components in the y-direction (a), z-direction (b) and magnitude (c).

Using the $I_{y}$ and $I_{z}$ components, figure 5.6(a) and (b), the vector plot shown in figure 5.7 was produced. To plot the vectors the number of pixels in the ROI was reduced with a 3 by 3 mask to average the signal intensity. A program was implemented in $\mathrm{C}$ to perform this task and also to store the averaged data in a format suitable for

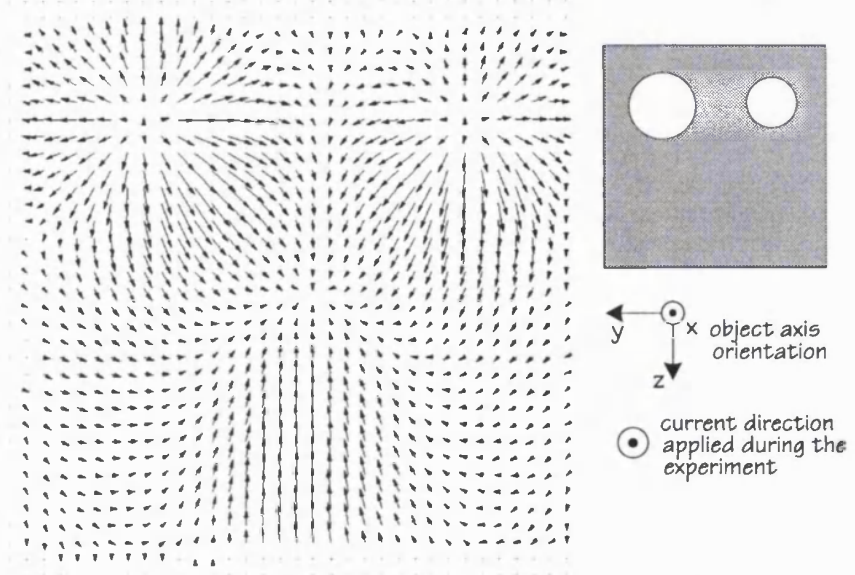

Figure 5.7 -Vector plot of the current in the tomographic plane, and a sketch of the platform to show the holes position and object axis orientation. 
"Mathematica" which was used to plot the vector images.

\subsubsection{Discussion}

The current images and the vector plots show that even for this simple, uniform and isotropic medium the electrical current is not just restricted to the immediate surroundings of the holes in the platform, on its route from the top electrode to the bottom electrode. Instead it spreads throughout the upper side of the cube, going as far as to the opposite side of the holes in the platform (see the $I_{z}$-component in figure 5.6(b)). The three images of the $I_{x}$ component in figure 5.5, show that at the position of the slice nearest to the platform, practically all the $x$-current is concentrated in the holes whereas in the top slice it is more spread across the image. In figure 5.5 and in the magnitude image figure 5.6(c) it is possible to visualize the difference in the current density due to the holes diameter, which is higher in the right-hand side where the hole is smaller.

\subsubsection{Conclusion}

In this section an experiment to measure a non-uniform current distribution inside a cubic phantom was described. The experiment was performed with the aims of firstly creating the NMR image protocols to be used in the in vivo experiment and secondly to validate the routine implemented to compute the $B_{y}$ field component from the small angle of rotation. The results of the total current integrated in $x$-direction, which depends on the algorithm implement to project the field measured in the $y$-direction, gave an error of about $12 \%$ which is within the expected error as concluded in Chapter 6.

\subsection{Experiment performed with a EIT tank phantom}

This section presents results of some experiments performed to verify the contrast resolution of the current image, i.e., the minimum current difference between two regions that can be measured. The idea is to increase the resistivity in the central region of a "conventional" EIT tank phantom specially constructed for this experiment by the addition of a rod of polyacrylamide gel or a synthetic sponge. Both of these materials have been fully tested for this purpose by the UCL EIT group (Holder et al. in press). The polyacrylamide gel increases the local solution resistivity from $10 \%$ to $200 \%$ depending on the gel concentration (Holder and Khan, 1994), and synthetic sponges may increase the local solution resistivity by up to a maximum of $17 \%$ depending on the sponge density. The first experiment was performed with a polyacrylamide gel which 
increased the local resistivity by $10 \%$, and the second experiment was performed with a high density sponge which increased the local resistivity by $17 \%$. Because in the experiment with the gel no differences in the current image were detectable, just the results of the second experiment with the synthetic sponge are presented here. These experiments were performed with opposing electrodes being driven in order to maximise the current in the middle of the phantom, but as a preliminary study, one experiment was performed on a uniform phantom using adjacent electrode drive which is the most commonly used configuration in conventional EIT.

\subsubsection{Phantom description}

The EIT tank constructed for the experiments is similar to those used in many EIT experiments (Webster, 1990) and it is illustrated in figure 5.8. Basically it consists of a cylinder of perspex with inner diameter of $79 \mathrm{~mm}$ and length of $35 \mathrm{~mm}$. The cylinder is sandwiched between two 100 by $100 \mathrm{~mm}$ squares of perspex $5 \mathrm{~mm}$ thick. In the base a circular groove was machined into which the cylinder could be located and glued. The top is fixed to the cylinder with eight nylon-screws of $3 \mathrm{~mm}$ diameter, therefore the tank can be opened. To ensure a watertight seal, a rubber-seal $1 \mathrm{~mm}$ thick and $78 \mathrm{~mm}$ inner diameter is placed between the cylinder and the top square.

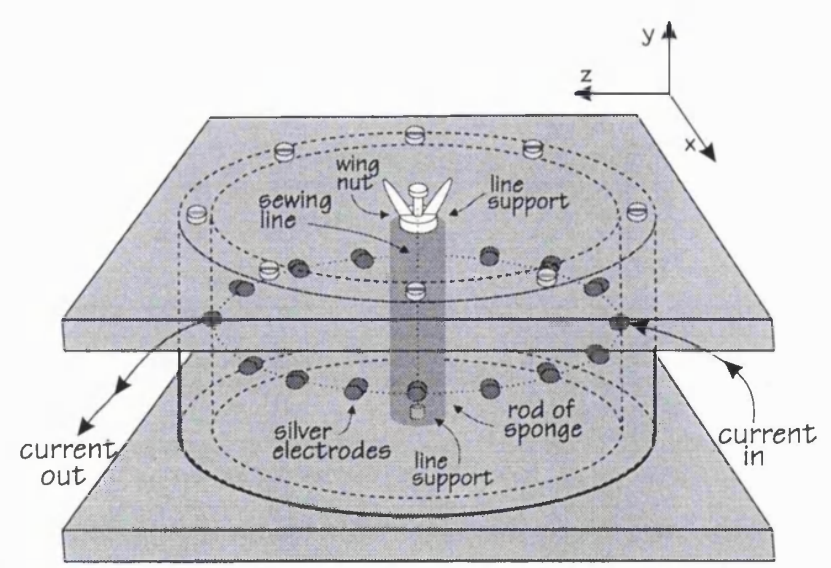

Figure 5.8 - EIT phantom

The $5 \mathrm{~mm}$ diameter electrodes were made of $1 \mathrm{~mm}$ thick silver sheet with silverwires joined on the back. These are fixed to nylon-screws of $4 \mathrm{~mm}$ diameter, the screws heads being removed to allow the electrodes be near to the cylinder wall. A $1 \mathrm{~mm}$ diameter hole along the length of the nylon-screw lets the silver-wire pass through it, 
the hole being sealed with silicon rubber. The screws are fixed to the cylinder wall through threaded holes and finally, from the outside of the cylinder, a rubber washer and a nylon-nut are used to avoid water leakage. A total of sixteen electrodes are symmetrically distributed around the middle of the cylinder.

\subsubsection{NMR image parameters and electrical current}

The spin-echo sequence used in this experiment was set to a repetition time of $600 \mathrm{~ms}$, echo-time $120 \mathrm{~ms}$, slice thickness $2 \mathrm{~mm}$ and field of view $12 \mathrm{~cm}$. 256 by 256 image matrix pixels of 0.47 by $0.47 \mathrm{~mm}$ were used in the coronal and transverse slices. All slices were acquired parallel to the $x z$-plane. The central slice was set at the tank centre and the top and bottom slices $2 \mathrm{~mm}$ and $-2 \mathrm{~mm}$ from this position. The scan time for each image in this experiment was $2 \mathrm{~min} 43 \mathrm{sec}$.

The current pulse amplitude was set to $18.3 \mathrm{~mA}$, divided into a positive and a negative pulse, each of $30 \mathrm{~ms}$. During the refocusing pulse the current was switched off for $4 \mathrm{~ms}$. The pair of electrodes selected for the application of the electrical current were the two diametrically opposed electrodes in the $z$-direction (figure 5.8), the reason for this choice is that this configuration gives a more uniform current distribution inside the tank.

\subsubsection{Results}

\subsubsection{Current density in the EIT tank with adjacent electrode drive}

The same NMR image parameters described in section 5.2.2 was used in this experiment. The electrical current pulse amplitude applied during the image acquisition was $6.1 \mathrm{~mA}$. Figure 5.9 shows images of the current images components $I_{x}, I_{y}, I_{z}$ together with the current magnitude $I_{\text {mag. }}$. Also indicated in this figure are the electrodes used to drive the electrical current.

The images can be easily analysed as follows. The black region shown by the $I_{x}$ component near to the electrode where the current goes into the tank $\left(I_{\text {in }}\right.$ in figure 5.9) is due to the current going in the negative direction of the $x$-axis. Next, the part of the current that keeps within the $2 \mathrm{~mm}$ slice thickness turns into the $-z$-axis which is indicated by the black region between the adjacents electrodes in the $I_{z}$ image. Finally this current turns into the $x$-direction to reach the other electrode and leaves the tank producing the white region in the $I_{x}$ image. The two white spots in the $I_{z}$ current component are due to the electrical current that goes deeper into the tank producing 

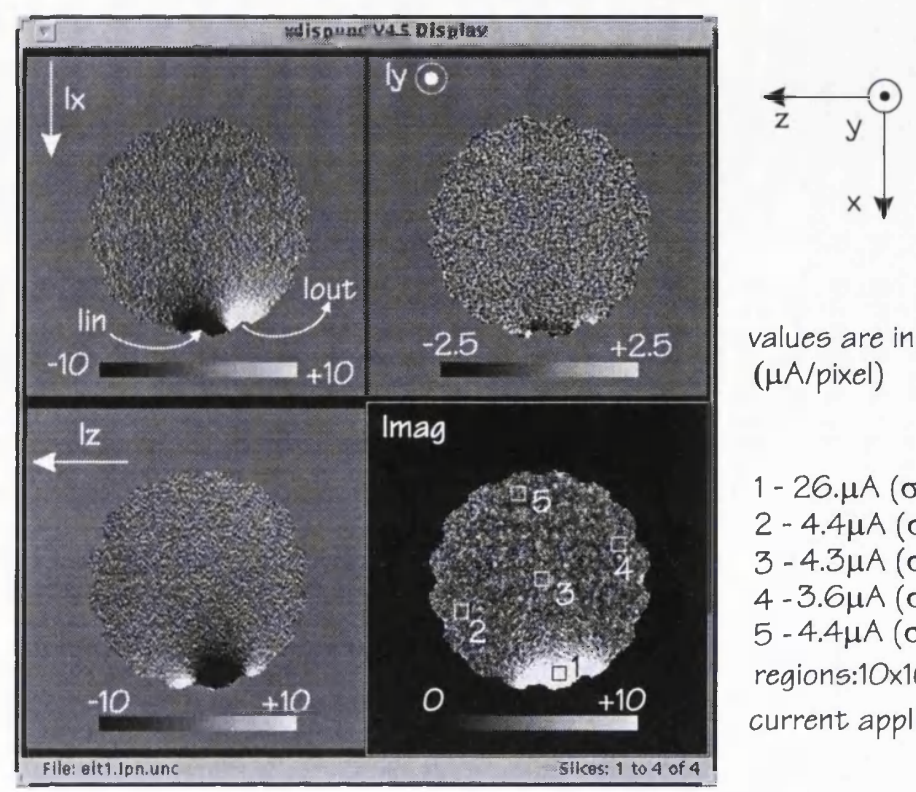

values are in: $(\mu \mathrm{A} /$ pixel)

$1-26 . \mu A(\sigma=7.4 \mu A)$

$2-4.4 \mu A(\sigma=1.9 \mu A)$

$3-4.3 \mu A(\sigma=1.9 \mu A)$

$4-3.6 \mu A(\sigma=1.9 \mu A)$

$5-4.4 \mu \mathrm{A}(\sigma=1.6 \mu \mathrm{A})$

regions: $10 \times 10$ pixels

current applied: $6.1 \mathrm{~mA}$

Figure 5.9 - Electrical current distribution inside the EIT tank using adjacent current drive electrodes.

components of current in the $+z$-direction at the outside edge of the electrodes. The $I_{y}$ component is much more uniform across the image, probably because the very small part of the current that goes out of the plane is hidder' by the noise in the image.

The $I_{m a g}$ image in figure 5.9 shows the total current in five different regions inside the tank. It is interesting to note that the electrical current more than a few millimetres from the electrodes is reasonable uniformly distributed across the tomographic plane.

\subsubsection{Current density in the EIT tank with opposite electrode drive}

Figure 5.10 shows (a) the NMR magnitude images acquired with current and (b) the corresponding NMR phase images, together with the axis orientations in relation to the main field $\boldsymbol{B}_{0}$, after the images were registered. These images are divided into three sets (or rows in the figure), each set corresponds to one orientation of the phantom in relation to the main field and each set is composed of three different slice positions called bottom, centre and top slices (columns in the figure). All tomographic planes are parallel to the $x z$-plane defined by the object axis, figure 5.8. The images in the centre column show the capillaries used to mark the central slice position of the phantom, which match the plane defined by the 16 electrodes placed around the phantom.

In figure 5.10(a) the lower NMR signal in the centre of the tank is due to the reduction of the water content in the region occupied by the synthetic sponge. This is the reason why the sponge is well defined in the centre of the NMR magnitude images. 


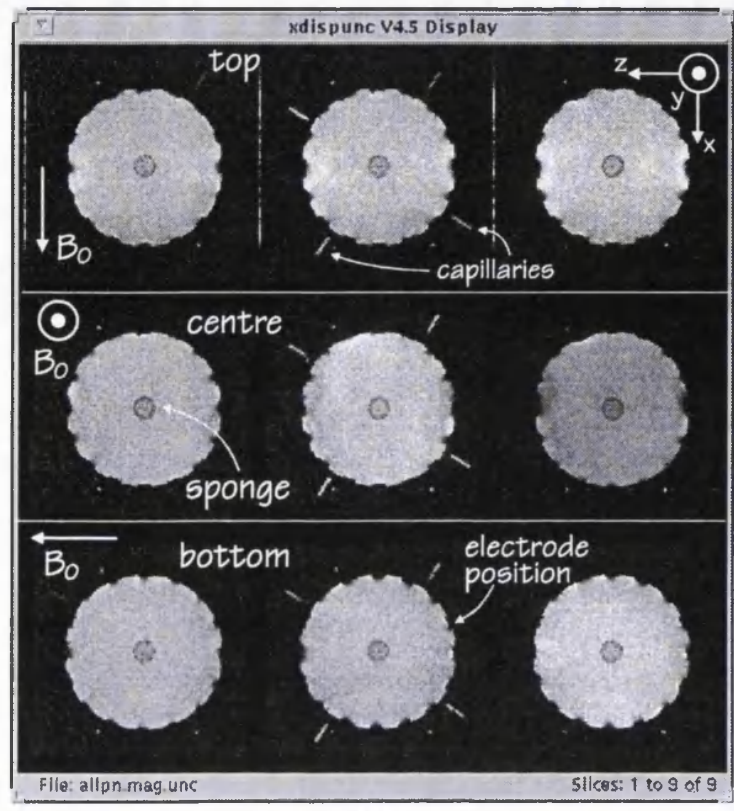

(a)

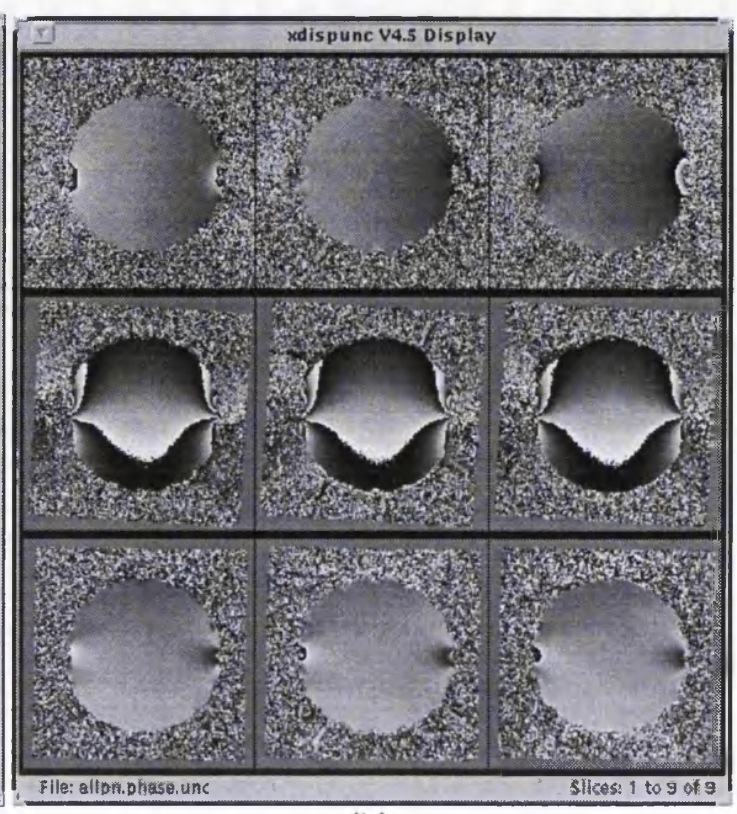

(b)

Figure 5.10 - (a) The NMR magnitude images and (b) the corresponding phase images of EIT phantom experiment.

On the other hand, because the synthetic sponge itself does not affect the main NMR field, and because the current changes produced by its presence are very small the phase images in figure $5.10(\mathrm{~b})$ are not able to show the sponge presence.

\section{Electrical current magnetic field}

Figure 5.11 shows the calculated magnetic field from the unwrapped NMR phase images at the centre slice position together with the object axis orientation and the current direction applied during the experiment. In this figure the image contrast had to be stretched to a range smaller than the range of actual image values, otherwise the central region of the phantom would not be easily visible. This procedure caused saturation of the regions near to the electrodes. Thus the average values of regions of 5 by 5 pixels around the image and near the electrodes are indicated in the figure.

Note that in this experiment the current is applied in the $+z$-direction which is along the centre of the image plane. Hence, the only field in figure 5.11 that can be easy verified using the "right-hand" rule is the $B_{y}$ component, which shows a darker region, or negative field, in the upper side of the image, and vice-versa. Note also that the values indicated in the magnitude of the field shown in figure 5.11, are as expected 

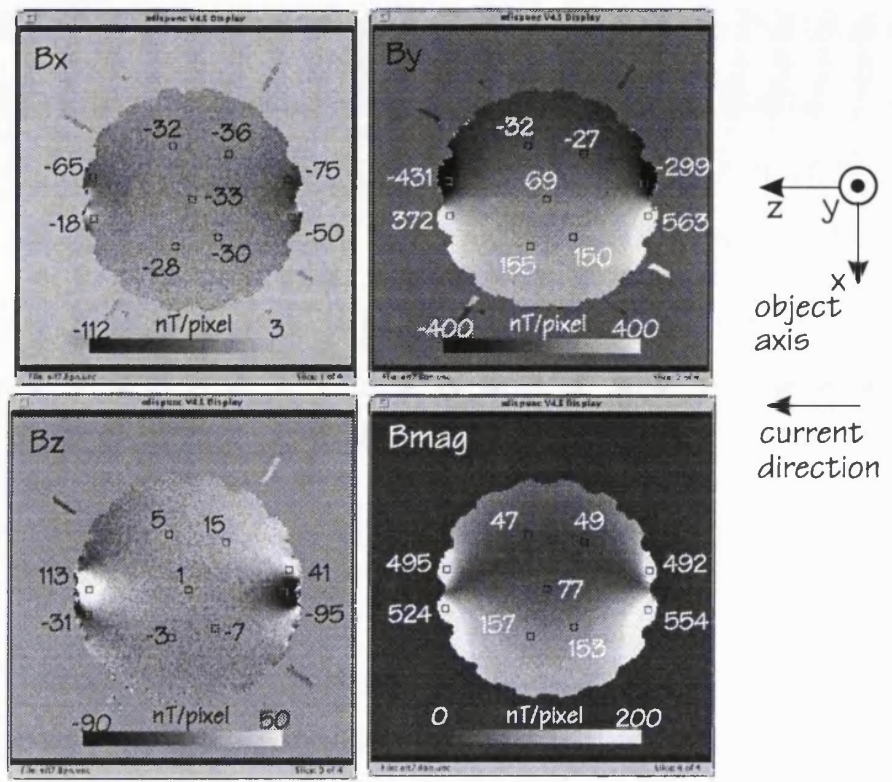

direction

Figure 5.11 - Magnetic field in the EIT phantom experiment at the central slice position

symmetrical because of the phantom symmetry.

\section{Electrical current images}

In figure 5.12 the images of the electrical current component in the $x-, y$ - and $z$ direction are shown together with the current magnitude image for the central slice. Again, in this figure, because the current density near to the electrodes is very high, the contrast in these regions is saturated and the scale bar does not include the values of the saturated regions, but the mean value of various regions of 5 by 5 pixels are again indicated in the images. In this experiment because the plane of the image is not
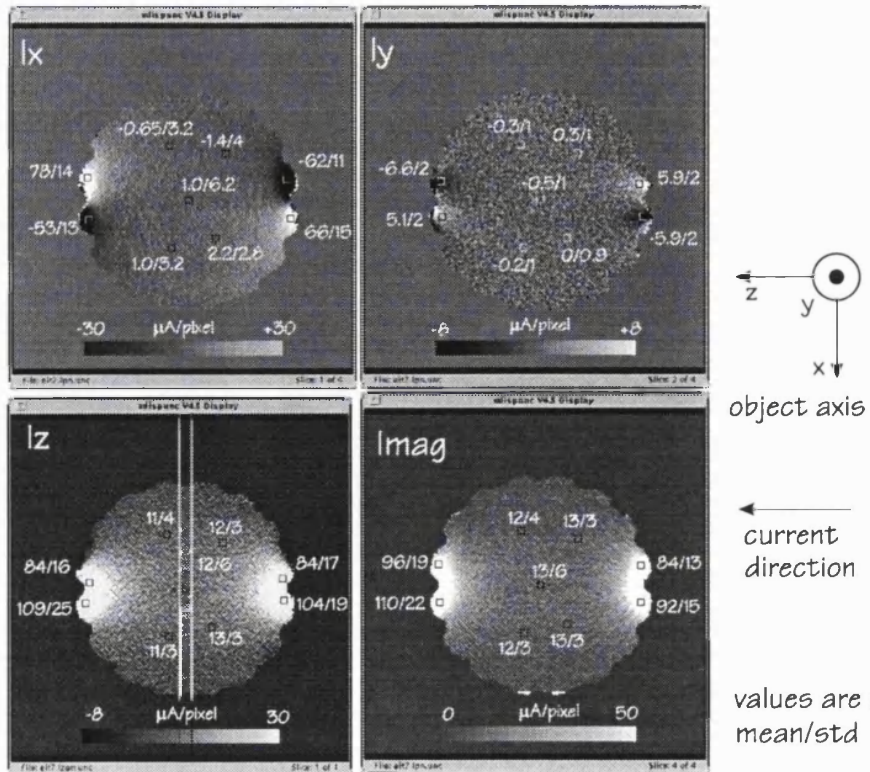

object axis

Figure 5.12 - Current images in the EIT phantom experiment 
transverse to the current direction it is not possible to quantify the total current measured and to compare it with the current applied during the experiment.

In the $I_{x}$ and $I_{y}$ components, figure 5.12, the four mean values indicated in the central region of the phantom are very low compared with the local standard deviation. This is because the current density in these regions is too small compared with the intrinsic noise level of the NMR system. However, near to the electrodes where the current density is higher the absolute values indicated are very similar. This symmetry is more remarkable in the $I_{z}$ and $I_{m a g}$ components and the reason is probably the phantom symmetry.

The $I_{z}$ current component in figure 5.12, only faintly shows the presence of the sponge in the centre of the phantom. This is confirmed by a vertical profile, 10 pixels width, taken across the centre of the image and which is shown in figure 5.13. A dip in the data indicates where the sponge is located. In this profile two horizontal lines have been drawn: the lower line indicates the mean value at the sponge site (approximately $11 \mu \mathrm{A})$, while the upper line shows the expected value in the central region if the sponge

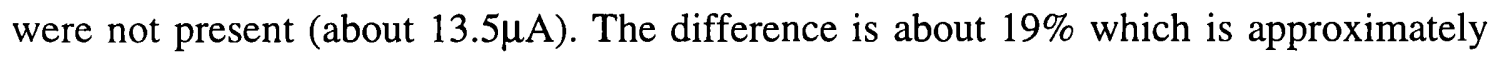
equal to the resistivity increase produced by the sponge as measured by the EIT group here at UCL (17\%).

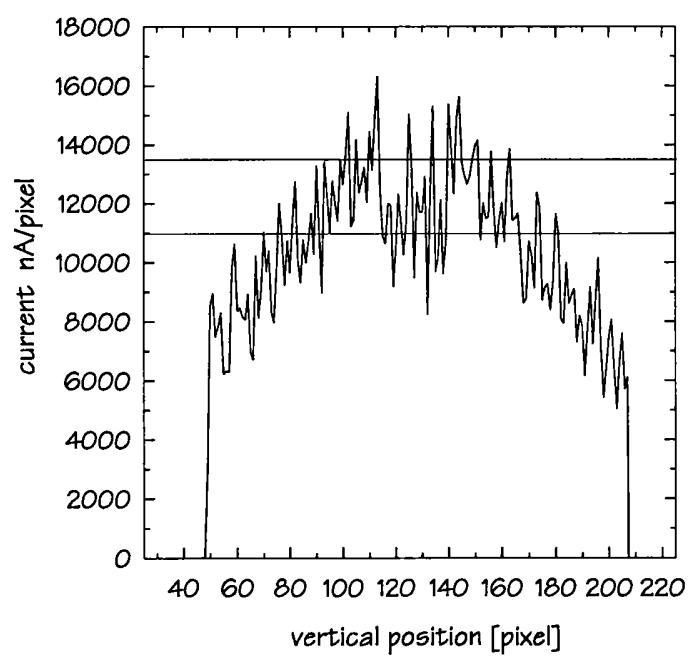

Figure 5.13 - Vertical profile, 10 pixels wide, of the Iz component shown in figure 5.12.

Figure 5.14 shows a surface plot of the $I_{z}$ component of current. Before the surface was plotted a 3 by 3 mask was applied to average the signal intensity and reduce 


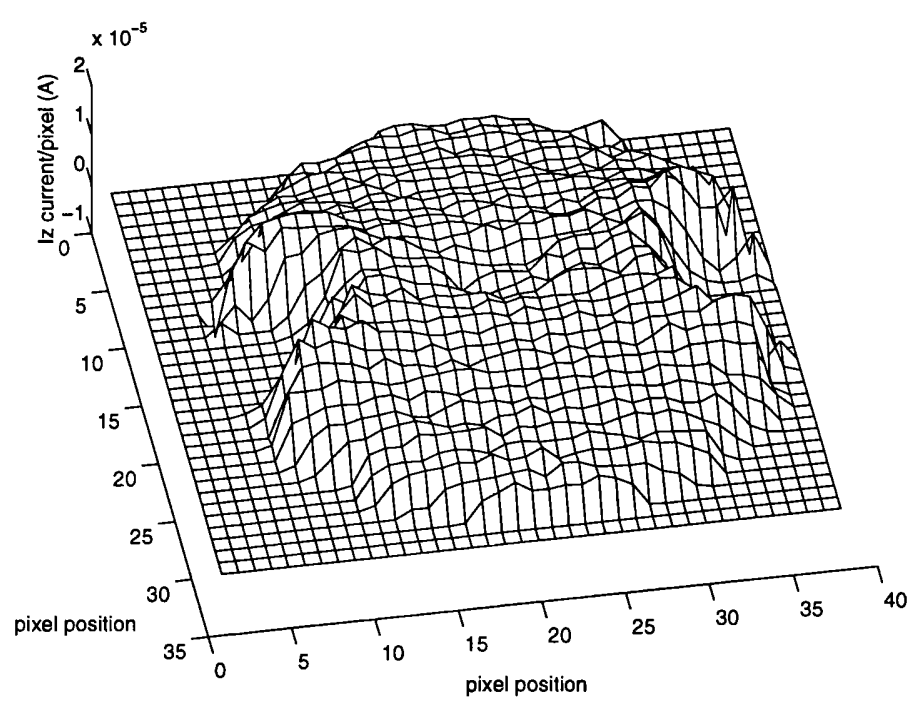

Figure 5.14 - Surface plot of the $I_{z}$ component of current.

the number of pixels. It is just possible to observe in the centre of the plot the dip produced by the slight reduction in the current per pixel due to the sponge presence.

\subsubsection{Discussion and conclusion}

The aim of the EIT phantom experiment was to verify experimentally the minimum contrast resolution in the current image that can be measured by the NMR current experiment. To perform this test it is necessary to place objects in the phantom that locally change the solution conductivity. Ideally, the object should contain enough water to produce a reasonable NMR signal, otherwise it would not appear in the NMR images. The polyacrylamide gel and the synthetic sponge tested here, perfectly achieve these aims. However, because the polyacrylamide gel made for this experiment only increased the impedance by $10 \%$ it was practically unnoticeable in the current images. In the sponge experiment the increased impedance is about $17 \%$ and the current image just starts to show the resultant changes in the current (figure 5.13).

In conclusion, this experiment demonstrated that because of the intrinsic NMR noise, it is very unlike that current changes produced by impedance variation of less than about $17 \%$ can be measured by the NMR technique. In chapter 6 the NMR noise is analysed and as shown in figure 6.4 the minimum current standard deviation in these phantom experiments is around $1 \mu \mathrm{A}$ which is more or less the same deviation indicated in the four central ROIs of figure 5.12 where the current is more uniform. (Since the higher gradient in the current density near to the electrodes affects the standard deviation, these values can not be considered for this analysis). 


\section{Chapter 6}

\section{Noise Analyses}

\section{Electrical current noise analyses}

The aim of this section is to review the NMR noise sources and determine the noise level in the electrical current measurement by the NMR technique. Since the electrical current image is computed from the NMR phase images the current standard deviation is related to the NMR noise. This section starts with a brief introduction to the main sources of noise in NMR, and some parameters related to the image acquisition that affects it. This is followed by the phase error propagation theory and finally the equation that determines the error in the current image is developed.

\subsection{Noise sources and SNR in NMR}

The sources of noise in NMR images can be grouped into two main types: those produced by the electronic system and those related to the subject or sample. The system noise sources are the electrical resistance in the receiver coil, pre-amplifier noise figure and RF transmitter noise. The subject noise sources are the dielectric and inductive losses (Hoult and Lauterbur, 1979; Hoult and Richards, 1976). The coil noise is the thermal, or Johnson, noise produced by the coil resistance $\left(R_{c}\right)$ that is proportional to $\sqrt{R_{c}}{ }^{14}$. The sample increases the noise due to the conductive losses in the tissues of the body, which lower the quality factor $Q$ of the receiving coil tuned circuit. It can be shown that the increase of noise due to the $Q$ factor is proportional to $\left(Q_{\text {empty }} / Q_{\text {full }}\right)^{1 / 2}$ (Libove and Singer, 1980). The dielectric sample losses are related to the coil distributed capacitance along its length. The electric field produced by the distributed capacitance across the sample dissipates energy orienting the molecular dipoles. These sources of noise are intrinsic to the NMR experiment and can only be reduced by careful

14 The rms value of the thermal-resistance, or Johnson, noise voltage $v_{n}$ over a frequency range $f_{H}-f_{L}$ is given by the expression (Millman and Halkias, 1972),

$$
v_{n}^{2}=4 k T R B
$$

where $\mathrm{k}$ is the Boltzmann constant, $J / F, T$ is the resistance temperature, ${ }^{\circ} K, R$ is the resistance, ohms, $B$ is the bandwidth, $f_{H}-f_{L}, H z$. The same noise power exists in a given bandwidth regardless of the centre frequency. Such a noise distribution is called white noise 
system and coil design, and also by signal averaging.

It can be shown that the noise standard deviation in the NMR image is inversely proportional to $\sqrt{N}$, where $\mathrm{N}$ is the number of averages of the NMR signal (McVeight, 1985 ), and directly proportional to the root square of the spectral resolution ${ }^{14}$, that is equal to $1 / \sqrt{T_{s}}$ where $T_{s}$ is the total sampling time use to sample the spin-echo signal. It is interesting to note that for the same sample time $T_{s}$, the number of sample points selected (which defines the number of pixels) does not affect the noise standard deviation (since it does not change the bandwidth of frequency within a pixel), but it does change the signal intensity and for larger pixels it improves the SNR as shown by equation (6.1).

These sources of noises in the NMR images are stochastic, with a Gaussian probability density function, stationary and ergodic (McVeight, 1985), see also section 6.5 about the noise in the NMR magnitude and phase images. By stochastic noise we mean that for each image (or a realisation of a process) the noise signal at a particular pixel is a random variable and can not be determined or avoided by any means. The ergodic condition implies that one image of a set of images (or ensemble) will have the same statistic as any other realisation of the ensemble. Finally, the noise in the NMR images is spatially invariant, or the statistic is the same over all regions in the image, which is called a stationary random process.

From the above considerations, the pixel SNR in the NMR image can then be determined by (Edelstein et al.,1986),

$$
S N R=\psi_{s} \Delta x \Delta y \Delta z f_{R} \sqrt{T_{s} N}
$$

where $N$ is the number of excitations used to average the spin-echo signal, $T_{s}$ is the total sampling time for a spin-echo signal, $f_{R}$ represents a factor related to relaxation times and proton density ${ }^{15}, \Delta x, \Delta y$ and $\Delta z$ are the pixel size, and $\psi_{s}$ is the SNR of the system plus the subject noise. $\quad \psi_{s}$ is measured with a cylinder

\footnotetext{
15 for an inverse recovery sequence $f_{R}$ is equal to,$$
f_{R}=\rho\left(1-e^{-\frac{T_{R}}{T_{1}}}\right) e^{-\frac{T E}{T_{2}}}
$$

where, $\rho$ is the proton density of the tissue of interest relative to the water, $T_{R}$ is the pulse repetition time, $T_{E}$ is the spin echo time, $T_{1}$ and $T_{2}$ are the longitudinal and transverse relaxation times respectively. 
phantom of volume $V$ in $m l$ and applying the following equation (Edelstein $e t$ al., 1984),

$$
\psi_{s}=\left[\frac{S}{\sigma}\right] \frac{K_{p}}{V}\left[\frac{1}{T_{S}}\right]^{1 / 2}\left[\frac{Q_{s}}{Q_{0}}\right]^{1 / 2}
$$

where $S$ and $\sigma$ are the signal averages and standard deviation along the NMR projection amplitude, $k_{p}$ is the number of points in the projection. The last term corrects the system SNR by considering the coil loading effect, where $Q_{0}$ is the quality factor for the coil alone and $Q_{s}$ is the quality factor for the coil with the subject inside.

In order to reduce the acquisition time it is possible to halve the number of phase encoding steps. For example, instead acquire a 256 consecutive spin-echoes it is possible to acquire only the 128 phase encoding steps in the centre region of the $k$-space and add 64 zeros to the ends of the $k$-space in the phase encoding direction. Therefore, for a time-domain matrix of 256 by 128, the final reconstructed image matrix after the zero filling and FT will have the same 256 by 256 pixels but would be acquired in half the time. The zero filling improves the noise standard deviation by $\sqrt{2}$ (McVeigh, 1985). On the other hand the zero filling increases the Gibbs phenomenon, which is evidenced as striations or edge ringing (Helkelman and Bronskill, 1987).

The decision about the pixel size is also a very important step in determining the final SNR of NMR images. From equation (6.1) it is possible to see that the SNR is directly proportional to the voxel size. As is shown in figure 6.1, unlike the case of ionizing radiation, where the SNR is proportional to the root square of the pixel area,

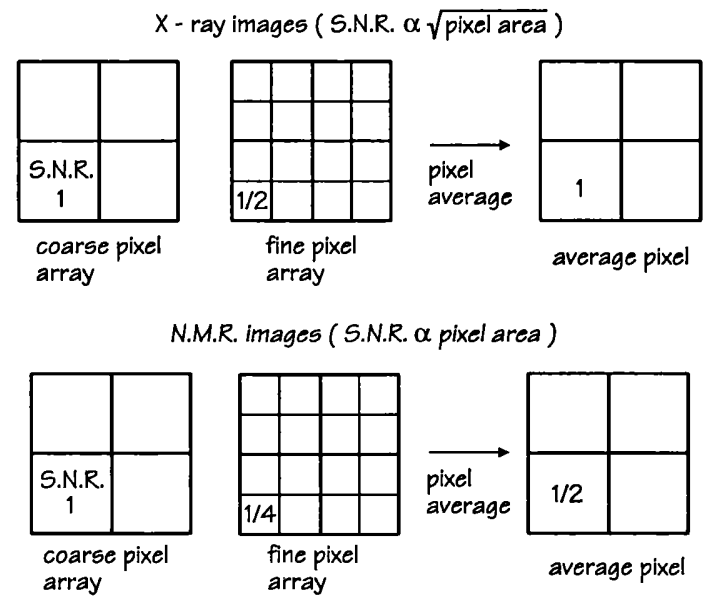

Fig. 6.1 - Effect of pixel size on SNR for ionizing radiation imaging and NMR imaging. For NMR images pixel averaging over a finer array recovers just half of that coarse array. 
in the NMR images, pixel averaging does not recover the SNR of a coarse array (McVeigh, 1985).

\subsection{Error propagation in NMR phase images}

As described in section 2.3 the NMR images are derived from two independent quadrature time-domain signals. These signals are Fourier transformed and phase corrected to produce the Absorption $A(\omega)$ and Dispersion $D(\omega)$ images. The NMR phase images are then calculated using equation (6.3).

$$
\varphi(\omega)=\tan ^{-1}\left(\frac{D(\omega)}{A(\omega)}\right)
$$

Ideally, the NMR signal for a perfectly uniform magnetic field and phase correction is concentrated in the absorption mode and the NMR phase image would be zero at any position over the object. As shown in figure 6.2 the NMR phase image has a very low SNR and the only reason that makes the phase image well defined in the field of view, FOV, is the noise background around it, which is caused by the signal (absorption and dispersion modes) fluctuation around zero. Therefore, differently from the magnitude image, it can not be referred to in terms of SNR but rather in terms of the phase standard deviation or phase error.

To determine the phase error accumulated from the absorption and dispersion modes, the theory of error propagation from two independent observables to two parameters is applied here (Cliffort, 1973). The variance in the phase image, $\sigma^{2}(\varphi)$, is determined by,
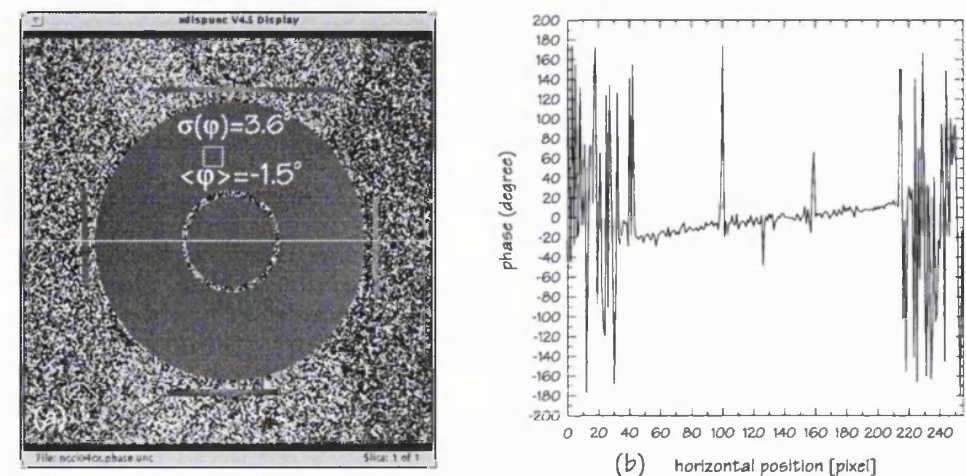

(b) horizontal position [pixel]

Figure 6.2 - The noise background in the NMR phase image (a) makes the object image well defined in the FOV, (b) horizontal image profile. 


$$
\sigma(\varphi)^{2}=E\left[(\varphi-\bar{\varphi})^{2}\right]
$$

where $\bar{\phi}$ is the mean or expected value $\boldsymbol{E}$ equals to,

$$
\bar{\varphi}=E[\varphi]=\sum_{j} \varphi_{j} P_{j}\left(\varphi_{j}\right)
$$

and $P_{j}\left(\varphi_{j}\right)$ is the probability of the event $\varphi_{j}$, equal to $P_{j}\left(\varphi_{j}\right)=\lim _{j \rightarrow \infty}\left(\frac{\varphi_{j}}{j}\right)$

The difference in equation (6.4) can be approximated by the Taylor's expansion function of two variables (Heading, 1970),

$$
\varphi-\bar{\varphi} \approx \frac{\partial \varphi}{\partial A}[A-\bar{A}]+\frac{\partial \varphi}{\partial D}[D-\bar{D}]
$$

substituting (6.6) into (6.4),

$$
\sigma(\varphi)^{2}=\boldsymbol{E}\left[\left(\frac{\partial \varphi}{\partial A}(A-\bar{A})+\frac{\partial \varphi}{\partial D}(D-\bar{D})\right)^{2}\right]
$$

and expanding the brackets squared results in,

$$
\begin{aligned}
\sigma(\varphi)^{2} & =\left(\frac{\partial \varphi}{\partial A}\right)^{2} \boldsymbol{E}\left[(A-\bar{A})^{2}\right]+\left(\frac{\partial \varphi}{\partial D}\right)^{2} \boldsymbol{E}\left[(D-\bar{D})^{2}\right] \\
& +2\left(\frac{\partial \varphi}{\partial A}\right)\left(\frac{\partial \varphi}{\partial D}\right) \boldsymbol{E}[(A-\bar{A})(D-\bar{D})]
\end{aligned}
$$

where $\boldsymbol{E}\left[(A-\bar{A})^{2}\right]$ is the noise variance of the absorption image, $\sigma^{2}(A)$, $\boldsymbol{E}\left[(D-D)^{2}\right]$ is the noise variance of the dispersion mode, $\sigma^{2}(D)$, and the last term contains the covariance between the absorption and dispersion modes, which can be written in terms of the correlation coefficient $\rho$ and standard deviation $\sigma$ as,

$$
\boldsymbol{E}[(A-\bar{A})(D-\bar{D})]=\operatorname{cov}(A, D)=\rho(A, D) \sigma(A) \sigma(D)
$$

Substituting equations (6.9) into (6.8), the variance in the phase image results,

$$
\begin{aligned}
\sigma(\varphi)^{2}=\left(\frac{\partial \varphi}{\partial A}\right)^{2} \sigma(D)^{2} & +\left(\frac{\partial \varphi}{\partial D}\right)^{2} \sigma(A)^{2}+ \\
& 2 \rho(A, D) \sigma(A) \sigma(D)\left(\frac{\partial \varphi}{\partial A}\right)\left(\frac{\partial \varphi}{\partial D}\right)
\end{aligned}
$$


Each partial derivative is determined using the chain rule ${ }^{16}$. Thus, for the phase partial derivative in relation to the absorption mode,

$$
\frac{\partial \varphi}{\partial A}=\frac{\partial \varphi}{\partial[\tan \varphi]} \frac{\partial[\tan \varphi]}{\partial A}
$$

where each partial derivatives is determined as follow,

$$
\begin{gathered}
\frac{\partial \varphi}{\partial[\tan \varphi]}=\frac{\partial\left[\tan ^{-1}\left(\frac{D}{A}\right)\right]}{\partial\left[\frac{D}{A}\right]}=\frac{1}{1+\left(\frac{D}{A}\right)^{2}}=\frac{A^{2}}{A^{2}+D^{2}}=\cos ^{2} \varphi \\
\frac{\partial[\tan \varphi]}{\partial A}=\frac{\partial\left(\frac{D}{A}\right)}{\partial A}=\frac{A\left(\frac{d D}{d A}\right)-D\left(\frac{d A}{d A}\right)}{A^{2}}=-\frac{D}{A^{2}}
\end{gathered}
$$

hence,

$$
\frac{\partial \varphi}{\partial A}=-\cos ^{2}(\varphi) \frac{D}{A^{2}}
$$

and for the phase partial derivative in relation to the dispersion mode,

where,

$$
\frac{\partial \varphi}{\partial D}=\frac{\partial \varphi}{\partial[\tan \varphi]} \frac{\partial[\tan \varphi]}{\partial D}
$$

$$
\frac{\partial[\tan \varphi]}{\partial D}=\frac{A\left(\frac{d D}{d D}\right)-D\left(\frac{d A}{d D}\right)}{A^{2}}=\frac{1}{A}
$$

hence substituting equations (6.12) and (6.15) into (6.14) gives,

$$
\frac{\partial \varphi}{\partial D}=\cos ^{2} \varphi \frac{1}{A}
$$

Finally, substituting equations (6.13) and (6.16) into (6.10), the variance in the phase image is,

$$
\sigma^{2}(\varphi)=\cos ^{4} \varphi \frac{D^{2}}{A^{4}} \sigma^{2}(A)+\frac{\cos ^{4} \varphi}{A^{2}} \sigma^{2}(D)-2 \rho \frac{\cos ^{4} \varphi}{A^{3}} D \sigma(A) \sigma(D)
$$

16 Chain rule $\frac{d y}{d x}=\frac{d y}{d u} \frac{d u}{d x}$ 
If the variances in the absorption and dispersion modes are assumed to be the same and equal to $\sigma_{0}$,

$$
\sigma^{2}(A)=\sigma^{2}(D)=\sigma_{0}^{2}
$$

and the correlation coefficient $\rho$ is equal to zero, equation (6.17) can be simplified to,

where

$$
\sigma^{2}(\varphi)=\sigma_{0}^{2}+\left[\frac{\cos ^{4}(\varphi)\left(A^{2}+D^{2}\right)}{A^{4}}\right]
$$

$$
|M|^{2}=A^{2}+D^{2} \text { and } \cos (\varphi)=\frac{A}{|M|}
$$

thus the standard deviation in the phase image is equal to,

$$
\sigma(\varphi)=\frac{\sigma_{0}}{|M|}
$$

This equation determines the phase standard deviation on a pixel by pixel basis (in radians) as the ratio between the signal standard deviation and pixel signal intensity inside of the object and was derived using the noise theory propagation by Conturo (1990). To measure the standard deviation in the magnitude image it is important to avoid pattern noise that may exist through the image. This can be done by selecting a small and uniform region to calculate the signal standard deviation. A related discussion about the measurements in the NMR magnitude images is presented in section 6.5 .

\subsubsection{Phase error propagation experiments}

A program was written in " $C$ " to solve the full equation (6.17) and the simplified equation (6.21) for any pixel position and region size within the FOV. The program read the real and imaginary images reconstructed with the routines described in section 2.3.5, calculates the standard deviation, variance and the correlation coefficient for each region selected, and solves the above equations on a pixel by pixel basis. To compare the results, it also computes the phase image and determines the phase standard deviation of the regions selected.

An NMR experiment was performed with the cylinder phantom described in section 4.1. The inner cylinder was filled with a solution of $0.2 \mathrm{mM} \mathrm{MnCl}$, with approximate $T_{1}=650 \mathrm{~ms}$ and $T_{2}=50 \mathrm{~ms}$ and the outer cylinder was filled with a solution 
of $0.058 \mathrm{mM} \mathrm{MnCl}$ plus $0.8 \mathrm{mM} \mathrm{CuSO}_{4}$, where the approximate $T_{l}=760 \mathrm{~ms}$ and $T_{2}=152 \mathrm{~ms}$. This range of values matches approximately the range of $T_{1}$ and $T_{2}$ values found in the newborn piglet brain in the first few days of life. Six time domain data sets of 256 spin-echos, each one sampled 512 times were acquired (FOV=12 cm, slice thickness $3 \mathrm{~mm}$, repetition time $1000 \mathrm{~ms}$ ). Each time domain set was acquired with a different echo-time $(50,100,150,200,300$ and $400 \mathrm{~ms})$ and reconstructed and phase corrected according to the routines described in section 2.3.5. After the FT, the 512 pixel columns was reduced to 256 by selecting the central square region of the FOV. The image pixel size is $0.47 \mathrm{~mm} \times 0.47 \mathrm{~mm}$. No electrical current was applied during the experiment. Tables 6.1 and 6.2 show the result obtained for the inner and outer cylinder respectively. The phase standard deviation values calculated and measured are given in degrees. Since the theoretical phase error is calculated on a pixel by pixel basis, the mean standard variation value ${ }^{17}$ of all the pixels within the selected region is also given in the tables. Figure 6.3 is a plot of the phase standard deviation predicted using equations (6.17) and (6.21), measured and the ratio $\langle M>/ \sigma(M)$ against the echo-time. Note in these plots the good agreement between the predicted and measured values whilst the ratio $\langle M>/ \sigma(M)$ is bigger than 2 .

\subsection{Error propagation in the current image}

As described in section 3.4 the current images are calculated by applying Ampere's Law in regions of 3x3 pixels in the NMR unwrapped phase images. Since the NMR experiment just measures the components of current magnetic field parallel to the main field $\boldsymbol{B}_{\boldsymbol{0}}$, the closed integral and the scalar product of Ampere's Law can be decomposed into simple integrals and simple products. The component of current $I_{y}$ is equal to (see figure 3.12 ),

$$
I_{y}=\oint \boldsymbol{H} \cdot d \boldsymbol{L}=\int_{1}^{3} H_{13} d L_{13}+\int_{3}^{5} H_{35} d L_{35}+\int_{5}^{7} H_{57} d L_{57}+\int_{7}^{1} H_{71} d L_{71}
$$

and applying the trapezoidal rule, the final equation for $I_{y}$ considering the standard

17 The mean standard deviation is determined by $\langle\sigma(\varphi)\rangle=\overline{\sigma(\varphi)}=\frac{1}{N}\left[\sum_{j}^{N}\left[\sigma_{j}(\varphi)\right]^{2}\right]^{1 / 2}$. 
Table 6.1 - Results for a region of 36 pixels within the inner cylinder. ${ }^{17}$

\begin{tabular}{||c|c|c|c|c|c|c|c|c||}
\hline $\begin{array}{c}T E \\
(m s)\end{array}$ & $\sigma(A)$ & $\sigma(D)$ & $\sigma(M)$ & $\begin{array}{c}M / \sigma(M \\
\langle\sigma(M) / M> \\
E q .(6.21)\end{array}$ & $\begin{array}{c}<\sigma(\varphi)> \\
E q .(6.17)\end{array}$ & $\begin{array}{c}\sigma(\varphi) \\
\text { measured }\end{array}$ & $\begin{array}{c}\rho \\
10^{-3}\end{array}$ \\
\hline \hline 50 & 16.54 & 14.10 & 13.71 & 14.54 & $3.97^{\circ}$ & $4.85^{\circ}$ & $4.88^{\circ}$ & 113.46 \\
\hline 100 & 13.50 & 15.32 & 14.91 & 6.32 & $9.45^{\circ}$ & $8.76^{\circ}$ & $8.71^{\circ}$ & 22.03 \\
\hline 150 & 14.38 & 14.64 & 13.97 & 3.58 & $18.69^{\circ}$ & $19.09^{\circ}$ & $19.82^{\circ}$ & -59.58 \\
\hline 200 & 13.94 & 14.25 & 12.08 & 2.28 & $56.20^{\circ}$ & $65.35^{\circ}$ & $78.11^{\circ}$ & 66.04 \\
\hline 300 & 14.82 & 14.78 & 9.26 & 2.15 & $66.38^{\circ}$ & $106.01^{\circ}$ & $108.23^{\circ}$ & -98.19 \\
\hline 400 & 13.62 & 13.76 & 8.56 & 2.05 & $50.54^{\circ}$ & $80.76^{\circ}$ & $105.56^{\circ}$ & -27.54 \\
\hline \hline
\end{tabular}

Table 6.2 - Results for a region of 36 pixels within the out cylinder ${ }^{17}$.

\begin{tabular}{||c|c|c|c|c|c|c|c|c||}
\hline $\begin{array}{c}T E \\
(m s)\end{array}$ & $\sigma(A)$ & $\sigma(D)$ & $\sigma(M)$ & $M / \sigma(M$ & $\begin{array}{c}<\sigma(M) / M> \\
E q .(6.21)\end{array}$ & $\begin{array}{c}<\sigma(\varphi)> \\
E q .(6.17)\end{array}$ & $\begin{array}{c}\sigma(\varphi) \\
\text { measured }\end{array}$ & $\begin{array}{c}\rho \\
10^{-3}\end{array}$ \\
\hline \hline 50 & 15.14 & 15.38 & 13.93 & 18.84 & $3.05^{\circ}$ & $3.61^{\circ}$ & $3.60^{\circ}$ & 167.91 \\
\hline 100 & 13.74 & 15.22 & 14.29 & 13.18 & $4.38^{\circ}$ & $4.51^{\circ}$ & $4.49^{\circ}$ & 80.43 \\
\hline 150 & 13.60 & 13.90 & 13.06 & 10.60 & $5.49^{\circ}$ & $6.02^{\circ}$ & $5.99^{\circ}$ & 102.42 \\
\hline 200 & 14.29 & 15.12 & 14.96 & 6.75 & $8.77^{\circ}$ & $8.48^{\circ}$ & $8.32^{\circ}$ & 6.98 \\
\hline 300 & 13.25 & 13.56 & 13.50 & 3.78 & $17.91^{\circ}$ & $19.85^{\circ}$ & $18.32^{\circ}$ & 16.70 \\
\hline 400 & 14.06 & 14.88 & 12.73 & 2.44 & $59.56^{\circ}$ & $68.48^{\circ}$ & $56.36^{\circ}$ & 97.69 \\
\hline \hline
\end{tabular}

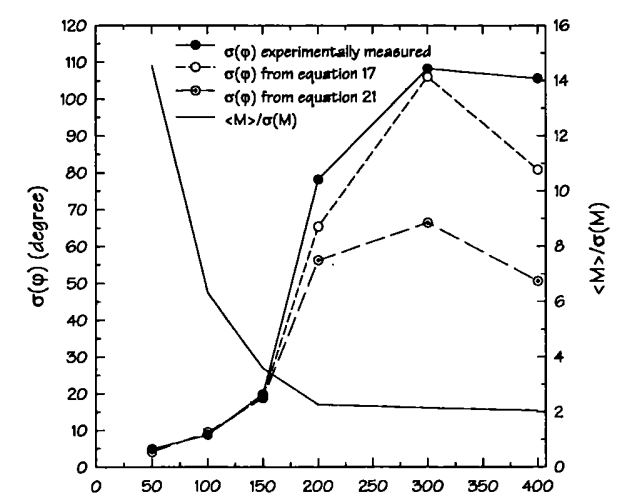

(a) echo-time (ms)

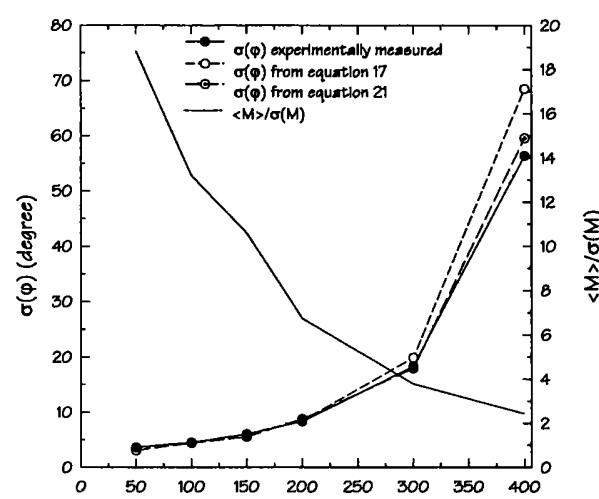

(b) echo-time ( $m s$ )

Figure 6.3 - Phase standard deviation and SNR vs echo-time. (a) inner cylinder (b) out cylinder. 
deviation $\sigma_{\boldsymbol{H}}$ of each measurement of the magnetic field $\boldsymbol{H}$ is,

$$
\begin{aligned}
& I_{y} \pm \sigma\left(I_{y}\right)=\frac{\Delta_{x}}{8}\left\{\left(H_{1} \pm \sigma_{H}\right)+2\left(H_{2} \pm \sigma_{H}\right)+\left(H_{3} \pm \sigma_{H}\right)\right. \\
&\left.-\left(H_{5} \pm \sigma_{H}\right)-2\left(H_{6} \pm \sigma_{H}\right)-\left(H_{7} \pm \sigma_{H}\right)\right\}+ \\
& \frac{\Delta_{z}}{8}\left\{\left(H_{3} \pm \sigma_{H}\right)+2\left(H_{4} \pm \sigma_{H}\right)+\left(H_{5} \pm \sigma_{H}\right)\right. \\
&\left.-\left(H_{7} \pm \sigma_{H}\right)-2\left(H_{8} \pm \sigma_{H}\right)-\left(H_{1} \pm \sigma_{H}\right)\right\}
\end{aligned}
$$

and $\boldsymbol{H}$ is determined from the NMR phase images by the following equation,

$$
H \pm \sigma_{H}=\frac{\varphi \pm \sigma(\varphi)}{\gamma T_{c} \mu_{0}}
$$

where $\gamma$ is the gyromagnetic constant, $T_{c}$ is the current time and $\mu_{0}$ is the permeability of free space. It is also assumed that all phase pixels values have the same standard deviation.

Substituting (6.24) into (6.23) and considering just the phase standard deviation results,

$$
\begin{aligned}
\sigma\left(I_{y}\right)= & \frac{\Delta_{x}}{8 \gamma T_{c} \mu_{0}}\left\{\sigma_{\varphi}+2 \sigma_{\varphi}+\sigma_{\varphi}+\sigma_{\varphi}+2 \sigma_{\varphi}+\sigma_{\varphi}\right\}+ \\
& \frac{\Delta_{z}}{8 \gamma T_{c} \mu_{0}}\left\{\sigma_{\varphi}+2 \sigma_{\varphi}+\sigma_{\varphi}+\sigma_{\varphi}+2 \sigma_{\varphi}+\sigma_{\varphi}\right\}
\end{aligned}
$$

and summing up the standard deviations ${ }^{18}$,

18

The sum of standard deviation. If $X=x(i, j)$ and $Y=y(i, j)$ are two independent random processes, and $Z=X+Y$ the variance in the sum is equal to,

$$
\begin{aligned}
\sigma^{2} & =\boldsymbol{E}\left[(z-\bar{z})^{2}\right]=\boldsymbol{E}\left[\left(z^{2}-2 z \bar{z}+\bar{z}^{2}\right)\right]=\bar{z}^{2}-\bar{z}^{2} \\
\bar{z}^{2} & =\boldsymbol{E}\left[(x+y)^{2}\right]=\boldsymbol{E}\left[x^{2}+2 x y+y^{2}\right]=\overline{x^{2}}+2 \bar{x} \bar{y}+\bar{y}^{2} \\
\bar{z}^{2} & =(\bar{x}+\bar{y})^{2}=\bar{x}^{2}+2 \bar{x} \bar{y} \bar{y}^{2} \\
\sigma_{z}^{2} & =\overline{x^{2}}+2 \bar{x} \bar{y}+\bar{y}^{2}-\bar{x}^{2}-2 \bar{x} \bar{y}-\bar{y}^{2}=\bar{x}^{2}-\bar{x}^{2}+\bar{y}^{2}-\bar{y}^{2} \\
\sigma_{z}^{2} & =\sigma_{x}^{2}+\sigma_{y}^{2}
\end{aligned}
$$

The same result is obtained if the two images are subtracted. Whenever when a subtraction or an summation is performed between two images the variance always will be the sum of each individual variances, increasing the noise in the final image. Thus the standard deviation is equal to,

$$
\sigma_{z}=\sqrt{\sigma_{x}^{2}+\sigma_{y}^{2}} \quad \text { and if } \sigma_{x}=\sigma_{y}=\sigma_{0} \quad \sigma_{z}=\sqrt{2} \sigma_{0}
$$




$$
\sigma\left(I_{y}\right)=\left(\frac{\sigma_{\varphi}}{4 \gamma T_{c} \mu_{0}}\right) \sqrt{3\left(\Delta_{x}^{2}+\Delta_{z}^{2}\right)}
$$

and applying equation (6.21) into (6.26), the final current image standard deviation is equal to,

$$
\sigma\left(I_{y}\right)=\left(\frac{1}{4 \gamma T_{c} \mu_{0}}\right) \frac{\sigma(|M|)}{|M|} \sqrt{3\left(\Delta_{x}^{2}+\Delta_{z}^{2}\right)}
$$

To eliminate any starting phase shift produced by the NMR system, field imperfections or remaining phase after the zero and first order correction, the images acquired with the electrical current and with no current are subtracted. The result is just the phase shift produce by the electrical current magnetic field. The noise in the subtracted image increases by $\sqrt{2}$,

$$
\sigma\left(I_{y}\right)=\left(\frac{1}{4 \gamma T_{c} \mu_{0}}\right) \sqrt{2} \frac{\sigma(|M|)}{|M|} \sqrt{3\left(\Delta_{x}^{2}+\Delta_{z}^{2}\right)}
$$

\subsubsection{Current image error propagation experiment}

The same NMR experiment set up described in section 6.2.1 was used to acquire three sets of images to calculate the electrical current in the $y$-direction. Each set was acquired with different echo-times (50,100, 150, 200, 300 and $400 \mathrm{~ms})$. Since the aim is to verify the error propagation, no current was applied during the NMR experiment. This procedure avoids the problem of image registration that may add error in the final current images. The current images were calculated as described in section 3.4. Table 6.3 gives the noise predicted using equation (6.28) and measured in the current image.

Figure 6.4(a) and (b) shows a plot of the current standard deviation measured, predicted with equation (6.28) and the ratio $\langle M>/ \sigma(M)$ versus echo-time. The $\sigma(|M|)$ used for the predicted plots were the mean standard deviation values show in tables 6.1 and 6.2. The signal intensity $|M|$ was determined by fitting an exponential decay equation to the NMR signal intensity measured for the six different echo-times mentioned above. The plot shows that the predicted and measured values of $\sigma\left(I_{y}\right)$ have a good agreement as long as the $\langle M>/ \sigma(M)$ is greater than 3 . 
Table 6.3 - Current noise predicted with equation (6.28) and measured.

\begin{tabular}{||c||c|c|c||c|c|c||}
\hline \multirow{2}{*}{\begin{tabular}{c}
\multirow{2}{*}{$\begin{array}{c}T E \\
(m s)\end{array}$} \\
\cline { 2 - 7 }
\end{tabular}} & \multicolumn{3}{c||}{ inner cylinder } & \multicolumn{3}{c||}{ outer cylinder } \\
\cline { 2 - 7 } & measured & predicted & $M / \sigma(M)$ & measured & predicted & $M / \sigma(M)$ \\
\hline 50 & 1.81 & 1.67 & 14.54 & 1.25 & 1.29 & 18.84 \\
\hline 100 & 2.11 & 1.99 & 6.32 & 0.983 & 0.923 & 13.19 \\
\hline 150 & 3.38 & 2.62 & 3.59 & 0.915 & 0.770 & 10.60 \\
\hline 200 & 7.44 & 5.92 & 2.27 & 0.909 & 0.924 & 6.75 \\
\hline 300 & 7.64 & 4.66 & 2.15 & 1.28 & 1.26 & 3.78 \\
\hline 400 & 5.71 & 2.66 & 2.05 & 3.09 & 3.14 & 2.44 \\
\hline
\end{tabular}

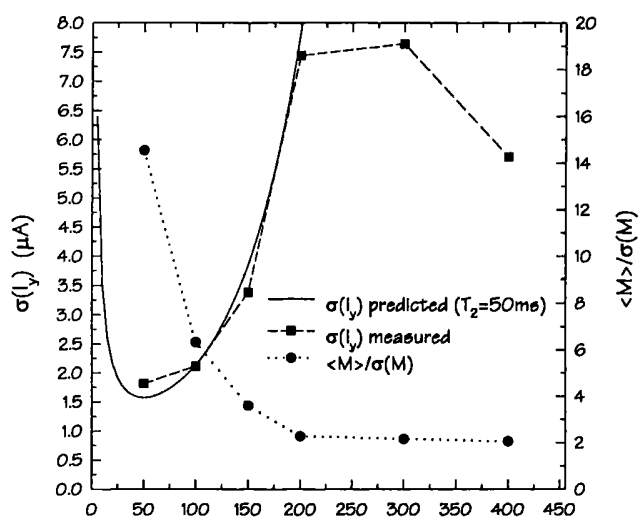

(a) echo-time (ms)

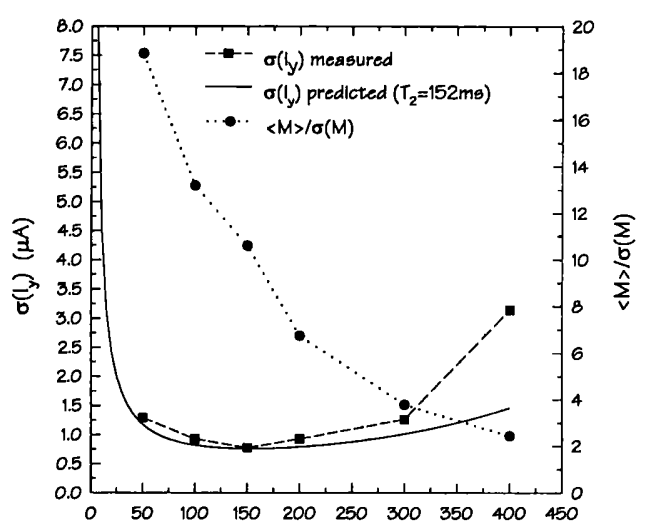

(b) echo-time (ms)

Figure 6.4 - Current standard deviation and $\langle M>/ \sigma(M)$ vs echo-time.

\subsection{Mis-registration error propagation}

The aim of this sub-section is to provide a figure for how much the current image is affected by any image mis-registration. The error introduced in the final current image by mis-registering the NMR images will obviously depend on the object symmetry and mainly on the current distribution, but even so, for reasonably uniform current distribution and for small differences in the image registration the results should be more or less the same.

This study was done using the data from the cube phantom experiments described in chapter 5 . The reason for using this data is that the platform it contains with the two holes of different sizes makes the current distribution inside of the cube 
very non-uniform and more similar to the situation expected in vivo. Also, if the ROIs used to integrate the current were chosen in the centre of the image, the border artifacts or any other structure that might exist in the image could be easily avoided. Since only the component of current orthogonal to the images can be quantified, just the effect on the $I_{x}$ component was studied.

The field images with the $y$-axis parallel to $\boldsymbol{B}_{\boldsymbol{0}}$, (figure 5.3(e)), were used as the reference and the images with the $z$-axis parallel to $\boldsymbol{B}_{\boldsymbol{0}}$, (figure 5.3(h)), were moved up and down and to the left and to the right by a known number of pixels. Each time the $z$-images were moved the current images were re-calculated and the current at three selected ROIs of 17 by 17 pixels was measured. Two ROIs were placed near to the holes in the platform and one in the centre of the cube image. In each direction, the $z$ images were moved 14 pixels and the error was calculated assuming the current through each region at the starting position as the initial value. Figure 6.5 shows a plot of the error versus pixel shift.

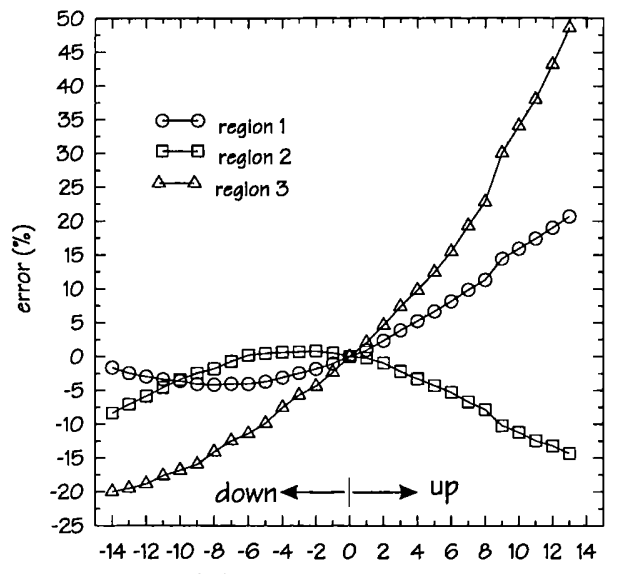

(a) shift [pixel]

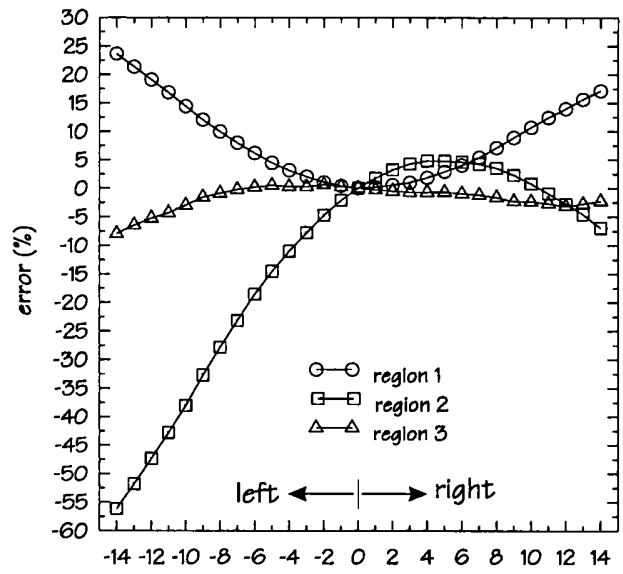

(b) shift [pixel]

Figure 6.5- The error caused by miss-registering the images during the image processing. (a) shift up and down, (b) shift left and right.

\subsection{The difference when SNR is lower than two}

In figure 6.3 it was show that the standard deviation of the measured phase and that calculated using equation (6.21) do not agree when the SNR is lower than three. In a very recent review about NMR noise theory (Gudbjartsson et al., 1995) the reasons for this difference were made clear. This theory was not applied first because the NMR image SNR was bigger than six, which makes the procedure that was applied here 
perfectly correct and secondly because the programs were implemented before this publication. Nevertheless, considering the aim of this chapter, we decided to extend the noise theory discussion when the SNR is lower than two. The following review is based upon the Gudjartsson (1995) publication.

In NMR the noise in both quadrature signals in the time-domain has a Gaussian distribution as explained in section 6.1. The absorption, $A$, and dispersion, $D$, modes are reconstructed through the complex FT, which is a linear and orthogonal process and it will preserve the Gaussian characteristic of the noise. The magnitude image are however calculated pixel by pixel by $M=\left[A^{2}+D^{2}\right]^{1 / 2}$, and this is not a linear step and the noise distribution in the magnitude image is therefore no longer Gaussian. In fact it has been shown that the noise distribution in the magnitude image will follow the Rice density given by (Carlson, 1975),

$$
P_{M}(M)=\frac{M}{\sigma^{2}} e^{-\left(S^{2}+M^{2}\right) /\left(2 \sigma^{2}\right)} I_{0}\left(\frac{M S}{\sigma^{2}}\right)
$$

where $M$ is the signal intensity measured in the magnitude image, $S$ is the signal intensity in the absence of noise, $\sigma$ is, as defined before, the Gaussian standard deviation in the absorption and dispersion modes and $I_{0}$ is the modified zeroth order Bessel function of the first kind ${ }^{19}$. Equation (6.29) can be simplified under larger-signal conditions to ${ }^{19}$,

$$
p_{M}(M) \approx \frac{1}{\sqrt{2 \pi \sigma^{2}}} e^{-\left(M-\left[S^{2}+\sigma^{2}\right]^{1 / 2}\right)^{2} /\left(2 \sigma^{2}\right)}
$$

since the exponential term in this equation dominates, the envelope PDF is essentially a gaussian curve with variance $\sigma^{2}$ and centred at $\left[S^{2}+\sigma^{2}\right]^{1 / 2}$. This is a small deviation for large SNR, however, when the SNR is small the measured value $\langle M\rangle$ is not the true expected signal intensity $S$, but an approximation. Figure 6.6 illustrates the transition of

$$
I_{0}(v)=\frac{1}{2 \pi} \int_{-\pi}^{\pi} e^{v \cos \phi} d \phi \text { with the properties } I_{0}(v) \approx \begin{cases}e^{v^{2 / 4}} & v<<1 \\ \frac{e^{v}}{\sqrt{2 \pi v}} & v>>1\end{cases}
$$


the envelope PDF from a Rician curve to a Gaussian curve as $S$ become large compared to $\sigma$, and the corresponding mean value of each curve is indicated by the vertical line. A look up table (Henkelman, 1986) and an analytical equation (Gudbjartsson and Patz, 1995) to correct the difference between the measured value $\langle M\rangle$ and the true expected value $S$ can be found in the literature.

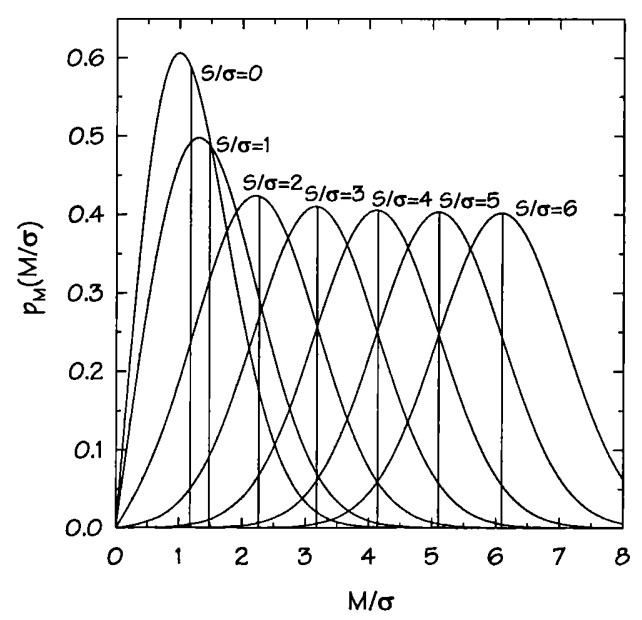

Figure 6.6 - The Rician distribution for several SNR and the corresponding mean.

Since the NMR phase image is calculated through the arctangent function its noise distribution is also no longer Gaussian, but it can be approximate to it if the SNR is greater than three. In fact, it can be shown through a completely different derivation (Carson, 1975) to that applied in this chapter that when $S>>\sigma$ the deviation in the phase angle, $\Delta \theta$, due to the noise has a distribution equal to,

$$
p_{\Delta \theta}(\Delta \theta) \approx \frac{1}{\sqrt{2 \pi(\sigma / M)^{2}}} e^{\left(\frac{-\Delta \theta^{2}}{2(\sigma / M)^{2}}\right)}
$$

In this equation the phase standard deviation is equal to that in equation (6.21) with a zero mean. To apply this equation the values of $M$ and $\sigma$ were measured at small ROIs in the intensity image. Hence, when the SNR is small, as shown in figure 6.6, the mean value starts to deviate from the true signal $S$ and errors in the calculated standard deviation of the phase are introduced.

Although the noise study described here does not affect at all the results of the noise analyses performed in this thesis, because in all the experiments the SNR is greater than six, it does explain the difference between the measured and calculated 
values when the SNR is lower then three.

\subsection{Discussion}

The NMR image noise has been analysed and some image acquisition parameters that affects the SNR, such as signal average, pixel size, zero filling were introduced. If just the noise is considered, the only way to reduce it without increasing the time of image acquisition is by increasing the spectral resolution, which means a longer sampling time, $T_{s}$, for each spin-echo. The increase in the $T_{s}$ also reduces the maximum frequency and requires the cutoff frequency of the low pass to be filter adjusted.

The signal maybe increased if the number of sample points and phase encoding steps are reduced to half, quarter, etc, keeping the same FOV size. This will increase the pixel size and reduce the time of image acquisition, but the resolution will be lower.

From tables 6.1 and 6.2 the standard deviation in the absorption and dispersion modes have approximately the same values, and the correlation coefficient is approximately equal to zero. Which means that both quadrature NMR signals have the same uncorrelated noise.

The current error propagation theory shows a good fit with the values measured in the current image experiments, as long as the ratio between the signal average and standard deviation is greater than two. The reason for this is that the true noise distribution in the NMR magnitude image is a Rician, but it approximates to Gaussian when large signals are present. In the graph plotted in figure 6.4, for the last two measurements in the inner cylinder $\left(T_{2}=50 \mathrm{~ms}\right)$ for $T E=300$ and $400 \mathrm{~ms}$, the ratio is near 2 and these points do not match the theoretical result. Actually for these lower ratio values it is almost impossible to distinguish the image from the background noise.

The current standard deviation also increases very sharply for small current integration times as shown in figure 6.4, due to the short magnetic current-spin integration period. For the worse case $T_{2}=50 \mathrm{~ms}$ (figure $6.4(\mathrm{a})$ ), the best applied currenttime would be around 50 to $100 \mathrm{~ms}$ which would give a current standard deviation around $2 \mu \mathrm{A}$. For longer $T_{2}$ (figure 6.4(b)), the current standard deviation will be smaller at, approximately $1 \mu \mathrm{A}$. Other points that may affect the current image error include: the image registration and the precision with which the $90^{\circ}$ rotation and the slice position selection is performed during the image acquisition.

Equation (6.27) is similar to the equation given by Scott et al. (1992b), the 
difference being in the SNR measurement where, they used the approach given Edelstein et al. (1986). We found easier to determine the noise in the current image using the Conturo et al. (1990) approach to determine the phase error.

The mis-registration study showed that the error in the current image is very dependent on the direction of the shift and that for this particular cube experiment, a shift of just 4 pixels, which is equivalent to approximately $2.5 \mathrm{~mm}$, can result in a error in the integrated current of about $10 \%$.

Finally, since in the previous experiments no current was applied we have to consider if any noise is introduced by the current source during the NMR experiment. This question can be answered by comparing the noise standard deviation in the simulation experiment (Chapter 5). There the current time is equal to $60 \mathrm{~ms}$ and $T_{2}$ equal to $800 \mathrm{~ms}$ and the noise level is around $0.8 \mu \mathrm{A}$ (table 4.1 ), which is within the values show in table 6.3 for the echo-times between 100 and $150 \mathrm{~ms}$. Therefore we conclude that no significant noise is introduced by the current source.

\subsection{Conclusion}

Two main types of error exist in the current images, the noise standard deviation due to the NMR noise and the error introduced by image mis-registration, the latter ever being dominant over the former. The image registration process will never be perfect and error will always be introduced in the current images. From the experiment performed in this thesis we can probably expected a mis-registration of at least 4 pixels which means that the current integrated error will have a minimum error of $10 \%$. 


\section{Chapter 7}

\section{Results: In vitro Current Images in the Piglet Head}

\section{In vitro experiments with new born piglet}

The aim of the in vitro experiments with the new born piglet was to make a preliminary study of the electrical current distribution in the head and to establish a methodology to be used during the in vivo experiment. A total of six in vitro experiments were performed, of which only the last three are presented here. The first three attempts did not work well due to problems with the stereotactic frame constructed to hold the piglet's head in the centre of the NMR magnet. The experiments presented here are divided into two parts, the two experiments performed with isolated heads and an experiment performed with an intact piglet.

The initial experiments were performed with an isolated head because the $2.4 \mathrm{~T}$ NMR machine at UCL has a small bore of $40 \mathrm{~cm}$. The image coil bore of $21 \mathrm{~cm}$ makes it physically impossible to rotate the whole piglet's body by $90^{\circ}$. For the in vitro experiment with the intact animal, the head was rotated by just $45^{\circ}$ in relation to the main NMR field.

This chapter starts by describing the isolated head experiments, the methodology used and the results. Next the experiment performed with the intact piglet is described. The noise in the current images is then analysed and the chapter ends with a brief discussion and conclusion.

\subsection{In vitro experiments with isolated head}

\subsubsection{Methodology}

The piglets used in these experiments were one day old sacrificed piglets that had been used as part of a separate study into hypoxic-ischaemic brain injury. The piglets had usually been killed by $\mathrm{N}_{2}$ inhalation or anaesthetic overdose, which should not affect the results presented here. The experiments were performed between six and seven hours after death and during this period the piglet was stored in a refrigerator at $4^{\circ} \mathrm{C}$. The head was then removed by severing the neck and placed in a special frame constructed to hold it accurately in the centre of the NMR magnet. The subsequent studies took 
about four hours to complete.

\subsubsection{The piglet's head holder and image markers}

The frame to hold the piglet's head was made of perspex with a cubic structure to help with the $90^{\circ}$ rotation and is illustrated in figure 7.1. To hold and position the head in the centre of the frame, three adjustable locating points were used, two on the side of the head and one in the mouth. The side gripping points are made of "Macor" ceramic, and are pressed into the skull by nylon screws which can move in slots on the sides of the frame. The mouth holder consists of two bars also made of perspex, one goes inside of the mouth and the other above it. These are pressed against each other by adjustable screws on threaded plastic rods thus gripping the top of the jaw. To help with image registration during the image processing, the four corner columns of the
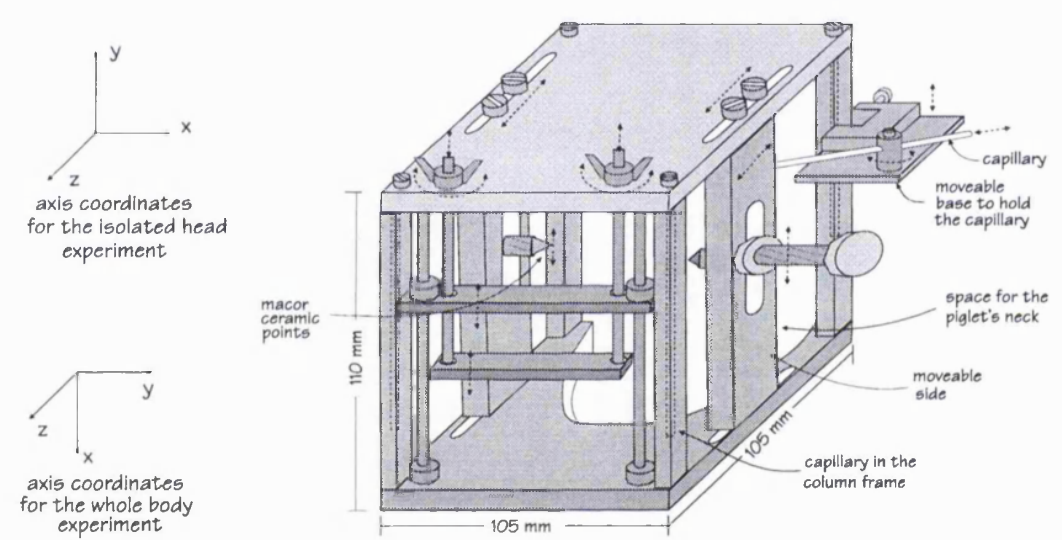

Figure 7.1- Frame to hold the piglet's head inside the magnet during the NMR experiment.

frame have a hole of $2 \mathrm{~mm}$ diameter drilled along their centres. The holes were filled with $\mathrm{CuSO}_{4}$ solution and sealed with nylon screws. These fluid filled tubes appear in the images as white dots.

During the NMR experiment the head needs to be rotated. For the first two positions ( $x$-and $z$-axis parallel to $\boldsymbol{B}_{0}$ ) the NMR tomographic plane is selected as coronal, and there is no problem of mis-registration of the slice position during the data acquisition. However, for the third image the head is placed on its side and the NMR tomographic plane needs to be changed from the coronal to a transverse slice orientation. This requires reference points in the image in order to match the transverse slice with the previous coronal view. Three methods were tried to help to physically register the slice position. First we tried to use a triangular shaped section filled with 
distilled water doped with $\mathrm{CuSO}_{4}$ to reduce the relaxation times. The triangle was placed at the side of the head and held in the columns of the frame. By measuring the length of the water column in the image, it should have been possible in principle, to determine the slice position after each rotation. Unfortunately, this technique did not work very well since the length of the column of water that appeared in the image along the side of the triangle hypotenuse was difficult to measure with sufficient precision. We also tried to place an inclined capillary between the columns of the frame. If the distance between the capillary in the column of the frame and the inclined capillary is equal to the measurement made before the rotation, then the slices are at same position. The problem with the inclined capillary is that the shim artifacts may move the capillaries' position in the image and the slice maybe set out of position. We tried this procedure in the second experiment described here. Finally, it was found to be much easier to place two capillaries near the head but rigidly fixed in the frame and from the plane of the capillaries, define the slice position. Figure 7.1 illustrates one of the two perspex structures used to hold the capillaries on the right side of the frame. This allows the capillaries to be moved up and down, forward and backward with respect to the head and rotated. Therefore, after each $90^{\circ}$ head rotation a fast image scan was performed to localise the capillaries and from this to define the correct tomographic plane.

\subsubsection{The electrodes and the electrical current pulses}

The electrodes used were infant carbon ECG electrodes (X-ray translucent Arbo H87VT) to minimise distortion of the main magnetic field. In both experiments the electrodes were placed on the top of the head and under the neck which corresponds to the $y$-direction of the current components. The reasons for placing the electrodes in this way are explained in the discussion section. To reduce the electrical contact resistance the regions under the electrodes were shaved.

In the first experiment, the total applied current time was set to $60 \mathrm{~ms}(30 \mathrm{~ms}$ for each pulse of current) with amplitude of $48.8 \mathrm{~mA}$ and a cycle repetition of $1000 \mathrm{~ms}$. In the second experiment, the total current time was set to $120 \mathrm{~ms}(60 \mathrm{~ms}$ for each pulse of current) with amplitude of $24.4 \mathrm{~mA}$ and repeated every $1200 \mathrm{~ms}$. In both cases the current is turned off for $4 \mathrm{~ms}$ during the $180^{\circ}$ refocusing pulse.

In each experiment, NMR data was collected for a positive current applied in the $y$-direction (positive-negative current sequence - PN) and another with a negative current 
in the -y-direction (negative-positive current sequence - NP). We found that the contrast between the final current images helped to define the electrical current distribution.

\subsubsection{The NMR image parameters}

The spin-echo pulse sequence (Multi-Slice Variable Echo - MSVE) used for the first experiment was set to a repetition time (TR) of $1000 \mathrm{~ms}$, echo time $60 \mathrm{~ms}$, slice thickness $3 \mathrm{~mm}$, field of view $18 \mathrm{~cm}$. The isocentre distances were set to $26 \mathrm{~mm}$ for the top slice, $23 \mathrm{~mm}$ for the centre slice and $20 \mathrm{~mm}$ for the bottom slice, giving a sequence of three adjacent slices. 256 by 256 image matrices with pixels of 0.70 by $0.70 \mathrm{~mm}$ were used in both transverse and coronal slices. The total scan time for each image was 4.34 minutes.

For the second experiment the repetition time was $1200 \mathrm{~ms}$, echo-time $120 \mathrm{~ms}$, slice thickness $3 \mathrm{~mm}$ and FOV $15 \mathrm{~cm}$. In this experiment just one transverse slice for each head orientation was acquired, and the isocentre was equal to $26.11 \mathrm{~mm}$. The image matrix was 256 by 256 pixels of $0.59 \mathrm{~mm}$ by $0.59 \mathrm{~mm}$. The total scan time for each image in these experiment was 5.20 minutes.

\subsubsection{Results for the first experiment}

Figure 7.2 shows the NMR magnitude images for the first in vitro piglet experiment with an isolated head. The images are divided into three sets (or columns in the figure), each set corresponds to one head orientation in relation to the main magnet field $\boldsymbol{B}_{\boldsymbol{0}}$ and each set is composed of three different slice positions called bottom, centre and top slices (rows in the figure). All tomographic planes shown are parallel to the $x z$-plane. The piglet's head was placed upright and the $z$-axis was defined along the head with the frame position indicated in figure 7.1. The images with the $x$-and $z$-axes parallel to $\boldsymbol{B}_{\boldsymbol{0}}$ shown in figure 7.2 (left and right columns) are coronal slices. After the $90^{\circ}$ rotation performed to bring the $y$-axis parallel to $\boldsymbol{B}_{\boldsymbol{\theta}}$, the slice orientation was set to transversal (centre column in figure 7.2). The centre slice matches the two glass capillaries ( $2 \mathrm{~mm}$ inner diameter) positioned at the level of the eyes. The capillaries were filled with $\mathrm{CuSO}_{4}$ solution $\left(T_{1}\right.$ and $T_{2}$ approximately $\left.100 \mathrm{~ms}\right)$. The distortions in the images of the capillaries shown in figure 7.2 centre row are due to shim artifact because of the distance away from the centre of the head. 


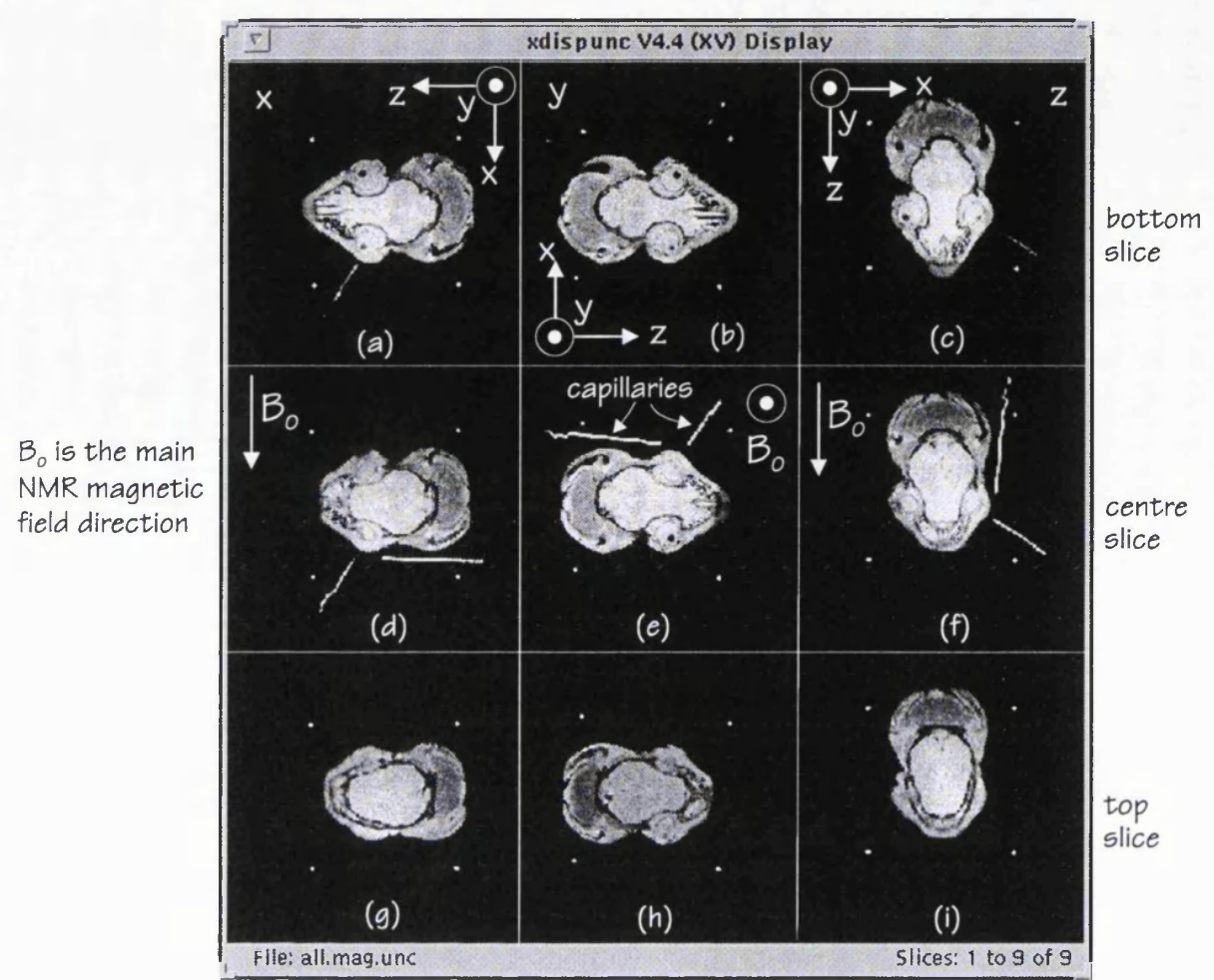

Figure 7.2 - No current NMR magnitude images of the top, centre and bottom slices for the three orientations $x, y$ and $z$ of the head in relation to the main magnetic field.

\subsubsection{Electrical current magnetic field}

The time domain data once acquired in the NMR system were transferred to the network, Fourier transformed and phase corrected as described in section 2.3.5. Next the phase images were calculated, phase unwrapped, registered, and scaled to determine the electrical current magnetic field (section 3.4). Figure 7.3(a) shows the magnetic field produced by the positive current and $7.3(\mathrm{~b})$ by the negative current at the centre slice position.

The black regions represent lower field, and the white regions higher field, values for the $B_{x}$ and $B_{z}$ field components and can be seen qualitatively to agree with the "right hand rule" predictions for the magnetic field orientation with respect to the current direction. For the $B_{y}$ component it is necessary to visualize how the electrical current propagates within the tomographic plane.

A vector plot of the magnetic field is shown in figure 7.4(a) for the positive current and figure 7.4(b) for the negative current. The vector plot was made using the $B_{x}$ and $B_{z}$ images shown in figure 7.3. The number of image pixels was reduced by using a 3 by 3 mask to average the signal intensity. A program was implemented in $\mathrm{C}$ 

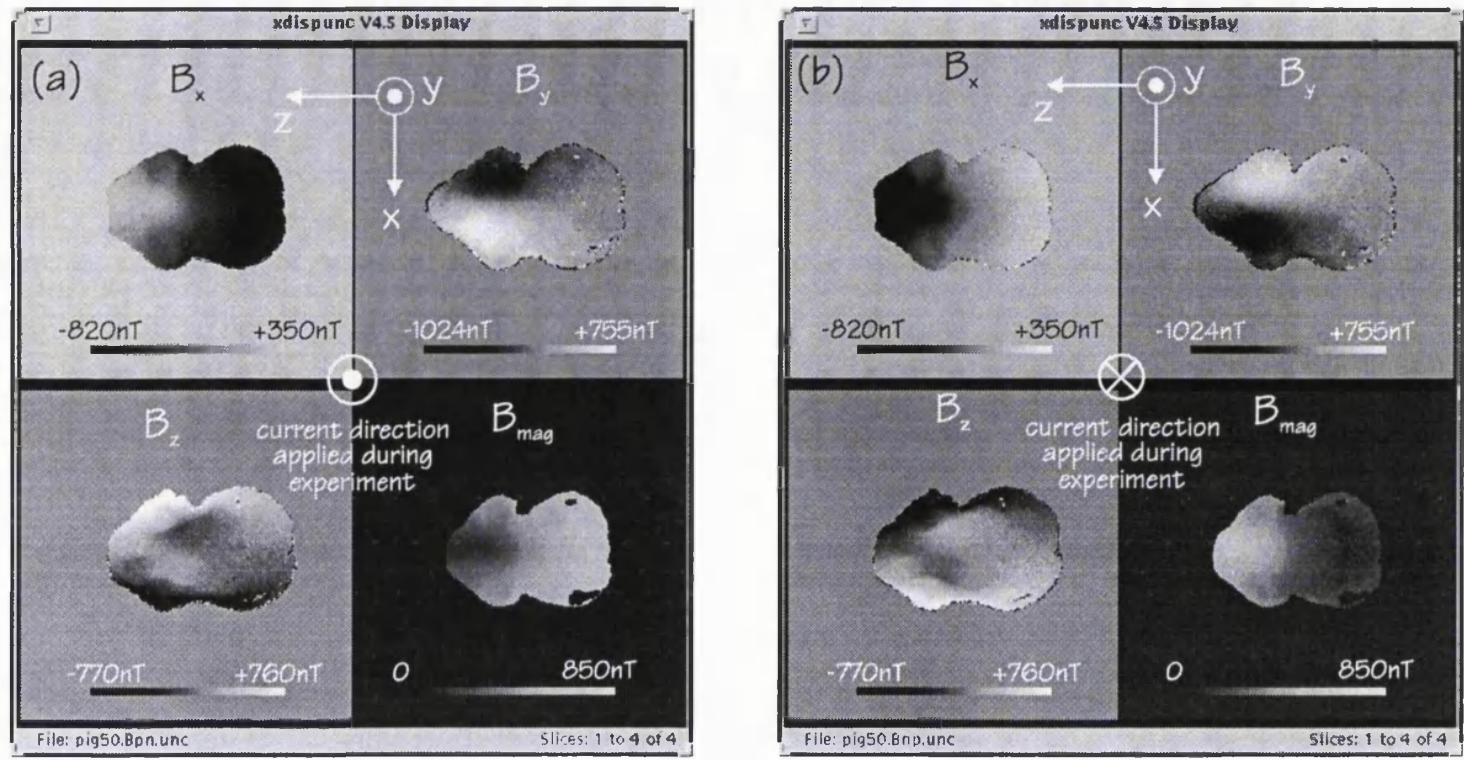

Figure 7.3-Images of the current magnetic field components $B_{x}, B_{v}, B_{z}$ and $B_{\text {mag }}(a)$ for the positive current (b) for the negative current.

to perform this task and also to store the averaged data in a format suitable for "Mathematica" which was used to plot the vector images. Using the right hand rule it is possible to check the field direction.

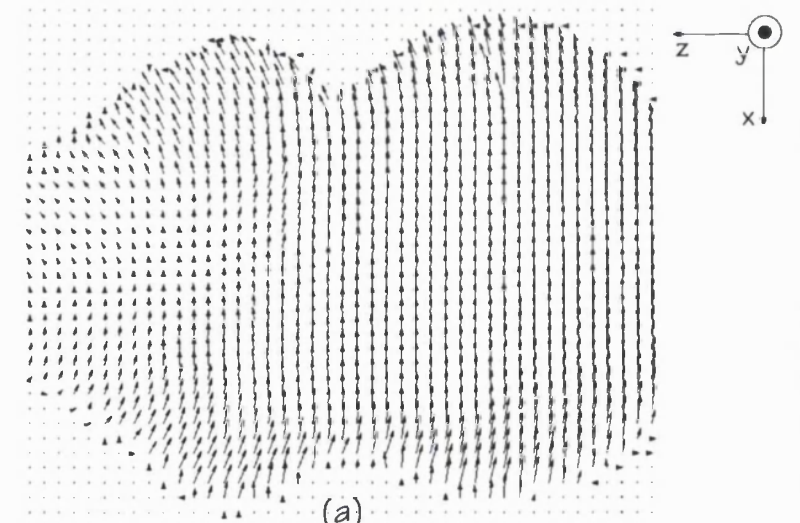

(a)

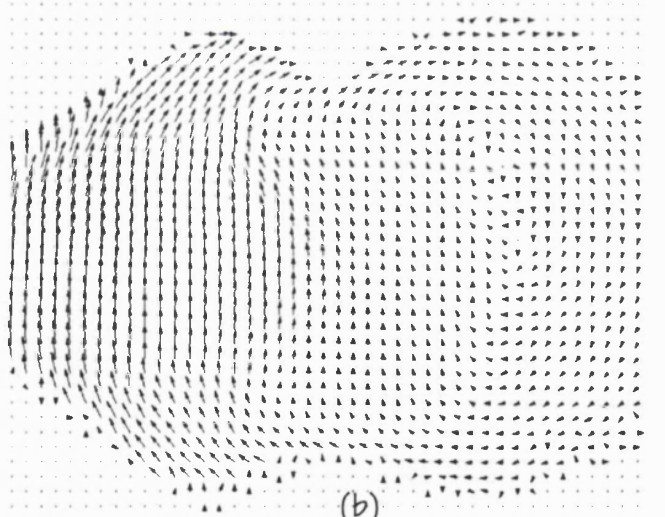

(b)

Figure 7.4-Current magnetic field vector plot using figure $7.3\left(B_{x}\right.$ and $\left.B_{z}\right) .(a)$ positive current $(b)$ negative current.

\subsubsection{Electrical current images}

Figure 7.5 shows the images of the electrical current component in the $x$ direction of the tomographic plane $\left(I_{x}\right)$. Figure $7.5(\mathrm{a})$ is the case where a positive electrical current was applied along the $y$-axis. Figure $7.5(\mathrm{~b})$ is the case for a negative current. The contrast between these images helps to define the places where the current is propagating, since brighter regions in (a) are darker in (b) and vice-versa. 

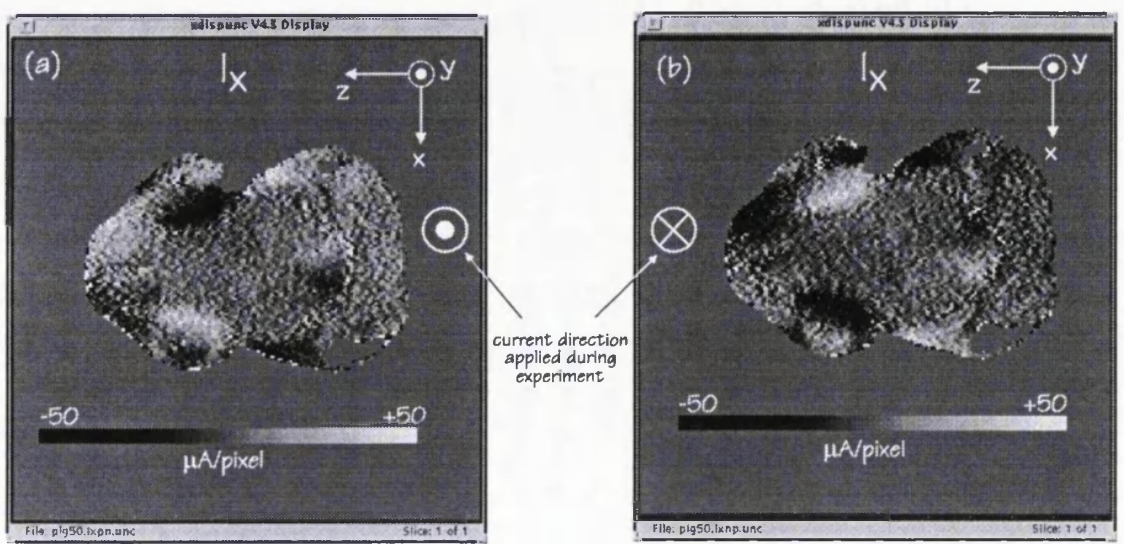

Figure 7.5 - $I_{x}$ current component, (a) for the positive current and $(b)$ for the negative current.

Figure 7.6 shows the images of the electrical current component in the $y$ direction $\left(I_{y}\right)$ for three adjacent slices. Figure 7.6(a) and (d) are the top slice, figure 7.6(b) and (e) are the centre slice and figure 7.6(c) and (f) are the bottom slice, respectively for the positive current and for the negative current. To help to decide where the current is concentrated all the images in figure 7.6 have the same range of values, from $-45 \mu \mathrm{A}$ to $+30 \mu \mathrm{A}$ per pixel (or from $-91 \mu \mathrm{A} / \mathrm{mm}^{2}$ to $60 \mu \mathrm{A} / \mathrm{mm}^{2}$ ). These figures show that most of the electrical current goes around the outside of the head and
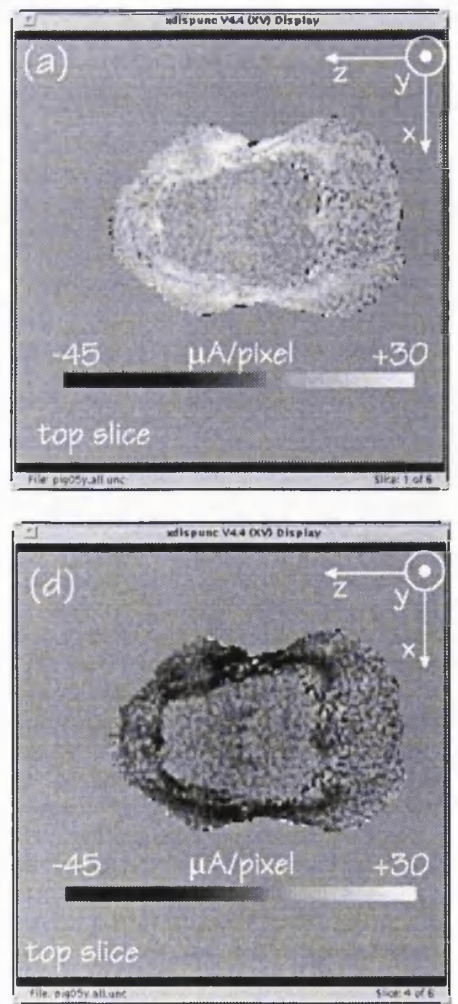
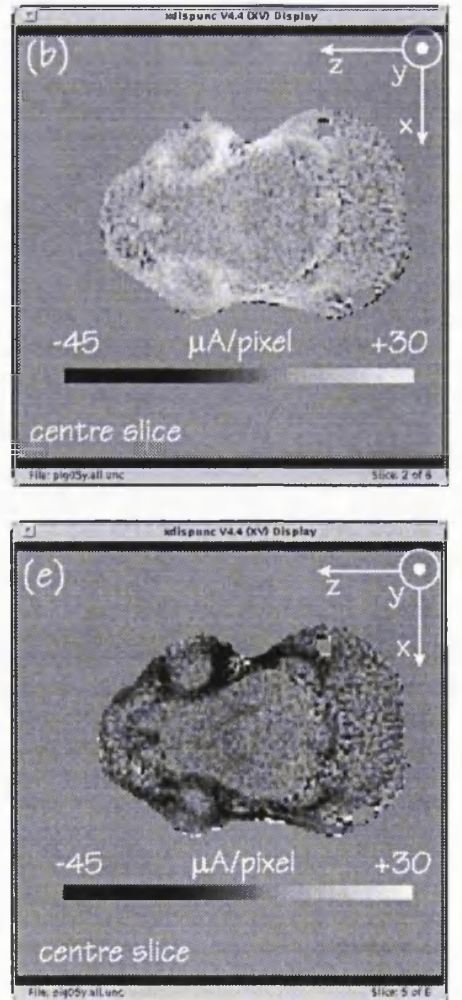
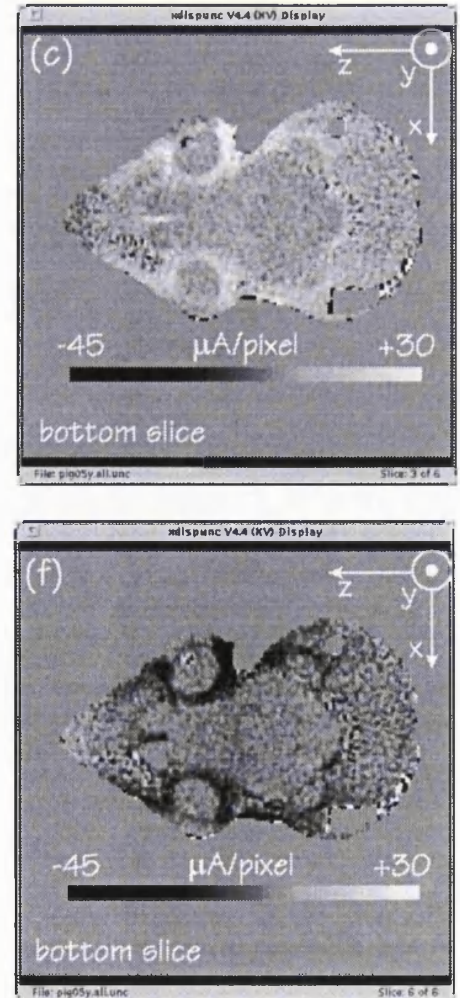

Figure 7.6- $I_{v}$ current component images in the top, centre and bottom slices. First row for the current in the positive $y$-direction and the other for the current in the negative $y$-direction. 
not through the brain.

Figures 7.7(a) and (b) show the current components in the $z$-direction. As with the $I_{x}$ data, the $I_{z}$ component, is also split into a positive and negative applied current direction. The dark region at the front of the head (figure 7.7(a)) shows that the $I_{z}$ component is coming from the surface towards the inner part of the head.
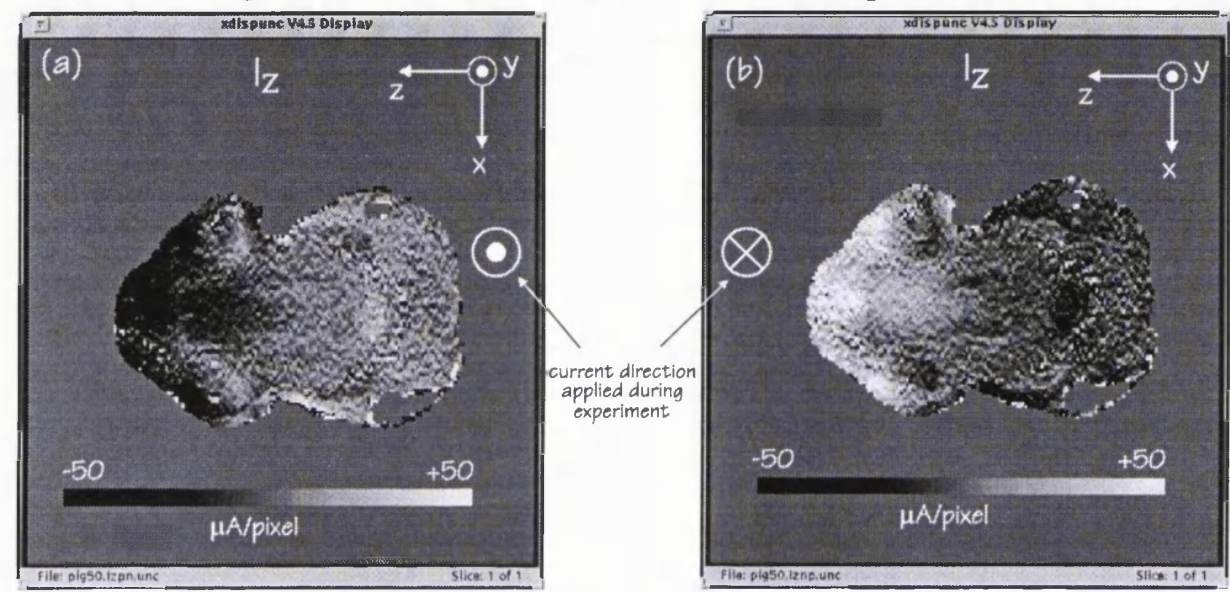

Figure $7.7-I_{z}$ current component images. (a)for the current in the positive y-direction and (b)the negative $y$-direction.

Using the $I_{x}$ (figure 7.5) and $I_{z}$ (figure 7.7) components, a vector plot is show in figure 7.8(a) for the positive and (b) for the negative current. These help to visualize the directions of the current propagation along the slice plane. To plot the vectors the number of pixels in the ROI was reduced with a 3 by 3 mask to average the signal intensity. In the case of the positive current, figure 7.8(a) shows that most of the current in the front of the head is orientated towards the position of the eyes, and is coming out of the eyes in the negative case, figure $7.8(\mathrm{~b})$.
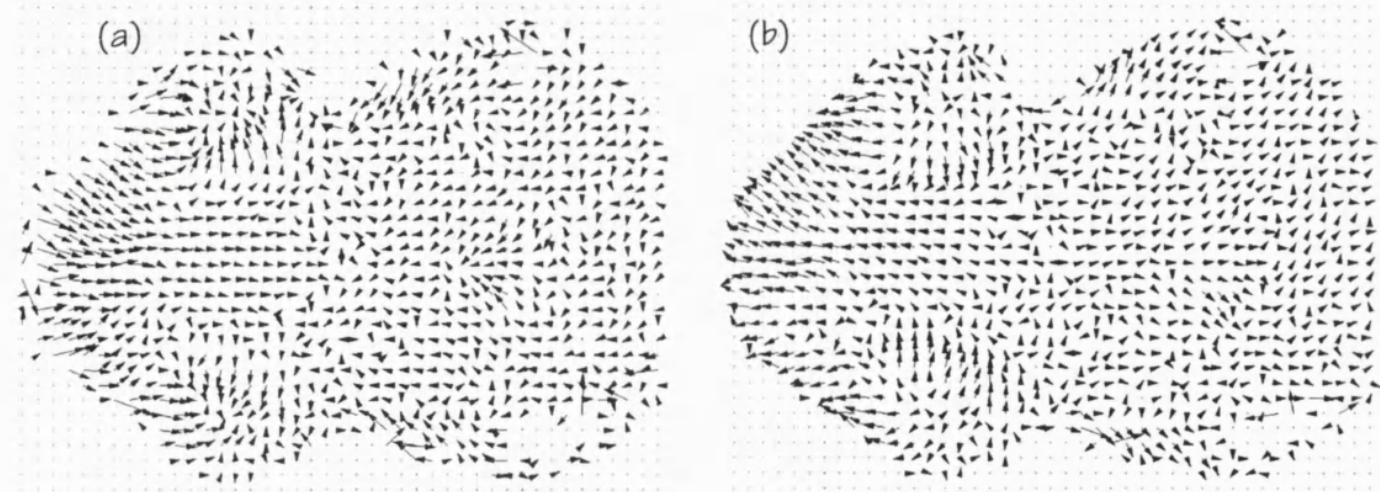

Figure 7.8-Current vector plot. (a) for the PN current and (b) for the NP current.

Using these three components $I_{x}, I_{y}$ (centre) and $I_{z}$, the vector sum or current 
magnitude image was calculated and is shown in figure 7.9(a) for the positive current experiment and figure 7.9(b) for the negative current experiment. Although they appear to be the same, they were computed from two independent NMR experiments.
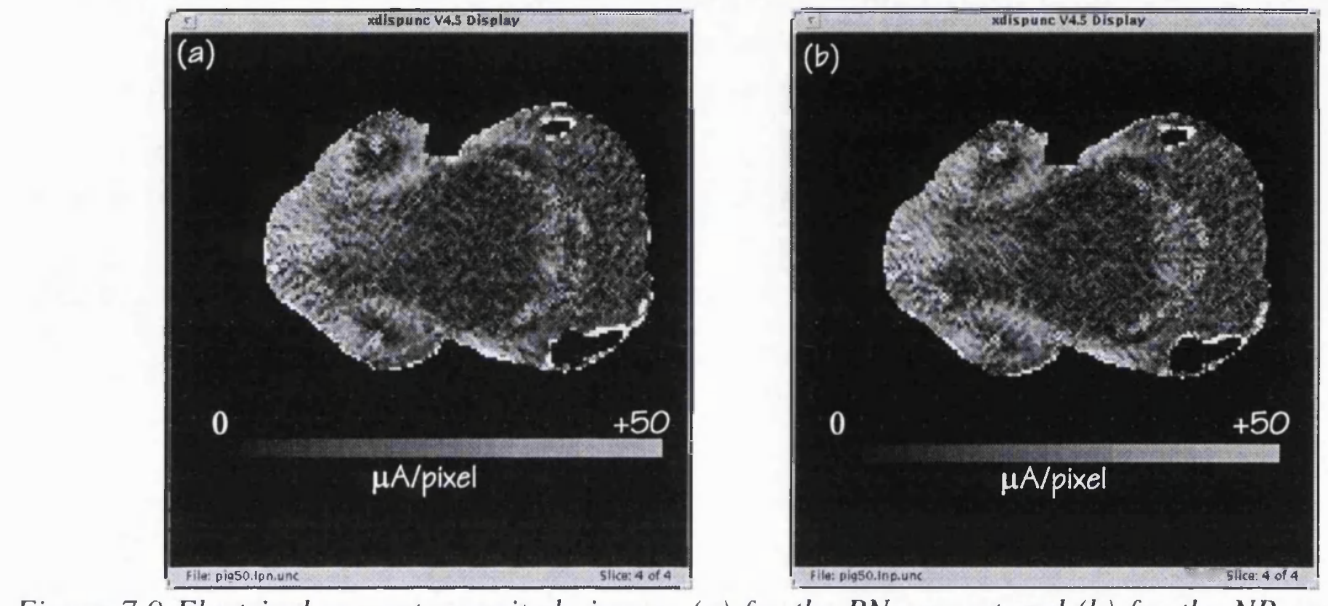

Figure 7.9 Electrical current magnitude image. (a) for the PN current and (b) for the NP current.

\subsubsection{Current per region in the head}

Considering that the electrical current was applied in the $y$-direction, or perpendicular to the tomographic plane, it is very unlikely that the current originating in one electrode would pass through these slices, return back on itself and pass through the slice again. Therefore, making this assumption it should be possible to calculate the total current applied from the surface integral of the image. Also it should be possible to determine the current per sector in the head. To do this one zero-current magnitude image for each slice position was divided into four ROIs as illustrated in figure 7.10. The use of different magnitude images to define the ROIs avoids the problem of anatomical changes due to the $3 \mathrm{~mm}$ distance between the centre of each slice. In figure 7.10 ROI 1 consist of the front of the head and the eyes, ROI 2 is the brain, ROI 3 was defined as the black region between the brain and other regions around it (ie the skull and immediately adjacent tissues) and ROI 4 is the muscles of the neck. The ROIs were defined manually following the pixel contrast. These ROIs were than applied to the $I_{y}$ current images (figure 7.6) to define the surface integration regions. The results for each ROI are given in tables 7.1 for the top slice, 7.2 for the centre slice and 7.3 for the bottom slice. The percentage of the total current applied passing through each region is also given in the tables.

From this first experiment the amount of current that passes through the brain 
at this tomographic position, when the electrodes are place on the top of the head and under the neck, is about $20 \%$ of the total current applied. The differences between the total current applied and the total current measured are about $5.77 \mathrm{~mA}$ or $11.82 \%$ for the top slice, $1.13 \mathrm{~mA}$ or $2.32 \%$ for the centre slice and $0.87 \mathrm{~mA}$ or $1.78 \%$ for the bottom slice. These values are the absolute mean values of the positive and negative current results.
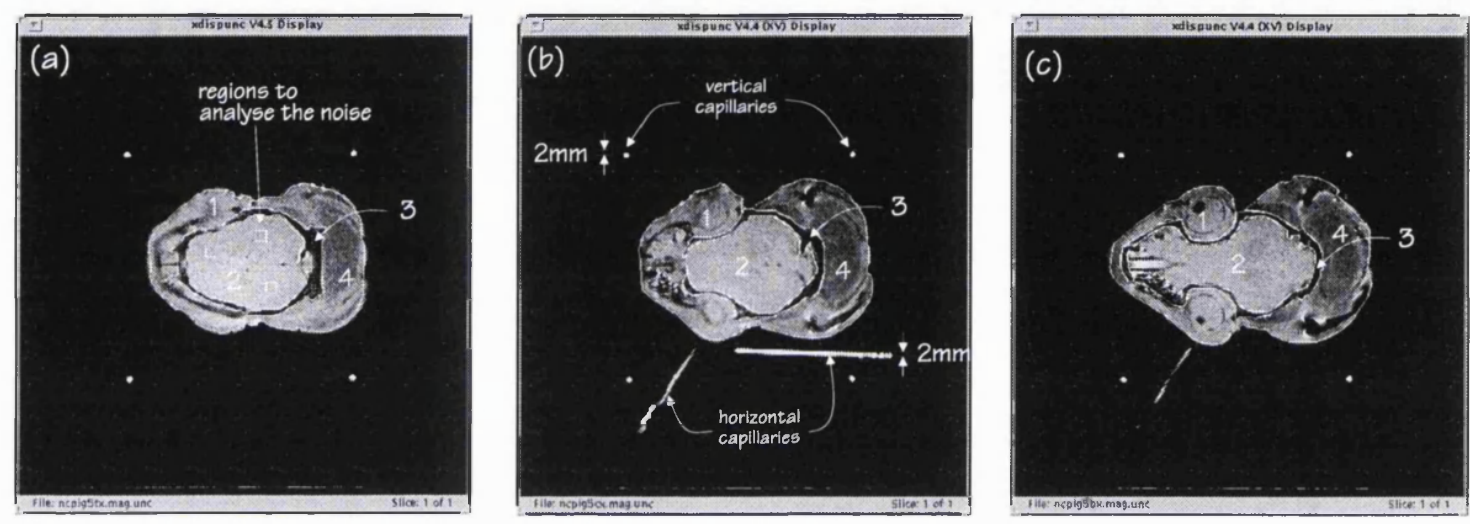

Figure 7.10- ROIs defined to determine the total $1_{y}$ current in the images shown in figure 7.6.

\subsubsection{Results of the second experiment}

The electrical current images from the second piglet head experiment are shown in figure 7.11(b) for the positive current and figure 7.11(d) for the negative current. Just the $y$-component of current was computed in this second experiment. Figure 7.11(a) shows the magnitude image for the top slice acquired with the $x$ axis parallel to $\boldsymbol{B}_{0}$ and figure 7.11(c) shows the magnitude image for the centre slice acquired with the $z$-axis parallel to $\boldsymbol{B}_{0}$.

The magnitude images also show the measured capillary distances used to match the slice positions before and after the rotation. Even though just one rotation of $90^{\circ}$ was done in this experiment, and coronal slices were used for both positions, meaning that we did not need to change the isocentre distance to match the slice position before and after the rotation, there was a difference of $0.87 \mathrm{~mm}$ between the distances indicated in these figures. This may be due to shim artifacts that move the capillary images since they are far from the head centre, or may be due to the precision limitation in the distance measurement since the pixel size is $0.58 \mathrm{~mm}$. 
Table 7.1 - Surface integration results for the top slice.

\begin{tabular}{|c|c|c|c|c|c|c|}
\hline \multicolumn{7}{|c|}{ Top Slice - Current applied $48.8 \mathrm{~mA}$} \\
\hline \multirow{2}{*}{ ROI } & \multirow{2}{*}{$\begin{array}{c}\text { area } \\
\left(\mathrm{mm}^{2}\right)\end{array}$} & \multicolumn{2}{|c|}{$I_{y}(m A)$} & \multirow{2}{*}{$\begin{array}{l}\text { \% of the } \\
\text { current } \\
\text { applied }\end{array}$} & \multicolumn{2}{|c|}{$I_{y}$ area $\left(\mu A / \mathrm{mm}^{2}\right)$} \\
\hline & & positive & negative & & positive & negative \\
\hline$I$ & 976 & 18.69 & -18.41 & $38 \%$ & 19.15 & -18.86 \\
\hline 2 & 1499 & 10.89 & -10.59 & $22 \%$ & 7.26 & -7.06 \\
\hline 3 & 627 & 10.86 & -11.13 & $23 \%$ & 17.32 & -17.75 \\
\hline 4 & 1384 & 14.35 & -14.21 & $29 \%$ & 10.37 & -10.27 \\
\hline & Total & 54.79 & -54.34 & & & \\
\hline & difference & $12 \%$ & $11 \%$ & & & \\
\hline
\end{tabular}

Figure 7.2- Surface integration results for the centre slice.

\begin{tabular}{|c|c|c|c|c|c|c|}
\hline \multicolumn{7}{|c|}{ Centre Slice - Current applied $48.8 \mathrm{~mA}$} \\
\hline \multirow{2}{*}{$R O I$} & \multirow{2}{*}{$\begin{array}{c}\text { area } \\
\left(\mathrm{mm}^{2}\right)\end{array}$} & \multicolumn{2}{|c|}{$I_{y}(m A)$} & \multirow{2}{*}{$\begin{array}{l}\text { \% of the } \\
\text { current } \\
\text { applied }\end{array}$} & \multicolumn{2}{|c|}{$I_{y} /$ area $\left(\mu A / \mathrm{mm}^{2}\right)$} \\
\hline & & positive & negative & & positive & negative \\
\hline 1 & 1425 & 19.65 & -19.29 & 38.99 & 13.79 & -13.53 \\
\hline 2 & 1432 & 9.36 & -8.85 & 18.24 & 6.54 & -6.18 \\
\hline 3 & 481 & 7.29 & -6.67 & 13.98 & 15.15 & -13.87 \\
\hline \multirow[t]{3}{*}{4} & 1648 & 14.38 & -14.37 & 28.79 & 8.73 & -8.72 \\
\hline & Total & 50.68 & -49.18 & & & \\
\hline & difference & $3.71 \%$ & $0.77 \%$ & & & \\
\hline
\end{tabular}

Figure 7.3 - Surface integration results for the bottom slice.

\begin{tabular}{|c|c|c|c|c|c|c|}
\hline \multicolumn{7}{|c|}{ Bottom Slice - Current applied $48.8 \mathrm{~mA}$} \\
\hline \multirow{2}{*}{$R O I$} & \multirow{2}{*}{$\begin{array}{c}\text { Area } \\
\left(\mathrm{mm}^{2}\right)\end{array}$} & \multicolumn{2}{|c|}{$I_{y}(m A)$} & \multirow{2}{*}{$\begin{array}{l}\text { \% of the } \\
\text { current } \\
\text { applied }\end{array}$} & \multicolumn{2}{|c|}{ Ilarea $\left(\mu A / \mathrm{mm}^{2}\right)$} \\
\hline & & positive & negative & & positive & negative \\
\hline 1 & 1203 & 14.06 & -13.72 & $28 \%$ & 11.68 & -11.40 \\
\hline 2 & 1804 & 11.25 & -11.63 & $23 \%$ & 6.24 & -6.45 \\
\hline 3 & 629 & 7.58 & -7.29 & $15 \%$ & 12.05 & -11.59 \\
\hline \multirow[t]{3}{*}{4} & 1825 & 15.28 & -15.06 & $31 \%$ & 8.37 & -8.25 \\
\hline & Total & 48.17 & -47.70 & & & \\
\hline & Difference & $1 \%$ & $2 \%$ & & & \\
\hline
\end{tabular}



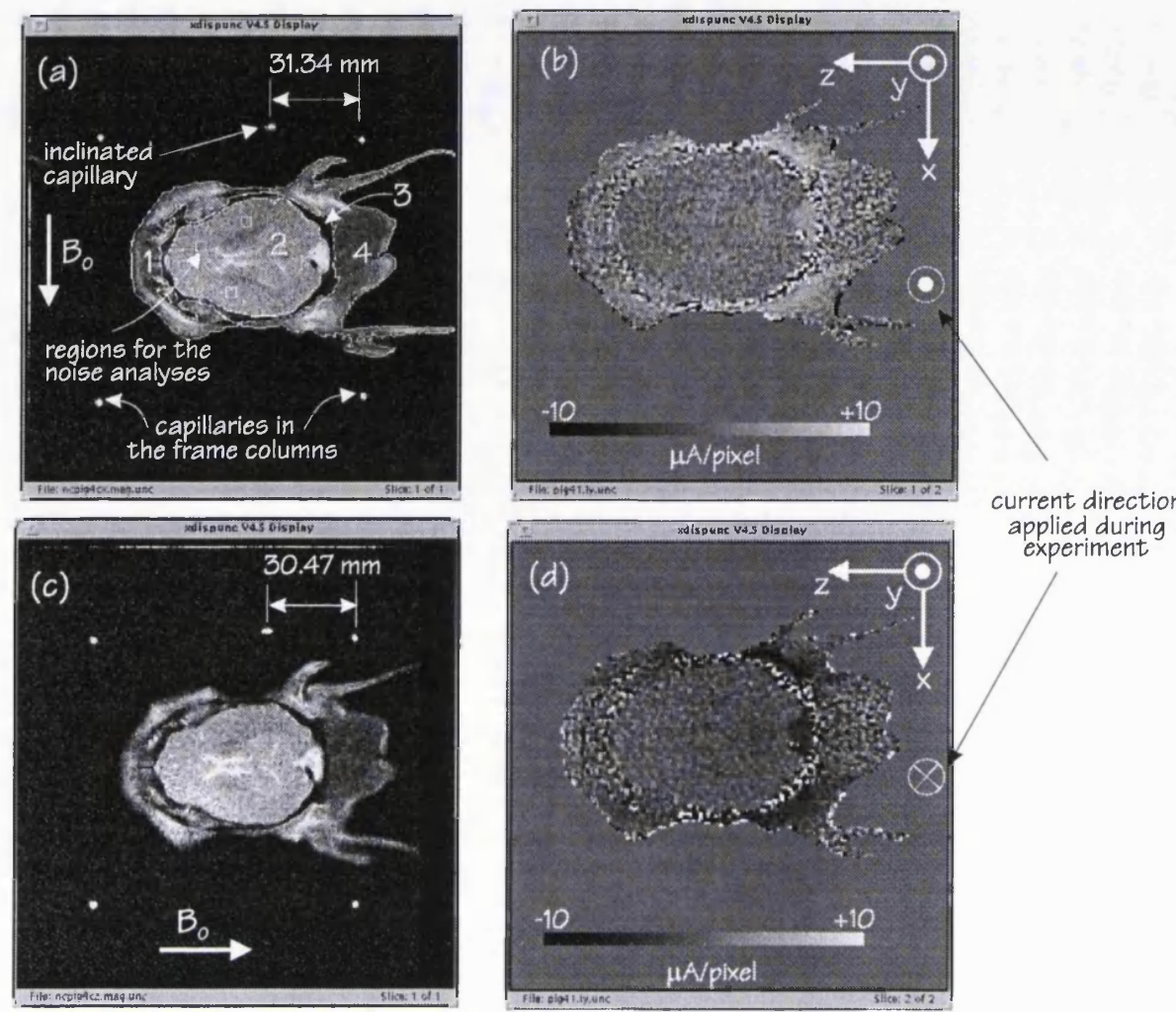

current direction

applied during

Figure 7.11- (a) and (c) NMR images used to adjust the slice position during the experiment. (b) and (d) current images for respectively the positive and negative current applied during experiment.

In figure 7.11 the current images appear noisier than in the previous experiment, (figure 7.6). The reason for this is the signal reduction due to the applied current being halved to $24.4 \mathrm{~mA}$, rather then the standard deviation of the current being increased. Actually these images have a lower noise than the previous experiment as will be shown in section 7.3.

To determine the electrical current per sector in the head the magnitude image in figure 7.11(a) and (c) were divided into four ROIs. As before, ROI 1 consists of the front head, ROI 2 is the brain, ROI 3 is the black region between the brain and other regions around it (ie the skull and immediately adjacent tissues) and ROI 4 is the muscles in the back of the head. The ROIs were again defined manually following the pixel contrast. These regions were then applied to the current images (figures 7.11(b) and (d)) to define the surface integration regions. As before, table 7.4 shows the current integrated per sector of the head in this experiment.

Table 7.4 also gives the sum of the total current integrated per region. In both results, positive and negative current, the sum is equal to $19 \mathrm{~mA}$, which is $20 \%$ less than the total current applied in this experiment $(24.4 \mathrm{~mA})$. This difference may be due to the 
Table 7.4- Surface integral per region for the second experiment. The regions are defined in figure 7.11(a).

\begin{tabular}{|c|c|c|c|c|c|c|}
\hline \multicolumn{7}{|c|}{ Current per sector of the head-Current applied $24.4 \mathrm{~mA}$} \\
\hline \multirow{2}{*}{$R O I$} & \multirow{2}{*}{$\begin{array}{c}\text { area } \\
\left(m m^{2}\right)\end{array}$} & \multicolumn{2}{|c|}{$I_{y}(m A)$} & \multirow{2}{*}{$\begin{array}{l}\text { \% of the } \\
\text { current } \\
\text { applied }\end{array}$} & \multicolumn{2}{|c|}{$I_{y} /$ region $\left(\mu A / m^{2}\right)$} \\
\hline & & $(P N)$ & $(N P)$ & & $(P N)$ & $(N P)$ \\
\hline 1 & 1427 & 6.02 & -6.41 & $25.47 \%$ & 4.21 & -4.49 \\
\hline 2 & 1763 & 6.48 & -6.36 & $26.31 \%$ & 3.68 & -3.61 \\
\hline 3 & 525 & 3.51 & -3.33 & $14.02 \%$ & 6.69 & -6.34 \\
\hline \multirow[t]{3}{*}{4} & 879 & 3.43 & -3.24 & 13.67 & 3.90 & -3.69 \\
\hline & Total & 19.44 & -19.34 & & & \\
\hline & difference & $20 \%$ & $20 \%$ & & & \\
\hline
\end{tabular}

contour area that produces the artifacts seen at the edges of the current image in figure 7.11. The border artifacts are produced by the convolution of the template with the magnetic field images during the process of current calculation. This also happened in the previous experiment, but now for this slice position the current is more concentrated in the regions around the head where this problem exists. As before the border artifacts were removed.

\subsection{In vitro experiments with an intact piglet}

This sub-section describes the only in vitro experiment performed with an intact piglet, rotating the head by 45 degrees.

The piglet used was also provided by the NMR spectroscopy group, but this time the piglet had died during preparation and prior to any hypoxic-ischaemic insult. The study was performed between one and five hours after death.

\subsubsection{Methodology}

\subsubsection{The mechanism to rotate the frame}

To rotate the piglet head and keep it at a known angle during the NMR experiment two squares of perspex $2 \mathrm{~mm}$ thick were fixed on the bottom and left side of the frame illustrated in figure 7.1. Both squares were constructed as illustrated in figure 7.12(a). They each have a hole in the centre plus 9 holes spaced by $11.25^{\circ}$ along a circle of $37 \mathrm{~mm}$ radius. To position the frame, a perspex base, figure 7.12(b), with two pins is fixed onto the NMR trolley. By matching the centre hole and one other with the pins in the base it is possible to lock the frame at a fixed angle with respect to the main 
magnetic field.

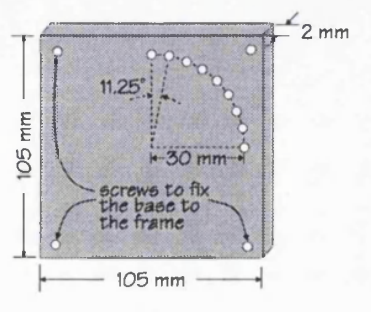

(a)

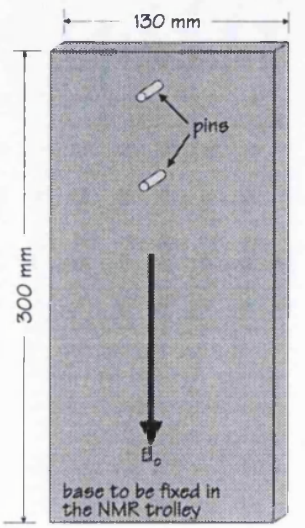

(b)

Figure 7.12 - The mechanism to adjust the head position inside of the magnet

\subsubsection{The electrodes and the electrical current pulses}

The same carbon ECG electrodes used for the isolated head were use in this experiment. The electrodes were placed as before on the top of the head and under the neck, but now this corresponds to the $x$-direction of the current components. The coordinate axes for the whole piglet's body according to the frame position is given in figure 7.1. To reduce the electrical contact resistance, the regions under the electrodes were shaved.

The electrical current amplitude was adjusted to $48.8 \mathrm{~mA}$ with equal periods of $30 \mathrm{~ms}$ for the positive and negative pulse. Therefore the total current time is equal to $60 \mathrm{~ms}$. During the inversion of the spins the current is turned off for $4 \mathrm{~ms}$. This cycle was repeated every $800 \mathrm{~ms}$. Again two different experiment were performed one with current in the $x$-direction, (positive current), and another in the $-x$-direction, (negative current).

\subsubsection{The NMR image parameters}

The NMR image acquisition was a spin-echo pulse sequence with a repetition time of $800 \mathrm{~ms}$, echo-time $60 \mathrm{~ms}$, slice thickness $3 \mathrm{~mm}$, field of view $17 \mathrm{~cm}$, image matrix 256 by 256 with 0.66 by $0.66 \mathrm{~mm}$ pixels.

The tomographic NMR planes were acquired as follow, first with the piglet placed on its side and the neck angled forward, a transverse tomographic plane was acquired ( $x$-axis parallel to $\boldsymbol{B}_{\theta}$ ). Next still with the piglet on its side, but now with the head aligned with its body, a sagittal tomographic plane was acquired (z-axis parallel 
to $\boldsymbol{B}_{0}$ ). Finally the piglet was placed upright and the frame rotated by $45^{\circ}$, a coronal slice was then acquired ( $y$-axis making $45^{\circ}$ with $\boldsymbol{B}_{0}$ ).

Unfortunately for the last slice the piglet's head moved slightly in relation to the frame and just the $I_{x}$ component of the top and centre slices could be computed from the acquired NMR data. This movement was probably caused by the head rotation against a body with rigor mortis.

\subsubsection{The field projection algorithm to determine $B_{y}$}

To compute the $I_{x}$ component of current it is necessary to know the magnetic field in the $y$ - and $z$-direction. Ideally, it is necessary to measure the field components $B_{y}$ and $B_{z}$ by rotating the head strictly by $90^{\circ}$. But since the NMR bore is too small for the rotation of the whole piglet's body, the head was instead inclined in relation to $\boldsymbol{B}_{\boldsymbol{0}}$, and the induced current magnetic field projected along $\boldsymbol{B}_{\boldsymbol{0}}$ was measured.

In figure $7.13, \boldsymbol{B}_{c}$ represents the actual direction of the current induced magnetic field at a certain voxel, $\alpha$ is the angle between the $y$-axis and $\boldsymbol{B}_{0}, \boldsymbol{B}_{z}$ is the field measured with the $z$-axis parallel to $\boldsymbol{B}_{0}, \boldsymbol{B}_{m}$ is the $\boldsymbol{B}_{\boldsymbol{c}}$ projection along $\boldsymbol{B}_{0}$ which is the actual value measured by the NMR experiment. $\boldsymbol{B}_{\boldsymbol{y}}$ is the desired field component in the $y$-direction, which is equal to,

$$
\boldsymbol{B}_{\boldsymbol{y}}=\frac{\boldsymbol{B}_{\boldsymbol{m}}-\boldsymbol{B}_{z} \cos (90-\alpha)}{\cos \alpha}
$$

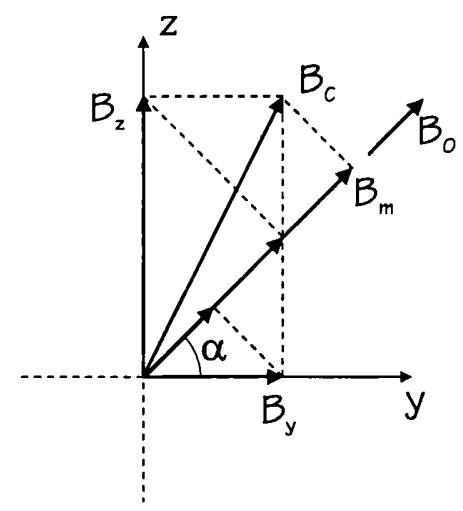

$B_{m}$ is the field measured

$B_{c}$ is the current induce field

$B_{z}$ is the current field component

$B_{y}$ is the current field component

Figure 7.13 - The $B_{y}$ field component calculation.

To compute the $I_{x}$ component, a sub-routine was add to the current program to determine the magnetic field projection along the $y$-axis.

\subsubsection{Results}

Figure 7.14(a) shows the NMR magnitude images and the axis orientation of the 
in vitro experiment body before the images were registered. The gap between the head and the body images is the neck which is off plane. Figure 7.14(b) shows the NMR images after registration. The inclined images are coronal slices. These image were acquired with the piglet upright and the head inclined by $45^{\circ}$ with respect to $\boldsymbol{B}_{0}$. For the images with $z$-parallel to $\boldsymbol{B}_{0}$ the piglet was on its side with the head and body aligned with $\boldsymbol{B}_{0}$, a sagittal slice orientation was used in this case.

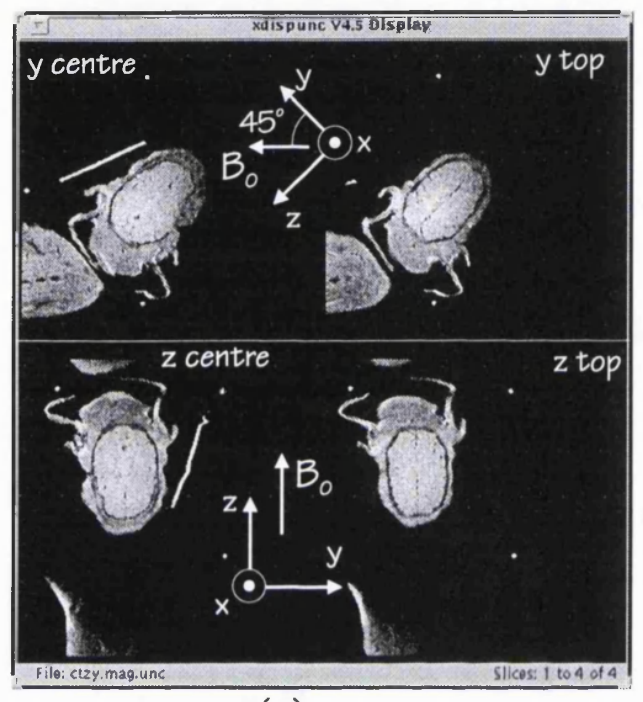

(a)

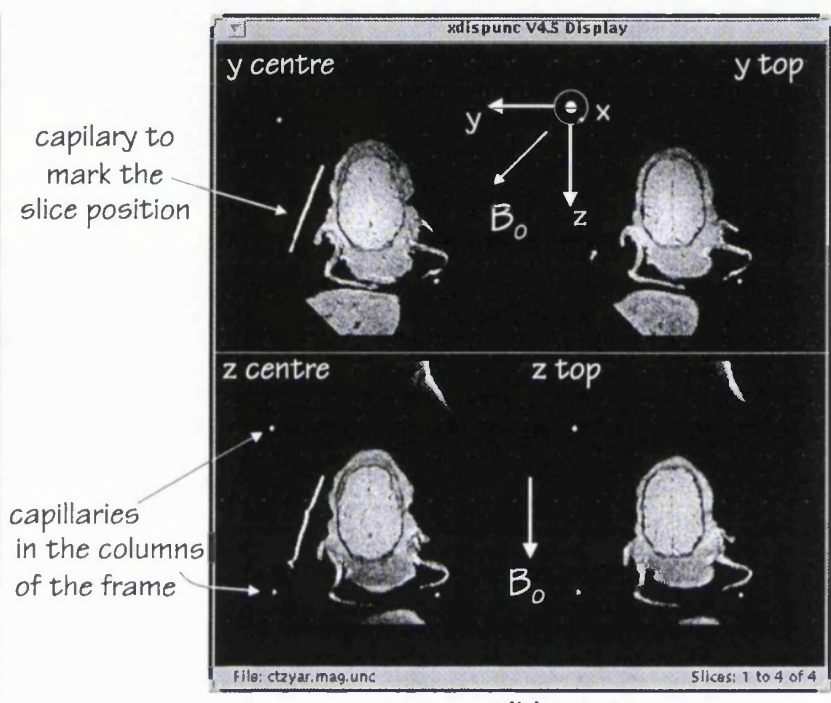

(b)

Figure 7.14 -NMR images to show the orientation of the piglet in relation to the NMR magnetic field $\boldsymbol{B}_{0}$. (a) before (b) after image registration.

Since the piglet is bigger than the FOV, its body image may fold over onto the other side of the image, as shown on the bottom of the images in figure 7.14(a) and (b). To prevent the body image overlapping the head image, the head was moved from the coil centre to the edge. This procedure brings the head to a region where the $\boldsymbol{B}_{\boldsymbol{I}}$ field is not very uniform and the NMR signal intensity in some parts of the image may be affected. If $\boldsymbol{B}_{\boldsymbol{l}}$ is not correct, some of the spins may not be rotated inside of the tomographic plane resulting in a smaller net magnetisation and consequently the NMR signal is reduced. The front end of the images in figure 7.14 of the inclined head shown this signal decrease. Even though the signal may drop, the phase difference between the images with current and without current is not affect by this inhomogeneity, but the image will be noisier since the current standard deviation is directly proportional to the noise to signal ratio.

The centre images in figures 7.14(a) and (b) show the image of the $2 \mathrm{~mm}$ 
diameter capillary used to match the coronal slice acquired with the piglet upright with the sagittal slice acquired with the head on its side.

\subsubsection{Current magnetic field images}

Figures 7.15(a) and (b) respectively show the components $B_{y}$ and $B_{z}$ of the induced magnetic field in the centre and top slices for the positive and negative current. The $B_{v}$ image components in the top and centre slices in this figure were computed using equation (7.1). These images show some artifacts at their edges due to differences between the sagittal and coronal slices. These differences are probably caused by small errors in setting the slice position after the head is rotated. Although the capillaries shown in figure 7.14 appear just in the centre slice it is necessary to consider that the capillary is $2 \mathrm{~mm}$ in diameter and the slice thickness is $3 \mathrm{~mm}$ which leaves a possible error of $1 \mathrm{~mm}$. Also the skin around the head may move when the rotation is performed.
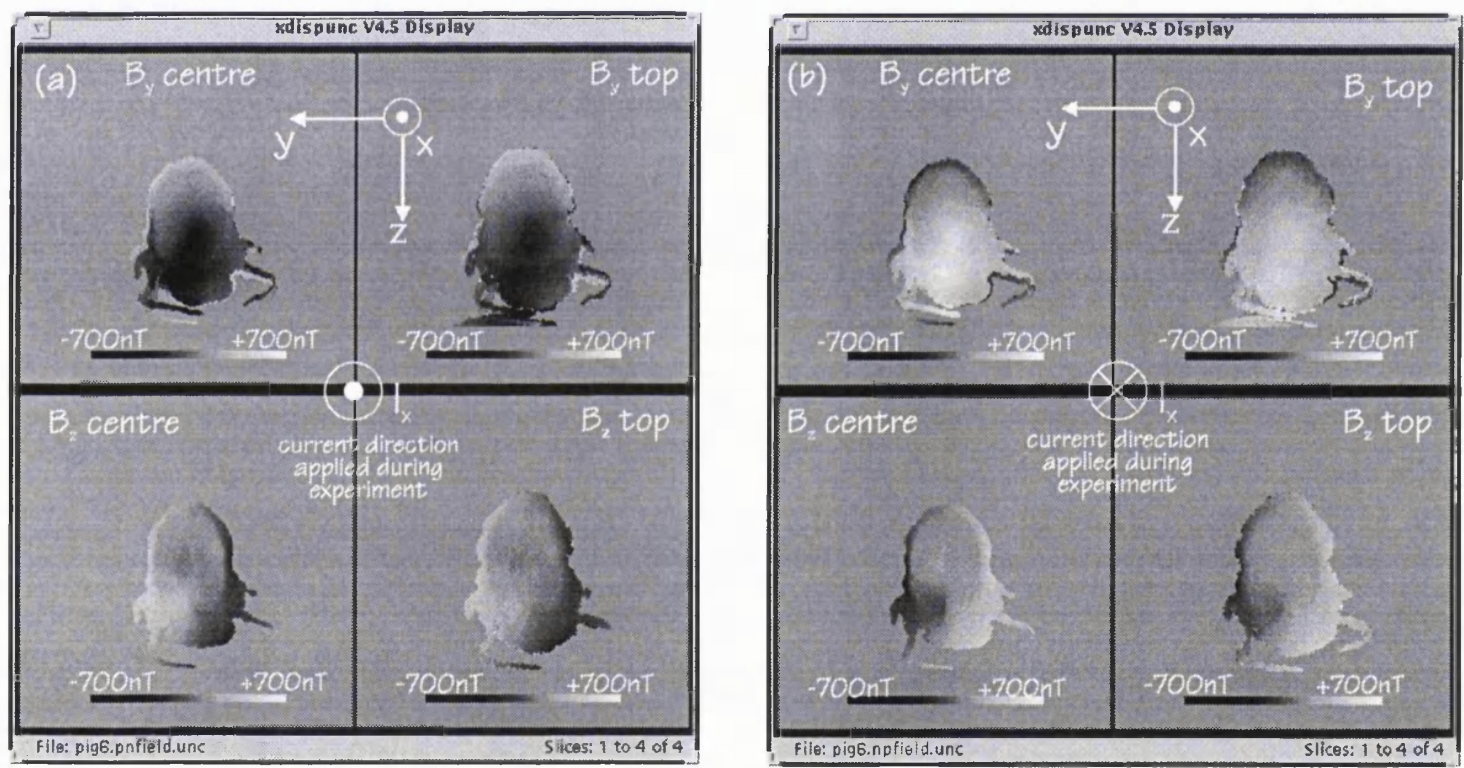

Figure 7.15-Current magnetic field images (a)positive current experiment (b)negative current experiment.

\subsubsection{Electrical current images}

Figure 7.16 shows the image of the electrical current component in the $x$ direction of the top and centre slices. Figures 7.16 (b) and (e) are the top and centre slices for the positive (PN current sequence pulses), and figures 7.16 (c) and (f) are the top and centre slices for the negative current (NP current sequence pulses). Only the $I_{x}$ current component for the top and centre slices are shown because the piglet head moved at the end of this experiment and the other components could not be calculated. 

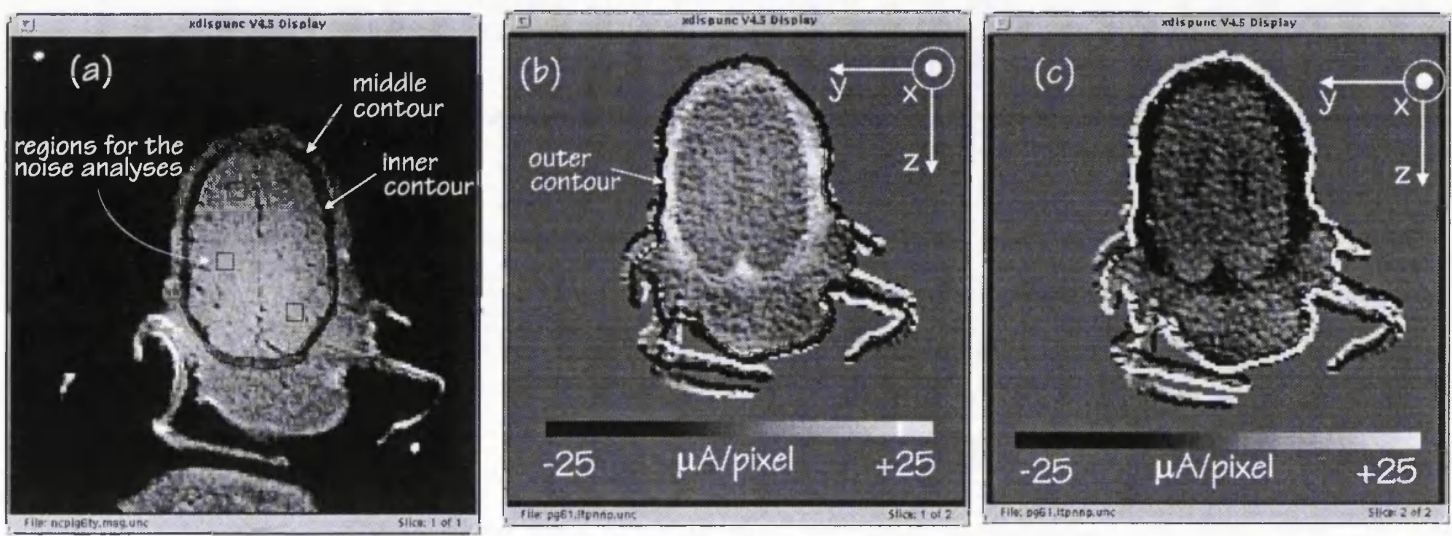

current direction

- $\begin{gathered}\text { applied during } \\ \text { experiment }\end{gathered}$
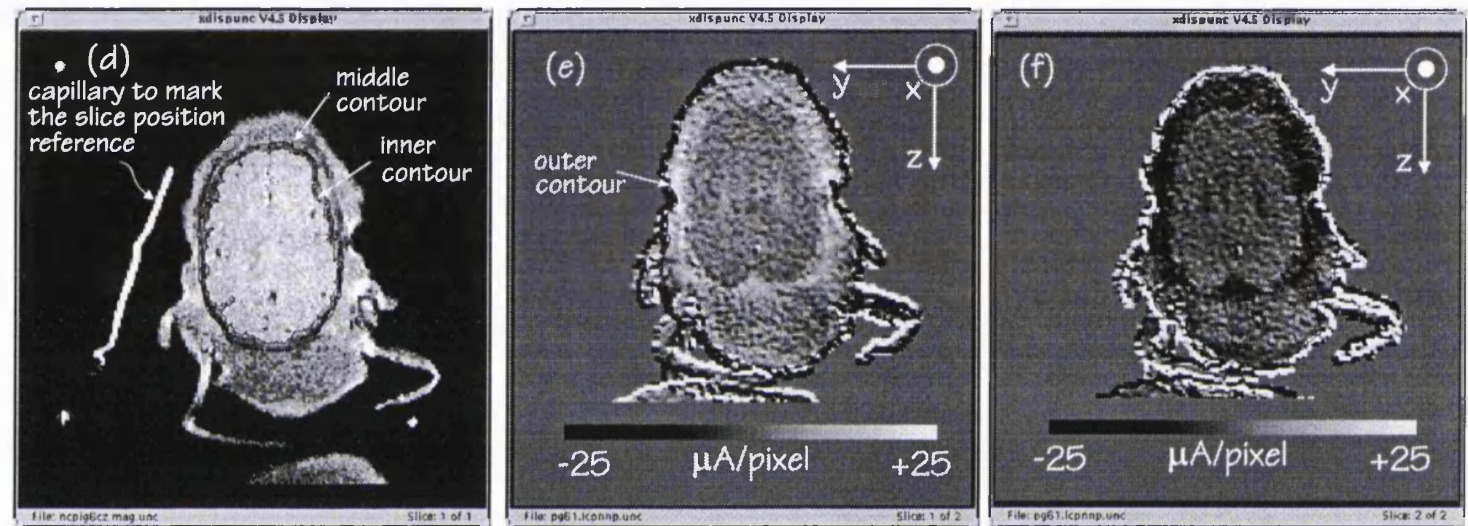

Figure 7.16- (a) and (d) NMR images, (b) and (e) positive current, (c) and (f) negative current, respectively top and centre slices.

\subsubsection{Current per region in the head}

The magnitude images of the top and centre slices shown in figure 7.16(a) and (b) were divided into three regions. The inner contour defines the region of the brain alone, the middle contour defines the region of the brain plus the skull and the tissues nearby it. The outer contour encompassed the whole head and was drawn using figures 7.16(a) and (b) to try and avoid the edge artifacts in the current images. For each region the integrated current is given in tables 7.5 for the top slice and 7.6 for the centre slice.

\subsection{Noise analyses in the in vitro current images}

To determine the level of noise in the current images, a noise analysis was performed as described in section 6.3. For each experiment a magnitude image was selected in which to place three sample regions of 6 by 6 pixels. Figure 7.10(a) and 7.11(a) show the ROI positions for the in vitro experiments with isolated head, figure 7.16(a) show the ROI positions for the whole body experiment. All three regions were placed in the brain areas where the current is most uniform. In this way it is possible 
Table 7.5 - Surface integration results for the top slice. The ROIs are defined in figure 7.16(a).

\begin{tabular}{|c|c|c|c|c|c|c|}
\hline \multicolumn{7}{|c|}{ Top Slice - Current applied $48.8 \mathrm{~mA}$} \\
\hline \multirow{2}{*}{ ROI } & \multirow{2}{*}{$\begin{array}{c}\text { area } \\
\left(\mathrm{mm}^{2}\right)\end{array}$} & \multicolumn{3}{|c|}{$I_{y}(m A)$} & \multicolumn{2}{|c|}{$I_{y} /$ area $\left(\mu \mathrm{A} / \mathrm{mm}^{2}\right)$} \\
\hline & & positive & negative & $\begin{array}{l}\text { \% of the } \\
\text { current } \\
\text { applied }\end{array}$ & positive & negative \\
\hline $\begin{array}{c}\text { inner contour } \\
\text { (brain) }\end{array}$ & 1413 & 11.38 & -11.90 & 23.85 & 8.05 & -8.42 \\
\hline $\begin{array}{c}\text { middle contour } \\
\text { (brain plus skull) }\end{array}$ & 1654 & 18.49 & -18.59 & 37.99 & 11.18 & -11.24 \\
\hline outer contour & 2825 & 34.00 & -32.93 & 68.58 & 12.04 & 11.66 \\
\hline $\begin{array}{c}\text { skull } \\
\text { (middle - inner) }\end{array}$ & 241 & 7.11 & -6.69 & 14.14 & 29.50 & -27.76 \\
\hline $\begin{array}{c}\text { muscle } \\
\text { (outer - middle) }\end{array}$ & 1171 & 15.51 & -14.34 & 14.93 & 13.25 & -12.25 \\
\hline
\end{tabular}

Table 7.6 - Surface integration results for the centre slice. The ROIs are defined in figure 7.16(d).

\begin{tabular}{|c|c|c|c|c|c|c|}
\hline \multicolumn{7}{|c|}{ Centre Slice - Current applied $48.8 \mathrm{~mA}$} \\
\hline \multirow{2}{*}{ ROI } & \multirow{2}{*}{$\begin{array}{c}\text { area } \\
\left(\mathrm{mm}^{2}\right)\end{array}$} & \multicolumn{3}{|c|}{$I_{y}(m A)$} & \multicolumn{2}{|c|}{$I_{y} /$ area $\left(\mu \mathrm{A} / \mathrm{mm}^{2}\right)$} \\
\hline & & positive & negative & $\begin{array}{l}\text { \% of the } \\
\text { current } \\
\text { applied }\end{array}$ & positive & negative \\
\hline $\begin{array}{c}\text { inner contour } \\
\text { (brain) }\end{array}$ & 1460 & 9.08 & -8.73 & 18.24 & 6.22 & -5.98 \\
\hline $\begin{array}{l}\text { middle contour } \\
\text { (brain plus skull) }\end{array}$ & 1729 & 13.38 & -13.08 & 27.11 & 7.74 & -5.57 \\
\hline outer contour & 3313 & 34.50 & -29.26 & 65.33 & 10.41 & 8.83 \\
\hline $\begin{array}{c}\text { skull } \\
\text { (middle - inner) }\end{array}$ & 269 & 4.30 & -4.35 & 8.86 & 15.98 & -16.17 \\
\hline $\begin{array}{c}\text { muscle } \\
\text { (outer-middle) }\end{array}$ & 1584 & 21.12 & -16.18 & 38.21 & 13.33 & -10.21 \\
\hline
\end{tabular}

to compare the predicted current standard deviation using equation (6.28) with the actual value measured in the current image. Table 7.7 shows the noise analyses results.

For the first experiment the overall average of the current standard deviation is equal to $2.57 \mu \mathrm{A}$ for the second $1.24 \mu \mathrm{A}$ and for the whole body experiment $2.57 \mu \mathrm{A}$. The predicted value and the measure values in table 7.7 show a very good agreement.

An interesting point to note in these results is the difference between the two isolated head experiments. Comparing the images in figures 7.6 and 7.11 with the noise results we should expected a much noisier image in the former which has an average 
current deviation of $2.57 \mu \mathrm{A}$ compared with the latter which has a value of about $1.24 \mu \mathrm{A}$. Even though the NMR signal is lower for long echo-time, the parameters for the second experiment demonstrated that a lower noise current image can be obtained using a current-spin integration time of about $120 \mathrm{~ms}$ instead of $60 \mathrm{~ms}$.

The main reason for the noisier image in figure 7.11 is that the total current applied was halved to $24.4 \mathrm{~mA}$. Table 7.7 gives the mean value of current for each ROI selected for the noise analyses. The mean current difference between the two

Table 7.7 - Results of the noise analysis in the in vitro piglet experiments.

\begin{tabular}{|c|c|c|c|c|c|c|c|}
\hline \multirow{2}{*}{ experiment } & \multirow{2}{*}{$R O I$} & \multirow{2}{*}{\multicolumn{2}{|c|}{${ }^{*} I \mu A$}} & \multicolumn{3}{|c|}{${ }^{* *} \sigma(I) \mu A$} & \multirow{2}{*}{${ }^{* * *}<M>/ \sigma(M)$} \\
\hline & & & & \multicolumn{2}{|c|}{ measured } & predicted & \\
\hline \multirow{3}{*}{$\begin{array}{c}\text { Ist } \\
\text { experiment } \\
\text { ROI in figure } \\
7.10\end{array}$} & 1 & 2.59 & \multirow{3}{*}{${ }^{(\bullet)}<2.51>$} & 2.95 & \multirow{3}{*}{$@(@)<2.57>$} & 3.35 & 18.12 \\
\hline & 2 & 2.95 & & 2.25 & & 2.98 & 20.33 \\
\hline & 3 & 2.00 & & 2.47 & & 2.18 & 27.69 \\
\hline \multirow{3}{*}{$\begin{array}{c}2 n d \\
\text { experiment } \\
\text { ROI in figure } \\
7.11\end{array}$} & 1 & 1.44 & \multirow{3}{*}{${ }^{\circledR}<0.85>$} & 1.16 & \multirow{3}{*}{ 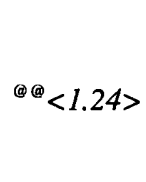 } & 1.17 & 10.89 \\
\hline & 2 & 0.57 & & 0.99 & & 1.28 & 9.93 \\
\hline & 3 & 0.55 & & 1.50 & & 1.13 & 11.23 \\
\hline \multirow{3}{*}{$\begin{array}{c}3 \mathrm{3rd} \\
\text { experiment } \\
\text { ROI in figure }\end{array}$} & 1 & 1.71 & \multirow{3}{*}{ (i) $<3.20>$} & 2.68 & \multirow{3}{*}{ (1) (1) $<2.57>$} & 1.97 & 14.58 \\
\hline & 2 & 2.53 & & 2.66 & & 2.27 & 12.66 \\
\hline & 3 & 5.34 & & 2.36 & & 1.72 & 16.68 \\
\hline
\end{tabular}

\footnotetext{
* ROI - current average

** ROI - current standard deviation

*** ROI - NMR magnitude average divided by the standard deviation

average of the three values

(a) average value $=\left[\left(\sum x^{2}\right) / 3\right]^{1 / 2}$

$1 T E=60 \mathrm{~ms}, T R=1000 \mathrm{~ms}$, pixel size $0.70 \times 0.70 \mathrm{~mm}$, current applied $48.8 \mathrm{~mA}$

$T E=120 \mathrm{~ms}, T R=1200 \mathrm{~ms}$, pixel size $0.59 \times 0.59 \mathrm{~mm}$, current applied $24.4 \mathrm{~mA}$

$T E=60 \mathrm{~ms}, T R=800 \mathrm{~ms}$, pixel size $0.66 \times 0.66 \mathrm{~mm}$, current applied $48.8 \mathrm{~mA}$
}

experiments is about a factor of three while the noise standard deviation is about two, which explain the slightly noisier image.

Comparing the first experiment with isolated head and the third experiment with the whole body, both were performed using the same applied current and current-spin integration time, and the noise level is basically the same. The mean current value difference of the three ROI between these two experiment is about $27 \%$ which is not a large difference considering that the slices are not exactly at the same position, see 
figures 7.2 and 7.16 .

\subsection{Discussion}

A total of six in vitro experiments were performed, of which three were described here. The results of the first three experiments were not presented because we had problems in accurately positioning the frame to hold the piglet head in the centre of the NMR magnet and in rigidly clamping the head into the frame. The design of the frame and the head clamps was modified during the course of these first three experiments.

The reasons for placing the electrodes in the top of the head and under the neck and selecting coronal slices through the head was to enable images to be taken of the regions around the skull were there are more surface tissues. These regions theoretically are where most of the applied electrical current would propagate, since the skull has a very low conductivity. In one of the first experiments, not presented here we tried to place the electrodes between the ears and the eyes and acquire a coronal slice near the midline region of the brain. However, the region on the top of the head between the skin and skull is very thin and because of the edge artifacts produced by the template convolution most of the data in this area is lost during the image calculation. For this reason it was not possible to produce the current density images in the tissues near the surface of the head.

In EIT the tomographic plane is in the plane of the electrodes. It is possible to acquire NMR slices in the same plane as the electrodes, but there will be considerable problems with the phase unwrapping procedure near the electrodes. Near to the electrodes the current density is very high and probably the phase will wrap by more than $180^{\circ}$ per pixel and hence the actual unwrapped phase cannot be recovered. Although it would still be possible to measure the current distribution in the brain, where lower current density is expected.

A major problem during the animal experiments is to accurately identify the slice positions acquired before the rotation, particularly when the slice orientation is changed. The use of capillaries to mark the starting position and to define the tomographic plane was found to be the best solution to overcome this problem, since it does not require any calculation or measurement during the experiment. All we need is after the rotation is to look for the capillaries in order to get back to the starting position. Even so, this 
is not a perfect matching technique as can be seen from the first experiment, which gave the smallest difference between the total integrated current and the current applied. In figure 7.2 each row should show exactly the same set of images, but for the $y$ orientation images (b), (e) and (h) they show small anatomic differences when compared to the $x$ - and $z$-orientations, which are much more similar to each other. The reason for the mis-positioning may be due to errors caused by the capillaries being $2 \mathrm{~mm}$ in diameter compared with the $3 \mathrm{~mm}$ slice thickness. Also, we have to consider that this region of the head is much more subject to anatomic changes than the regions above.

For the first experiment, this slice mis-position error affects just the $I_{x}$ and $I_{z}$ components which need data from both head orientations and from all three slices for their calculation. On the other hand, calculation of the $I_{y}$ component just needs one slice position for the start orientation and one after the first rotation. In this case for both orientations the slices were coronal. This fact explains the very small difference between the sum of the current integrated per region and the current applied during the experiment in tables 7.1, 7.2 and 7.3.

In the second experiment with the isolated head, the current applied was $24.4 \mathrm{~mA}$, but with a longer current-spin integration time of $120 \mathrm{~ms}$. The resulting current image was not as good as in the first experiment. As demonstrated by the noise analyses, this was not caused by an increase in the noise level of the images. The reason for the image degradation was due to the lower current applied. As shown in table 7.7 the level of current is too small for the experiment.

Considering now the results for the whole body experiment. The first point to consider in this experiment is that it was performed soon after death and there should have been little damage to the structure of the head. This experiment did prove that it is still possible to measure in tissue the current distribution by rotating the head by just $45^{\circ}$ instead of the ideal $90^{\circ}$. The images are quite reasonable and still show the current distribution in a plane orthogonal to that of the electrodes used to apply the electrical current. Unfortunately the piglet's head moved at the end of the experiment and the other components could not be computed.

The total current integrated using the outer contour, (tables 7.5 and 7.6), shows that just $67 \%$ of the total current applied was actually measured. This difference is probably due to the fact that the current in this slice position was much more 
concentrated in the tissues around the surface of the head. Since this is a region in which it is not possible to measure the electrical current it may well explain a large part of this difference. The same dead zone exists in the first experiment, but in this case most of current is concentrated around the eyes as seen in figure 7.6. The other source of error arises from the small rotation which only gives rise to a magnetic field which is about $70 \%$ of the $B_{y}$ component. Thus, some values of $B_{y}$ may have been too small to be measured by the NMR experiment. Another possible source of error in the total current integrated compared with the current applied during the experiment is the use of two different slices orientations to measure the magnetic field. But even so this error is small if the difficulties of acquiring exactly the same tomographic plane before and after rotation.

Considering just the current that propagates in the brain, in the first experiment with the isolated head, tables $7.1,7.2$ and 7.3 show that about 18 to $23 \%$ of the total applied current goes through the brain. In the second experiment (table 7.4) the corresponding value was about $26 \%$. The experiment for the whole body (tables 7.5 and 7.6) give for the top slice $24 \%$ and for the centre slice $18 \%$. These values are consistent with the results obtained in chapter 8 for the post mortem experiment table 8.2.

Considering now the accuracy of these measurements. This is highly dependent on the technique used to match the slices position before and after rotation rather than on the noise level in the current image. Comparing the total integrated current in the head with the actual current applied, for the first experiment the difference for each slice is $11.5 \%, 2.24 \%$ and $1.5 \%$, for the second experiment $20 \%$ and for the third experiment $31 \%$ (all values are the mean value of the positive and negative results). From these differences between the total current integrated and the total current applied it is possible it is very unlikely that an absolute accuracy of better than 20 to $30 \%$ can be achieved.

When discussing the current images, just the $I_{y}$ component is integrated to give a result that can be compared with the actual current applied during the experiment. The integration of the $I_{x}$ and $I_{z}$ components can give any value, since the current may flow along many pixels within the slice before going out of the plane in the direction of the top or bottom electrode. This leads to the question: is it really necessary to measure the $I_{x}$ and $I_{z}$ components ? The answer is yes, and the reason can be seen if the magnitude of the current image, (figure 7.9), is compared with just the $I_{y}$ component, (figure 7.6). 
Looking just at figure 7.6 we can see that regions where $I_{y}$ has a very low or almost zero value there is a very high component in the $z$-direction. Therefore the right approach to visualise the total current distribution in the head is to use the magnitude image which shows exactly how the total is distributed along the tomographic plane.

\subsection{Conclusion}

From the in vitro experiments we conclude that the minimum applied current required for this experiment is around $50 \mathrm{~mA}$. Ideally this value showed be slightly bigger, perhaps about $60 \mathrm{~mA}$. The ideal current-spin time integration for the piglet experiment is around $100 \mathrm{~ms}$ which would make the inherent MR image noise less significant. This value is however very dependent on the $T_{2}$ value which may be different for a live piglet.

The results of the in vitro experiment with both the isolated head and the whole body showed that about $17 \%$ of the total current applied to the head goes through the brain when the electrodes are placed on the top of the head and under the neck. This value maybe still different for a live piglet although it does not look like major differences will occur. The accuracy of the current measurement in these experiment was around $20 \%$. 


\section{Chapter 8}

\section{Results : In vivo Current Images in the Piglet Head}

\section{In vivo experiment with a newborn piglet}

In this chapter two experiments performed with the same newborn piglet are presented. The first experiment described was performed in vivo on the anaesthetised animal and the second, soon after death. In both experiments the same procedure was applied. The purpose of this study was to determine if the current distribution previously measured on a post-mortem pig head were representative of those that would be found in the intact live animal, and if they were not, then to try and identify the major causes of the observed differences.

This chapter starts by describing the animal preparation for the in vivo experiment, the electrode positions, electrical current applied and the NMR image parameters used to acquire the data. The results are divided into two parts, those obtained with the animal alive and those after death. The chapter ends with a discussion and conclusion.

\subsection{Animal preparation}

The in vivo experiment was performed on a one day old newborn piglet (weight $1.9 \mathrm{~kg}$ ). The animal was anaesthetised with an initial intraperitoneal dose of $10 \mathrm{ml}$ of urethane $(36 \% \mathrm{w} / \mathrm{v})$ which was complemented with further doses of intravenous urethane $(1 \mathrm{ml}, 24 \% \mathrm{w} / \mathrm{v})$, approximately every 15 minutes. An umbilical vein was cannulated to apply the complementary doses. Three hours after induction of anaesthesia the animal was given $5 \mathrm{ml}$ of gelofusin to help maintain blood pressure. During the experiment the ECG and temperature were monitored. The ECG was measured with three carbon electrodes placed in the chest of the piglet and the temperature with a rectal thermistor.

During initial tests (explained further in section 8.2) to determine the amplitude of the current pulses to be applied in the experiment, it was noticed that the neck and throat muscle stimulation caused by the electrical current was interfering with the breathing, and a tracheotomy was therefore performed to ensure a constant open airway.

Once the animal was anaesthetised its head was fixed in the frame described in 
the last chapter. The head was held by the two ceramic points which were firmly screwed against the skull in the regions between the eyes and ears. The nose was held by the front bar which was placed in the mouth and held in position by pressure from a second bar on the top of the snout.

To keep the piglet warm inside the NMR machine its body was wrapped with cotton wool, over which was placed a sheet of aluminum to improve the thermal isolation. At the beginning of the experiment the rectal temperature was $38.4^{\circ}$ celsius and at the end $37.8^{\circ}$ celsius. The piglet preparation took approximately three hours and the experiment was completed within seven hours. At the end of the experiment the animal was killed with an intravenous injection of $\mathrm{KCl}$.

\subsection{The electrodes and the electrical current}

During the in vitro experiments it had been concluded that for optimum $\mathrm{S} / \mathrm{N}$, the electrical current to be applied in this type of experiment should ideally be more than $50 \mathrm{~mA}$, and the current-spin integration time more than $60 \mathrm{~ms}$. The actual current amplitude that could be applied in this in vivo experiment would however depend on the electrode positions, and on how the animal would respond to the current pulses.

The current-spin integration time used in the in vivo experiment was set at $80 \mathrm{~ms}$. The choice of this value was based on two reasons: firstly, the $T_{2}$ relaxation time in the cerebral tissues of newborn piglets was measure found to be $80 \mathrm{~ms}$ in experiments carried out by the NMR group here at Medical Physics; and secondly, the results of the in vitro experiments (section 7.4) suggested that a period of more than $60 \mathrm{~ms}$ should be used to reduce the standard deviation in the calculated current images.

To determine how much current could be applied, three electrodes were placed on the head: one on the top of the head (which was the reference electrode and connected to the current source), one under the neck (as was done in the in vitro experiments) and another electrode on the inside roof of the mouth. The electrodes used were the same ECG carbon electrodes used in the in vitro experiment, and the skin on the top of the head and under the neck of the animal was shaved to improve the electrical contact. To ensure that the electrodes would not come detached during the experiment, cyanoacrylate adhesive was applied around the periphery of the electrodes.

The electrical current pulses were first applied using the electrode under the neck. As the amplitude of the electrical current was increased beyond approximately 
$24.4 \mathrm{~mA}$, it was noticed that the animal's breathing became more difficult. Since no muscle movements were observable it was concluded that the electrical current was probably causing spasms in the trachea thus restricting the entrance of air. The same effect was noticed when the electrode on the roof of the mouth was used. Therefore a tracheostomy was performed to insert an endotracheal tube and hence relieve any obstruction and provide a constant airway for respiration. With the endatracheal tube in place, it was possible to increase the applied current to $60 \mathrm{~mA}$ without causing any difficulties in breathing. However, if the neck electrode was used at this level of applied current, there was visible twitching of the muscles in the neck and throat. These stimulation effects were much smaller when the electrode in the roof of the mouth was used.

Ideally we would prefer to use the same electrode positions as used for the in vitro experiments, so that both sets of results in principle could be compared, but to avoid muscle stimulation in the neck, it was decided to use the electrode inside of the mouth.

Using the electrodes on the top of the head and inside the mouth, it was possible to use a current amplitude of $60 \mathrm{~mA}, 10 \mathrm{~mA}$ higher than in the in vitro experiments. It was hoped that this increase in current would provide an improved SNR in the current images, especially since with the second electrode on the roof of the mouth, more current would be expected to propagate through the brain. The current was divided into two symmetrical pulses of $40 \mathrm{~ms}$, repeated every second.

The approximate electrodes position are indicated in a sagittal image shown in

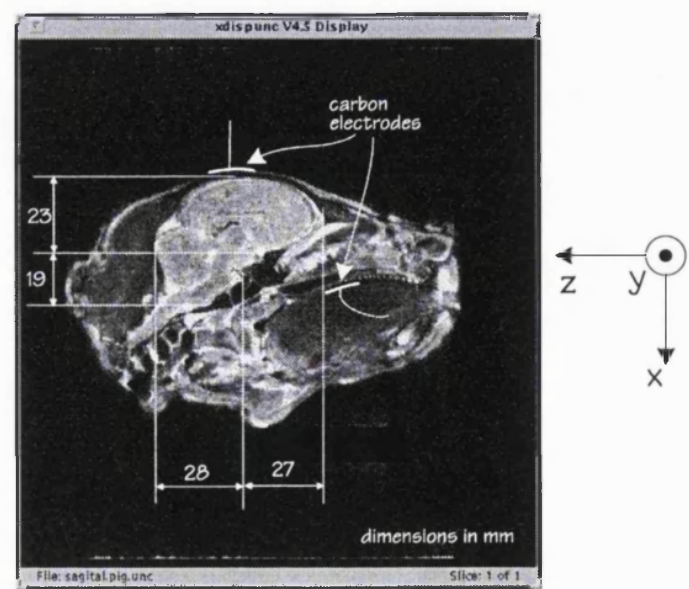

Figure 8.1-Sagittal image of the piglet head to approximately show the positions of the electrodes and the brain dimensions. 
figure 8.1. This image is not from this experiment, but from a previous in vitro experiment performed at the beginning of the experimental work, which is not presented in this thesis.

The electrical current was applied in the $x$-direction of the axis reference as shown in figure 8.1. To reduce the time required to perform the experiment, we only acquired images with a positive-negative current sequence.

\subsection{The frame holder modifications}

During the simulations we found that to avoid errors in the current image the magnetic field produced by the conductors near to the frame should be measured. Copper wires were fixed to the frame and the carbon cables from the electrodes were connected to these. The copper conductors around the frame were fixed using superglue at the positions shown in figure 8.2. The current supply was then connected to the copper conductors in the frame via a pair of twisted wires.
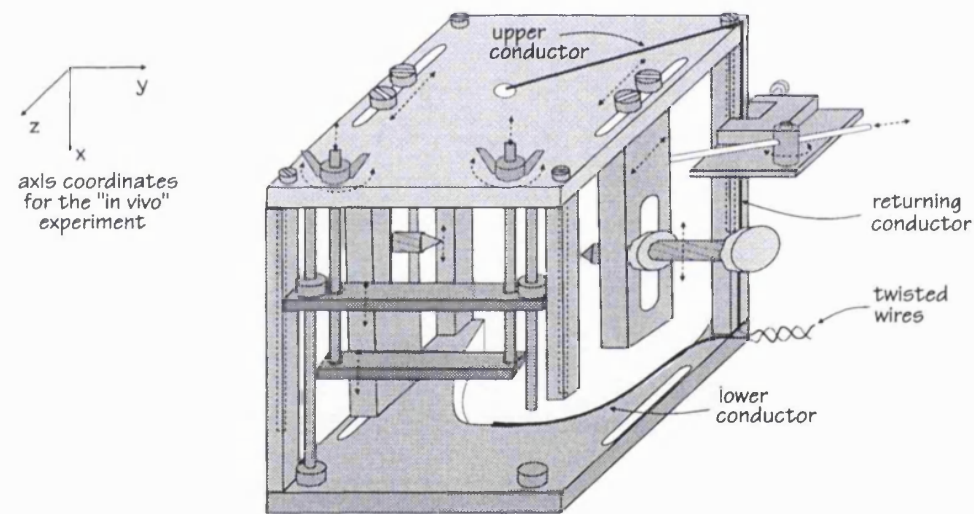

Figure 8.2 - Position of the copper conductors in the frame holding the piglet head (one of the front support bars is shown cut away for clarify).

\subsection{The NMR image parameters}

The spin-echo time used for the in vivo experiment was set to a repetition time of $1000 \mathrm{~ms}$, echo-time of $80 \mathrm{~ms}$ (equal to the total current time), slice thickness $3 \mathrm{~mm}$ and field of view of $16 \mathrm{~cm}$. The isocentre distances were set to $-23 \mathrm{~mm}$ for the top slice, $-20 \mathrm{~mm}$ for the centre slice and $-17 \mathrm{~mm}$ for the bottom slice, giving a sequence of three adjacent slices. 256 by 256 image matrix with pixels of 0.63 by $0.63 \mathrm{~mm}$ were used in both transverse and coronal slices. The scan time for each image in these experiment was 5.20 minutes. To position and hold the frame inside the magnet the same perspex base shown in figure 7.12 was used. The frame was placed first with the $z$-axis parallel 
to $\boldsymbol{B}_{0}$, then it was rotated $45^{\circ}$ to measure $B_{y}$ component, and finally on its side with the $x$-axis parallel to $\boldsymbol{B}_{0}$.

\subsection{Results}

\subsubsection{In vivo results}

Figure 8.3 shows the NMR magnitude images acquired with no current and the axis of orientation in relation to the main magnetic field. Figure 8.3(a) shows the images before registration and figure 8.3(b) shows the images after registration.

The images are divided into three sets (or columns in the figure), each set
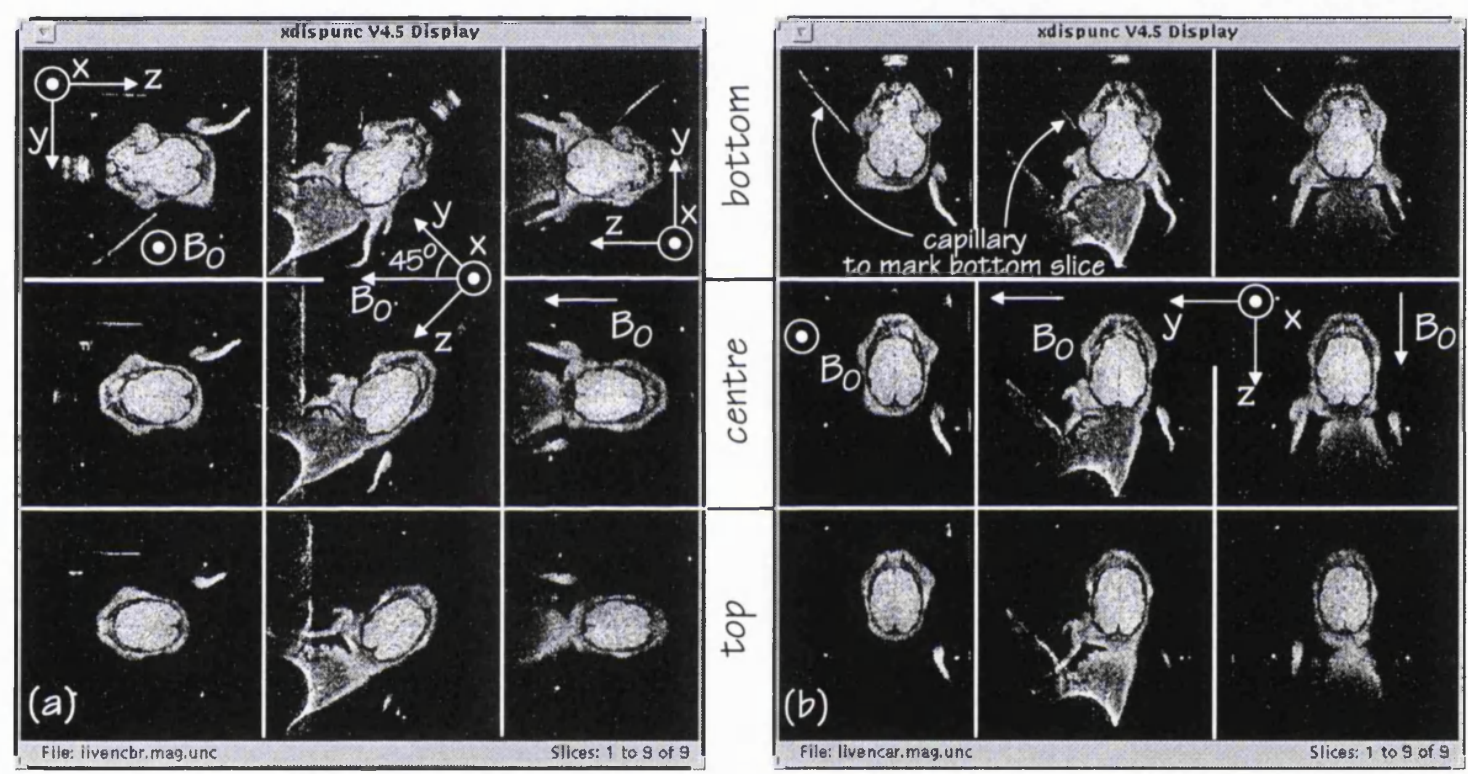

Figure 8.3 - NMR magnitude images of the "in vivo" experiment. (a) before image registration and (b) after image registration.

corresponds to one head orientation in relation to the main field and each set is composed of three different slice positions called bottom, centre and top slices (rows in the figure). All tomographic planes are parallel to the $y z$-plane. For the first two sets of images, the piglet lay on its stomach in the magnet bore and the $z$-axis was defined along the head with the frame position shown in figure 8.2. The piglet orientation with respect to the main field during this experiment was not as described in the last chapter for the whole body in vitro experiment. In this study with the piglet lying prone coronal slices were use to acquire the images with the $z$-axis parallel to $\boldsymbol{B}_{0}$, (right column in 
figure 8.3(a) and (b)), and with head inclined at $45^{\circ}$ in relation to the main field $\boldsymbol{B}_{0}$, (centre column in figure 8.3(a) and(b)). This procedure reduced the possibility of slice mis-position because the piglet body did not required repositioning and only the head was inclined in relation to the field. Also, with this procedure the NMR slice orientation and position did not need to be changed. To acquire the final images with the $x$-axis parallel to $\boldsymbol{B}_{0}$, the piglet was turned so that it lay on its side and the neck was angled forward until it was at $90^{\circ}$ in relation to the main field. The body was also slightly inclined in relation to $\boldsymbol{B}_{0}$, to minimise the angle between the head and the body.

\subsubsection{The net magnetisation degeneration}

During the experiment, when the piglet head was placed with the $x$-axis parallel to the main field, the NMR signal intensity near the neck was observed to be low. Figure 8.4 shows the transverse NMR images acquired in these circumstances with
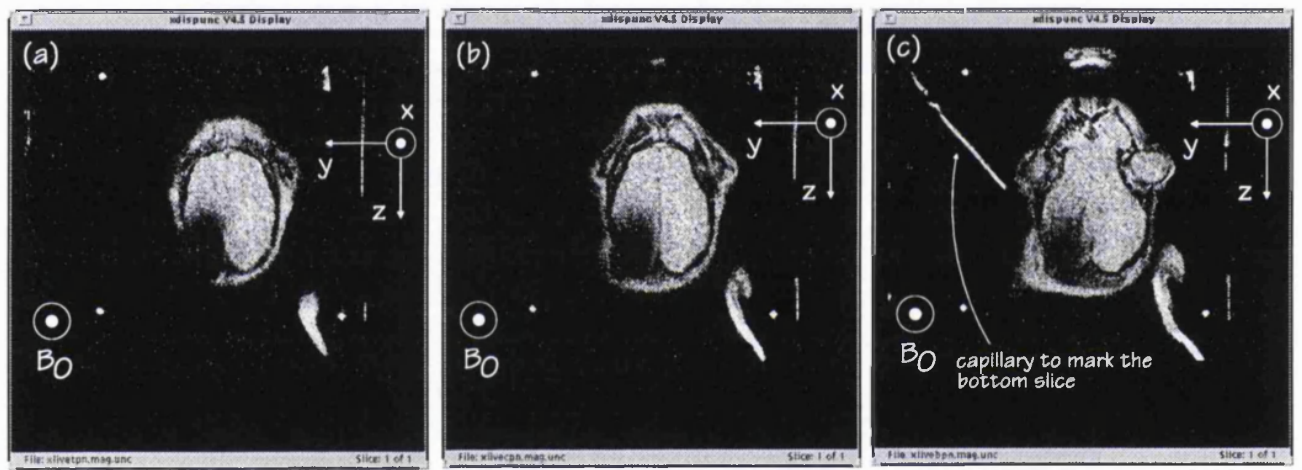

Figure 8.4-The NMR images acquired with the $x$-axis parallel to $\boldsymbol{B}_{\theta} .(a)$ top $(b)$ centre and (c)bottom slices.

electric current on. The reason for this loss in signal could not be determined at the time the animal was alive. However, after the piglet was killed and removed from the magnet bore a possible explanation was found.

The carbon cable connecting the electrode on the top of the head to the upper conductor was found to be lying near to the surface of the head. When the piglet was placed with the $x$-axis parallel to the main field, the induced magnetic field produced by the current circulating in this cable was in the same direction as $\boldsymbol{B}_{0}$, and perpendicular to the tomographic plane. The field from this cable probably produced a large inhomogeneity in $\boldsymbol{B}_{\boldsymbol{0}}$ and hence the net magnetisation in this region had completely decayed after the $80 \mathrm{~ms}$ echo-time. This effect was most marked in the top slice as shown in figure $8.4(\mathrm{a})$.

Although this problem was very much reduced when the cable was re-positioned 
to align with the main field, a small area of the image was still affected near the position where the carbon cable was bent to reach the electrode, figure 8.9 (top row). Even if we had realised before the animal was killed that this curved cable was the reason for the signal deterioration, it would have been necessary to repeat the previous image acquisitions to keep the current paths in the same position during the whole experiment. It would not have been possible to keep the animal anaesthetised for the additional period required for these studies.

\subsubsection{Electrical current magnetic field}

Figure 8.5, upper row, shows the NMR phase images of the top, centre and bottom slices acquired with the $x$-axis parallel to $\boldsymbol{B}_{0}$ and figure 8.5 , lower row, shows
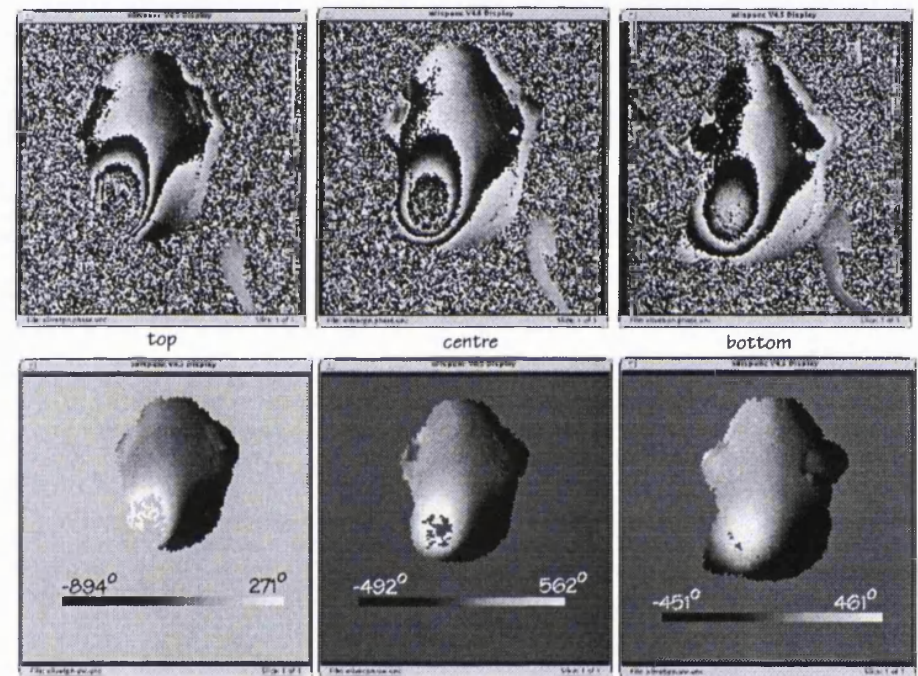

Figure 8.5 - NMR phase images acquired with the $x$-axis parallel to $\boldsymbol{B}_{0}$ to show the effect of the electrical current. (a) wrapped phase and (b) unwrapped phase.

the corresponding unwrapped phase images.

In part of the wrapped image the phase changes exceeded $180^{\circ}$ per pixel (mainly in the top slice), which suggest a very high magnetic field in the $\boldsymbol{B}_{0}$ direction. Because the $180^{\circ}$ phase changes happen in a space less than a pixel in size, the unwrapping routine is not able to unwrap the phase and the data in this region is lost. During the experiment we tried to halve the electrical current, which reduced the affected area but still a reasonable region of the head showed a large NMR signal decrease.

In figure 8.6 the calculated magnetic fields at the centre slice position are shown. The magnetic field in the $y$-direction is the result of the calculated field using equation (7.1). Due to the superposition of many different fields arising from the upper and lower 

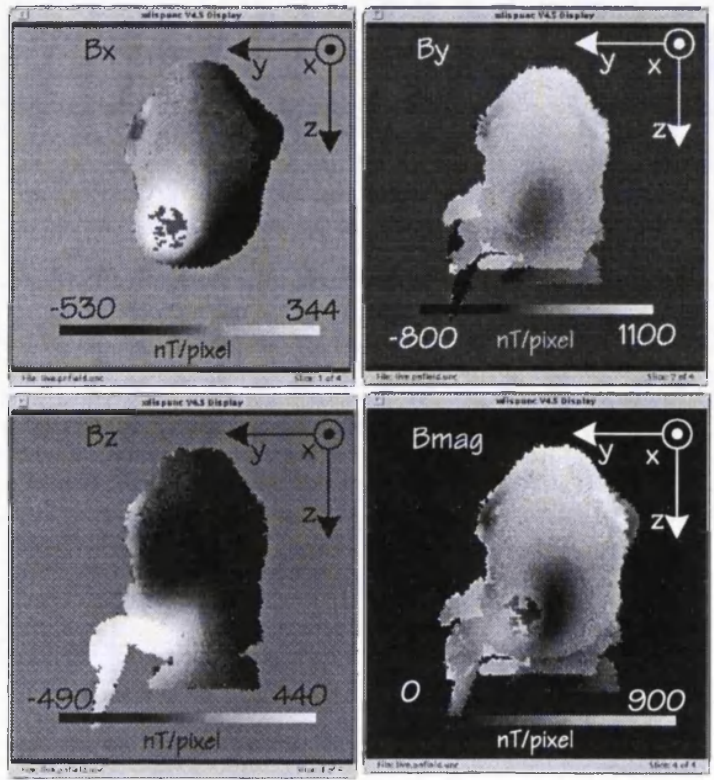

current direction applied during experiment

Figure 8.6-Electrical current magnetic field at the position of the centre slice.

conductors, the returning wire and the field produced by the carbon cable, analysis of the field using the "right hand rule" is impossible.

\subsubsection{Electrical current images}

Figure 8.7 shows the images of the electrical current component in the $x$ direction in the three adjacent tomographic planes, (a) is in the top, (b) is in the centre, and (c) is in the bottom slice. In this experiment just the positive current is shown, because only the NMR data with a positive-negative current sequence was acquired. In the figure the edges of the images show some border artifacts due to the anatomical differences between the three slices. The contrast in the three images has been stretched to a range from $-25 \mu \mathrm{A}$ to $+25 \mu \mathrm{A} / \mathrm{pixel}$.
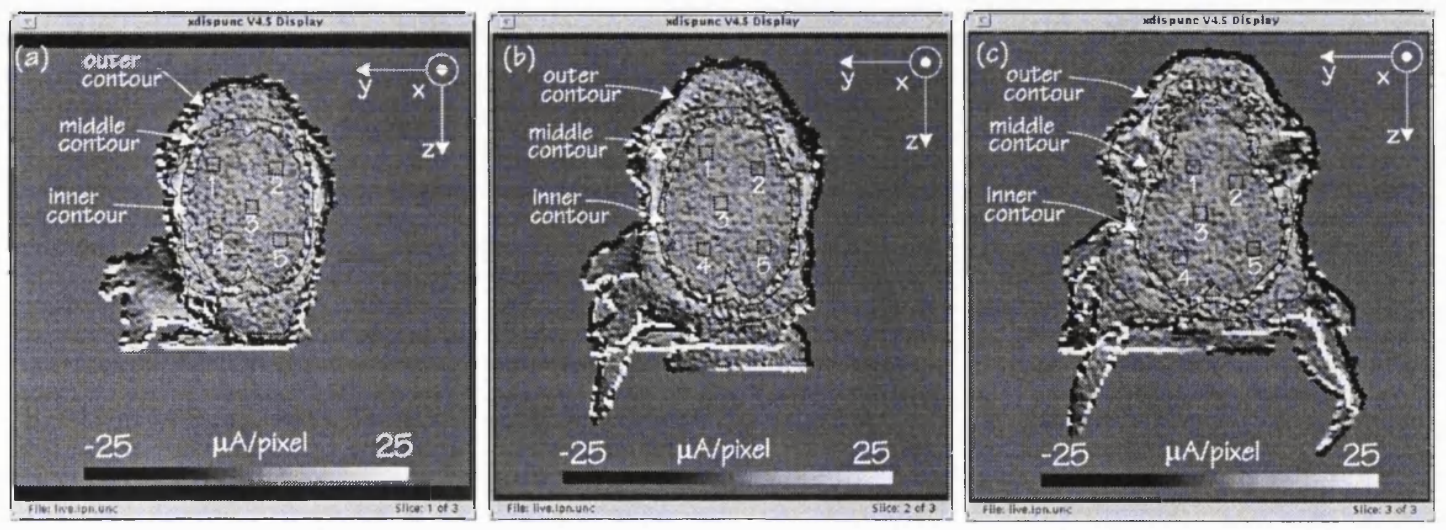

Figure $8.7-I_{x}$ current component in the top $(a)$, centre $(b)$ and bottom (c) slices. 
In figure 8.8 the electrical current components in the $y$ - and $z$-direction are shown together with the current magnitude image at the centre position of the three slices. The
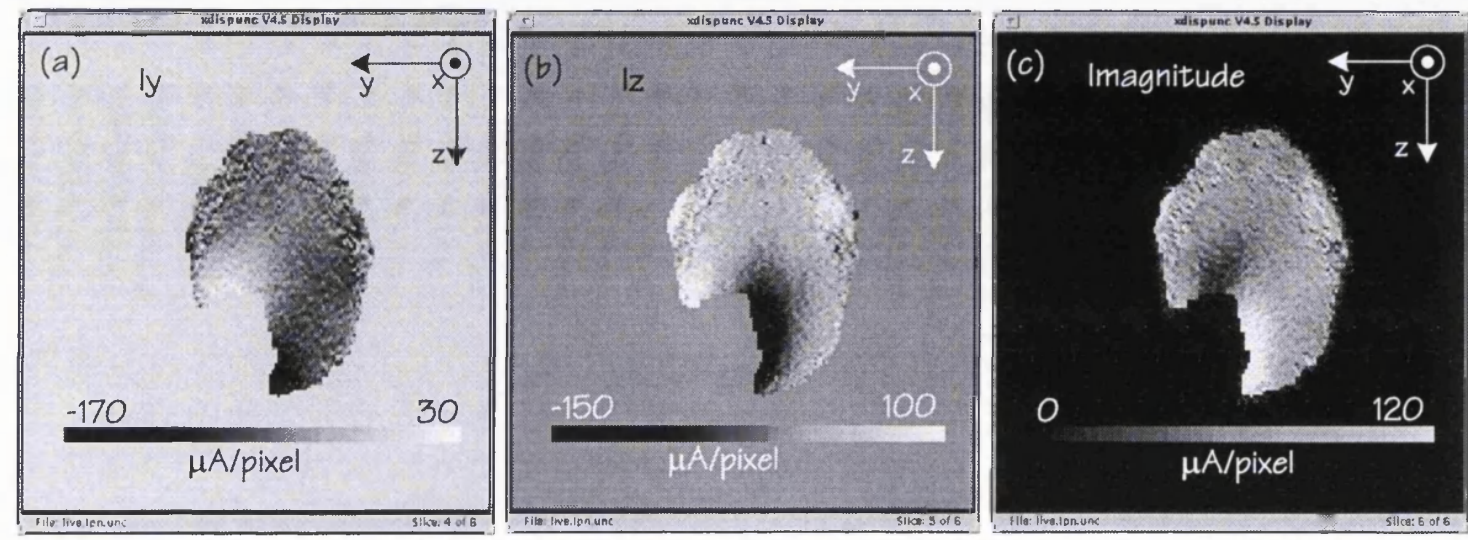

Figure $8.8-(a) I_{1},(b) I_{z}$ and magnitude current components.

edges in these images and the region where the net magnetisation was decreased were removed during the current calculation.

\subsubsection{Electrical current per region in the head}

The same procedure applied in the in vitro experiments to quantify the electrical current per region of the head was applied in this experiment. The head was divided into the three regions with the contour lines shown in the current images, figure $8.7^{20}$. Table 8.1 shows the area of each region in square millimetres, the total current integrated per region in milliamps, the percentage of the current integrated per region from the total current applied, and the ratio between the current per region and the area of the corresponding region.

The inner contour involves the brain alone, the middle contour includes the brain plus the skull and the immediately adjacent tissues, and the outer encompassed all the regions in front of the head and part of the neck. The inner and middle contours were made by the xdispunc program using the automatic region contour function. To avoid the artifact produced at the borders of the current images, the outer contour was made using the current images shown in figure 8.7. In the neck region the outer contour was drawn some millimetres apart from the middle contour. The inner and middle contours 
Table 8.1 - Results for the live animal experiment.

\begin{tabular}{|c|c|c|c|c|c|}
\hline \multicolumn{6}{|c|}{ Live experiment - Current applied $60 \mathrm{~mA}$} \\
\hline Slice & ROI & area $\left(\mathrm{mm}^{2}\right)$ & $I_{x}(m A)$ & $\%^{4}$ & $I_{x} / \operatorname{region}\left(\mu \mathrm{A} / \mathrm{mm}^{2}\right)$ \\
\hline \multirow{5}{*}{ Top } & inner $^{I}$ & 1302 & 21.92 & 36.67 & 16.84 \\
\hline & middle & 1671 & 31.99 & 53.51 & 19.14 \\
\hline & outer & 2425 & 47.76 & 79.89 & 19.69 \\
\hline & $s k u l l^{2}$ & 369 & 10.07 & 16.85 & 27.29 \\
\hline & muscle ${ }^{3}$ & 754 & 15.77 & 26.38 & 20.92 \\
\hline \multirow{5}{*}{ Centre } & inner $^{l}$ & 1484 & 22.50 & 37.64 & 15.16 \\
\hline & middle & 1867 & 28.44 & 47.57 & 15.23 \\
\hline & outer & 2978 & 49.80 & 83.31 & 16.72 \\
\hline & $s k u l l^{2}$ & 383 & 5.94 & 9.94 & 15.51 \\
\hline & muscle $e^{3}$ & 1111 & 21.36 & 35.73 & 19.23 \\
\hline \multirow{5}{*}{ Bottom } & inner ${ }^{l}$ & 1679 & 22.39 & 37.45 & 13.34 \\
\hline & middle & 2261 & 31.27 & 52.31 & 13.83 \\
\hline & outer & 3430 & 45.83 & 76.66 & 13.36 \\
\hline & $s k u l l^{2}$ & 582 & 8.88 & 14.85 & 15.26 \\
\hline & muscle ${ }^{3}$ & 1169 & 14.56 & 24.36 & 12.46 \\
\hline
\end{tabular}

1 - Brain (inner contour); ${ }^{2}$ - Brain plus Skull and adjacent tissues minus Brain (middle minus inner contour)

${ }^{3}$ - Outer minus middle contour; ${ }^{4}$ - percentage of the total current

were made using the corresponding NMR image with no current applied. The regions were saved into a file and then placed over the $I_{x}$ current images.

\subsection{Results of the experiment performed after death}

In this section the results of the experiment performed soon after the animal was killed are presented. The electrodes used were kept in the same position, i.e., in the roof of the mouth and on the top of the head and the same amount of current, $60 \mathrm{~mA}$, was applied. We also tried to keep the NMR tomographic planes at same position.

The complete experiment was performed within 4 hours after death. During the experiment the temperature of the body was monitored with the same rectal-sensor. At the beginning the temperature of the body was $37.5^{\circ}$ celsius and at the end $34.3^{\circ}$ celsius. Although the temperature of the body was reasonably maintained, the temperature of the head was probably lower than this, and would be between $34.3^{\circ}$ and the room temperature of around $25^{\circ}$ celsius. 
Figure 8.9 shows the NMR magnitude images acquired with the electric current on and after registration and the axis orientation in relation the $\boldsymbol{B}_{\boldsymbol{0}}$.

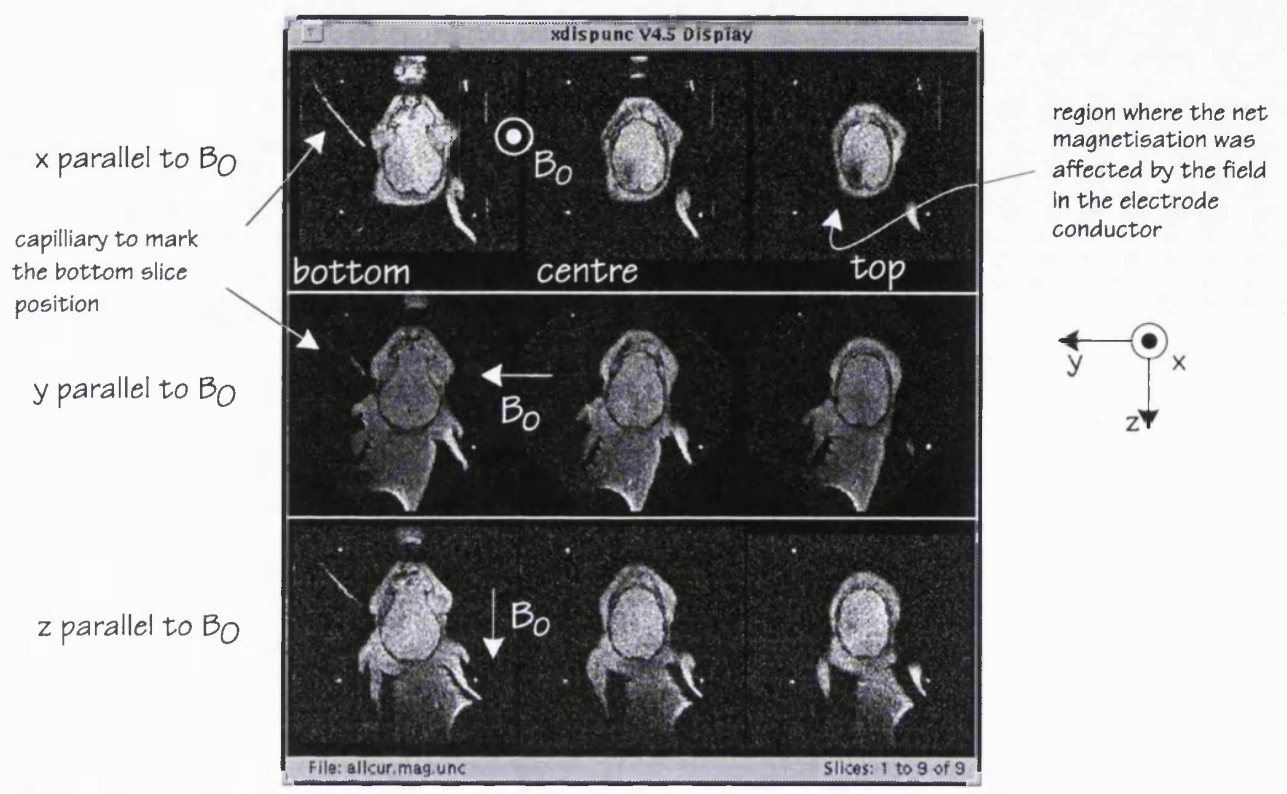

Figure 8.9-Magnitude images of the experiment performed after death

As shown in figure 8.9 , in the images with $x$-axis parallel to $\boldsymbol{B}_{\boldsymbol{0}}$, after the wire running from the electrode on the top of the head was repositioned, the NMR signal reduction was considerably reduced compared to the images shown in figure 8.4.

\subsubsection{Electrical current magnetic field}

The upper row in figure 8.10 shows the NMR phase image acquired with the $x$ axis parallel to $\boldsymbol{B}_{0}$, and the lower row shows the phase images after they were
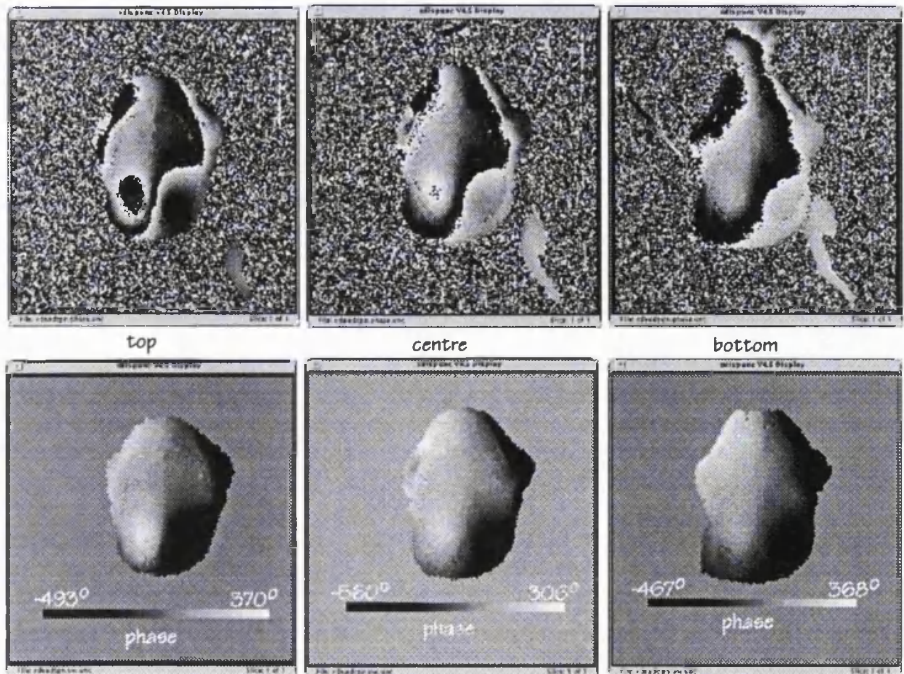

Figure 8.10 - Phase wrapped and unwrapped images acquired with the $x$-axis parallel to $B_{0}$ 
unwrapped. This figure shows that even with a very low NMR signal level in the top slice (see figure 8.9, top right) the phase image is still not affected and could be easily unwrapped.

Figure 8.11 shows the current magnetic field components $B_{x}, B_{y}$ and $B_{z}$ in the head at the centre slice position, and also the magnitude of the three components. The component in the $y$-direction, $B_{y}$, was computed using equation (7.1).
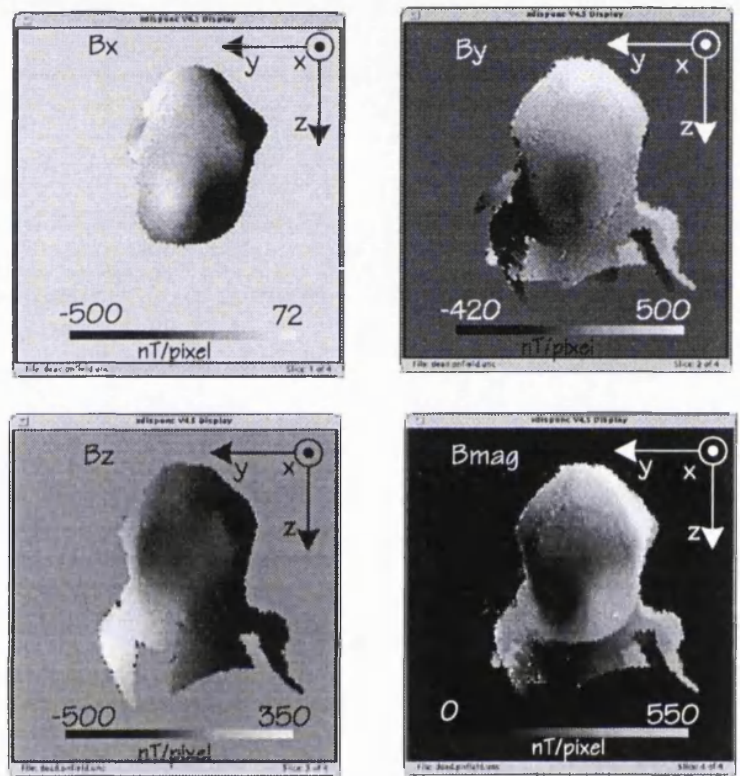

$$
\begin{aligned}
& \text { current direction } \\
& \text { applied during } \\
& \text { experiment }
\end{aligned}
$$

Figure 8.11- Electrical current magnetic field at the position of the centre slice.

\subsubsection{Electrical current images}

Figure 8.12 shows the images of the electrical current component in the $x$ direction in the three adjacent tomographic planes, (a) in the top, (b) in the centre and (c) in the bottom slice. The contrast of these three images has been stretched to a range
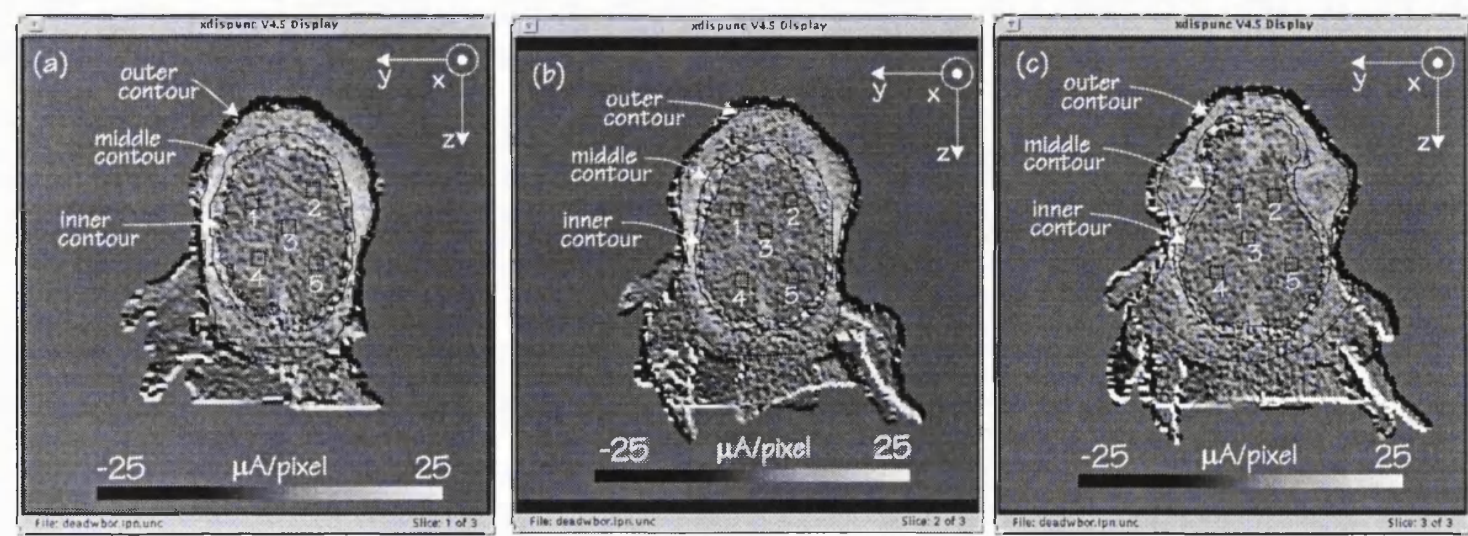

Figure $8.12-I_{x}$ current component in the after death experiment 
from $-25 \mu \mathrm{A}$ to $+25 \mu \mathrm{A} / \mathrm{pixel}$, to allow comparison with figure 8.7.

Figure 8.13 shows the current images component in the $y$ - and $z$-direction and also the current magnitude image. The edges of these images were removed during the
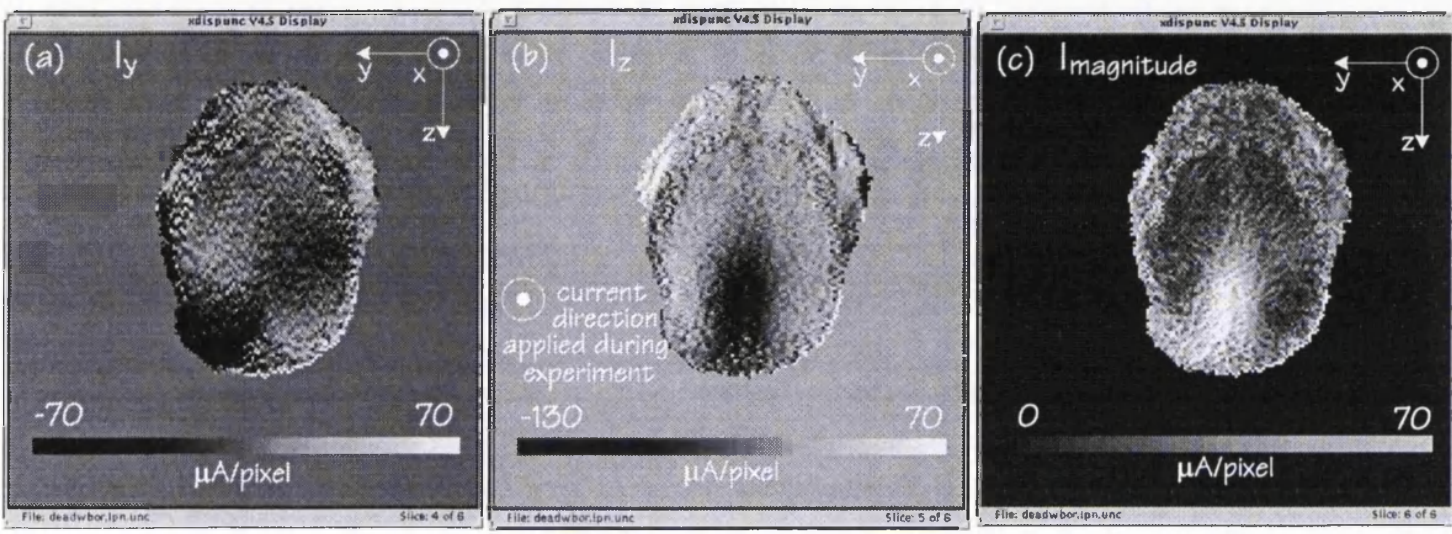

Figure 8.13-Current images (a) $I_{y}$ and (b) $I_{z}$ components, and (c) the magnitude calculated using the $I_{x}$ component in the centre slice, figure 8.12.

current calculation.

\subsubsection{Electrical current per region in the head}

As in the live animal experiment, the head was divide into 3 regions by drawing contour lines. The inner contour involves the brain, the middle encloses the brain, skull and adjacent tissues and the outer contour encompasses the whole head. These contours were made using the same strategy as before, and the results are shown in table 8.2.

\subsection{Noise analyses}

A study of the image noise similar to that performed in the in vitro experiments described in the last chapter was also performed here. Five regions were placed in the current images shown in figures 8.7 and 8.12. The results of the noise analyses in both experiments are shown in table 8.3.

In the last chapter with the in vitro experiments, it was concluded that an increase in the current-spin time integration would reduce the current standard deviation. This conclusion was not however borne out by the results in this study, since even with an increment of $20 \mathrm{~ms}$ in the time the current was applied, the experiment after death described here is noisier than any of the in vitro experiment described in the last chapter (compare tables 7.7 with table 8.3). This is because the NMR images in the experiments described here are noisier, and the only explanation found for this is that $T_{2}{ }^{*}$ may 
Table 8.2 - Results for the dead animal experiment.

\begin{tabular}{|c|c|c|c|c|c|}
\hline \multicolumn{6}{|c|}{ Dead animal experiment - Current applied $60 \mathrm{~mA}$} \\
\hline Slice & ROI & area $\left(\mathrm{mm}^{2}\right)$ & $I_{x}(m A)$ & $\%^{4}$ & $I_{x} / \operatorname{region}\left(\mu \mathrm{A} / \mathrm{mm}^{2}\right)$ \\
\hline \multirow{5}{*}{ Top } & inner ${ }^{l}$ & 1341 & 13.13 & 21.89 & 9.79 \\
\hline & middle & 1745 & 23.26 & 38.77 & 13.33 \\
\hline & outer & 2543 & 47.40 & 79.00 & 18.64 \\
\hline & $s k u l l^{2}$ & 404 & 10.13 & 16.88 & 25.07 \\
\hline & muscle ${ }^{3}$ & 798 & 24.14 & 40.23 & 30.25 \\
\hline \multirow{5}{*}{ Centre } & inner ${ }^{I}$ & 1517 & 13.07 & 21.79 & 8.62 \\
\hline & middle & 1987 & 23.56 & 39.27 & 11.86 \\
\hline & outer & 3186 & 49.19 & 81.98 & 15.44 \\
\hline & skull ${ }^{2}$ & 470 & 10.49 & 17.48 & 22.32 \\
\hline & muscle $e^{3}$ & 1199 & 25.93 & 43.22 & 21.63 \\
\hline \multirow{5}{*}{ Bottom } & inner $^{l}$ & 1815 & 15.36 & 25.61 & 8.46 \\
\hline & middle & 2259 & 23.51 & 39.18 & 10.41 \\
\hline & outer & 3522 & 46.78 & 77.97 & 13.28 \\
\hline & $s k u l l^{2}$ & 444 & 8.15 & 13.58 & 18.36 \\
\hline & muscle $e^{3}$ & 1707 & 23.27 & 38.78 & 13.63 \\
\hline
\end{tabular}

I - Brain (inner contour); ${ }^{2}$ - Brain plus Skull and adjacent tissues minus Brain (middle minus inner contour);

${ }^{3}$ - Outer minus middle contour; ${ }^{4}$ - percentage of the total current.

increase after death (subsequent to this study an increase of $T_{2}{ }^{*}$ after death was confirmed by the NMR group in the Department).

\subsection{Discussion}

In this chapter two experiments with a newborn piglet have been described, one performed in vivo and another performed soon after the animal was killed. The results show that after death there is a substantial change in the current distribution in the head, which reflects a change in the tissue electrical impedance.

The first point to be considered is the total current measured within the outer contour which encloses the whole head. In the live experiment, table 8.1, the total current and area measured are equal to $47.76 \mathrm{~mA}$ and $2425 \mathrm{~mm}^{2}$ at the top slice, $49.80 \mathrm{~mA}$ and $2978 \mathrm{~mm}^{2}$ at the centre slice, and $45.83 \mathrm{~mA}$ and $3430 \mathrm{~mm}^{2}$ at the bottom slice. In the experiment performed after death, table 8.2 , the corresponding values are $47.40 \mathrm{~mA}$ and $2543 \mathrm{~mm}^{2}$ at the top slice, $49.19 \mathrm{~mA}$ and $3186 \mathrm{~mm}^{2}$ at the centre slice and $46.78 \mathrm{~mA}$ and 
Table 8.3 - Results of the noise analysis.

\begin{tabular}{|c|c|c|c|c|c|c|c|c|c|c|c|}
\hline & \multicolumn{6}{|c|}{ In vivo experiment } & \multicolumn{5}{|c|}{ In vitro experiment } \\
\hline slice & ROI & \multicolumn{2}{|c|}{${ }^{\prime} I \mu A$} & \multicolumn{2}{|c|}{${ }^{2} \sigma(I) \mu A$} & ${ }^{3} S N R$ & \multicolumn{2}{|c|}{${ }^{\prime} I \mu A$} & \multicolumn{2}{|c|}{${ }^{2} \sigma(I) \mu A$} & ${ }^{3} S N R$ \\
\hline \multirow{5}{*}{ top slice } & 1 & 6.41 & \multirow{5}{*}{${ }^{4} 6.37$} & 5.59 & \multirow{5}{*}{${ }^{5} 5.42$} & 5.28 & 3.24 & \multirow{5}{*}{+4.1} & 4.54 & \multirow{5}{*}{${ }^{5} 3.76$} & 5.20 \\
\hline & 2 & 5.76 & & 3.99 & & 7.25 & 3.31 & & 3.01 & & 6.16 \\
\hline & 3 & 8.48 & & 5.14 & & 7.08 & 4.56 & & 3.65 & & 5.27 \\
\hline & 4 & 5.21 & & 7.57 & & 6.06 & 5.52 & & 3.65 & & 6.80 \\
\hline & 5 & 6.01 & & 4.01 & & 6.09 & 3.87 & & 3.78 & & 5.06 \\
\hline \multirow{5}{*}{$\begin{array}{c}\text { centre } \\
\text { slice }\end{array}$} & 1 & 6.76 & \multirow{5}{*}{${ }^{4} 5.41$} & 4.18 & \multirow{5}{*}{$5^{5} .08$} & 7.30 & 2.05 & \multirow{5}{*}{${ }^{4} 2.82$} & 4.61 & \multirow{5}{*}{${ }^{5} 3.71$} & 5.66 \\
\hline & 2 & 3.78 & & 3.29 & & 8.40 & 2.49 & & 3.17 & & 6.09 \\
\hline & 3 & 8.43 & & 5.18 & & 6.39 & 3.34 & & 3.87 & & 7.08 \\
\hline & 4 & 4.17 & & 4.00 & & 6.53 & 3.87 & & 3.66 & & 5.87 \\
\hline & 5 & 3.91 & & 3.47 & & 7.81 & 2.34 & & 3.00 & & 6.46 \\
\hline \multirow{5}{*}{$\begin{array}{l}\text { bottom } \\
\text { slice }\end{array}$} & 1 & 2.80 & \multirow{5}{*}{4.63} & 4.70 & \multirow{5}{*}{54.22} & 3.35 & 2.84 & \multirow{5}{*}{${ }^{4} 2.70$} & 2.45 & \multirow{5}{*}{${ }^{5} 2.85$} & 5.24 \\
\hline & 2 & 5.03 & & 3.38 & & 2.42 & 1.07 & & 2.27 & & 7.68 \\
\hline & 3 & 6.81 & & 4.97 & & 3.09 & 4.51 & & 2.93 & & 6.13 \\
\hline & 4 & 5.32 & & 2.98 & & 2.29 & 2.19 & & 3.38 & & 6.26 \\
\hline & 5 & 3.20 & & 4.69 & & 2.50 & 2.90 & & 3.08 & & 7.87 \\
\hline
\end{tabular}

${ }^{\prime} R O I$ - current average; ${ }^{2} R O I$ - current standard deviation; ${ }^{3} R O I-N M R$ magnitude average divided by the standard deviation; ${ }^{4}$ average of the five values; ${ }^{5}$ average value $=\left[\left(\sum x^{2}\right) / 3\right]^{1 / 2}$

$3522 \mathrm{~mm}^{2}$ at the bottom slice. The total area varies by approximately $1000 \mathrm{~mm}^{2}$ from the top to the bottom slice positions, but the total current is basically the same, with an average for both experiments equal to $47.8 \mathrm{~mA}$. Therefore, the remaining $12.2 \mathrm{~mA}$ in both experiments probably goes through the surface of the head, which can not be measured because of the edge artifacts, or through the part of the neck near to the body, which also cannot be measured because this region is affect by the head rotation. Because the total current measured in the head is the same, a comparison can be made between these experiments. These results are summarised in figure 8.14.

The total integrated current going through the brain is on average $62 \%$ higher in the experiment performed with the piglet alive than that performed after death $(60 \%$ in the top slice, $58 \%$ in the centre and $68 \%$ in the bottom slice). The current measured in the region around the skull is approximately the same in both studies, apart from the centre slice which was probably affected by the position of the contour lines. Comparing 

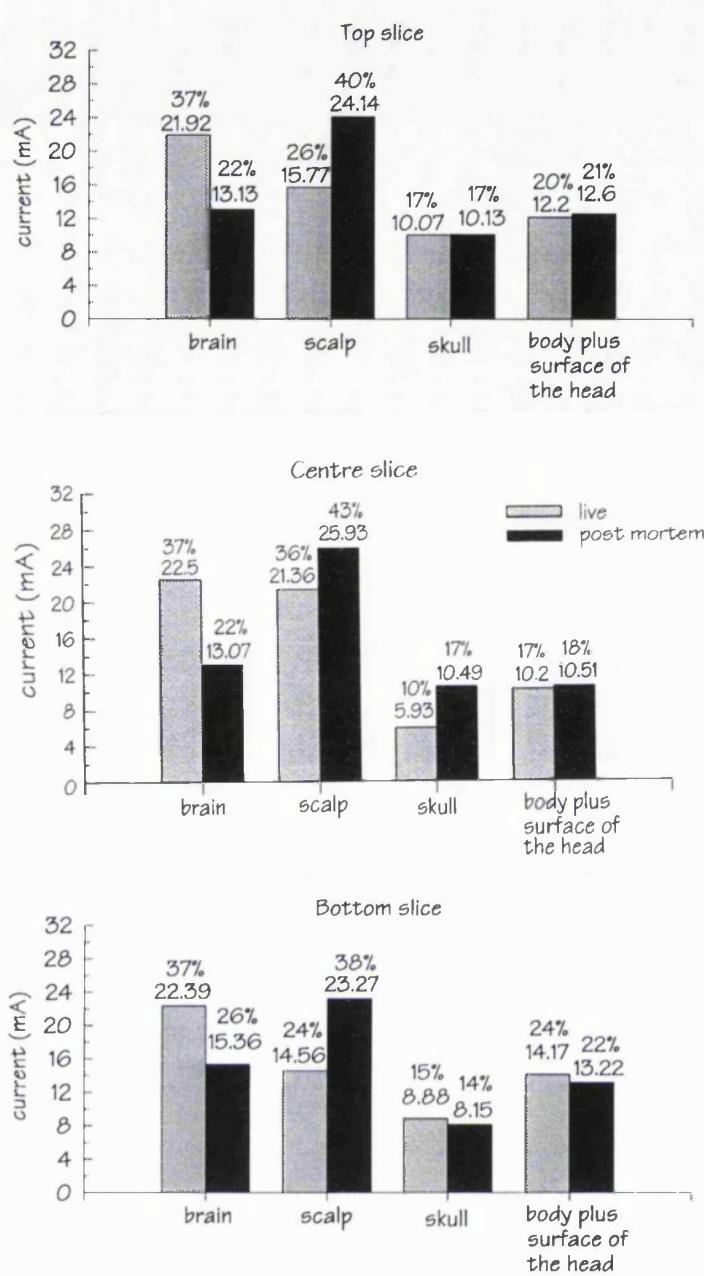

Figure 8.14 - The total current distribution in the head before and after death

the current that goes through the scalp, after death this increases by about $57 \%(53 \%$ in the top slice and $60 \%$ in the bottom slice). Because of the image artifact, the centre slice result was not considered in the average calculation. Figure 8.14 shows that the changes in current in the scalp and the brain regions are about the same magnitude but in opposite directions. Since the total current in the skull and body plus surface of the head remains constant, the reason for the current change in the head after death is associated with brain and/or scalp impedance changes.

There are several major causes of change in tissue impedance on death. Firstly there is a change in tissue blood volume as the heart stops and gravity drains the blood away. Secondly there is a change in extracellular water content as the cell ion pumps fail, and finally there is a change in temperature.

Temperature may affect the current propagation in the head. Reported values for 
the temperature coefficient of conductivity of tissues are between approximately $1 \%$ and $3 \%{ }^{\circ} \mathrm{C}^{-1}$, the highest for brain tissue being $3.2 \%{ }^{\circ} \mathrm{C}^{-1}$ (Duck, 1990). Choh-Luh et al. (1968) have measured at body temperature, $36^{\circ} \mathrm{C}$, the resistivity of the cerebral cortex as being equal to $556 \mathrm{ohm} \mathrm{cm}$ and white matter equal to $580 \mathrm{ohm} \mathrm{cm}$. At a temperature of $12^{\circ} \mathrm{C}$ they reported an increase in the cortex resistivity of $75 \%$ and in the white of $60 \%$. Van Harreveld \& Ochs(1956) observed a decrease in the conductivity of the cortex of $20 \%$ when the temperature dropped from $35^{\circ} \mathrm{C}$ to $25^{\circ} \mathrm{C}$. In our piglet study, even though the head temperature during the experiment was not monitored, the registered temperature of the piglet body at the end of the post mortem experiment was $34.3^{\circ} \mathrm{C}$. Thus it is expected that the brain temperature during the experiment should be, say, about $30^{\circ} \mathrm{C}$ (above the room temperature of $25^{\circ} \mathrm{C}$ ). Therefore, considering that during the live experiment the temperature was $37^{\circ} \mathrm{C}$, the maximum changing in the brain resistivity which could be attributed to the temperature is around $21 \%\left(\Delta \mathrm{T}\right.$ of $\left.7^{\circ} \mathrm{C}\right)$.

The effect of volume changes in the extracellular water content of the brain tissue, which is associated with cell swelling phenomena, has been studied before. Previous work using cyanide to kill the neuron cells before circulatory arrest showed that the effect of cell swelling decreases the brain conductivity by $30-35 \%$ within five minutes (van Harreveld \& Ochs, 1956). In our experiment, the animal was removed from the magnet after death, and therefore it was subsequently necessary to re-tune and re-shim the NMR machine. The first image after death was therefore acquired 10 to 15 minutes after the animal was killed, and so the effect of cell swelling should not have had any further effect on the current distribution measured during the NMR data acquisition. It is however certain that the increase in the brain tissue impedance due to cell swelling will have changed the amount of current that goes through the brain.

The depletion of blood in the head may affect the distribution of current through the brain and the scalp, although it is difficult to quantify. During the experiment, the piglet head was fixed rigidly in the frame shown in figure 8.2. This frame kept the head slightly above the level of the rest of the body, and hence, after the animal was killed, the arterial pressure would drop and the blood would tend to drain out of the head. The reported resistivity values of blood, for the dog at body temperature is equal to $144 \mathrm{ohm}$ 
centimetres and for the human $170 \mathrm{ohm}$ centimetres (Duck, 1990) ${ }^{21}$. For the brain tissue, the corresponding values are 556 and $580 \mathrm{ohm} \mathrm{cm}$ respectively for grey and white matter (Choh-Luh Li et al., 1968). Values for the scalp resistivity have not been reported but because the skin impedance is relatively high (between 10k and 10M ohms cm (Duck, 1990)) it is expected to be approximately the same as muscle, $245 \mathrm{ohm} \mathrm{cm}$ at $37{ }^{\circ} \mathrm{C}$ (Geddes and Baker, 1967). Therefore since the blood conductivity is higher, its presence should affect the current distribution in the head. However, even though the brain is much more richly perfused than the scalp or muscle (reported values for

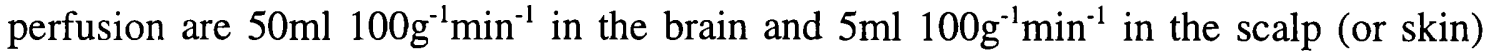
(Williams et.al., 1989)), both have approximately the same blood volume (reported values for newborn piglets are $3.33 \mathrm{ml}_{100 \mathrm{~g}^{-1}}$ in the brain and $2.19100 \mathrm{~g}^{-1}$ in the skin (Linderkamp et.al. $(1980)^{22}$ ). A value of $10 \%$ has been quoted for the increase in the brain resistivity in the absence of blood (van Harreveld \& Ochs, 1956), however although the brain and scalp contains similar blood volumes this does not necessarily imply the same increase in the scalp (no values for bloodless scalp have been reported).

It has been shown in measurements using an applied alternating current of $1 \mathrm{KHz}$ that soon after circulatory arrest caused by cutting the abdominal aorta, there is an initial, (first few minutes), rapid decrease in the brain conductivity of $30-35 \%$, following which the brain conductivity continues to slowly decrease but with a diminishing rate for the next 4-5 hours. The reasons for this decrease in the conductivity were attributed to: 1) cooling of the brain to room temperature, which accounts for a decrease of about $20 \%$ of the original conductivity, 2) drainage of fluid from the cortical surface, ventricles and perhaps from the intercellular compartment which causes a decrease of $15-20 \%$ of the original conductivity and 3) emptying of blood vessels which contributes to a decrease of $10 \%$ (van Harreveld \& Ochs, 1956).

Comparing the integrated current in the brain and its corresponding cross sectional area, in the in vivo experiment, (table 8.1), the top slice gives $21.92 \mathrm{~mA}$ and $1302 \mathrm{~mm}^{2}$, the centre slice $22.50 \mathrm{~mA}$ and $1484 \mathrm{~mm}^{2}$ and the bottom slice $22.39 \mathrm{~mA}$ and constants depending on the type of blood. 


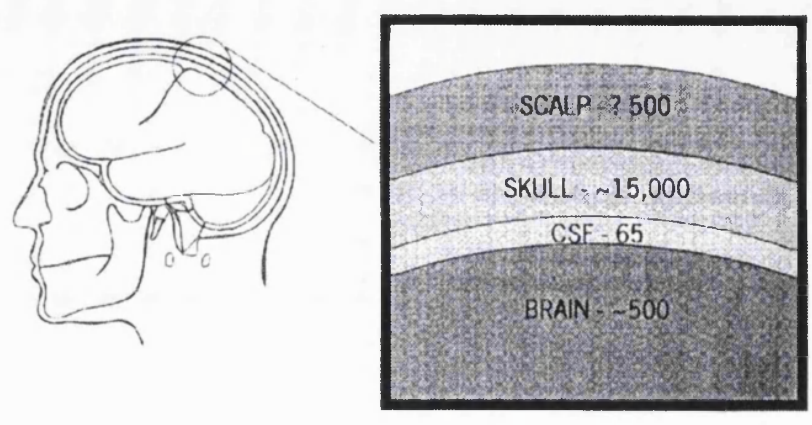

Figure 8.15 - Diagram of the estimated resistivity of the brain and extracerebral layers. Values given are in ohm centimetres (from Holder, 1993b).

$1679 \mathrm{~mm}^{2}$. The total current in each slice is practically the same, although the area of the brain has increased by $22 \%$. In the experiment performed after death, (table 8.2 ), the top slice gives $13.13 \mathrm{~mA}$ and $1341 \mathrm{~mm}^{2}$, the centre slice $13.07 \mathrm{~mA}$ and $1517 \mathrm{~mm}^{2}$ and the bottom slice $15.36 \mathrm{~mA}$ and $1815 \mathrm{~mm}^{2}$. Again the total current in each slice is practically the same but the brain area increased by $26 \%$ from the top to the bottom slice. These results suggest a constant integrated current irrespective of the brain cross section. This result can be understood with the aid of the diagram in figure 8.15. This shows that the brain is surrounded by a layer of $\mathrm{CSF}^{23}$, which has a low resistivity, about 65 ohms $\mathrm{cm}$, followed by the skull which has a higher resistivity, about $65,000 \mathrm{ohms} \mathrm{cm}$, and
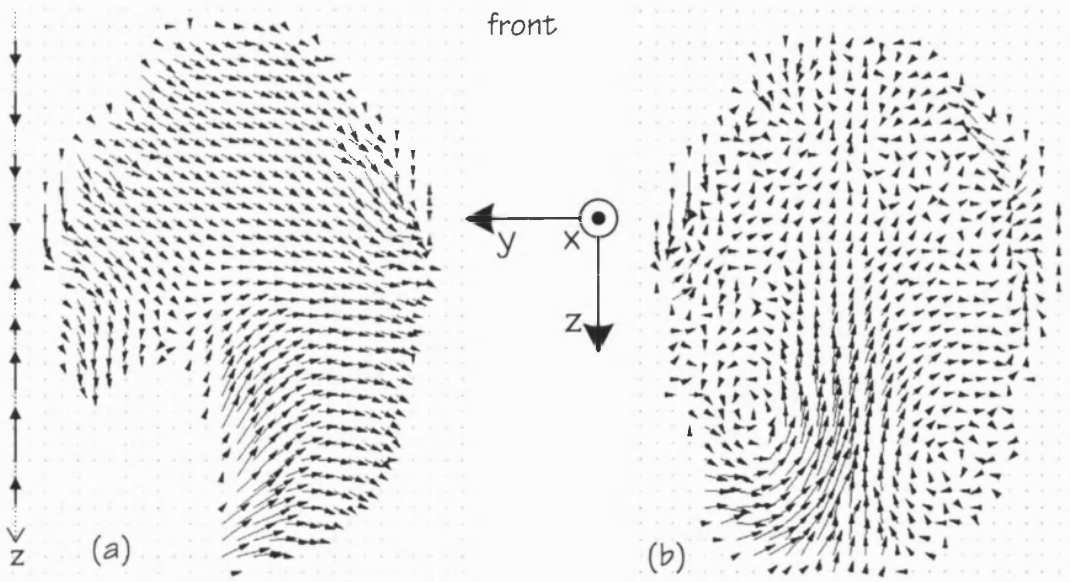

Figure 8.16 - Current vector plot (a) for the "in vivo" experiment (b) for the "in vitro" experiment. the same brightness and therefore cannot be distinguished from each other. Thus the contour line used to integrate the current in the brain also includes the current that goes through the CSF. 
finally by the scalp which has approximately the same resistivity as the brain (Holder, 1993b). Therefore, the skull would tend to act as an insulator, keeping any current that enters the skull cavity within the brain. (There must of course be some route for the observed current in the brain to enter and leave the skull cavity, and this is discussed below.)

The current orientation within the slice for both the in vivo and in vitro experiments is shown in figure 8.16(a) and (b), respectively. These vector plots were made using the current images in the $y$ - and $z$-directions. To plot the vectors, the number of pixels in the ROI was reduced with a 3 by 3 mask to average the signal intensity. These vector plots show a remarkable difference in the orientation of the current in each pixel between the live and dead animal experiments.

In figure 8.16(a) the current per pixel is mainly oriented towards one side of the head, while after death, figure $8.16(\mathrm{~b})$, this orientation disappears. A possible explanation for this is again the presence of blood in the brain. During the part of the experiment to measure the $x$-component of the current induced magnetic field, the piglet was placed on its side. This procedure means that one side of the head (the right side) is higher than the other which could give rise to a shift in the volume of venous blood towards the lower side of the head and hence increase the conductivity of this region. This explanation, or some other blood flow/volume related effect is thought to be most likely, since both the live and the dead studies required the head to be placed on its side, yet only the results from the live piglet show this asymmetry in the vector plots. It is also important to consider that if this explanation is correct, then the current distribution must have been different in the head when the piglet was placed on its side to measure the magnetic field in the $x$-direction, which would result in errors in the vector plot.

To try and confirm the theory about a blood volume shift, we measured the volume of blood shift when the head is turned to one side in an adult human volunteer using near infrared spectroscopy ${ }^{24}$. The volunteer lay supine to minimise any blood

24 The Hamamatsu NIRO 500 used in this experiment applies light at four wavelengths in the near infrared region to interrogate tissue chromophores, such as oxyhaemoglobin $\left(\mathrm{HbO}_{2}\right)$, deoxyhaemoglogin $(\mathrm{Hb})$ and oxidised cytochrome oxidase $(\mathrm{CtOx})$. Because of the unknown attenuation due to scattering in the tissue, it is not possible to measure the absolute concentration of the tissue chromophores. However since the scattering properties do not change during the measurement period, it is possible to quantitate the changes in the tissue chromophore concentrations from the measured changes in the attenuation. The $>$ 
pressure changes. The transmitting and receiving fibres were placed $4.2 \mathrm{~cm}$ apart on the left side of the head away from the midline and the temporal region. A base-line for the NIRS signal was initially recorded with the head relaxed and facing upwards (centre position). The head was then rotated through $90^{\circ}$ to the left side and the signal recorded for approximately 40 seconds. After this the head was brought back to the centre position to repeat the signal base-line acquisition. The head rotation was performed very carefully to avoid any movement of the probes with respect to the head, and the back of the head was supported so that the subject made minimal physical effort in rotating
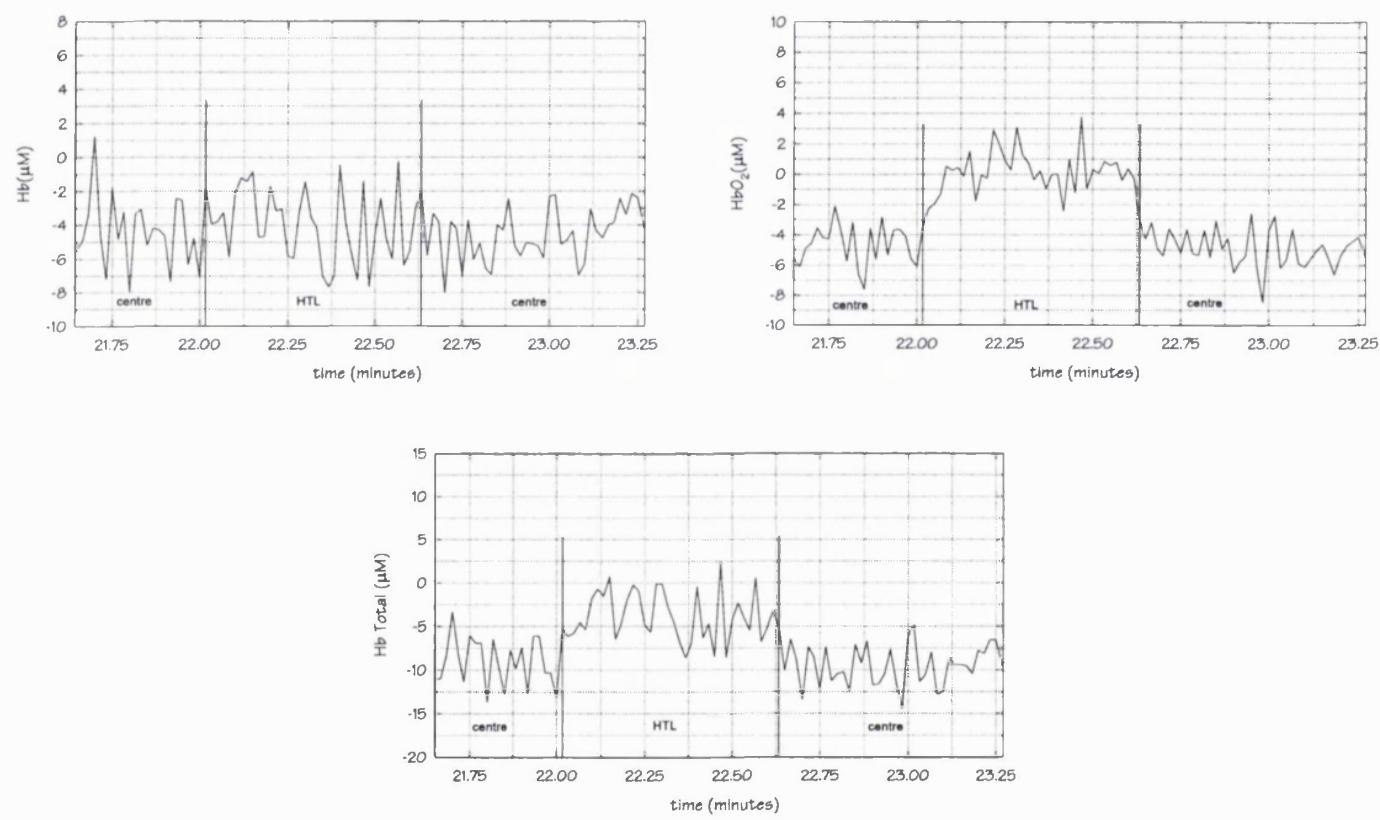

Figure 8.17 - Changes in deoxyhaemoglobin $(\mathrm{Hb})$, oxyhaemoglobin $\left(\mathrm{HbO}_{2}\right)$ and the total haemoglobin when the head is turned from the centre position to the left side.

the head. The head was then turned $90^{\circ}$ in the opposite direction for a further 40 second acquisition before returning to the centre again. This procedure was repeated three times to the left and three times to the right.

Figure 8.17 shows the changes in oxyhaemoglobin $\left(\mathrm{HbO}_{2}\right)$, deoxyhaemoglobin $\mathrm{Hb}$ and total haemoglobin $\left(\mathrm{Hb}_{\text {total }}\right)$ concentration obtained from the NIRS signal. This

sum of the $\mathrm{Hb}$ and $\mathrm{HbO}_{2}$ concentration changes provides a measure of the change in the total haemoglobin (Hb Total) (Elwell, 1995a). 

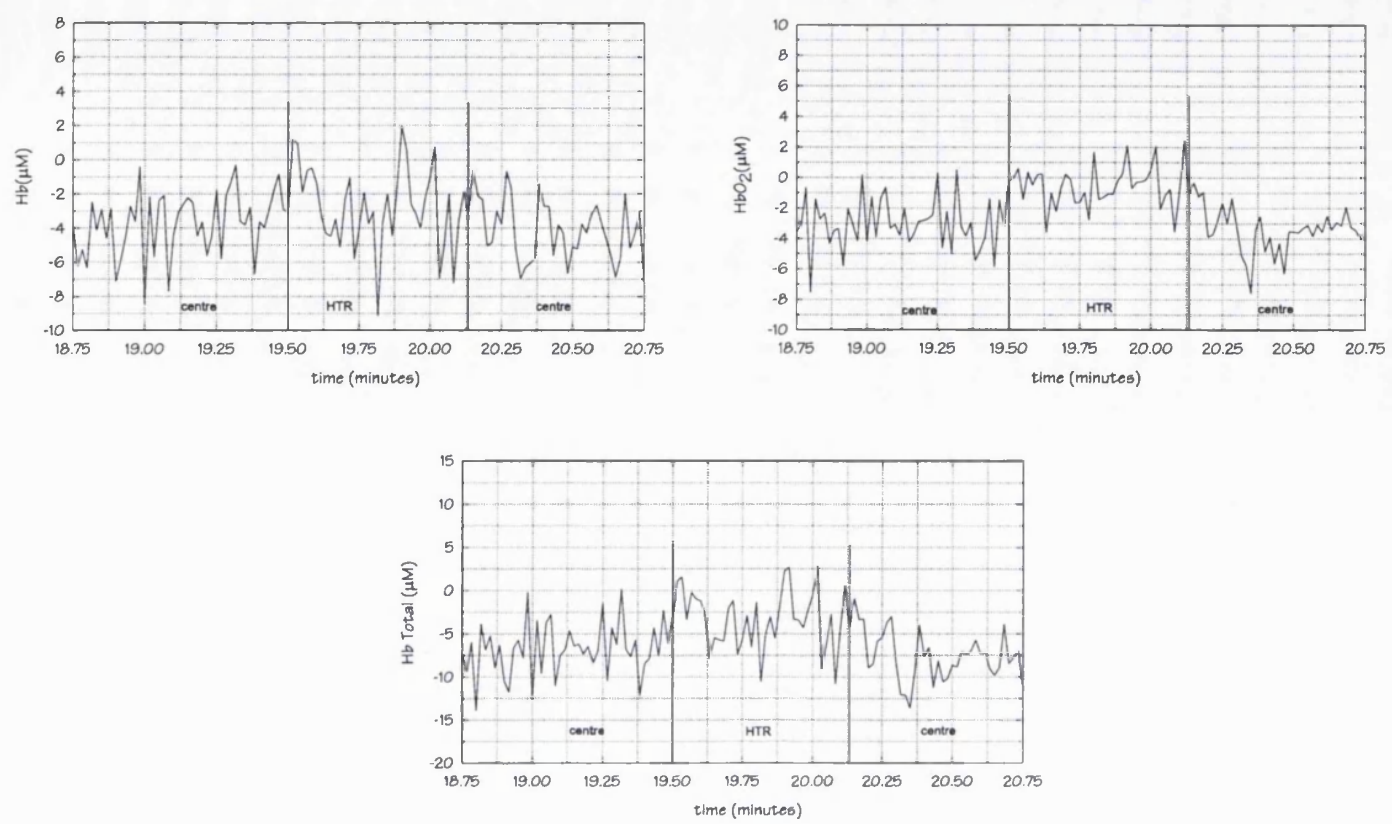

Figure 8.18 - Changes in deoxyhaemoglobin $(\mathrm{Hb})$, oxyhemoglobin $\left(\mathrm{HbO}_{2}\right)$ and the total changes in these compounds concentration when the head is tipped from the centre position to the right side.

figure demonstrates the signal recorded when the head was turned to the left. Figure 8.18 shows the equivalent changes when the head was turned to the right.

It is possible to see that the head movement does affect the blood volume distribution in the brain. For instance, figure 8.17 indicates that when the head was turned to the left side there was an increase in $\mathrm{HbO}_{2}$ concentration, beneath the probes on the lower side of the head of approximately $5 \mu$ molar. There was not a significant change in the $\mathrm{Hb}$ concentration, which is probably due to the fact that even the venous blood in the brain has only a low deoxyhaemoglobin concentration (typically $75 \%$ of the venous blood is $\mathrm{HbO}_{2}$ ). A similar but smaller effect, can be seen in figure 8.18, which

Table 8.4 - Changes in chromophore concentrations in the brain when the head is turned to the left or to the right

\begin{tabular}{|c|c|c||}
\hline & $\begin{array}{c}\text { head to the } \\
\text { left }(\mu \text { molar })\end{array}$ & $\begin{array}{c}\text { head to the } \\
\text { right }(\mu \text { molar })\end{array}$ \\
\hline$\Delta H b \mathrm{O}_{2}$ & $3.79 \pm 1.99$ & $2.73 \pm 2.04$ \\
\hline$\Delta H b$ & $0.41 \pm 2.80$ & $1.11 \pm 2.73$ \\
\hline$\Delta H b$ Total & $3.99 \pm 3.83$ & $3.85 \pm 4.05$ \\
\hline
\end{tabular}


shows the changes in the concentration when the head is rotated to the right side and the probe is now over the upper side of the brain. Here the $\mathrm{HbO}_{2}$ increases by about $2 \mu$ molar from the base line.

During the NIRS experiment the signal baseline may fluctuate, affecting the measurements of concentration changes. Also to make sure that the light received has penetrated significantly into the brain tissue, the transmitting and receiving optical fibres need to be well spaced from each other. This separation on the other hand reduces the amount of light that returns to the optical sensor resulting in a noisier signal. Therefore to quantify the changes in the chromophore concentrations, the mean value of the recorded NIRS signal for each position of the head was calculated. The average value of the three differences between the turned head position and the preceding base line are given in table 8.4 .

These values show that there is increase in the total $\mathrm{Hb}$ concentration compared to the central position when the head is turned either to the right or to the left although the rotation to the left gives a slightly higher change. The changes in $\mathrm{Hb}$ are clearly smaller than those of $\mathrm{HbO}_{2}$ and may be difficult to interpret given the level of noise of the signal.

Considering that the total haemoglobin concentration in the brain is around $50 \mu$ molar (Elwell, 1995b), the NIRS measurements show a change of approximately $8 \%$ in the blood volume at the side of the head. The fact that changes of this magnitude can occur may help to explain the electrical current orientation towards the lower side of the piglet head (figure 8.16) since blood is much more conductive than the brain tissue. Porter et al. (1964) found resistivity changes of about $1.5 \%$ during bilateral carotid compression in humans. It has also been reported that about $10 \%$ of current flow is expected to be intravascular (van Harreveld et al., 1961; Rank, 1963).

Some points need to be considered in interpreting the NIRS measurements. For instance, even though the volunteer was resting in a horizontal position to keep the blood pressure constant in the brain it is possible that when the neck was turned, the veins and/or arteries were squeezed producing changes in both the blood flow into and drainage of the blood out of the brain. Ideally this measurement should be performed on an inclinable table where the body is kept stationary and the table is inclined. Another point is that because the brain floats in a CSF layer, it may move slightly 

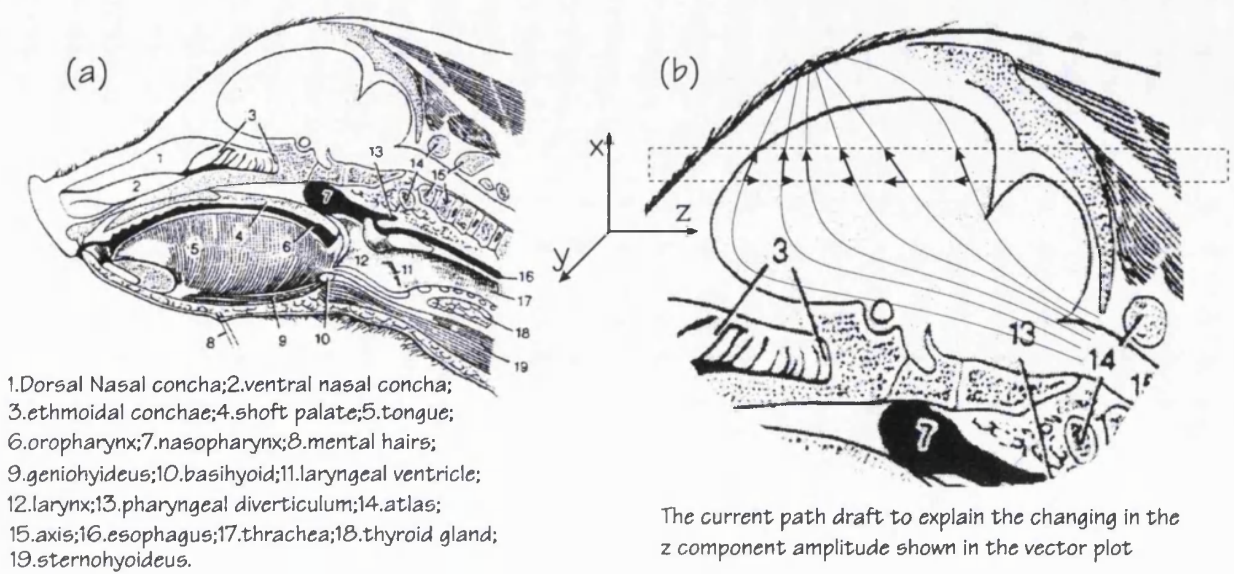

Figure 8.19 - Median section of the head of a four week old pig; the nasal septum has been removed (Sack, 1982).

towards and away from the inner skull table and hence produce a change in the NIRS signals.

One further general observation that can be made from the vector plot in figure 8.16 is that the current seems in general to be flowing into the plane from the rear of the skull. To help visualise and understand this the current propagation analyses, a drawing of a median section of the head of a four week old pig is shown in figure 8.19(a). From this figure, it can be seen that if we assume that the bones of the skull represent a high impedance path to the current flow, then with the electrode in the roof of the mouth, the only way for the current to reach the upper side of the head is through the larynx region. A current flowing through this region would produce contraction of the muscles there and close the airway, which explains the observed interference of the current with the animals breathing. It is also clear from figure 8.19(a), that the major aperture into the skull is where the spinal cord enters the brain from the spinal column. It is likely that through this aperture, the electrical current finds a path with a lower resistivity than that of going through the base of the skull. In figures 8.16 (a) and (b) the vector plots show that the current flow near to the neck is mainly oriented in the $-z-$ direction, indicating that the current comes from somewhere in the neck, otherwise it would be more equally spread as found in the in vitro experiments with the isolated head shown in figure 7.8. The vector magnitudes in figure 8.16(a) are bigger than in figure 8.16 (b) which reinforces the fact that more current reaches the brain in the in vivo experiment. In figure 8.16(a), the line on the left hand side of the vector plot 


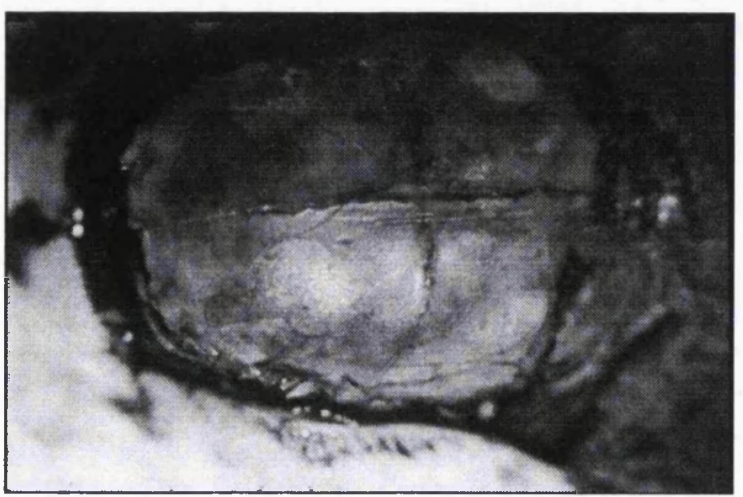

Figure 8.20 - Top view of a piglet skull

approximately shows the changes of the $z$-current component direction along the centre of the brain. In an attempt to illustrate these changes, these vectors of the current path are drawn in figure $8.19(\mathrm{~b})$, which show that at the brain edges, the $z$-component points to the centre of the brain.

A possible explanation of how the electric current leaves the skull cavity is the suture lines which join the skull bones. Figure 8.20 shows a picture of the skull of a piglet where the suture lines are easily identified. These lines intercepted at the top of the head very near to the position where the upper electrode was placed. In a newborn piglet these lines may not be completely fused and hence constitute a relatively higher conductivity path for the electrical current to reach the scalp and the upper electrode.

\subsection{Conclusion}

In this chapter the electrical current propagation in the head was studied in both the live and dead animal. The main conclusion of this experiment is that with the electrodes placed on the top of the head and in the roof of the mouth about $37 \%$ of the electrical current applied to the head reaches the brain. There was an increase of about $60 \%$ in the current that reached the brain in the live animal compared with the same experiment performed post mortem. Since the post mortem current distribution measured here was similar to that observed in previous studies on the isolated head, it appears that the distribution is not affected by positioning the electrode inside the mouth rather than on the skin surface under the neck. It was however found that inclination of the head during the live experiment affected the current distribution in the brain, possibly because of a shift in the venous blood distribution in the head. 


\section{Chapter 9}

\section{Conclusions and future work}

\section{Conclusions and future work}

In this thesis we have studied the distribution of electrical current in the head of new born piglets. The aim was to quantify the amount of current that reaches the brain through the skull and to look for new information about the distribution of the current in the head that somehow might be of use in the problem of EIT image reconstruction of the head. To measure the electrical current, a technique using NMR data was implemented. This technique is the only available method for probing the electrical current inside a medium without disturbing the electrical current propagation and the medium itself.

Since each chapter has included a discussion and conclusion of its particular contents, in this final section only the main results concerning the current measured in the head are summarised. Also discussed here are the important conclusions concerning the NMR technique itself together with comments about a new NMR RF pulse sequence that may enable the distribution of low frequency AC current to be measured.

\subsection{Results of the current measurements}

The experiments to measure the electrical current distribution in the head were performed both in vitro and in vivo. In the in vivo experiment the electrodes used to apply the electrical current were placed on the top of the head and the inside of the mouth, a post mortem experiment then followed with the electrodes in the same position. In the other in vitro experiments the electrodes were place on the top of the head and under the neck. No significant difference in the amount of current that reaches the brain was observed with the electrode inside the mouth or under the neck. The percentage current distribution per region of the head was:

- Post mortem (table 8.2)

- $\quad 23 \%$ of the total applied current passes through the brain;

- $\quad 41 \%$ of the total applied current goes through the scalp tissues;

- $\quad 16 \%$ of the total applied current goes through the skull and immediately 
adjacent tissues;

- in vivo (table 8.1)

- $\quad 37 \%$ of the total applied current goes through the brain;

- $\quad 24 \%$ of the total applied current goes through the scalp tissues;

- $\quad 28 \%$ of the total applied current goes through the skull and immediately adjacent tissues;

The current images calculated from the NMR data showed that most of the current applied onto the surface of the head, reaches the inside of the skull cavity through the aperture into the skull, where the spinal cord enters the brain. Although not yet confirmed, we assume that the electrical current leaves the brain mainly through the suture lines near to the electrode placed on the top of the head. This supposition could be verified by fastening the excised upper section of the skull across the middle of a cylinder filled up with physiological solution. To produce a uniform current inside the cylinder, the electrical current must be applied through electrodes that completely fill the ends of the cylinder. Current images could then be calculated from the NMR tomographic planes acquired as near as possible to the suture lines into the skull. This experiment should be able to disclose if there is any preferential path for the electrical current to follow across the skull barrier and will remain to be investigated in the future.

Previous work which attempted to model the neonatal head as concentric shells estimated that about $80 \%$ of the total current applied at the surface of the head passes through the brain (Rolfe et al., 1982). It is difficult to compare this modelling with the results obtained in the piglet experiments since the skull of a new born piglet head is more developed than in the human neonate with the bone sutures already formed.

A second observation from these experiments was that the current distribution in the brain is apparently affected by shifts in distribution of the blood volume and/or flow. The evidence for this can be seen in the in vivo experiment where we observed a different orientation of the electrical current in the brain compared to that in the in vitro studies. The most likely explanation for this change was thought to be a shift in the blood volume and/or flow inside the brain when the piglet was placed on its side. The assumption of blood shifts in the brain was studied in an adult volunteer using the technique of near infrared spectroscopy which showed an increase of about $8 \%$ in the volume of blood towards the lower side of the head when the head is tipped to one side. 
The fact that the majority of this increase in haemoglobin concentration was $\mathrm{HbO}_{2}$ would tend to indicate that the volume increase is associated with a rise in blood flow.

In this thesis three in vitro experiments were performed to establish the methodology to be applied in the in vivo experiment, and to verify the sensitivity and accuracy of the technique. Major practical problems relating to the method of holding the piglet head, the limited angle of rotation available during the in vivo experiment, and the method of marking the imaging slice positions were found and overcome. However, because of other remaining problems (see below) and the time limitations of an in vivo experiment, we decided to restrict the experiments to acquiring NMR tomographic planes that were always transverse to the electrode position. Ideally for EIT image reconstruction it would be better if the NMR tomographic plane matched the electrode plane. This however must remain the subject of future studies since some additional problems will certainly appear, such as the fact that near to the electrodes there will be a relatively higher current density which will produce phase changes that will exceed $180^{\circ}$ per pixel. These phase changes cannot be unwrapped and hence details of the current image near to the electrodes can not be obtained.

\subsection{The NMR technique implemented to measure electrical current}

Although the technique implemented to measure the electrical current was originally proposed by Joy et al. in 1989, so far no paper (other than abstracts) have been published about its application to the measurement of electrical current in live or dead tissues. In addition, the published sensitivity studies of this technique had so far been restricted to studies in phantoms. Therefore during the work presented in this thesis, studies with simulations, experiments performed with phantoms and also with in vitro experiments have been performed. The important features of this measurement technique can be summarised as:

- DC current

This is one of the main limitation of this technique. It requires a DC current to be applied to produce a net phase shift in the nuclear spins and because the NMR method has a relatively high noise level, a reasonable amount of current needs to be applied in the experiment. So far no one has shown AC current images at low frequency, although a method by which this may possibly be done has been suggested (see section 9.3). This remains an area for further 
research.

- Noise limitation

The images of electrical current are calculated from the NMR phase images and hence are affected by the NMR noise. Therefore the sensitivity of the technique is limited by the intrinsic NMR noise. In the noise studies (chapter 6) it was shown that the noise in the NMR phase images when the SNR ratio is higher that 3 can be considered as Gaussian with a mean value equal to zero. Since the process of current calculation from the NMR phase images is linear, the noise in the current images also has a Gaussian distribution with mean value equal to zero. Therefore, even though the determination of the current per pixel is affect by the noise it is still possible to find the amount of current per region since the mean noise value should add up zero. There is not much that can be done to reduce the noise other than signal averaging, but this would increases the total scan time. With a single scan we found that in the in vivo and in vitro experiments the standard deviation of the current noise is around $4.61 \mu \mathrm{A} / \mathrm{pixel}$ and $3.47 \mu \mathrm{A} /$ pixel (table 8.3), respectively. The lower noise value in the post mortem experiment is associated with the increase in $T_{2}{ }^{*}$ and hence NMR signal strength. To check the image contrast resolution, phantom experiments have been performed and the minimum contrast information that can be detected in the current image is approximately $17 \%$ (section 5.2).

- Object rotation and image registration

These are the main source of errors in the current measurements. Because the object needs to be rotated inside the NMR machine, it is difficult to acquire the same tomographic plane after the rotation. Therefore errors are introduced in the final current images. It is important to make the distinction that the noise in the NMR system determines the sensitivity, whilst slice mis-position and image mis-registration introduce errors into the current calculation. Errors of about $10 \%$ can be introduced by a mis-registration of about 4 pixels (equivalent to approximately $2.52 \mathrm{~mm}$ ).

In conclusion, the implemented NMR technique is able to quantify the amount of current per region in the head, but it is unable to show small changes in the current paths. For instance, the white matter in the brain contains nerve fibres which make the 
medium anisotropic, but the effects of this anisotropy in the current distribution could not be observed in the current images.

\subsection{Proposal for a new NMR technique to measure electrical current}

Ider (1995) recently proposed an NMR technique of measuring electrical current that may be able to measure AC current of low frequency. In a very simple way this technique uses the NMR technique called chemical shift imaging to measure the localised ${ }^{1} \mathrm{H}$ spectrum for each pixel position. The two-dimension FT of the $k$-space for each sample point of the spin-echo signal is a three-dimensional matrix where the third dimension is the time axes.

If during the NMR signal acquisition a time varying magnetic field is applied, the NMR signal will be modulated in frequency. From this modulation Ider claimed that the AC current could be computed. The main problem with this technique is the scan time required. For instance, if the repetition time is set to $900 \mathrm{~ms}$ (which is a reasonable value for an experiment in tissue), then for a 32 by 32 pixel image the scan time is 15.36 minutes, and for $64 \times 64$ pixels the scan time goes to 61.44 minutes. Since only the field components parallel to $\boldsymbol{B}_{\boldsymbol{b}}$ produce changes in the precession frequency this technique still requires the object to be rotated inside the NMR machine. Studies also need to be carried out to determine the maximum frequency of the AC current that can be applied in this technique. In a simulation, Ider claimed that the technique should be able to measure currents of $100 \mathrm{~Hz}$ to $1000 \mathrm{~Hz}$. Therefore this technique requires a much longer experiment and gives a lower image resolution, but offers the advantage of being able to use AC current.

We proposed here another new technique that may also be capable of measuring low frequency AC currents with the advantage of not significantly increasing the scan time. Basically the idea is to apply the $90^{\circ} \mathrm{RF}$ pulse with the slice selection gradient followed by a sequence of $180^{\circ} \mathrm{RF}$ pulses before the NMR signal is acquired. The electrical current is applied in the intervals between the $180^{\circ}$ pulses, and every time a $180^{\circ}$ pulse is applied the current direction is inverted. In this way the nuclear spins would accumulate a net phase shift, from which the electrical current can be calculated using the same procedure presented in chapter 3. Figure 9.1 illustrates the pulse sequence.

The sine wave needs to be digitised and synchronised with the NMR RF pulses. 


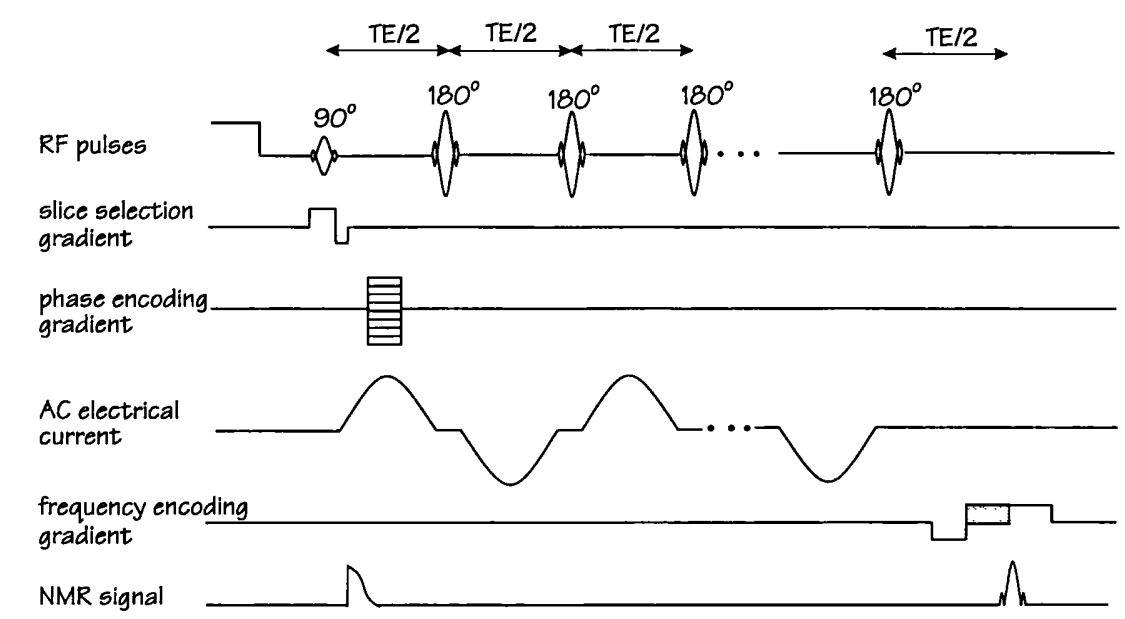

Figure 9.1 - The new RF pulse sequence to measure low frequency AC current.

The maximum frequency of this technique will be limited by how fast the $180^{\circ}$ pulses can be applied, a function of the NMR machine RF power. For instance, in the experiments described in this thesis we have used a Hermite RF pulse envelope of $3 \mathrm{~ms}$ duration and in general, the amplifier pulse gain was around 30-40, say 30 . We could reduce the pulses to $1 \mathrm{~ms}$ and increases the gain to 90 keeping the $180^{\circ}$ rotation and instead of applying a Hermite RF pulse, use a square pulse. Considering the slew-rate problem we can assume that the area of a square pulse is twice that of the Hermite shape, which means that we can halve the RF pulse to $0.5 \mathrm{~ms}$ and still have the $180^{\circ}$ spin rotation. If the RF amplifier can keep a duty cycle of $50 \%$ a frequency of $2000 \mathrm{~Hz}$ could be measured. This technique would still require the rotation of the object inside of the NMR machine.

\subsection{An additional study with the EIT tank}

We report here one final experiment (suggest by the EIT group here at UCL) that has been recently carried out to measure the current distribution inside the EIT tank described in section 5.2. For this study we removed the sponge shown in figure 5.8 and placed a cylindrical section made of plaster of Paris into the tank which produces an increase in the solution resistance of approximately 50\%. The cylinder dimensions are $70 \mathrm{~mm}$ inside diameter, $34 \mathrm{~mm}$ length and $3 \mathrm{~mm}$ wall thickness. Details of the plaster of Paris and solution used to fill up the tank are given elsewhere (Holder et al. in press). The solution also contained $\mathrm{CuSO}_{4}, 8 \mathrm{mM}$, to reduce the spin relaxation time to approximately $200 \mathrm{~ms}$. The plaster of Paris cylinder was left to equilibrate with the $\mathrm{CuSO}_{4}$ for 24 hours. Over the top and bottom of the plaster cylinder rubber "O" ring 
was used to electrically insulate the inner region of the cylinder and also to hold the annulus centrally in the EIT tank. This procedure makes sure that all the current that goes into the central region of the tank comes through the plaster cylinder wall. The measured resistivity ratio of the plaster/solution was equal to 1.56.

The current pulse amplitude was set to $24.4 \mathrm{~mA}$ and applied in the $+z$-direction of the reference axes shown in figure 5.8. The current pulses were applied for $110 \mathrm{~ms}$ and repeated every $1000 \mathrm{~ms}$. The NMR image parameters were set to: echo time $110 \mathrm{~ms}$, repetition time $1000 \mathrm{~ms}$, slice thickness $3 \mathrm{~mm}$ and image matrix 128 by 128 . The tomographic plane was set to match the plane defined by the electrodes placed around the EIT tank.

Figure 9.2 shows one of the NMR images acquired with no current to illustrate

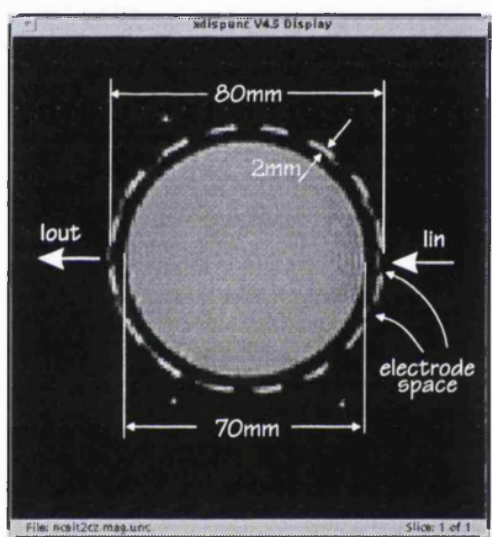

Figure 9.2 - NMR magnitude image of the EIT phantom with the plaster of Paris cylinder the different regions of the EIT tank with the Plaster of Paris annulus in place. In this figure the electrodes used to apply the electrical current can also be seen.

Figure 9.3 shows the components of electrical current in the $x$-direction, $I_{x}$, the $y$-direction, $I_{y}$, the $z$-direction, $I_{z}$ and the current magnitude, $I_{\text {mag }}$. The electrical current was computed just in the region inside of the cylinder defined by the plaster of Paris.

To compare the current distribution with and without the plaster cylinder the results shown in section 5.2 were used. Although in this experiment there is a sponge in the centre its effect on the current distribution is hardly visible as shown in figure 5.8. Figure 9.4 shows three vertical profiles at $5 \mathrm{~mm}$ (a), $22 \mathrm{~mm}$ (b) and $35 \mathrm{~mm}$ (c) from the inner edge of the plaster of Paris.

The amount of current per pixel can not be compared in these two experiments 


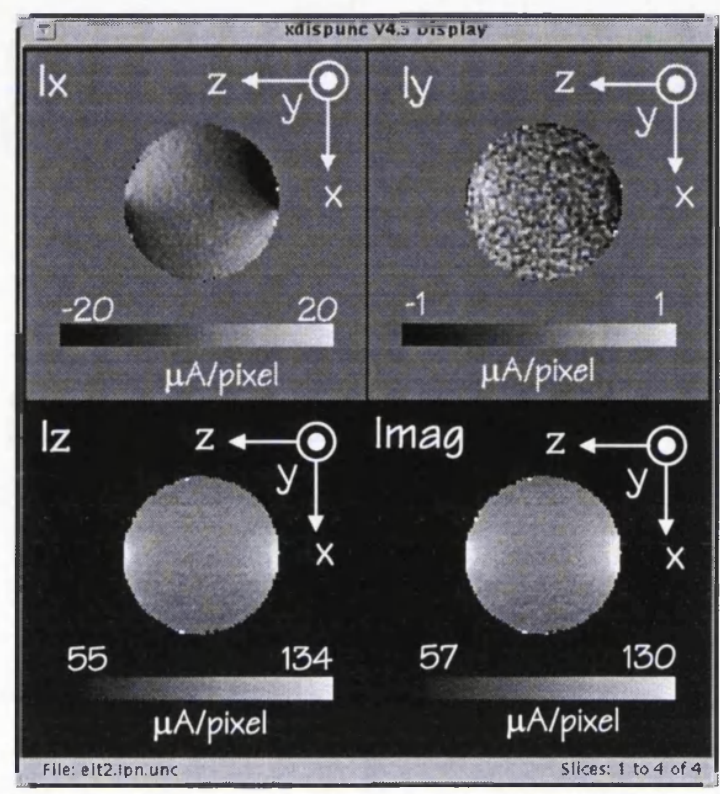

Figure 9.3 - Current components inside the plaster cylinder placed in the centre of the EIT phantom.

because different NMR image parameters and currents were used during the experiments. In the experiment with the plaster cylinder, the NMR image parameters were set to: pixel size $0.94 \mathrm{~mm}$, slice thickness $3 \mathrm{~mm}$ and image matrix 128 by 128 , and the current applied was $24.4 \mathrm{~mA}$. In the experiment described in section 5.2 the NMR parameters were set to: pixel size 0.47 , slice thickness $2 \mathrm{~mm}$ and image matrix 256 by 256 , and the current applied was $18.3 \mathrm{~mA}$. Therefore to compare the general shape of the current distribution profiles, the baseline of the experiment performed with the plaster annulus was moved to a negative value as shown in figure 9.4. These profiles show that although the plaster of Paris increases the local resistivity by about $50 \%$ the current profile in the central section is basically the same. 


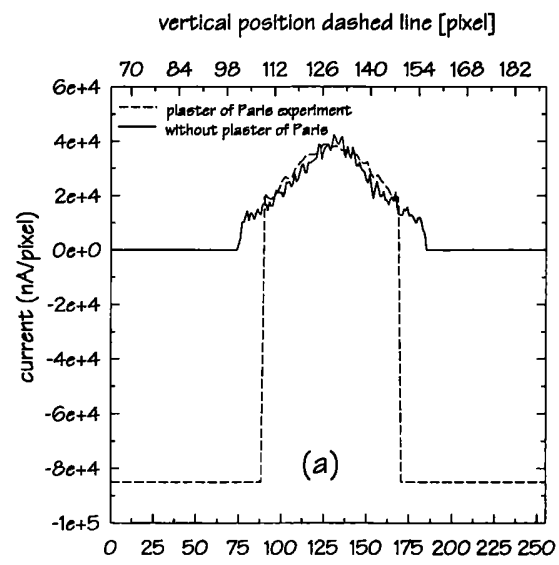

$5 \mathrm{~mm}$ from the plasticter infiner edge

vertical profile dashed line [pixel]

vertical position dashed line [plxel]
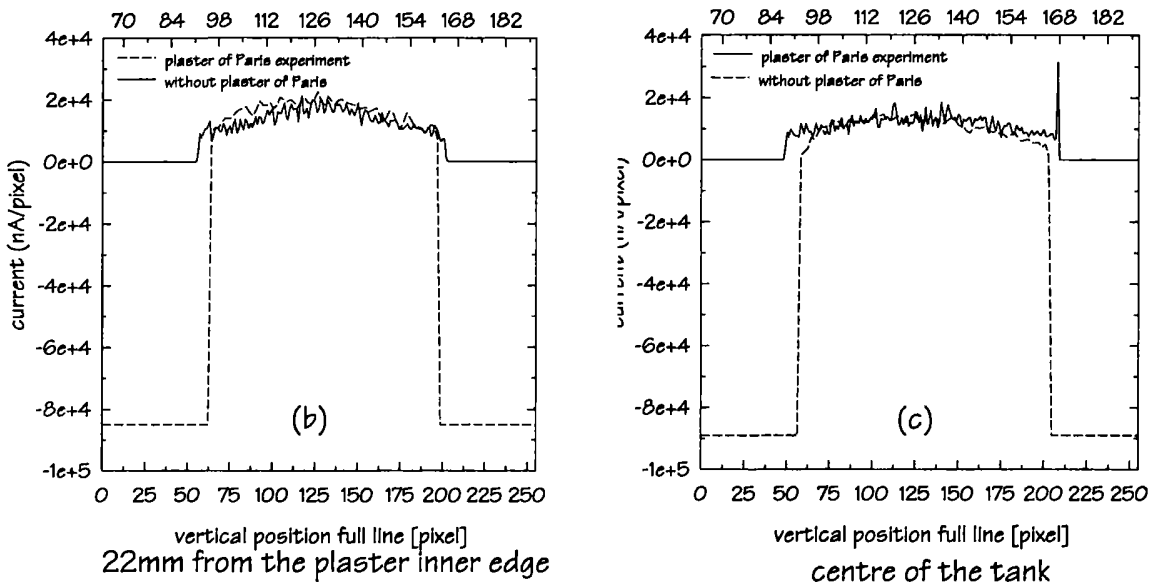

Figure 9.4 - Current profiles with and without the plaster of Paris annulus in the EIT tank. 


\section{Appendix A - Formal development for equation (2.66)}

$$
\begin{aligned}
& F_{k}=\frac{1}{N} \sum_{n=0}^{2 N-1} S_{n} e^{-i \frac{2 \pi k n}{N}} \\
& F_{k}=\frac{1}{N} \sum_{n=0}^{N-1}\{\Re(2 n)+i \mathfrak{I}(2 n+1)\} e^{-i \frac{2 \pi k n}{N}} \\
& F_{k}=\frac{1}{N} \sum_{n=0}^{N-1}\left\{\cos \left(\frac{2 \pi f n}{N}\right)+i \sin \left(\frac{2 \pi f(n+1 / 2)}{N}\right)\right\} e^{-i \frac{2 \pi k n}{N}} \\
& F_{k}=\sum_{n=0}^{N-1}\left\{\cos \left(\frac{2 \pi f n}{N}\right)+i \sin \left(\frac{2 \pi f n}{N}+\frac{\pi f}{N}\right)\right\} e^{-i \frac{2 \pi k n}{N}} \\
& F_{k}=\sum_{n=0}^{N-1}\left\{\cos \left(\frac{2 \pi f n}{N}\right)+i\left[\sin \left(\frac{2 \pi f n}{N}\right) \cos \left(\frac{\pi f}{N}\right)+\sin \left(\frac{\pi f}{N}\right) \cos \left(\frac{2 \pi f l}{N}\right)\right]\right\} e^{-i \frac{2 \pi k n}{N}}
\end{aligned}
$$

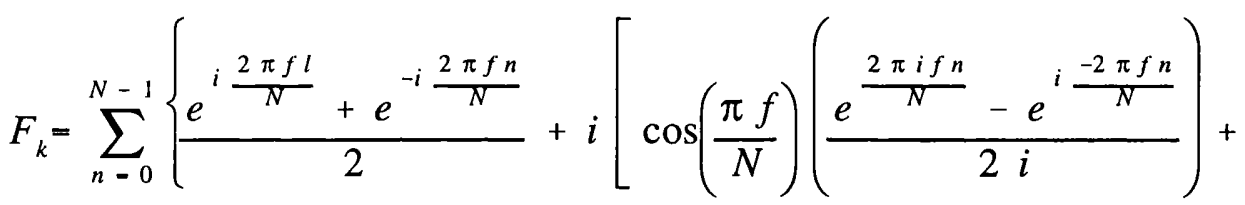

$$
\begin{aligned}
& \left.\left.\sin \left(\frac{\pi f}{N}\right)\left[\frac{e^{i \frac{2 \pi f n}{N}}+e^{-i \frac{2 \pi f n}{N}}}{2}\right)\right]\right\} e^{-i \frac{2 \pi k n}{N}} \\
& F_{k}=\sum_{n=0}^{n-1} \frac{1}{2}\left\{e^{-i \frac{2 \pi n(k-f)}{N}}+e^{-i \frac{2 \pi n(k+f)}{N}}+\cos \left(\frac{\pi f}{N}\right)\left[e^{i \frac{2 \pi n(k-f)}{N}}-e^{\left.-i \frac{2 \pi n(k+f)}{N}\right]}+\right.\right. \\
& \left.i \sin \left(\frac{\pi f}{N}\right)\left[e^{-i \frac{2 \pi n(k-f)}{N}}+e^{-i \frac{2 \pi n(k+f)}{N}}\right]\right\} \\
& F_{k}=\sum_{n=0}^{N-1}\left[1+\cos \left(\frac{\pi f}{N}\right)\right] e^{-i \frac{2 \pi n(k-f)}{N}}+\left[1-\cos \left(\frac{\pi f}{N}\right)\right] e^{-i \frac{2 \pi n(k+f)}{N}}+ \\
& i\left\{\sin \left(\frac{\pi f}{N}\right)\left[e^{-i \frac{2 \pi n(k-f)}{N}}+e^{-i \frac{2 \pi n(k+f)}{N}}\right]\right\} \\
& F_{k}=\frac{N}{2}\left\{\left[1+\cos \left(\frac{\pi f}{N}\right)\right] \delta_{(k-f)}+\left[1-\cos \left(\frac{\pi f}{N}\right)\right] \delta_{(k+f)}+i \sin \left(\frac{\pi f}{N}\right)\left[\delta_{(k-f)}+\delta_{(k+f)}\right]\right\}
\end{aligned}
$$




\section{Appendix B -Formal development for equation (2.74)}

$$
\begin{gathered}
F(k)=\sum_{n=0}^{N-1}(-1)^{n}\left[\frac{e^{i \frac{2 \pi f n}{N}}+e^{-i \frac{2 \pi f n}{N}}}{2}-\left(\frac{e^{i \frac{2 \pi f(n+1 / 2)}{N}}-e^{-i \frac{2 \pi f(n+1 / 2)}{N}}}{2}\right) e^{-i \frac{\pi k}{N}}\right] e^{-i \frac{2 \pi n k}{N}} \\
F(k)=\sum_{n=0}^{N-1} \frac{(-1)^{n}}{2}\left[e^{-i \frac{2 \pi n(k-f)}{N}}+e^{-i \frac{2 \pi n(k+f)}{N}}+i\left(e^{-i \frac{2 \pi n(k-f)}{N}} e^{-i \frac{\pi(k-f)}{N}}-e^{-i \frac{2 \pi n(k+f)}{N}} e^{\left.-i \frac{\pi(k+f)}{N}\right)}\right]\right. \\
F_{k}=\sum_{n=0}^{N-1} \frac{(-1)^{n}}{2}\left[\left(1+i e^{-i \frac{\pi(k-f)}{N}}\right) e^{-i \frac{2 \pi n(k-f)}{N}}+\left(1-i e^{\left.-i \frac{\pi(k+f)}{N}\right)} e^{-i \frac{2 \pi l(k+f)}{N}}\right]\right.
\end{gathered}
$$

substituting $i=e^{i \pi / 2}$ and $(-1)^{l}=e^{i \pi l}$,

$$
\begin{gathered}
F_{k}=\sum_{n=0}^{N-1} \frac{e^{i \pi n}}{2}\left[\left(1+e^{\frac{i \pi}{2}} e^{-i \frac{\pi(k-f)}{N}}\right) e^{-i \frac{2 \pi n(k-f)}{N}}+\left(1-e^{\frac{i \pi}{2}} e^{-i \frac{\pi(k+f)}{N}}\right) e^{-i \frac{2 \pi n(k+f)}{N}}\right] \\
F_{k}=\sum_{n=0}^{N-1} \frac{1}{2}\left(1+e^{-i \frac{\pi(k-f-N / 2)}{N}}\right) e^{-i \frac{2 \pi l(k-f-N / 2)}{N}}+\frac{1}{2}\left(1-e^{-i \frac{\pi(k+f-N / 2)}{N}}\right) e^{-i \frac{2 \pi l(k+f-N / 2)}{N}}
\end{gathered}
$$

now for $k=N / 2-f$ the second round bracket is equal to zero, where the delta function exists, and for $k=N / 2+f$ the first round brackets is equal to two. Therefore,

$$
F(k)=\frac{1}{2} 2 N \delta_{(k, N / 2+f)}=N \delta_{(k, N / 2+f)}
$$




\section{Appendix $C$}

\section{Programmable Current Source and NMR Trigger Control}

To perform the NMR experiments a fully programmable bipolar current source is necessary. The source should have adjustable current amplitude, current time (both on and off), current pulses period, and should be capable of being accurately synchronised with the NMR pulse sequence.

In addition to these general features, the physical environment of a NMR system imposes some special requirements on any electronic instrument that will be placed inside the Faraday/magnetic shield cage. Firstly, the instrument must be built in a non magnetic box (and the circuits must contain no ferromagnetic components). Secondly, it should ideally have a self contained power supply, and finally it should not introduce RF signals into the shield cage, which could compromise the NMR signal.

To fulfil all these requirements, a fully programmable current source was implemented. The block diagram of the system is shown in figure C.1. Basically the circuit is divided into two parts: 1) A self contained Current Source Unit, 2) The Current Pulse and NMR Trigger Control.

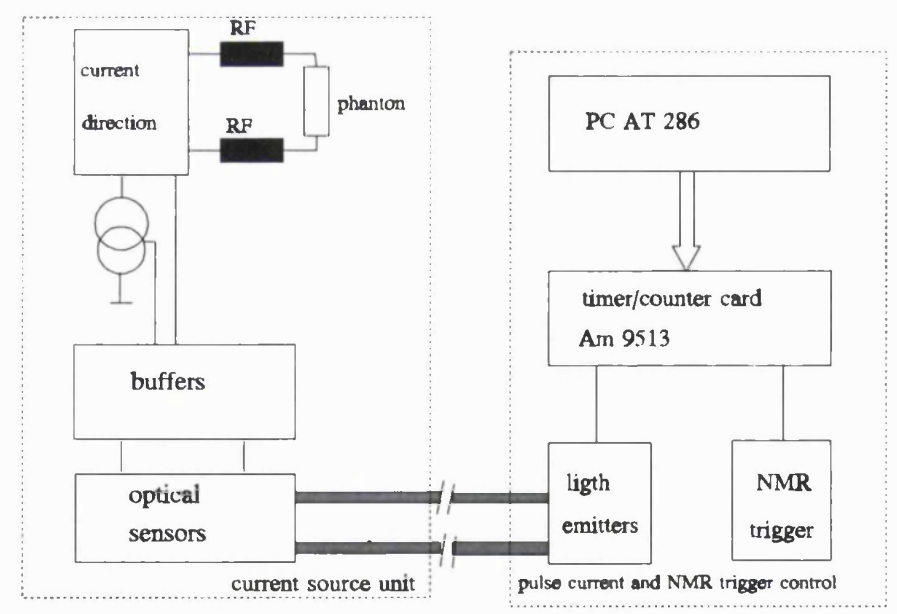

Figure C.I - Block diagram of the Current Supply and the Current Pulse and Trigger Control

The Current Source Unit is composed of a controllable constant current amplitude section, the current direction control, RF filters, two optical sensors and a set of 4 rechargeable batteries $(48 \mathrm{~V})$. The Current Pulse and NMR Trigger control is composed of a Timer/Counter card, a PC-AT286, two light emitters and the NMR trigger circuit. These units are linking by a pair of optical fibres. 


\section{C.1. The Current Source Unit}

The Current Source Unit was implemented in a diecast aluminium box and the power was supplied by a set of 4 rechargeable batteries of 12 volts each, totalling 48 volts. These features especially the use of a self contained power supply avoid any interference in the image process and permit the use of currents of up to $124 \mathrm{~mA}$.

To produce a constant controllable current, a MOSFET and an operational amplifier were used, figure C.2 shows the configuration. The operational amplifier compares the voltage on resistor $\mathrm{R}_{\mathrm{S}}$, which is proportional to the output current, and the voltage on a multi-turn potentiometer, which is used to set the desired current. The difference between these two voltages is applied at the MOSFET gate, controlling its channel resistivity and hence the output current.

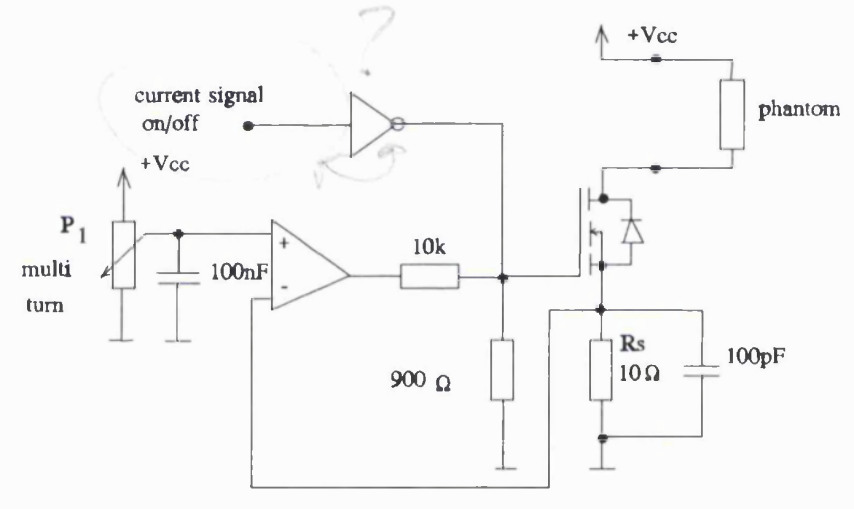

Figure C.2 - The controllable constant current supply. $R_{s}$ is used to sample the applied current. $P_{1}$ is used to control the current amplitude

The multi-turn potentiometer, $\mathrm{P}_{1}$ enables the current amplitude to be linearly controlled between 0 and $124 \mathrm{~mA}$.

To control the current direction through the phantom, four optically isolated SCR's are used (figure C.3). Opposing pairs of SCR's are switched simultaneously with a short interval when the current is turned off. This interval comprises the period during which the $180^{\circ}$ NMR pulse is applied, (the current must be switched off, to avoid any interference with the refocusing pulse). To turn the current off an open-collector buffer is used which switches the MOSFET gate to ground.

Since the NMR imaging process involves exposing the phantom to rapidly changing magnetic field gradient, it is necessary to protected the current source from high voltages which may be induced in the leads connecting it to the phantom. A pair 


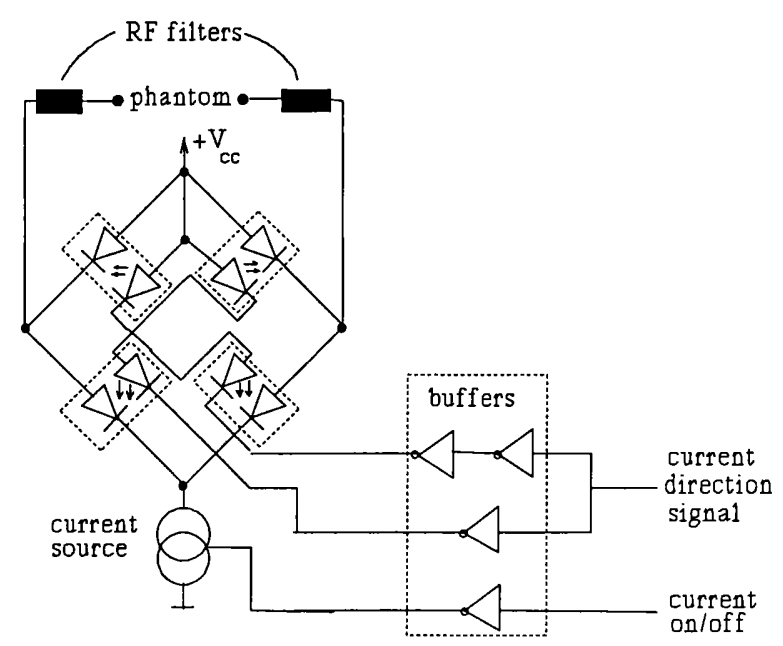

Figure C.3 - Current direction control. Four optical SCRs are used to control the current direction.

of RF filters was used for this purpose, and these also act to prevent any RF signals from the supply entering the bore of the NMR system.

\section{C.2. Current Pulse and NMR Trigger Control.}

The Current Pulse and NMR Trigger Control (figure C.1) is responsible for providing the digital signals to control the Current Supply Unit and the NMR Trigger Pulse Sequence.

All the digital signals are generated by a Fairchild Counter/Timer Card, Model PCL-830. The card is programmed by a PC-AT286. The counter timer board card uses two Advanced Micro Devices Am9513 System Timing Controllers. Each of these devices has five general-purpose 16 bit counters. Just one device was necessary for the initial phase of the project.

The Counter/Timer Board is controlled by a $\mathrm{C}$ programme which permitted control of the Repetition Time (TR) for the NMR pulse sequence, the Echo Time (TE) to fit the current pulses into the TE/2 intervals, the Current Time (TC) which is divided into equal parts for the positive and negative pulse, and the Current Interval (TD), during which the refocusing pulse is applied.

The Bruker System has a hardware trigger normally used to enable synchronous cardiac gating from an ECG monitor. To trigger the NMR pulse sequence, it is necessary to supply a negative going edge on pins 5 and 6 of the input connector for the ECG Monitor.

The trigger signal is generated by one of the counters, which is programmed in 
mode D - Rate Generate with no Hardware Gating. This mode generates a pulse sequence, whose period is set to the desired Repetition Time (TR) for the NMR pulses. The signal from Terminal Count output of this counter is interfaced with the NMR input trigger, by an open-collector invertor.

To control the current pulses and direction, another 3 counters are used. These counters are programmed in mode L - Hardware-Triggered Delayed Pulse One-Shot (Firchild Technical Manual, 1990) and the Terminal Count Toggled outputs from these counters were selected. The signal trigger for these counters is the signal from the Terminal Count output of the counter responsible for the Repetition Time. Two of these counters are used to define the period of the half Current Time, and the other one is used for the current direction. The on/off and delay times are set as shown in figure C.4.

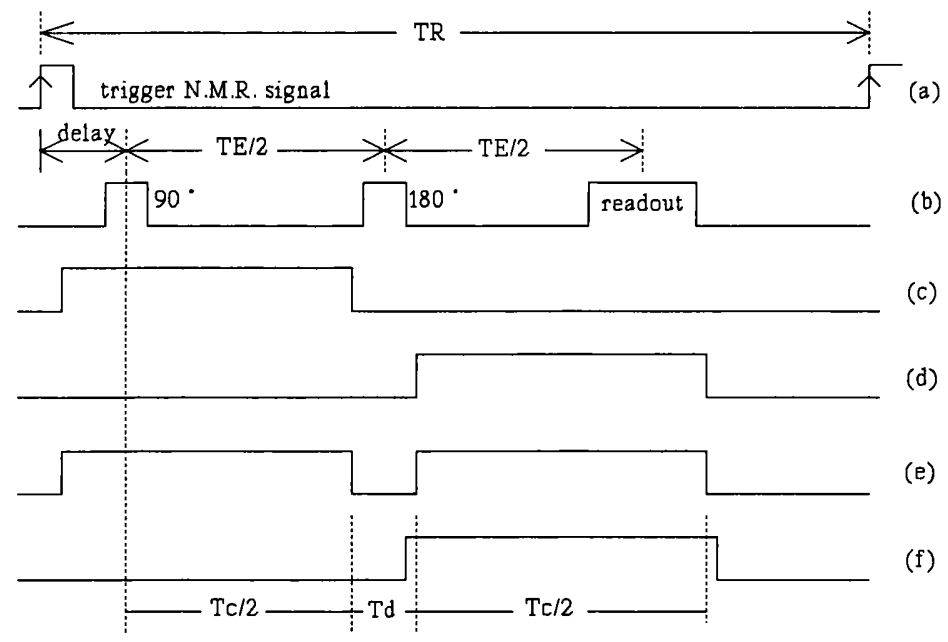

Figure C.4 - Signals to control the current pulses and NMR trigger. (a) NMR trigger, (b) NMR $90^{\circ}, 180^{\circ}$ and readout gradient pulses, $(c)(d)(e)$ and $(f)$ signals to control the current supply.

The Terminal Count Toggled output from counters 1 and 2 (figure C.4c and d) are passed through a logic OR, to produce one signal with two pulses (figure C.4, e). Each pulse has an on period equal to half the current time, and the time between the pulses is equal to the interval (TD) for the $180^{\circ} \mathrm{NMR}$ pulse. This signal and the other used to control the current direction are applied to two light emitters to send the signal to the current unit via a pair of optical fibres.

The clock signal for all the counters comes from one crystal oscillating at $1 \mathrm{MHz}$, which can be divided down as desired. Frequencies of 1 and $10 \mathrm{KHz}$ are used for the repetition time and current time pulse, respectively. 
The connection between this unit and the Current Source Unit, is made by a pair of optical fibres. This feature, avoids the entry of any interference from of the external environment into the NMR shielded cage. The optical fibres pass through a single small hole in the Faraday screen cage. 


\section{References}

[1] Abragam, A. (1961), The principles of nuclear magnetism.

[2] Barber, D. C. and Brown, B. H.(1984), "Applied potential tomography", Journal of Physics E, Scientific Instrumentations, Vol. 17, pp:723-733.

[3] Barber, D. C. (1993), "An overview of image reconstruction", in Clinical and physiological applications of Electrical Impedance Tomography, Edited by David Holder, Chapter Four, pp.47-60.

[4] Barber, D.C. and Brown, B. H. (1983), "Imaging Spatial Distributions of Resistivity Using Applied Potential Tomography", Electronics Letters, Vol. 19, No. $22,27^{\text {th }}$ October 1983 , pp. 933-935.

[5] Barber. D. C. and Seagar, A. D. (1987), "Fast reconstruction of resistance images", Clinical Physics and Physiological Measurements, Vol. 8, Suppl. A, pp:47-54.

[6] Baker, L. E. and Geddes, L. A. (1970), "The measurement of current density distribution in biological materials", Proceedings of the second international symposium Electrotherapy Sleep, Electroanesth., Graz, Austria, Vol. 11, pp. 3-11.

[7] Bayford, R.H.; Boone, K.G.; Hanquan, Y. and Holder, D. S. (in press), "Improvement of the positional accuracy of EIT images of the head using a lagrange multiplier reconstruction algorithm with diametric excitation", Clinical and Physiological Measurements, in press.

[8] Beall, P.T.; Amtey, S. R. and Kasturi, S. R., (1984), NMR Data Handbook for Biomedical Applications, Pergamon Press.

[9] Bonne K. G. (1995), "The possible use of applied potential tomography for imaging action potentials in the brain", $\mathrm{PhD}$ thesis, University of London.

[10] Boone, K., Lewis, A. M. and Holder, D. S. (1994), "Imaging of cortical spreading depression by EIT: implications for localization of epileptic foci", Physiological Measurement, Vol. 15, pp:A189-A198.

[11] Bottomley, P. A. and Andrew, E. R. (1978), "RF Magnetic Field Penetration, Phase Shift and Power Dissipation in Biological Tissue: Implications for NMR Imaging", Physics in Medicine and Biology, Vol. 23, No. 4, pp: 630-643.

[12] Brigham, E. O. (1974), "The Fast Fourier Transform", Prentice-Hall.

[13] Burger, H. C. and van Dongen, R. (1961), "Specific Electric Resistance of Body Tissues", Physics in Medicine and Biology, Vol. 5, pp:431-437. 
[14] Carlson, A. B. (1975), Communication Systems, McGraw-Hill.

[15] Choh-Luh Li, Anthony F. Bak and Levon O. Parker (1968), "Specific Resistivity of the Cerebral Cortex and White Matter", Experimental Neurology, Vol. 20, pp: 544-557.

[16] Clifford, A. A. (1973), Multivariate Error Analysis, Applied Science Publishers.

[17] Conturo, T. E. and Smith, G. D. (1990), "Signal-to-Noise in Phase Reconstruction: Dynamic Range Extension Using Phase Reference Offsets", Magnetic Resonance in Medicine, Vol. 15, pp:420-437.

[18] Cui, W; Ostrander, L. E. and Lee, B. Y., "In vivo Reflectance of Blood and Tissue as a Function of Light Wavelength", IEEE Transactions on Biomedical Engineering, Vol. 37, No. 6, pp: 632-639.

[19] Duck, F. A. (1990), Physical Properties of Tissue A Comprehensive Reference Book, Academic Press.

[20] Edelstein, W. A.; Glover, G. H.; Hardy, C. J. and Redington, R. W. (1986), "The Intrinsic Signal-to-Noise Ratio in NMR Imaging", Magnetic Resonance in Medicine, Vol. 3, pp: 604-618.

[21] Eisberg, R. M. (1985), Quantum physics of atoms molecules, solids, nuclei and particles, Wiley.

[22] Elwell, C. E. (1995a), A practical users guide to near infrared spectroscopy, Hamamatsu Photonics.

[23] Elwell, C. E. (1995b), "Measurement and Data Analysis Techniques for the Investigation of Adult Cerebral Haemodynamics using Near Infrared Spectroscopy", PhD thesis, University of London.

[24] Ernest, R. R. (1969), "Numerical Hilbert Transform and Automatic Phase Correction in Magnetic Resonance Spectroscopy", Journal of Magnetic Resonance, Vol. 1, pp:7-26.

[25] Geddes, L.A. and Baker, L. E.(1967), "The specific resistance of biological material - a compendium of data for the bio-medical engineer and physiologist", Medical and Biological Engineering, Vol. 5, pp:271-293.

[26] Geddes, L. A. and Baker, L. E. (1989), Principles of Applied Biomedical Instrumentation, Wiley.

[27] Ghiglia, D. C., Mastin, G. A. and Romero, L. A. (1987), "Cellular-automata method for phase unwrapping", Journal optical Society of America, Vol. 4, No. 1, pp. 267-280, January. 
[28] Gudbjartsson, H. and Patz, S. (1995), "The Rician Distribution of Noisy MRI Data", Magnetic Resonance in Medicine, Vol. 34, pp:910-914.

[29] Grollman, S. (1969), The Human Body Its Structure and Physiology, "2nd Edition, Macmillan.

[30] Hayt, Jr. W. H. (1989), Engineering Electromagnetics, McGraw-Hill.

[31] Hedley, M. \& Rosenfeld, D. (1992), "A New Two-Dimensional Phase Unwrapping Algorithm for MRI Images", Magnetic Resonance in Medicine, Vol. 24, pp. 177-181.

[32] Henkelman, R. M. (1985), "Measurement of signal intensities in the presence of noise in MR images", Medical Physics, Vol. 12, pp:232-233.

[33] Henkelman, R. M. and Bronskill, M. J. (1987), "Artifacts in magnetic resonance imaging", Review on Magnetic Resonance Imaging, vol. 2, no. 1, pp. 1-126.

[34] Holder, D. S. (1993b), "Physiological constraints to imaging brain function with EIT and scalp electrodes" in Clinical and physiological applications of Electrical Impedance Tomography, Edited by David Holder, Chapter 21, pp:185-200.

[35] Holder, D. S. and Brown, B. H. (1993), "Biomedical applications of EIT: a critical review", in Clinical and physiological applications of Electrical Impedance Tomography, Edited by David Holder, Chapter Two, pp:6-40.

[36] Holder, D. S.(1992), "Electrical impedance tomography with cortical or scalp electrodes during global cerebral ischaemia in the anaesthetised rat", Clinical Physics and Physiological Measurements, Vol 13, No. 1, pp:87-98.

[37] Holder, D. S. (1993a), "Opportunities for EIT in the nervous system", in Clinical and physiological applications of Electrical Impedance Tomography, Edited by David Holder, Chapter 19, pp.166-175.

[38] Holder, D. S. and Khan A. (1994), "Use of polyacrylamide gels in a saline-filled tank to determine the linearity of the Sheffield Mark 1 electrical impedance tomography (EIT) system in measuring impedance disturbances", Physiological Measurements, Vol. 15, pp:A45-A50.

[39] Holder, D. S.; Hanquan, Y. and Rao, A. (in press), "Some practical biological phantoms for calibrating multifrequency Electrical Impedance Tomography, Physiological Measurements.

[40] Hoult, D. I. and Lauterbur, P. C. (1979), "The Sensitivity of the Zeugmatographic Experimental Involving Human Samples", Journal of Magnetic Resonance, Vol. 34, pp: 425-433. 
[41] Hoult, D. I. and Richards, R. E. (1976), "The Signal-To-Noise of the Nuclear Magnetic Resonance Experiment", Journal of Magnetic Resonance, Vol. 24, pp: 71-85.

[42] Ider, Y. Z.; Muftuler, L. T. and Birgul O. (1995), "Use of MRI for measuring AC internal currents of EIT: A feasibility study", Proceedings of the IX International Conference on Electrical Bio-Impedance, Heidelberg-Germany, September 26-30, pp:420-421.

[43] Itoh, Kazuyoshi (1982), "Analysis of the phase unwrapping algorithm", Applied Optics, Vol. 21, No. 14, pp. 2470, 15 July.

[44] Joy, M.; Scott, G. and Henkelman, M. (1989), "In Vivo Detection of Applied Electric Currents By Magnetic Resonance Imaging", Magnetic Resonance Imaging, vol. 7, pp. 89-94.

[45] Keller, A. and Haeberlen, U. H. (1988), "Generalization of the Redfield-Kunz Treatment of Quadrature Phase Time Data", Hewlett-Packard Journal, December, pp: 74-76.

[46] Kotre, C. J.(1989), "A sensitivity coefficient method for the reconstruction of electrical impedance tomograms", Clinical Physics and Physiological Measurements, Vol. 10, No 3, pp:275-281.

[47] Libove, J. M. and Singer, J. R. (1980), "Resolution and signal-to-noise relationships in NMR imaging in the human body", Journal of Physics E: Science Instrumentation, Vol. 13, pp:38-44.

[48] Lin, Q., Vesecky, J. F. and Zebker, H. A. (1992), "Phase unwrapping through least squares approximation", SPIE Vol. 1657 Image Processing Algorithms and Techniques III, pp:244-255.

[49] Linderkamp, O.; Berg, D.; Betke, K. Koferl, F.; Kriegel, H. and Riegel, K. P. (1980), "Blood Volume and Hematocrit in Various Organs in Newborn Piglets", Pediatric Research, Vol. 14, pp:1324-1327.

[50] Ljunggren, S. (1983), "A Simple Graphical Representation of Fourier-Based Imaging Methods", Journal of Magnetic Resonance, Vol. 54, pp:338-343.

[51] Lytle, R. J.; Duba, A. G. and Willows, J. L. (1979), "Alternative methods for determining the electrical conductivity of core samples", Review of Science Instrumentation, Vol. 50, No. 5, May, pp:611-615.

[52] Marshall, A. G. and Verdun, F. R. (1990), Fourier Transforms in NMR, optical, and mass spectrometry - A User's Handbook, Elsevier Science Publishers B. V.

[53] McArdle, F. J.; Brown, B. H. and Angel, A. (1993), "Imaging cardiosynchronous 
impedance changes in the adult head", In Clinical and physiological applications of Electrical Impedance Tomography, Edited by David Holder, Chapter 20, pp:177-183.

[54] McVeigh, E. R., Henkelman, R. M. and Bronskill, M. J. (1985), "Noise and filtration in magnetic resonance imaging", Medical Physics, Vol. 12(5), pp:586591.

[55] Millman, J. and Halkias, C. C.(1972), Integrated Electronics: Analog and Digital Circuits and Systems, McGraw-Hill.

[56] Morris, P. G. (1986), Nuclear Magnetic Imaging in Medicine and Biology, Oxford University Press.

[57] Murphy et al. (1987), "Developments of the electrical impedance method with application to plethysmography and tomography", $\mathrm{PhD}$ thesis, University of Oxford in Gadd et al.(1993), "The Keele approach to EIT in the neonatal head", Chapter 22, Clinical and physiological applications of Electrical Impedance Tomography Edited by David Holder, 1993.

[58] Patel, M. and Hu, X. (1993), "Direct Calculation of Wrap-free Phase Image", Proceedings of the Society of Magnetic Resonance in Medicine, Vol. 2, Twelfth Annual Scientific Meeting, New York, NY, USA.

[59] Pethig, R. (1987), "Dielectric properties of body tissues", Clinical Physics and Physiological Measurements, Vol. 8, Suppl. A, pp:5-12.

[60] Plonsey, R. and Barr, R. (1982), "The Four-Electrode Resistivity Technique as Applied to Cardiac Muscle", IEEE Transactions on Biomedical Engineering, Vol. BME-29, No. 7, pp:541-546.

[61] Porter R.; Adey, W. R. and Kado, R. T. (1964), "Measurement of electrical impedance in the human brain", Neurol., Vol. 14, pp:1002-1012.

[62] Press, W. H.; Teukolsky, S. A.; Vetterling, W. T. and Flannery, B. P. (1992), Numerical Recipes in C, Cambridge University Press.

[63] Price, L. R. (1979), "Electrical Impedance Computed Tomography (ICT): A New CT Imaging Technique", IEEE Transactions on Nuclear Science, Vol. NS-26, No. 2, April, pp:2736-2739.

[64] Rack, J. B. (1963), "Analyses of Specific Impedance of Rabbit Cerebral Cortex", Experimental Neurology, Vol 7, pp:153-174.

[65] Ramo, S. Whinnery, J. R. and van Duzer, T. (1994), Field and waves in communication electronics, Wiley. 
[66] Redfield, A. G. and Kunz, Sara D. (1975), "Quadrature Fourier NMR Detection: Simple Multiplex for Dual Detection and Discussion", Journal of Magnetic Resonance, Vol. 19, pp: 250-254.

[67] Rinck, P. A. (1993), Magnetic Resonance in Medicine, Blackwell.

[68] Rolfe, P; Murdoch, N.; Tarassenko, L. and Weindling, A. M.(1982), "Can Electrical Impedance Tomography be used to study intraventricular haemorrhage?", The Second Special Ross Laboratories Conference on Perinatal Intracranial Haemorrhage, December 2-4, 1982, Vol 1.

[69] Rolfe, P.; Murdoch, N.; Tarassenko, L. and Weindling, A. M. (1982), "Can Electrical Impedance Plesthymography be used to study Infraventricular Haemorrhage ?", The Second Special Ross Laboratories Conference on Perinatal Intracranial Haemorrhage, Vol. 1, December 2-4, pp:380-399.

[70] Rush, S.; Abildskov, J. A. and McFee, R. (1963), "Resistivity of Body Tissues at Low Frequencies", Circulation Research, Vol. XII, January, pp:40-50.

[71] Sack, W.O. (1982), Essentials of pig anatomy.

[72] Sakai, F.; Nakazawa, K.; Tazaki Y., Katsumi, I., Hino, H., Igarashi, H. and Kanda, T. (1985), "Regional cerebral blood volume and haematocrit measurement in normal human volunteers by single emission computed tomography", J. of Cereb. Blood Flow \& Metab, Vol.5, pp:207-213.

[73] Scott, G. C.; Joy, M. L. G.; Armstrong, R. L. and Henkelman, R. M. (1995), "Rotating Frame RF Current Density Imaging", Magnetic Resonance in Medicine, Vol. 33, pp:355-369.

[74] Scott, G. C.; Joy, M. L. G.; Armstrong, R. L. and Helkelman, R. M. (1991), "Measurement of Nonuniform Current Density by Magnetic Resonance", IEEE Transactions on Medical Imaging, Vol. 10, No. 3, pp. 362-374.

[75] Scott, G. C.; Joy, M. L. G.; Armstrong, R. L. and Henkelman, R. M. (1992b), "Sensitivity of Magnetic-Resonance Current-Density Imaging", Journal of Magnetic Resonance, Vol.97, pp. 235-254.

[76] Scott, G. C.; Joy, M. L. G.; Armstrong, R. L. and Helkelman, R. L. (1992), "RF Current Density Imaging in Homogeneous Media", Magnetic Resonance in Medicine, Vol. 28, pp:186-201.

[77] Spik, A. (1991), "Investigation of the Cellular Automata Method for Phase Unwrapping and its Implementation on an Array Processor", Optics and Laser in Engineering, Vol. 14, pp:25-37.

[78] Thomas, D. C.; Siddall-Allum, I.A. Sutherland and Beard, R. W. (1993), "The 
use of EIT to assess blood volume changes in the female pelvis", in Clinical and physiological applications of Electrical Impedance Tomography Holder (1993), Chapter 25, pp:227-233.

[79] Thompson, J. D. M.; Joy, M. L. G. and Henkelman, R. M. (1991), "Current Density Imaging in Rabbit Head and Chest", Society of Magnetic Resonance in $M<e d i c i n e$, Tenth Annual Scientific Meeting and Exhibition, August 10-16, pp: 1274.

[80] Turner, C. J. and Hill, H. D. W. (1986), "Artifacts in Quadrature Detection", Journal of Magnetic Resonance, Vol. 66, pp: 410-421.

[81] van Harreveld, A. and Ochus, S. (1956), "Cerebral Impedance Changes After Circulatory Arrest", American Journal of Physiology, Vol. 187, pp:180-192.

[82] van Vaals, J.J. and van Gerwen, P.H.J. (1990), "Novel Methods for Automatic Phase Correction on NMR Spectra", Journal of Magnetic Resonance, Vol. 86, pp: $127-147$.

[83] Venot, A.; Lebruchec, J. F.; and Roucayrol, J. C. (1984), "A New Class of Similarity Measures for Robust Image Registration", Computer Vision, Graphics and Image Processing, Vol 28, pp. 176-184.

[84] Webb, S. (1995), The Physics of Medical Imaging.

[85] Webster, J. G.(1990), Electrical Impedance Tomography, Adam Hilger.

[86] Williams, L.R. and Leggett, R.W. (1989), "Reference values for resting blood flow to organs of man", Clinical Physiological Measurement, Vol. 10, No. 3, pp: 187-217.

[87] Witsoe, D. A. and Kinnen, E. (1967), "Electrical resistivity of Lung at $100 \mathrm{kHz}$,, Medical and Biological Engineering, Vol. 5, pp:239-248.

[88] Yip, G.; Joy, M. L. G.; Scott, G. C. and Henkelman, R. M. (1992), "In vivo Current Density Imaging" Proceedings of the Society of Magnetic Resonance in Medicine, pp:3917.

[89] Zhao, S.; Dodd, N.J.F.; Kaczynski, J.; Hawnaur, J.M. and Isherwood, I. (1993), "In vivo MR Imaging of Electrical Current Density Distribution in Mouse Tumour", Proceedings of the Society of Magnetic Resonance in Medicine, Vol. 3, 12th Anual Scientific Meeting, pp:1368. 